\title{
Liminality as a lens on social meaning: A cross-variable study of gender in New Zealand English
}

\author{
Evan Hazenberg
}

\author{
A thesis \\ submitted to Victoria University of Wellington \\ in fulfilment of the requirements for the degree of \\ Doctor of Philosophy
}





\section{Abstract}

The variationist sociolinguistic enterprise has been successful in developing models of structural (i.e., language-internal) drivers of variation and change, but one of the barriers preventing the development of a parallel model accounting for social drivers is the difficulty in operationalising salience. Using a corpus of sociolinguistic interviews collected in Auckland, New Zealand between 2013 and 2015, this project examines the relationship between language variation and gendered identity, and proposes an analytical approach of liminality - of examining the linguistic practices of people who have crossed a culturally-reified category boundary - as a possible solution to the problem of identifying socially salient variables.

The participants in this study are straight, queer, and transsexual native speakers of New Zealand English (NZE), representing two age groups that straddle the period of social change in the 1980s that saw a destabilisation of traditional gender roles in New Zealand. Variation in three linguistic systems is examined: adjectival modifiers (intensifiers and moderators), sibilants $(/ \mathrm{s} /, / \mathrm{z} /$ and $/ \mathrm{f} /$ ), and the vowels of NZE. The project uses established variationist and sociophonetic methodologies, as well as introducing a new metric for making comparisons across the vowel space as a whole. The findings show that speakers are able to encode their gendered identity across multiple variables, and that subtle linguistic signals can be used to affiliate with (or distance from) particular groups. The two most 'distant' groups are older straight men and younger [straight, queer, and transsexual] women, which this thesis argues is a reflection of the general societal criticism directed at young women by hegemonic masculinities. There is also evidence that the mainstreaming of queer identities in the wake of the 1986 decriminalisation of homosexuality has decreased the linguistic distance between straight and queer urban New Zealanders, demonstrating the relative rapidity with which social changes can be incorporated into the linguistic system.

This study highlights the utility of studying variation across linguistic systems rather than in variables in isolation, and proposes a typology of variation based on the boundedness and dimensionality of the linguistic systems under investigation. The framework of liminality is found to be productive with respect to the role of gender in language variation, although future research will have to test whether this framework can be generalised to other dimensions of social identity that are perceived as categorical (e.g., ethnicity and heritage culture in immigrant communities). 
As much as it may feel like a long and lonely road, a thesis is not a solitary endeavour; I am particularly indebted to the following people for their assistance and support over the past four years.

Miriam Meyerhoff and Sasha Calhoun

two truly wonderful supervisors

Janet Holmes, Meredith Marra, Janet Attrill and Bernie Hambleton

departmental support above and beyond

Richard Arnold

sage advice on statistics

Education New Zealand

generous financial support in the form of the New Zealand International

Doctoral Research Scholarship (NZIDRS)

406ers past and present

sanity and happy distractions as needed

family and friends

good humour, patience, and a willingness to listen to me go on about

linguistics long after they'd stopped caring

Erez Levon, Kevin Watson, Janet Holmes

thorough and thoughtful examiners

and most importantly,

the participants who have supported my research

for their time, commitment and generosity of spirit

\section{thank you}




\section{Table of Contents}

Abstract

Acknowledgements

List of Tables $\quad$ xiii

List of Figures $\quad$ xv

Some useful terms xvii

\section{Chapter one: Motivation}

1.1 Introduction 1

1.1.1 So what is this thesis?

1.2 Some generalisations from sociolinguistics 3

1.2.1 Labov's principles of change 4

1.2.1.1 Principle I 4

1.2.1.2 Principle Ia 4

1.2.1.3 Principle II 4

1.2.1.4 Gender paradox?

1.2.2 Salience 6

1.2.2.1 Indicators, markers, stereotypes 6

$\begin{array}{lll}\text { 1.2.2.2 } & \text { Salience as gradient } & 7\end{array}$

1.2.2.3 Salience in this project 8

1.2.3 Eckert's three waves of variationist studies $\quad 8$

1.2.3.1 Contextualising the third wave 9

$\begin{array}{ll}1.3 \text { Making meaning } & 11\end{array}$

1.3.1 Social constructionism and critical realism 11

$\begin{array}{ll}\text { 1.3.2 Prototypes and stereotypes } & 13\end{array}$

$\begin{array}{lll}\text { 1.3.3 Strategic essentialism } & 14\end{array}$

1.4 Bridging the linguistic and the social 15

$\begin{array}{ll}\text { 1.4.1 Enregisterment } & 16\end{array}$

1.4.1.1 Asymmetries of competence and register complexity 16

1.4.1.2 Awareness and evaluation of difference 17

1.4.1.3 Evaluations are disseminated and embraced $\quad 18$

$\begin{array}{ll}1.4 .2 & \text { Indexicality } \\ 1.4 .3 & 19\end{array}$

1.4.3 Gender as performance 22

1.4.4 Trans identities as liminal 22

1.5 What this thesis aims to do 24 


\section{Chapter two: Community}

2.1 Introduction $\quad 27$

2.2 Social change(s) in New Zealand 28

2.2.1 Changing gender roles: masculinity 28

2.2.2 Changing gender roles: femininity 30

2.2.3 The Homosexual Law Reform bill 31

2.3 The Auckland Trans Corpus (ATC) 33

2.3.1 Designing the corpus 34

$\begin{array}{lll}2.3 .2 & \text { ATC participants } & 37\end{array}$

2.3.2.1 Straight participants 37

2.3.2.2 Queer participants $\quad 39$

2.3.2.3 Trans participants 41

2.3.3 The ATC as a linguistic resource $\quad 44$

\section{Chapter three: Methods}

3.1 Introduction $\quad 47$

$\begin{array}{lll}3.2 & \text { Data collection } & 47\end{array}$

3.2.1 The sociolinguistic interview 48

3.2.2 My role as interviewer 49

$\begin{array}{lll}3.2 .3 & \text { The structure of the interview } & 50\end{array}$

3.3 Identifying and working with extracts 51

3.3.1 Selecting extracts $\quad 52$

3.3.2 Transcription 53

3.4 Corpus linguistics tools: looking for adjectives 53

3.5 Phonetic analysis tools $\quad 54$

3.5.1 Automatic alignment tools 55

3.5.1.1 FAVE-extract 56

3.5.1.2 Shortcomings of FAVE $\quad 57$

3.5.2 Semi-automatic tools for sibilant analysis 58

$\begin{array}{lll}3.5 .2 .1 & \text { Praat } & 58\end{array}$

3.6 Analytical approaches 59

3.6.1 Descriptive statistics $\quad 59$

3.6.1.1 ANOVA 59

3.6.1.2 Tukey's HSD and homogeneous subsets 60

$\begin{array}{lll}3.6 .2 & \text { Multivariate analysis } & 60\end{array}$

3.7 Variable-specific particulars $\quad 62$

\section{Chapter four: Adjectival modifiers}

4.1 Introduction 63

$\begin{array}{ll}\text { 4.1.1 What are intensifiers? } & 63\end{array}$ 
4.1.2 Intensifiers in NZE $\quad 65$

4.1.3 Language change 66

4.1.4 Social difference $\quad 66$

4.1.5 A note about downtoners and moderators 68

$\begin{array}{lll}4.2 & \text { Methods } & 68\end{array}$

4.2.1 Finding adjectives in the interviews 68

$\begin{array}{lll}\text { 4.2.2 Identifying appropriate adjective heads } & 69\end{array}$

$\begin{array}{ll}\text { 4.2.3 Coding adjectives for analysis } & 70\end{array}$

4.2.3.1 Type of modification $\quad 71$

$\begin{array}{lll}4.2 .3 .2 & \text { Sentence polarity } & 71\end{array}$

$\begin{array}{lll}\text { 4.2.3.3 Syntactic position } & 73\end{array}$

4.2.3.4 Type of adjective $\quad 73$

4.2.3.5 Evaluative orientation of the adjective 74

$\begin{array}{ll}4.2 .4 \text { Analysis } & 74\end{array}$

4.3 Intensifiers $\quad 76$

$\begin{array}{lll}\text { 4.3.1 Overall adjectival modification } & 76\end{array}$

$\begin{array}{lll}\text { 4.3.2 Distributional results } & 78\end{array}$

$\begin{array}{lll}\text { 4.3.3 Statistical models of intensifier choice } & 80\end{array}$

$\begin{array}{lll}4.3 .3 .1 & \text { Really } & 80\end{array}$

4.3.3.2 Very 82

$\begin{array}{lll}4.3 .3 .3 \text { So } & 83\end{array}$

$\begin{array}{lll}4.4 & \text { Moderators } & 84\end{array}$

$\begin{array}{lll}\text { 4.4.1 Distribution } & 84\end{array}$

4.4.1.1 Kind of/sort of $\quad 85$

4.4.1.2 Quite 85

4.4.1.3 Litotes $\quad 86$

4.4.1.4 A (little) bit and enough $\quad 86$

4.5 Trans speakers $\quad 86$

$\begin{array}{lll}4.6 & \text { Discussion } & 89\end{array}$

4.6.1 Relative (structural) stability of the system 89

$\begin{array}{ll}\text { 4.6.2 Age as a perceptual lens } & 90\end{array}$

4.6.3 Intensifiers as a gendered resource 91

\section{Chapter five: Sibilants}

5.1 Introduction 93

$\begin{array}{lll}5.1 .1 & \text { Sibilants } & 95\end{array}$

5.1.2 What this chapter aims to do 96

5.2 Spectral moments 96

5.2.1 First moment: Centre of Gravity 97

5.2.2 Second moment: Standard Deviation 97

5.2.3 Third moment: Skewness 99 
5.2.4 Fourth moment: Kurtosis 99

5.2.5 Fricative moments and gender 99

5.2.6 What are CofG and Skewness actually measuring? 99

$\begin{array}{lll}5.3 \text { Why sibilants? } & 100\end{array}$

$\begin{array}{ll}\text { 5.3.1 Sibilants, sex, and gender } & 101\end{array}$

$\begin{array}{ll}5.3 .2[\mathrm{~s}] \text { and sexuality } & 102\end{array}$

$\begin{array}{ll}\text { 5.3.3 [s] and trans identities } & 103\end{array}$

$\begin{array}{ll}\text { 5.3.4 A quick note on voicing } & 104\end{array}$

$\begin{array}{lll}\text { 5.3.5 Summary of previous findings } & 105\end{array}$

5.4 Methods 106

5.4.1 Correcting for biological differences? 107

$\begin{array}{lll}5.4 .2 & \text { Tokens } & 107\end{array}$

$5.4 .2 .1 / \mathrm{s} /$ and $/ \mathrm{g} / \quad 108$

5.4.2.2 /s/ and /z/ 108

$\begin{array}{ll}\text { 5.4.3 Analytical approach } & 109\end{array}$

$\begin{array}{ll}\text { 5.4.4 A note about independence } & 109\end{array}$

$\begin{array}{ll}5.5 \text { /s/ } & 110\end{array}$

$\begin{array}{lll}5.5 .1 / \mathrm{s} /: \text { Distribution } & 110\end{array}$

$\begin{array}{lll}\text { 5.5.1.1 Means } & 112\end{array}$

5.5.1.2 Homogeneous subsets 113

$\begin{array}{ll}5.5 .2 / \mathrm{s} / \text { : Statistical modeling } & 115\end{array}$

$\begin{array}{ll}\text { 5.5.2.1 Linguistic constraints } & 117\end{array}$

$\begin{array}{ll}\text { 5.5.2.2 Social constraints } & 117\end{array}$

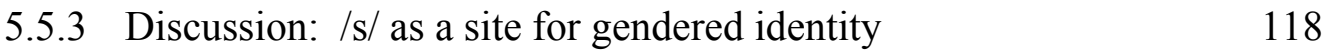

$\begin{array}{ll}5.6 \mathrm{lz} / & 121\end{array}$

$\begin{array}{lll}5.6 .1 / \mathrm{z} /: \text { : Distribution } & 123\end{array}$

5.6.1.1 Means 123

5.6.1.2 Homogeneous subsets 126

$\begin{array}{ll}5.6 .2 \mathrm{~K} / \mathrm{z} \text { : Statistical modeling } & 126\end{array}$

$\begin{array}{ll}\text { 5.6.2.1 Linguistic constraints } & 126\end{array}$

$\begin{array}{ll}\text { 5.6.2.2 Social constraints } & 128\end{array}$

5.6.3 Discussion: / $\mathrm{z} /$ as a site for gendered identity 129

$\begin{array}{lll}5.7 / \mathrm{J} / & 130\end{array}$

$\begin{array}{lll}5.7 .1 / S /: \text { Distribution } & 132\end{array}$

$\begin{array}{lll}\text { 5.7.1.1 Means } & 132\end{array}$

5.7.1.2 Homogeneous subsets 133

$\begin{array}{lll}5.7 .2 / \mathrm{J} / \text { : Statistical modeling } & 135\end{array}$

$\begin{array}{lll}\text { 5.7.3 Discussion: / } / \text { / as a site for gendered identity } & 136\end{array}$

5.8 General discussion of sibilants in NZE 136

$\begin{array}{ll}\text { 5.8.1 A model of social meaning for sibilants } & 138\end{array}$ 


\section{Chapter six: Vowels}

6.1 Introduction 141

$\begin{array}{ll}\text { 6.1.1 Roadmap for this chapter } & 142\end{array}$

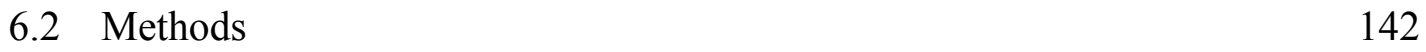

6.2.1 Automatic vowel measurement 142

$\begin{array}{ll}\text { 6.2.2 Output from FAVE-extract } & 143\end{array}$

$\begin{array}{lll}6.2 .3 & \text { Outliers } & 145\end{array}$

$\begin{array}{lll}6.2 .4 & \text { Vowel normalisation } & 146\end{array}$

$\begin{array}{ll}\text { 6.2.5 Total number of vowel tokens } & 149\end{array}$

6.2.6 What to compare: the Cartesian vowel plot 149

6.2.6.1 Euclidean Distance 150

6.2.6.2 Scale 152

6.2.6.3 A note on the use of Hertz 153

6.2.6.4 Analysis of gendered production 153

$\begin{array}{lll}6.3 & \text { Findings } & 155\end{array}$

$\begin{array}{lll}\text { 6.3.1 NZE vowel space generally } & 155\end{array}$

$\begin{array}{ll}\text { 6.3.2 A closer look at the vowel space } & 156\end{array}$

$\begin{array}{lll}\text { 6.3.2.1 Monophthongs } & 158\end{array}$

6.3.2.2 Diphthongs 159

6.3.2.3 Summary of the vowels for analysis 160

$\begin{array}{lll}\text { 6.3.3 Stable vowels } & 161\end{array}$

6.3.3.1 FLEECE 162

6.3.3.2 START 163

$\begin{array}{lll}6.3 .3 .3 & \text { GOOSE } & 164\end{array}$

6.3.3.4 LOT 165

$\begin{array}{lll}\text { 6.3.4 Vowels showing a clear trend } & 167\end{array}$

6.3.4.1 FACE and GOAT 167

6.3.4.1.1 FACE 167

6.3.4.1.2 GOAT 169

6.3.4.1.3 Some observations on FACE and GOAT 170

$\begin{array}{lll}\text { 6.3.4.2 DRESS and KIT } & 170\end{array}$

6.3.4.2.1 DRESS 171

6.3.4.2.2 KIT 173

6.3.4.2.3 Some observations on DRESS and KIT 173

$\begin{array}{lll}\text { 6.3.4.3 FOOT and NURSE } & 175\end{array}$

$\begin{array}{lll}6.3 .4 .3 .1 & \text { FOOT } & 175\end{array}$

$\begin{array}{lll}\text { 6.3.4.3.2 NURSE } & 177\end{array}$

6.3.4.3.3 Some observations on FOOT and NURSE 179

6.3.4.4 MOUTH and PRICE 181

6.3.4.4.1 MOUTH onset 181

6.3.4.4.2 MOUTH offglide 181 
6.3.4.4.3 PRICE onset 182

6.3.4.4.4 PRICE offglide 184

6.3.4.4.5 Some observations on MOUTH and PRICE 184

6.3.4.5 STRUT and TRAP 185

6.3.4.5.1 STRUT 185

6.3.4.5.2 TRAP 185

6.3.4.5.3 Some observations on STRUT and TRAP 188

$\begin{array}{ll}\text { 6.3.5 Mergers, near-mergers, and un-mergers } & 189\end{array}$

6.3.5.1 NEAR and SQUARE 189

6.3.5.1.1 NEAR and SQUARE in this data 191

6.3.5.2 NORTH and THOUGHT 192

6.3.5.2.1 NORTH and THOUGHT in this data 195

$\begin{array}{lll}\text { 6.3.5.3 START and STRUT } & 195\end{array}$

6.3.5.3.1 START and STRUT in this data 197

6.3.6 Comparing vowel spaces: the Index of Similarity 197

6.3.6.1 Computing pairwise comparisons 198

$\begin{array}{lll}6.3 .6 .2 & \text { Results } & 200\end{array}$

6.3.6.3 Ranking by IoS score 202

6.4 Discussion 203

6.4.1 General discussion of New Zealand English vowels 203

$\begin{array}{lll}\text { 6.4.2 Some patterns } & 207\end{array}$

6.4.3 Sources of differences between this and previous studies 208

\section{Chapter seven: Synthesis}

$\begin{array}{lll}7.1 & \text { Introduction } & 209\end{array}$

$\begin{array}{lll}7.2 & \text { Variation within a system } & 209\end{array}$

$\begin{array}{ll}\text { 7.2.1 Dimensionality and boundedness } & 209\end{array}$

$\begin{array}{ll}\text { 7.2.2 Contextualising variation } & 212\end{array}$

7.3 Three waves of variation revisited 213

7.3.1 Operationalising gendered identity in this project 214

7.3.2 Second-wave platform for supporting third-wave studies 215

7.4 Social realities reflected in linguistic practice 215

7.4.1 Divergence: older straight men and younger women 216

$\begin{array}{ll}\text { 7.4.2 Divergence: older straight and queer men } & 217\end{array}$

$\begin{array}{ll}\text { 7.4.3 Convergence: queer identities in vowel space } 218 & 217\end{array}$

$\begin{array}{lll}\text { 7.4.3.1 Queer men } \sim \text { queer women } & 218\end{array}$

7.4.3.2 Older queer men $\sim$ older straight women $\quad 219$

7.4.3.3 Younger queer women $\sim$ younger straight women $\quad 219$

7.4.4 Convergence: South Auckland and young masculinity 220

7.5 Multiple lenses of social perception 222

$\begin{array}{lll}\text { 7.5.1 Age as a perceptual lens } & 222\end{array}$ 
7.5.2 Gender as a perceptual lens

7.6 Liminality and social drivers of change

7.6.1 Going beyond perception of studies 226

7.6.2 Transsexuality as a window into gendered indexicality 227

7.6.3 Beyond transsexuality: liminal identities 228

7.6.4 Revisiting Labov's variable typology 229

\section{Chapter eight: Reflections}

8.1 Introduction 233

8.2 Review of findings 233

8.2.1 Research aim 1: Gendered identity and variation 233

8.2.2 Research aim 2: Social change reflected in linguistic practice 235

8.2.3 Research aim 3: Liminality as a new approach 236

8.3 Where to next? 238

$\begin{array}{lll}\text { 8.3.1 Triangulation } & 238\end{array}$

$\begin{array}{lll}8.3 .2 & \text { Liminality } 239\end{array}$

8.3.3 Variation in systems 241

8.3.4 Language and gender 242

8.4 Final thoughts 243

References 245

\section{Appendices}

A The International Phonetic Alphabet (IPA) 265

B Praat script for sibilant identification and measurement 267

C URLs for blogs discussing (str) retraction $\quad 269$

D Means for vowel measurements 271

E Participant information sheet and consent form 279 


\section{List of Tables}

2.1 Straight participants in the ATC 38

2.2 Queer participants in the ATC 39

2.3 Trans participants in the ATC 41

4.1 Total number of adjective heads for analysis $\quad 69$

4.2 Comparison of overall rates across studies $\quad 75$

4.3 Rates of modification in Auckland across age groups 75

4.4 Adjectival modification by gender group 75

4.5 Multivariate analysis of modified vs unmodified adjectives - social 77 factors only

4.6 Most common intensifiers (comprising at least $5 \%$ of the data) 78

4.7 Intensifier choice by gender group as percentage of modified total 79

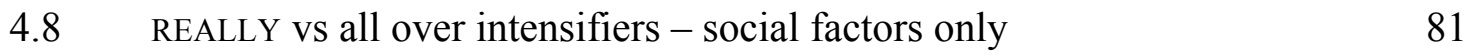

4.9 VERY vs all other intensifiers - social factors only 83

4.10 Most common moderators (comprising at least 5\% of the data) 84

4.11 Moderator choice by gender group as percentage of modified total 85

$\begin{array}{lll}4.12 & \text { Similarities in modifier choice for trans speakers } & 87\end{array}$

5.1 English sibilants 95

5.2 Summary of previous studies on /s/ and gendered identity 105

$\begin{array}{ll}5.3 \text { Total number of sibilant tokens } & 108\end{array}$

$5.4 \mathrm{~s} / \mathrm{CofG}$ - means and standard deviations $\quad 111$

$5.5 \mathrm{~s} / \mathrm{Skewness}$ - means and standard deviations $\quad 111$

$5.6 / \mathrm{s} / \mathrm{CofG}$ - homogeneous subsets (Tukey HSD) 114

$5.7 \quad / \mathrm{s} /$ Skewness - homogeneous subsets (Tukey HSD) 114

5.8 Multivariate analysis of /s/ CofG 116

5.9 Multivariate analysis of /s/ Skewness 116

$5.10 \mathrm{z} / \mathrm{z} / \mathrm{CofG}$ - means and standard deviations 122

$5.11 \mathrm{l} / \mathrm{z} /$ Skewness - means and standard deviations 122

$5.12 \mathrm{~s} / \mathrm{s}$ and $/ \mathrm{z} /$ CofG means and difference between means $(\mathrm{Hz}) \quad 124$

$5.13 \mathrm{lz} / \mathrm{CofG}$ - homogeneous subsets (Tukey HSD) 125

5.14 /z/ Skewness - homogeneous subsets (Tukey HSD) 125

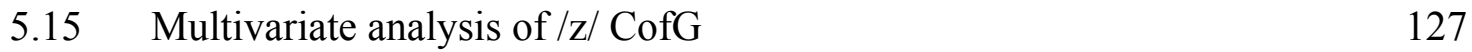

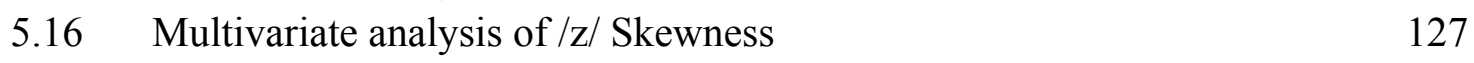

$5.17 \mathrm{~J} / \mathrm{CofG}$ - means and standard deviations 131

$5.18 / \mathrm{J} /$ Skewness - means and standard deviations 131

5.19 / $/$ / CofG - homogeneous subsets (Tukey HSD) 134

5.20 /S/ Skewness - homogeneous subsets (Tukey HSD) 134

5.21 Summary of $/ \mathrm{J} / \mathrm{CofG}$ multivariate analysis 135

$\begin{array}{ll}5.22 & \text { Summary of } / \mathrm{J} / \text { Skewness multivariate analysis } \\ & 135\end{array}$ 
5.23 Gendered effects and meanings across sibilants by place of articulation

6.1 Summary of vowel tokens by vowel and by speaker group 148

6.2 Summary of vowels for analysis 160

6.3 Multivariate analysis of FACE onset 166

6.4 Multivariate analysis of GOAT onset 168

6.5 Multivariate analysis of DRESS 172

$\begin{array}{lll}6.6 & \text { Multivariate analysis of KIT } & 174\end{array}$

$\begin{array}{lll}6.7 & \text { Multivariate analysis of FOOT } & 176\end{array}$

$\begin{array}{lll}6.8 & \text { Multivariate analysis of NURSE } & 178\end{array}$

6.9 Multivariate analysis of MOUTH onset 180

6.10 Summary of MOUTH offglides $\quad 182$

6.11 Multivariate analysis of PRICE onset 183

$\begin{array}{lll}6.12 & \text { Summary of PRICE offglides } & 184\end{array}$

6.13 Multivariate analysis of STRUT 186

$\begin{array}{lll}6.14 & \text { Multivariate analysis of TRAP } & 187\end{array}$

6.15 (Un)merger of NEAR and SQUARE 190

6.16 Separation of NORTH and THOUGHT 194

6.17 Separation of START and STRUT 196

6.18 Sample Index of Similarity counts (1) 198

6.19 Sample Index of Similarity counts (2) 199

6.20 Sample Index of Similarity counts $(1+2) \quad 199$

6.21 Index of Similarity for NZE vowel space 200

6.22 Ranking gendered groups by IoS 202

$\begin{array}{ll}6.23 & \text { Summary of vowel findings } \\ & 204\end{array}$ 


\section{List of Figures}

2.1 Network diagram of participants in the ATC 36

$\begin{array}{lll}4.1 & \text { Adjective in attributive position } & 72\end{array}$

$\begin{array}{lll}4.2 & \text { Adjective in predicative position } & 72\end{array}$

$\begin{array}{lll}4.3 & \text { NP-internal postpositive AP } & 72\end{array}$

$\begin{array}{lll}4.4 & \text { Postpositive AP as reduced relative clause } & 72\end{array}$

4.5 Adjectival modification by gender group (percentage) 77

5.1 Samples of fricatives from the speech of Caroline 93

5.2 The word so spoken by Illeana and Richard 94

5.3 First moment: Centre of Gravity (weighted mean) 97

5.4 Second moment: Standard Deviation 98

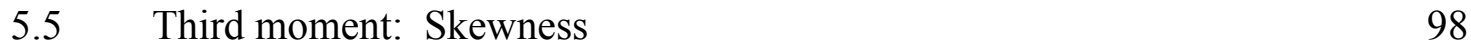

5.6 Fourth moment: Kurtosis 98

$\begin{array}{lll}5.7 & \text { Articulatory and phonetic correlates of } / \mathrm{s} / & 100\end{array}$

$\begin{array}{lll}5.8 / \mathrm{s} / \text { CofG vs. Skewness } & 112\end{array}$

5.9 Parallel continua for gendered indexicality of /s/ 119

5.10 Revised parallel continua for gendered indexicality of/s/ 120

5.11 /z/ CofG vs. Skewness 123

5.12 / $/$ / CofG vs. Skewness 132

5.13 Revised parallel continua for gendered indexicality of /s/ 138 (reproduced)

5.14 Schema for social interpretability of /s/ 140

6.1 Calculating Euclidean Distance (ED) 151

6.2 Conditions for Euclidean Distance measurement 151

6.3 Exaggerated effect of different scales on $\mathrm{x}$ and $\mathrm{y}$ axes 152

$\begin{array}{lll}6.4 & \text { Overall mean values for all vowels } & 155\end{array}$

6.5 Overview of vowel space of New Zealand English 157

6.6 Monophthongs of New Zealand English 158

6.7 Diphthongs of New Zealand English 159

6.8 Vowel plot for FLEECE 162

6.9 Vowel plot for START 163

$\begin{array}{lll}6.10 & \text { Vowel plot for GOOSE } & 164\end{array}$

6.11 Vowel plot for LOT 165

6.12 Vowel plot for FACE 166

6.13 Vowel plot for GOAT 168

$\begin{array}{lll}6.14 & \text { Vowel plot for DRESS } & 172\end{array}$

$\begin{array}{lll}6.15 & \text { Vowel plot for KIT } & 174\end{array}$

$\begin{array}{lll}6.16 & \text { Vowel plot for FOOT } & 176\end{array}$

$\begin{array}{lll}6.17 & \text { Vowel plot for NURSE } & 178\end{array}$ 
6.18 Vowel plot for MOUTH 180

6.19 Vowel plot for PRICE $\quad 183$

6.20 Vowel plot for STRUT 186

$\begin{array}{lll}6.21 & \text { Vowel plot for TRAP } & 187\end{array}$

6.22 Vowel plot for NEAR and SQUARE 190

6.23 Vowel plot for NORTH 193

6.24 Vowel plot for THOUGHT 193

6.25 Vowel plot for NORTH and THOUGHT together 194

6.26 Vowel plot for START and STRUT 196

7.1 Chain of indexical association linking younger trans women and 221 younger straight men

7.2 Revised schema of social interpretability for sibilants 223

7.3 Sample indexical associations of gendered /s/ 224 


\section{Some useful terms}

The definitions provided below draw on those published in New Zealand's Human Rights Commission report on discrimination experienced by transgender people (HRC 2008), but they also reflect the ways that my participants used these terms. Some of these words are problematic, as their definitions are malleable and under constant re-negotiation within the community, and it is impossible to include all the various shades of meaning that the words may encompass. Others are problematic because the terms themselves are contested; this is particularly true with terms that have, in the past, been used in a derogatory manner but have now become relatively neutral.

With these limitations in mind, the approach taken in developing this glossary has been to present a coherent account of the terminology as it is used in this thesis, and which reflects (to the extent that this is possible) how the terms are used by the queer and trans participants who have contributed to this research. Readers should be cautioned that these definitions will not necessarily align with those used elsewhere, such as other research projects, publications (both academic and otherwise), in the community, or in the media. These definitions should not be considered authoritative outside the bounds of this thesis.

Sex: In practice, people use the term sex to refer to the biological classification of people as either male or female or intersex, as determined by body morphology (internal and external genitalia, secondary sex characteristics) and chromosomes. Note that not everyone agrees that biology is either binary or deterministic; see $\S 1.3 .1$ for a brief overview of critiques to this deterministic perspective.

Gender: The socially-constructed matrix of identity that in Western cultures aligns largely with masculine (men) and feminine (women) expressions of identity. The normative association is that people classified as male identify as men and present masculine identities, and that those classified as female identify as women and present feminine identities.

Trans: When used on its own, trans is an umbrella term for people whose gender does not align with the sex they were assigned at birth; this encompasses transgender, transsexual, genderfluid, genderfuck, gender-non-conforming (GNC), and a range of other non-binary and/or misaligned identities. When used as a modifier of a gendered noun (e.g., trans woman, trans-masculine, etc.), the gendered noun indicates the identity of the individual (i.e., a trans man identifies and lives as a man, in spite of his previous experience in a sociallyfeminine role).

Transsexual: Transsexuals are trans people who have sought out some form of medical treatment (i.e., hormone therapy, surgical intervention), or who have adopted a 
gendered presentation without recourse to medical treatment, in order to present and live fully in the social role of their identified gender.

Transgender: Trans people who feel a disconnection between their identified gender and that assigned at birth, but who do not intend to pursue any medical interventions to bring their body into alignment with their internal sense of self, and who do not live their lives with a fixed gendered presentation (i.e., consistently presenting as men or women). This is a broader category than transsexual, and may include cross-dressers, genderfluid, and non-binary people.

Cissexual (cis): The opposite of transsexual: someone whose physical body and internal sense of identity align, and no intervention is required to live fully in their identified gender. Cis can also be used as a modifier, in the same way that trans can (i.e., cis woman = cissexual woman).

Sexuality: A personal characteristic that indexes who someone is emotionally, romantically, and/or sexually attracted to. Common sexualities include heterosexual (attracted to the people of the opposite gender, often called straight), homosexual (attracted to people of the same gender, often called gay or queer), and bisexual (attracted to people of both genders). There are other sexualities that fall into and out of usage, such as pansexual (attracted to people regardless of gender, inclusive of non-binary genders), asexual (not attracted to people of any gender), and polysexual (attracted to multiple partners and polyamorous relationships). Sometimes called sexual orientation.

Queer: An umbrella term used to refer to anyone who falls outside of a heteronormative identity, or to the communities and organisations that such people belong to. The history of this term is particularly contentious as it has been used as a slur against gay men and lesbians, but its use as a reclaimed word has been increasing with the acknowledgement that the commonly used terms gay, lesbian, and bisexual further marginalise people with other identities. Acronyms can be used (LGBT = Lesbian, Gay, Bisexual, Transgender), but these are equally exclusive, and attempts to make them more inclusive result in untenably long forms (e.g., LGBTTQQIA2FF*), making queer a default inclusive term in the community.

Straight: When used to describe an individual, heterosexual. When used to describe a set of cultural assumptions or institutions, heteronormative.

Gendered identity: A composite term that indexes gender, sexuality, and identity (i.e., queer cis woman, straight trans man). It recognises that these three dimensions of gendered experience and identity are independent but related, and often cooccur in culturally and socially meaningful clusters. 


\section{Chapter one: Motivation}

\subsection{Introduction}

The intersection of language and gender has always fascinated me. Even before I had a sense of either language or gender as things that were studiable, asymmetries bothered me: why were boys allowed to yell and swear on the playground, but not girls? Why did girls get into more trouble than boys for not saying 'please' and 'thank you'? Why could boys ask for a blue crayon, or even a light blue one, but never sky blue or (heaven forbid) periwinkle? At a fundamental level, of course, these childhood observations were about gender more than they were about language, but I was still paying attention to the differences in acceptable ways of speaking. Language struck me as a tool to communicate, but it was also a tool to control, and to limit, the kinds of things we were permitted to communicate.

This fascination stayed with me through my high school immersion in queer and artsy circles, and into university where, as a volunteer and later a staff member at the university's LGBTQ resource centre, I watched a succession of young men wander through the door in September and try to figure out how to be gay. By November many of them had gone full camp, with hair and clothes and mannerisms and, most important from my point of view, "the voice": that hard-to-pin-down cluster of linguistic features that is immediately recognisable as gay male (e.g., Zwicky 1997; Cameron \& Kulick 2003). By February a lot of them had toned it down, and found some kind of happy medium they could live with, some authentic way of maintaining their own sense of self in a sea of normative pressures to be just as gay as everyone else. Oddly there didn't seem to be the same kind of stylistic or linguistic negotiation going on with the women who came to the centre, and although there did seem to be a broad consensus that some women sounded more like lesbians than others, those conversations were never as hotly debated as they were between the men. This also struck me as interesting: why was it so much more important for men than for women?

And then there was the only marginally more sophisticated game of Gay or European that we played with visiting scholars in my third year linguistics courses. It was an exercise in teasing apart the overlapping social indices of European metrosexuality and North American queerness, but it did force us to frame the question from a linguistic perspective: which features were we paying attention to, and how were we interpreting them? Why did some forms signal gay for one speaker, but European for someone else? Ultimately we decided that it wasn't down to one or two features on their own, but rather to particular bundles of [+European] and [+gay] that seemed to average out in some way.

By this point in my career as a linguist, I was discovering more and more 
sophisticated approaches to asking these kinds of questions, and coming to the conclusion that they couldn't meaningfully be asked in isolation. They were intimately tied up in culture and context, to say nothing of the structural factors of language as a system: a good analysis of language and gender, then, had to be both sociolinguistic (paying attention to macro and micro social effects) and sociolinguistic (paying attention to the systems and semantics of communication). The variationist enterprise seemed to fit the bill, with its analytical focus on both the structural and the social constraints of language variation; it presented a reliable tool for teasing apart multiple sources of variation, and of focusing on particular components of the system without ignoring the other forces acting to shape the variation.

\subsubsection{So what is this thesis?}

This thesis is an exploration of gendered identity in New Zealand English. It pushes through the simple binary that a strictly biological perspective on gender would seem to favour, and adopts a more socially-constructed, multi-dimensional orientation that encompasses feminine/masculine, sexuality, and age. It draws on variationist sociolinguistic and sociophonetic methodologies to look at three systems of variation, and maps the variation in linguistic practice to the social dynamics of the speech community.

Within this framework, the thesis also considers a potential solution to the problem of disentangling social drivers of language variation from the social effects of structural change. The variationist programme in sociolinguistics has developed models of language change that make use of gender as a diagnostic of change in progress, with particular patterns expected for different types of variables ( $\$ 1.2 .1$ below). Some variables are transparently interpretable to members of the speech community and may therefore behave in one way, while others that are opaque may be markedly different in their distribution and systematicity. These regularities are useful in interpreting observed variation and change, but they can also complicate interpretation of social meaning. If we observe that young women are using a particular variant at significantly higher rates than young men, how do we decide if that variable is indexing gender, or if the gender pattern is simply a product of the type of structural change underway with that variable? Researchers have tended to look to perception studies for evidence of social meaning, and there are doubtless advantages to this approach, but it would be nice to be able to make inferences based on the production data that variationists have already collected and analysed. This thesis explores the idea of liminality - of crossing category boundaries as a tool to investigate the meaning-making interpretability of variables.

Because this study draws on methodologies and frameworks from several disciplines within the field of linguistics, this chapter will provide a brief introduction to the key concepts that are foundational to the project. We first look at some of the core generalisations that we will draw on from sociolinguistics, and then some of the relevant ideas from ontology-epistemology addressing how we make and interpret meaning in the 
world around us. Finally, we will lay out how to bridge these two approaches, before presenting a roadmap for the thesis as a whole.

\subsection{Some generalisations from sociolinguistics}

Since its inception in the 1960s, the variationist paradigm in sociolinguistics has been a particularly good tool for exploring the relationship between social factors and linguistic variability. The earliest studies (e.g., Labov 1966, 1972; Wolfram 1969; Trudgill 1974) found linguistic patterns associated with age, sex, ethnicity, and social class, and argued that how a person uses language is influenced by how they fit into the social fabric of their communities. Since then, variationist sociolinguists have been expanding on this idea, refining the social categories used to divide the population and re-interpreting the role that various facets of identity have on language use. The notions of speech community (e.g., Gumperz 1962), social networks (e.g., Milroy \& Milroy 1985; Eckert 1989) and community of practice (e.g., Lave \& Wenger 1991; Eckert \& McConnellGinet 1992) have been drawn on to better contextualise observed variation, and there has been a more recent drive to use intersectionality (e.g., hooks 1981) as an analytical framework in sociolinguistic research (e.g., Levon 2015).

One of the central tenets of variationist sociolinguistics (e.g., Weinreich, Labov \& Herzog 1968; Tagliamonte 2006) is that language is inherently variable, and this variability is structured in such a way that the constraints underlying this structure can be discovered through multivariate modeling. Such an analysis will uncover not only which factors constrain the observed variation, but how strongly they exert an effect and in which direction (i.e., favouring or disfavouring a particular variant). This multivariate analysis relies on careful coding of the variable tokens, and an exhaustive extraction of all relevant contexts; it is not enough to focus only on the instances when a particular variant occurs, but we must also include all of the instances when the variable could have occurred, but did not. This principle of accountability places some limits on the types of variables that can be studied under a variationist lens, in that it must be possible to fully circumscribe the variable context so that all of the zeroes - all of the places where the variant of interest was not realised - can be counted, and coded.

The variationist field has developed some macro-trends that seem to hold true across speech communities, at least in the Western societies whose data and social structures have informed the development of these generalisations. We will briefly look at Labov's $(1990 ; 2001)$ principles, which have been widely used in variationist sociolinguistics as they make observations on the systematicities of the relationship between language change and gender. We will then consider the generally-poorly defined notion of salience in sociolinguistic research, before finally turning our attention to the 'three waves' of variationist study, characterised by Eckert (2012) as a shift in the 
focus of sociolinguistic research; we will consider how the present study fits into this framework.

\subsubsection{Labov's principles of change}

William Labov's research into sociolinguistic change uncovered some generally robust patterns, which he generalised into principles of language change and gender, and which seem at first glance to create a gender paradox - that is to say, women are sometimes linguistic innovators, and are at other times linguistically conservative. We will explore this apparent contradiction after a quick introduction to Labov's principles.

\subsubsection{Principle I}

The first of these principles looks at stable sociolinguistic variables: variation that exists at the level of the community, but that does not seem to be undergoing any change. A common example is the variable (ing), (e.g., walking vs. walkin'). This variable has at least two surface realisations - the two most common being the velar and apical nasals, although other forms have been attested (e.g., Woods 1999; Fought 2003; Clark 2004). The variable (ing) shows different rates of use across social groups as well as across different speech styles; however, this variability is stable in the community: it has been there for a long time, and the system does not seem to be tipping appreciably in favour of one variant over another.

Principle I: With stable sociolinguistic variables, women use the standard more than men.

With (ing), then, we would predict that women would use the full velar realisation more than men - and this tends to be what has been observed across a number of studies (e.g., Fischer 1958; Woods 1999; Houston 1985).

\subsubsection{Principle Ia}

The second principle also addresses conditions in which women use the more standard variant, although in this case it is when there is a change in progress in the speech community. Importantly, this change must be above the level of awareness: that is to say, members of the speech community are aware of the change, and are able to load social meaning - prestige or stigma, for example - onto the frequent variants.

Principle Ia: With changes in progress that are above the level of awareness, women use the standard more than men.

With variables that fall under the Principle Ia pattern, we find that women use more of the variants that are overtly positively-evaluated in their speech community.

\subsubsection{Principle II}

The third of Labov's principles looks at another situation of ongoing linguistic change in the community, but this time the change is below the level of conscious awareness: when 
speakers are not aware of the different variants in circulation, women tend to be linguistic innovators.

Principle II: With changes in progress that are below the level of awareness, women use the incoming variant more than men.

In this situation, the fact that the variation is below the level of awareness means that there is no overt social meaning associated with the variables.

\subsubsection{Gender paradox?}

The idea of a gender paradox inherent in Labov's principles is sparked by the fact that women are simultaneously linguistically conservative (Principles I, Ia) and linguistically innovative (Principle II). However, there are two perspectives we can take on this apparent paradox that enormously reduce the contradiction.

First, there is the fact that these principles are in a sense complementary: they don't co-occur in the same contexts. The gender effect is overlaid on a map of speaker awareness, which in turn is linked into a network of social meanings. When a variable carries this kind of social meaning, women tend to orient to the higher-prestige variant. That is to say, we could look at the linguistic practice as a reflection of the social context, and note that, for whatever reasons, women seem to be more attuned to the subtle social meanings encoded in speech. We will explore this idea in $\S 1.2 .2$ below.

The second point to consider is that these principles are derived from large-scale studies. While it is possible to generalise that, all things being equal, women tend to act a certain way, the reality is that all things are rarely equal. Which women do we mean when we say women? What effect do other factors such as age, ethnicity, occupation, education, social status, sexuality, etc. have on the homogeneity of the group? Are the women who are conservative with variable $X$ actually the same women who are innovative with Y? Or might they be coming from different subsets of the larger group of people classed as women? Sociolinguists tend to either try to control for potentially confounding factors in their study, or they try to conduct their research in such a way that these confounds are an object of study. That is to say, they will either choose to compare middle-class white women in their twenties with middle-class white men in their twenties (for example), or they will aim to have a diverse enough sample of speakers that the effect of each of these factors can be systematically examined (e.g., participants drawn from middle-class and lower class segments of the population, equally split between men and women, distributed comparably across the age spectrum, and with equal representation of white and Hispanic speakers). The cumulative trajectory of these newer approaches, ones that rely less on aggregating across questionably homogenous groups, is considered in $\S 1.2 .3$ below.

What is interesting, and productive from a language-and-change perspective, is that Labov's principles can be used bi-directionally as an analytical tool. If we know about the speech community, we can infer the status of sociolinguistic variables based on 
the patterns we observe amongst our participants; if a group of young women that we know are linguistic innovators are using a particular variant more than their peers, then we might hypothesise that that variable is below the level of awareness in the community. On the other hand, if we know a lot about the linguistic variable in question, then we can make predictions about which group(s) of speakers are the linguistic innovators, and by extension, about the progress of change. Of course, such strategies rely to a greater or lesser extent on some measurement of awareness, which is in itself a complicated thing to operationalise.

\subsubsection{Salience}

One of the problems with the concept of salience in sociolinguistics is that it rarely given a clear definition. It can be variably used to refer to something linguistic that speakers are consciously aware of (e.g., something they can talk about directly), something linguistic that they are unconsciously aware of (e.g., they are unable to talk about it directly, but it nevertheless correlates strongly with locally-relevant social categories), or indeed something non-linguistic that is foregrounded as relevant in the community (e.g., ethnicity may be immediately relevant in a given community, but people may not have made explicit links between ethnicity and particular linguistic features) or in the interaction (e.g., growing up in different neighbourhoods of the same city may become relevant when particular topics are under discussion, but may be nonrelevant at other times). These differences in definition partly reflect differences in research interests: if you are investigating the interactional emergence of shared identity, for example, then you will draw on a different notion of salience than if you are investigating the diffusion of a phonological change across a speech community.

However the term salient is defined, it is clear that different linguistic variables will behave differently depending on the degree to which they carry social meaning in the speech community (e.g., Trudgill 1986; Kerswill \& Williams 2002). The domain of the social meaning (e.g., intra-speaker, inter-speaker, between speech communities, etc.), and how that social meaning is manifested (e.g., style shifting, accommodation, divergence, etc.) will depend on the particulars of the research topic, the speech community under investigation, and the researcher doing the analysis. In variationist sociolinguistics, salience is often operationalised in terms of how speakers use particular variants across different speech styles.

\subsubsection{Indicators, markers, stereotypes}

Labov (1972) makes a useful distinction between indicators, markers, and stereotypes. Indicators are variables that index broad demographic categories such as age and "social distance from the originating group" (1972: 320), but are under the radar - that is to say, people are not aware of variability, and they do not seem to be loaded with social meaning. Markers show an effect of style shifting, suggesting that they have "more 
systemic social value" (1972: 320) and that people are aware that there are more and less prestigious variants. Stereotypes are linguistic variables that are fully accessible to the speech community, are "discussed and remarked by everyone" (1972: 320) and are overtly associated with particular groups of speakers.

Using this typology of linguistic variables, then, we can draw parallels between Principle II and indicators (i.e., variation that speakers are unaware of) and between Principle Ia and markers (i.e., variation that speakers are aware of). Stereotypes must be a special case of the Principle Ia variables, in that speakers are aware of them to the extent that they can explicitly comment on them.

\subsubsection{Salience as gradient}

It follows, from our previous observation that stereotypes are more accessible than markers, that salience must be gradient: some things are more salient - however we are defining salience - than others. Analytically, we might prefer salience to be categorical and binary, because identifying variables as salient/not salient makes for a more straightforward quantitative analysis. But to try to force this either/or structure onto the complicated concept of salience seems to be doing a disservice to what salience actually gives us, which is a sense of people's level of awareness of something. At the very least, our level of awareness of the world around us is tri-partite: things we are completely unaware of, things we are dimly aware of, and things we are acutely aware of.

Of course, this is still a categorical distinction, but at least it is not a binary one: it admits an intermediate state, and provides a better mapping onto Labov's indicators, markers, and stereotypes. However, the question then becomes: where are the boundaries between these three states? If I am outside on a summer evening, at what point do I go from having a background awareness that there are mosquitoes in my back yard, to having a very focused awareness that I need to go inside? Is the difference between the two states purely quantitative? Is there a threshold of mosquito density below which they are an annoyance, and above which they are intolerable? Or is the difference more qualitative: when they are buzzing they are an annoyance, but once they start biting they become a problem?

To bring this back to the question of linguistic salience: is the difference between markers and stereotypes a quantitative one - once we hear variant $\mathrm{X}$ so many times from a particular group of people, do we start to make a social association? Or is it more qualitative - are some linguistic features, or some domains of linguistic variation, inherently more accessible to speakers than others? Certainly we notice some linguistic differences more than others - different accents, or different words - but it's not clear whether these are linguistically or socially more noticeable. Accent differences are largely phonetic (segmental and/or prosodic), but they are also composite: which of the many phonetic differences are we actually registering when we decide that someone has a different accent to our own? Lexical differences can lead to misunderstandings that are 
entertaining (thong refers to very different items of apparel in Canada and New Zealand, for example), so again it's not clear whether they are so noticeable because the differences are lexical (linguistic) or because they're funny (social).

It is also important to note that Labov's use of the term stereotype is a linguistic one (those people say X) rather than a social one (those people are Y). Although the two concepts are doubtlessly closely linked (those people are Y because they say X, or they say $\mathrm{X}$ because they are Y), it is important not to conflate them. We will look briefly at social stereotypes in section $§ 1.3 .2$, below.

\subsubsection{Salience in this project}

Throughout the various discussions that follow, we will adopt a very light definition of 'salience', and use it as a shorthand for the degree to which a linguistic form carries interpretable social meaning at the level of the community - that is to say, the extent to which a linguistic form is likely to encode social meaning (in particular, gender) in a way that is accessible to members of the speech community, whether consciously or unconsciously. This definition is deliberately vague with respect to how salience happens on the perceptual side of an interaction, as this question is better explored through psycholinguistics than through variationist sociolinguistics. Nevertheless, we need some term that we can use to talk about social meaning from the production side of things, and salience, in its broadest sense, seems like a good candidate.

When we use salience in this thesis, then, we are drawing on Labov's typological classification of variables, but we are also developing an analytical approach to assessing this interpretable social meaning through an examination of the linguistic choices made by people who have crossed the social category boundary between masculinity and femininity. These liminal people (see $\$ 1.4 .4$, below) can provide valuable insight into the social interpretability of distributional gender differences in the variable analyses that comprise chapters four, five, and six; this perspective in turn allows us to better understand the extent to which those variables may (or may not) be useful resources in presenting a particular gendered identity in Auckland.

\subsubsection{Eckert's three waves of variation studies}

Eckert's (2012) classification of the trajectory of variationist research into three distinct waves, each with its own focus, has had a profound effect on how we think about the approaches and objects of study in variationist sociolinguistics. Studies characterised

by Eckert as first wave tend to explore broad correlations between linguistic variables and macrosocial categories such as class, gender, ethnicity, etc. Quite often the object of study is the language system itself (particularly looking at phonological and morphosyntactic variation), and the social contexts are presented as explanatory factors in the spread and uptake of structural change. Critical responses to the social generalisations drawn from these first wave studies gave rise to Eckert's second wave of 
variationist research, where the focus has shifted more to locally-constituted social meaning. These second-wave studies are heavily contextualised through careful ethnographic observations, and the notion of identity as a driver of linguistic variation has been foregrounded. Both the first and second waves share the ideological perspective that linguistic variation marks social categories in the speech community; in contrast, the perspective characteristic of Eckert's third wave of variationist sociolinguistics is that this same variation constructs social meaning, rather than simply reflecting it. In the thirdwave tradition, the focus of study is style: how individuals make strategic use of linguistic resources to dynamically present their identities across a shifting interactional landscape.

As the taxonomy suggests, the three waves can be thought of as having arisen more or less in sequence ${ }^{1}$, with critiques of one approach driving the development of the next. This progression makes sense if we consider all three waves as happening in the same speech community: each wave builds on the findings of the previous, working towards a more complete understanding of the linguistic practices of the people comprising the speech community. But when the third-wave approach is adopted without a coherent understanding of the community norms in which stylistic practice is taking place, then the (social) interpretation of the observed variation runs the risk of being too speculative, of relying solely on the researcher's intuitions about the associations that exist at the level of the community.

\subsubsection{Contextualising the third wave}

Social interpretation of linguistic behaviour doesn't happen in a vacuum: it happens in the context of what people have experienced, and the associations they make between types of people and particular practices. Labov (2001) argues that sociolinguists need to understand variation at the level of the group before trying to explain it at the level of the individual. Humans are good at recognising patterns, and ascribing meaning to them our capacity for pattern-recognition is thought to have given us an evolutionary advantage (e.g., Jordan 2013) as well an unfortunate tendency towards magical thinking and conspiracy theories (e.g., Mattson 2014; Shermer 2008). If you meet one red-headed person who sneezes a lot, you might not read much into it beyond the idiosyncratic: she has red hair and she sneezes. But if you meet ten red-headed people and they all sneeze a lot, you might begin to correlate red-headedness and sneezing. We extrapolate over probabilities and trends to make meaning at the level of the group, which then allows us to interpret individual behaviour.

Without a clear understanding of what happens at the level of the speech

\footnotetext{
${ }^{1}$ Although Eckert (2012) notes that Labov's study of (ay) and (aw) in Martha's Vineyard examined variation in an ethnographically-embedded context, she notes that subsequent research "moved quickly away from social meaning to focus on macrosociological categories as they reveal (and presumably structure) the spread of linguistic change through time and space" (p.88).
} 
community, then - the level at which we make meaningful associations - interpreting linguistic style as an index of social associations presents a relatively weak argument. If the variable in question is a stereotype, or has been previously documented and explored with respect to social indexicalities, this link is fairly unproblematic. Podesva's (2007; 2008 ; 2011) work on the social performances of identity of gay male professionals in the United States is a prime example. There is (presumably) enough general awareness of what a professional identity or a diva identity entails in the US - and how various linguistic resources can be drawn on to perform these identities - that the links do not need to be made explicit. Podesva is thus able to claim that a participant's use of "phonetically strong stop releases signal prissiness" (2008: 137), and that this prissiness is further associated with a gay diva identity, without having to provide evidence that people in the speech community actually interpret strong stop releases as 'prissy', or that they bundle 'prissy' in with a gay diva persona. For anyone even passingly familiar with US pop culture, for example, these associations are intuitively obvious.

Similar links are made by Besnier (e.g., 2003; 2004) in his linguistic and anthropological work on the use of English in Tonga, where brief, isolated pieces of data are put forward as representative of larger-scale linguistic constructions of social structures. This is on some level unavoidable - to fully contextualise each item would be an exhausting and never-ending exercise - but the fact that the average person is likely to be less familiar with the nuances of Tongan identities than they are of those of American professional men means that we have to take to it on trust that Besnier's interpretation is an accurate reflection of how these items would be interpreted in the Tongan context. Where Podesva is working with a widely-accessible set of identities, Besnier is focusing on a less globally-broadcast cultural context, but the structural assumptions of their arguments is the same: $\mathrm{X}$ means $\mathrm{Y}$ because it happens in context $\mathrm{Z}$, but the reliability of the indexical networks associated with $\mathrm{X}$ and $\mathrm{Y}$ in context $\mathrm{Z}$ is, to a certain extent, taken for granted.

This is not intended as a criticism of either Besnier's or Podesva's research: they are both pursuing research very much in line with the third-wave orientation to style as the micro-scale locus of identity performance, and they are both extremely adept and well-respected researchers in this area. But if the structure of the argument is problematic for Besnier, then it must also be problematic for Podesva, because neither presents a coherent argument that the interpretation that they are putting on the data is the same one that the speech community does. As a quantitative researcher, I find this inherently unsatisfying; I would like evidence that their interpretation is sound and valid. I am not claiming that either of them is wrong - certainly Besnier, coming from a more anthropological perspective, has a good depth to his cultural understanding of Tonga but rather that their arguments omit much that is not accessible to someone unfamiliar with the cultural context, and they therefore seem a bit weak.

With this in mind, then, this project is designed more in line with Eckert's 
second wave. It builds on the previous research conducted on variation in New Zealand English (much of which has been driven by an interest in the sound system itself) and explores the local construction of gender in a particular speech community. In mapping this local terrain, we will be laying the groundwork for future third-wave studies of gendered identity in Auckland, which will rely on some kind of baseline as a reference point (c.f., Eckert 2000; Meyerhoff \& Stanford 2015).

So far, we have looked briefly at some of the major findings from variationist sociolinguistics that will inform the analyses that follow, and we have proposed an approach to handle the hard-to-pin-down concept of linguistic salience. Linguistically, at least, we have a sense of the traditions that this project will build on; we will now consider the social concepts that underpin our approach.

\subsection{Making meaning}

The previous section laid out some of the big-picture linguistic reasoning that has informed this project. In this section, we take a brief look at the corresponding social reasoning that has also informed the development of this project. This addresses not only the specific question of how we make meaning out of the linguistic input that surrounds us, but also how we make meaning of the world more generally, and of gender in particular.

\subsubsection{Social constructionism and critical realism}

An in-depth analysis of the social constructionist and critical realist perspectives on meaning-making is beyond the scope of this project, but it worth introducing them briefly, as they directly interrogate the assumptions that we may have about the world that we live in.

Social constructionism can be characterised as a philosophical position that questions the naturalness of the categories we use to understand the world around us (Burr 2015: 2-5); rather than taking these categories (e.g., man and woman) as preexisting in the world, social constructionism argues that these are socially constructed artifacts, and as such are historically and socially specific. What it means to be a man in 2017 in Auckland is different than what it meant in Auckland in 1917, or indeed in 2017 in Port Moresby, Papua New Guinea. Or, in fact, what it means in the ethnically and socioeconomically different regions of Auckland, such as Devonport (predominantly of European descent) and South Auckland (largely Pasifika and Māori).

This is not to say that there are no commonalities between these various definitions: western schools of thought tend to default to biological categories as a basis for determining gender, and although some scholars argue that biological sex is also a matter of social construction (see e.g., Butler (1990) from a queer and feminist 
perspective, and Fausto-Sterling $(1993,2000)$ from a biological and gender-studies perspective), for many people male and female are (a) exhaustive, and (b) pre-existing 'natural kinds'. Certainly there are strong correlations between biology and the types of people who identify as - or perhaps are identified as - males and females, but this does not mean that anatomy and genetics themselves are wholly deterministic. Burr (2015) points out that things thought of as natural kinds are often packaged as being "unchangeable and unchallengeable" (pp.111-112), and therefore attempts to change or challenge them are met with hostility. Consider the anger currently being stirred up in the USA around trans people's use of public washrooms, for example: people appeal to the 'unnaturalness' of 'men in dresses' using the same toilet as young women. Their unwillingness to acknowledge the identity of trans women as women speaks to their rigid adherence the natural classes of men and women.

Language is central to the social constructionist position, as it is through interaction that these social artifacts are co-constructed and reified. Critical discourse analysis (e.g., Wetherell 1998; Fairclough 2015) draws heavily on social constructionism to interpret language exchanges, not only with regard to what is said, but also to how and when it is said. Gender is not simply a matter of biology, but also of how we talk about and socially co-construct - what it means to have a 'male' or 'female' body, in this time and in this place.

Critical realism could be said to offer a compromise with respect to the extreme interpretation of the social constructionist position that nothing exists independent of our descriptions of it. Burr (2015) notes that "Critical realists believe that although our perceptions and sensations do not mirror reality [...] they are not independent of it" (p.108). That is to say, things $d o$ exist in the real world, but our understanding of them is mediated by our cultural experiences and expectations. The social construct of gender can exist at the level of the community, even though we experience it as individuals.

This is the position that we will adopt for this study. As I see it, masculinity and femininity exist within the culture of Auckland in 2017: although the particular characteristics of men and women are negotiable (and are arguably undergoing some kind of structural reanalysis within the community, in the sense that women have more freedom with respect to the course of their lives than they would have had thirty years ago), people generally have a stable enough sense of masculinity and femininity that they are able to identify some things as masculine and others as feminine. Although at a deep level gender is not a natural kind - existing in the real world independent of our constructions of it - at a superficial level, people treat it as though it is.

These stable points of gendered consensus are not necessarily consistent across the community - indeed, we would expect there to be differences between how men and women see masculinity and femininity, for example, or differences correlating with age, or between ethnic and cultural groups - but there is some broad agreement on the types of human beings that are classed as men, and those that are classed as women. Queer and 
trans people may challenge these categorisations, and may be hard to classify in the either/or system that individual people use to divide up the world, but they do not in and of themselves destabilise the gendered order to the extent that the social categories of men and women are no longer meaningful for the majority of the population.

\subsubsection{Prototypes and stereotypes}

If we acknowledge that there are differences between individuals as to whether or not a particular behaviour is clearly recognisable as masculine or feminine, but that there are nevertheless broad patterns of masculinity and femininity that are accessible to the population at large, then we are in a sense arguing for the existence of some kind of gendered prototypes: clusters of attributes (e.g., Rosch 1973; 1978) that in some way encompass the essence of masculinity and femininity, and that we use as a model for comparison in assessing any individual's gendered presentation.

While we may be hard-pressed to characterize prototypical masculinity and femininity (see Visser 1996, 2002 for one approach), the existence of such prototypes is hard to deny. If queer speakers are creating gendered identities in opposition to stereotyped expectations - either subverting those expectations for their own purposes, or ignoring them completely - then there must be coherent and broadly recognized standards of masculinity and femininity: in order for something to be transgressive, there must be something that is being transgressed. At least at the level of the community, masculinity and femininity have some agreed-upon meaning, with different expressions of masculinity and femininity rated as more or less prototypical.

In fact, we can extend the notion of prototypicality away from the mainstream, and propose homonormative as well as heteronormative gender prototypes.

Heteronormative masculinity and femininity need no particular justification: they are the default models for doing gender in western societies. The existence of corresponding homonormative prototypes, on the other hand, may be less obvious. But consider the widespread stereotypes of gay men and lesbians, respectively. The idealized gay man may be an effeminate, limp-wristed gym bunny who dresses well, watches his weight, and talks with a lisp. The idealized lesbian might be a butch woman with short hair and asymmetric piercings, who dresses like a hipster boy, and is more concerned with her health than with her dress size. Of course, neither of these constructions is real - no gay man or lesbian is expected to embody these stereotypes in their entirety - but these are the norms against which one's queerness is assessed. This exactly parallels heteronormative prototype assessments: the farther you stray from your gender prototype, the more marked your identity is. Within a heteronormative framework, effeminacy would be seen as unfelicitous in men; within a homonormative framework, it would be unfelicitous in women.

Of course, the queer prototypes proposed above smack of stereotypes: haven't I simply listed a collection of stereotypical associates about queer men and women, and 
called it a prototype? Geeraerts (2008) proposes, in fact, that stereotypes and prototypes are necessarily closely linked, claiming that "stereotypes are prototypes from a social angle" (p.27). Thus the prototype is the mental ideal that we have as individuals, and the stereotype is the collective averaging-out of these prototypes across the population - a "link between the psychological and the social organization of semantic knowledge" (Geeraerts 2008: 27). Prototypes can vary widely between individuals, depending on their direct experience with the people who constitute the category in question, without it necessarily changing the social stereotype. So people who spend a lot of time with queer men, for example, may have a different prototype of queer masculinity than people who don't, because of the types of queer men they actually interact with, but if the majority of the population has limited contact with queer men, then theirs are the prototypes that dominate the social stereotype. Stereotypes can change, however, as prototypes shift over time; the social stereotypes of women and men, for example, may be different between generations, as younger Aucklanders have had a collectively different set of individual experiences of masculinities and femininities - and of queer and straight people - than previous generations.

\subsubsection{Strategic essentialism}

It is almost inevitable that a study such as this one will be criticised for the categorical way that it treats people's identities. After all, I am in a sense presupposing categories of analysis, and binning my participants in a way that is analytically convenient, at the risk of ignoring all of the individual variation within and between speakers.

In one sense, yes, this is exactly what I am doing: the focus of this study is on the linguistic presentation of gender at the level of the community, and the ways that social meaning accretes through the cumulative linguistic practices of the speech community as whole. This kind of question necessitates an abstraction away from the level of the individual, and towards socially recognisable groups, which is the approach taken in this project. This is not to say that any group of queer women, for example, is in some way inherently homogeneous; we would expect to find the same range of individual variation here as anywhere else. However, it is important to stress that this categorisation of participants as queer or straight is not a product of my analytical interests: it is driven by their own identities, and their own social practices. The queeridentified participants, for example, were not classed as queer because of their sexuality per se, but rather because of their engagement with queer communities, and their own inclusion of their sexuality as part of their self-defined identities. This is discussed in greater detail in $\$ 2.3$, where the particulars of participant recruitment and categorisation are explained in more depth.

In a sense, I am engaging in strategic essentialism (e.g., Spivak 1984-85; 1990: 109) albeit in a way that Spivak herself would perhaps be unhappy with. Strategic 
essentialism recognises the many faults underlying essentialism, but acknowledges that at times it is a useful strategy for minorities and oppressed groups to unite behind a common cause. The organising behind the 1980s Homosexual Law Reform in New Zealand (see §2.2.3), for example, drew disparate groups of lesbian, gay, and bisexual (LGB) people together, in spite of their many differences, to focus their collective energy on a particular fight. Spivak's objection to the extension of strategic essentialism to include out-group essentialism (i.e., someone from outside the group deciding that part(s) of the population can be treated as homogenous) is essentially that it legitimises essentialism as a whole, and can therefore be used as a tool of oppression rather than a defence against it. In that sense, Spivak would not approve of my use of the term strategic essentialism, as the queer participants in this project have not explicitly identified themselves as a cohesive group.

Nevertheless, to conduct the research that I am interested in - an investigation of the sociolinguistic presentation of gender within a speech community, conducted in a second-wave framework (see $§ 1.2 .3$ above) - certain generalisations need to be made. In making these generalisation, I am not claiming that all queer women or all queer men are the same, or indeed claiming that they would classify themselves as a unified group under most circumstances. New Zealand in the 1980s did see a coming-together of gay men and (some) lesbians to present a united front in the face of social opposition to the decriminalisation of homosexuality, in spite of considerable inter-group differences and tensions. This is the kind of context in which Spivak might endorse the use of strategic essentialism, in that the individuals being essentialised have grouped themselves together for their own purposes. In the context of this study, although the individuals in question self-identify as lesbian, gay, or queer (i.e., their non-heterosexuality is an important part of their identity), they do not self-identify as a group.

However, inasmuch as there exist socially-recognisable categories of queer women and queer men, then the prototypes and stereotypes of these categories in the wider community are likely to have been drawn from generalisations that parallel those that I am making here. In that sense, my queer participants are jointly engaged in coconstructing those categories, regardless of whether or not they are doing it cooperatively. As we will see in $§ 7.6$ as well as in the individual analyses throughout this thesis, this approach is not unwarranted: gendered identity as operationalised in this project turns out to be a significant predictor of the observed variation, under multivariate analysis.

\subsection{Bridging the linguistic and the social}

So how then do we connect the social identities and gendered categories with the linguistic practices that create and constrain them? There are two related processes that 
will prove particularly useful for this endeavour: enregisterment, the mapping of social meaning onto linguistic form; and indexicality, the interpretation of social meanings from linguistic form. These can be combined as a linguistic performance of gender in Butler's (1988; 1990) sense of 'performance', and the way that the Auckland Trans Corpus of sociolinguistic interviews has been constructed (see $\$ 2.3$ for more detail) gives us the opportunity to carefully explore the links between language variation and social identity, with a particular focus on what I will call liminal identities.

\subsubsection{Enregisterment}

Broadly speaking, enregisterment is the "process through which a linguistic repertoire becomes differentiable within a language as a socially recognized register of forms" (Agha 2003: 231). Agha develops a model of enregisterment through a careful study of the social valuation of Received Pronunciation (RP) in Britain, and points out that the processes of "valorization and circulation" (2003: 232) that he describes are generalisable to other linguistic phenomena in other social contexts. We will briefly look at these processes that Agha highlights, combining some in ways that make them more applicable to the question of gender rather than dialectal differences, and discuss how gendered linguistic practice may have become enregistered over time in New Zealand English.

\subsubsection{Asymmetries of competence and register complexity}

In discussing RP, Agha points out that everyone in Britain knows what RP sounds like, but a very small portion - perhaps $3 \%$ of the population - actually has a full command of the variety (2003: 234). This is partly because what Agha calls its phonolexical register, by which he means that the characteristics of the variety, i.e., "knowing the precise lexical boundaries within which particular phonological rules apply" (p.235), is hard to emulate consistently for non-native speakers.

This idea is extendable to the question of gender and linguistic performance as well: we are all certainly aware of differences in how women and men talk - or indeed how gay men and straight men talk, and to a lesser extent we can identify some lesbians as sounding lesbian - although this does not mean that we can confidently explain what those differences are. Linguistic stereotypes are fairly accessible, but limited, and often not all that accurate; they are based on a handful of particularly salient features, and overextrapolated to more general cases. This is similar to the native speaker of RP who knows that glass and grass share the same vowel, while crass and gas have another, but someone naively putting on an RP accent may reasonably (and incorrectly) assume that the four words are in the same lexical set. To an outsider, the mistake may pass unnoticed, but not to the insider.

In a sense, this asymmetry of competence and a register's complexity of 
realisation compound to produce an evaluative authenticity: that is to say, native users of a register who hear that register being used can generally tell whether it is an act that someone is putting on, or if it is a genuine performance of authentic identity. This is true at least for the more marked gendered varieties, such as a camp male persona, or a young urban-oriented woman. Camp men can evaluate the authenticity of someone who is 'doing being' camp (and so exert a kind of social pressure to conform on those who are getting it 'wrong'), and young women can assess the authenticities of various styles of young womanhood, in ways that outsiders can not.

\subsubsection{Awareness and evaluation of difference}

It is not enough that differences are noticed, but they must also be differently evaluated. This evaluation is not restricted to the linguistic differences, but should also extend to include characteristics of the people who use a particular variety. This is not a new concept in sociolinguistics: dialectologists and sociolinguists concerned with language attitudes have long made use of these kinds of associations in the minds of members of speech communities (e.g., Rickford 1987). In the case of RP, it is the language of the elite, and so it carries all of the emotive and sociocultural baggage that goes with this kind of elitism: respectability, privilege, and money on the part of the elites, but also a degree of being useless, clueless, and out-of-touch.

As we will see elsewhere in this thesis (see $§ 7.4 .1$ ), the linguistic habits of younger women are often criticised, and by extension, so are the young women themselves. Traditional images of womanhood (for example, being nurturing and caring, showing compassion and empathy, being generally submissive and deferential) are often eschewed by younger women in favour of a more fully autonomous self, which can include being assertive, having fun at parties, and exercising their own sexual agency. These new ways of being a young woman tend to get reinscribed by hegemonic observers as evidence of moral failings: they are bossy/bitchy, irresponsible, and being shameless sluts. It should be noted that these external interpretations are inherently gendered, because these same qualities or behaviours on the part of (straight) men are not generally cast in this kind of negative frame. Queer men can also be painted with this same brush, and often with the same paternalistic overtones of control: these are bad and dangerous things for you to do, so you should stop doing them for your own good.

It is interesting to note again that there is an insider/outsider dichotomy observed here, that parallels what we saw with the question of authenticity above, although its polarity (as it were) is flipped. Young women who are assertive party animals, for example, do not share this negative view of their lifestyle, and these same qualities can be seen as positive attributes in some male subgroups in the queer community; it is outsiders who pass judgment. With the question of authenticity, it is the insiders whose judgment matters; with the question of respectability, outsiders preside. These groupings, and the evaluations that surround them, are policed from both sides. 


\subsubsection{Evaluations are disseminated and embraced}

Finally, the awareness of difference and the web of social evaluations that surround these differences have to move through the community. Agha (2005: 249-260) shows how increasingly accessible accounts of RP (starting with small-circulation prescriptivist works restricted to the aristocracy and intelligentsia, and culminating in mass-produced and -consumed 'penny weeklies' accessible to everyone) contributed to the spread of RP as the de facto variety of the elite. Certainly, it is uncontroversial to say that the speech of women, and the social role of women more generally, has a long history of being criticised by those in power. So although the specific targets may have shifted over time (for example, it is no longer scandalous that a woman should appear in public wearing trousers, or work outside of the home, although these examples are inextricably bound up in issues of class as well as history), the fact of women bearing the brunt of a critical male gaze is hardly a new one. It is also widespread and deeply ingrained: even very young children can be observed reverting to stereotypes and traditional social configurations.

Because this critique of femininity is so long-standing, it is difficult to trace anything like a chain of events; this is less so for the social valuations of queer people, who have only relatively recently become a visible part of public society. The fact of men having sex with other men, and women with other women, is attested throughout history, and evaluated differently in different societies. Morality, religion, and politics in the English-speaking world effectively drove the behaviour underground, where it existed well below the radar - and was therefore not subject to public discourse - until, arguably, the trial of Oscar Wilde in 1895, which dragged an entire subculture into the public eye. It was assessed and deemed abhorrent, not to mention criminally immoral, and the association between homosexuality (a relatively new term at the time) and effeminacy, theatre and the arts was established (O'Connor 1998: 108). Homosexual men were deviants, but they were identifiable through their hobbies, habits, and friendships; a new reason for, and means of, policing masculinity was born.

The social context of male homosexuality in the English-speaking world has changed dramatically over the course of the last century, and is well-documented elsewhere (e.g., Duberman, Vicinus \& Chauncey 1989). For our purposes, it is enough to note that, in New Zealand, it has been denied, detested, tolerated, accepted, and finally mainstreamed. At each of these stages, attitudes towards queer men (and women, although in many ways female homosexuality is often less marked and explicitly condemned than its male counterpart) have changed in tandem with the political and social stance, to the point that the young people in the ATC find queer identities neither exceptional nor strange.

Under Agha's model of enregisterment, the medium of dissemination of this bundle of attitudes and evaluations can include mass media, meaning that attitudes can be 
influenced through widely available popular culture outlets such as books, magazines, film, and television. Public representations of queer men and women have changed over the past few decades, becoming less marginalising and arguably more accepting (or at least more notionally tolerant) of sexual diversity. There has also been a dramatic change recently in the media representation of trans people, and although the conversation is often couched in socially conservative terms (e.g., moral panic about gendered public spaces, such as toilets and changing rooms), the public nature of these discussions at least acknowledges trans identities, and the fact that trans people are already part of the larger community. It is too soon to make any confident predictions about how accepted trans people will be in society generally - particularly those who identify as something other than recognisable instantiations of women or men - but the early signs seem promising in New Zealand. So while the public perceptions of queer and trans identities are not identical, attitudes towards both are being influenced at least in part through media representations.

Agha's model for enregisterment, for relating social meaning to people's linguistic practice, is fairly straightforward, and can be applied unproblematically to the relationship between gendered identity and language in New Zealand. But this is only half of the story: we have not yet addressed the question of how we interpret social meaning from the language around us.

\subsubsection{Indexicality}

The notion of indexicality is not a new one in linguistics, but it has been adapted for use in sociolinguistics and linguistic anthropology, particularly by Ochs $(1990 ; 1992)$, Silverstein $(1985 ; 2003)$ and Eckert (2008). In the sociolinguistic context, indexicality refers to "a property of speech through which cultural contexts such as social identities (e.g. gender) and social activities (e.g. a gossip session) are constituted by particular stances and acts" (Ochs 1992: 335). There is a distinction made between direct indexicality - what a linguistic item literally refers to - and indirect indexicality - the web of associations that we have with that linguistic item. This web of associations is dynamic and non-linear, with meanings that can shift over time, and results in a complex indexical field or "constellation of ideologically related meanings, any one of which can be activated in the situated use" of a linguistic form (Eckert 2008: 454).

This is a departure from the more traditional view that equivalent forms are simply different ways of saying the same thing; through the different indexicalities of these competing forms, each one invokes different attitudes, stances, and ideologies. For example, the sentences in (1) are, in one sense, equivalent: they are requests - couched in different terms - for some fresh air.

(1) (a) Open the window.

(b) Could you open the window, please?

But in a more meaningful sense, they are doing very different things sociolinguistically. 
The brusque tone of (a) indexes a power dynamic in which the speaker is licensed to give explicit instructions. The more polite form in (b) acknowledges the imposition of making a request, and tries to mitigate it through the use of the modal could, the word please, and phrasing it as a question. The relationship between the speaker and the addressee is different in the two examples, and our previous experience with each of these forms predisposes us to imagine a particular type of person in each case. That is to say, each request-making strategy has its own indexical field in our minds, linking the linguistic form to the associations that we have with it, and triggering certain expectations. What those expectations will be depends not only on our idiosyncratic experiences, but also on other pragmatic and contextual factors. The form used in (a) for example could be indexing authority or power, or could alternatively be indexing an intimacy that has been stripped of its need for indirectness or politeness. Which of these associations will win out is contingent on the type of relationship that we perceive between the two people who are interacting. Both (a) and (b) are part of the same indexical field, but they have different meanings and are interpreted in different ways.

The model of enregisterment (see §1.4.1) comments generally on how meanings can become associated with linguistic forms, but ordered indexicality (Silverstein 2003) gives a more precise explanation for the shifting nature of these meanings, by way of reinterpretation and reinscription. To continue with the example (a) above, we may consider our two associations - power and intimacy - to be distinct, but they are related by a third association: sincerity. If the relationship between us is hierarchical or antagonistic, I may not feel compelled to put any effort into being polite with regards to the window situation, as that politeness would clearly be insincere and pandering on my part. This principle (politeness=insincerity) could then be extended to the more intimate context, where (for other reasons) I do not wish to come across as insincere. In Silverstein's model, the first instance - where I can't be bothered being polite - is the (n)th order of indexicality: a well-established association of form and meaning. The second instance - where I have extended the association to encompass a new and substantively different context - is the $(n+1)$ th order of indexicality. That is to say, I have taken an existing index - the (n)th - and taken it one step further - the (n+1)th.

What is important here is that this extension - the move from (n) to $(n+1)$ - is not only possible, it is in fact inevitable (Silverstein 2003: 212): people will always reinscribe and reconfigure existing resources for new uses, whatever those resources are. Eckert (2008) presents an interesting comparison between linguistic repurposing and other types of reinscription, and finds that people - particularly young people - are highly adept at consciously deconstructing composites such as fashion, choosing one or two elements of the composite, and using them in new ways, which reinscribes meaning onto those parts.

Linguistic forms may be harder to decompose as explicitly as fashion, and indeed the process may be much more unconscious with language than with other 
stylistic options, but there is evidence that speakers nevertheless retool linguistic resources to meet their communicative and social needs. Podesva (e.g., 2007; 2008; 2011) explores the indexicalities of several variables in the construction of gay male professional identities in California, and shows that the same linguistic resources - for example, $/ \mathrm{t} /$ release or the use of falsetto - can be deployed by the same speakers in different ways and different combinations, across different contexts, to construct different composite identities, such as professional or diva. His participants are drawing on the different sets of associations for each variable - the various elements of their indexical fields - to construct, moment-to-moment, an appropriate persona. As noted above (see $\S 1.2 .3 .1)$, however, there is a risk of circular reasoning in this approach unless the interpretation is grounded in evidence of how the speech community interprets these cues.

It is perhaps worth mentioning at this point that this discussion of indexicality and indeed, the thrust of the literature on sociolinguistic indexicalities - has focused on the agentive (re)appropriation and (re)inscription of meaning of various linguistic forms: in essence, it is presented as a trajectory of adoption. If I notice a member of group $\mathrm{X}$ using linguistic form $\mathrm{Y}$, and $\mathrm{I}$ have an association between group $\mathrm{X}$ and some positivelyevaluated quality A, then I may adopt and adapt $\mathrm{Y}$ (call it $\mathrm{Y}^{\prime}$ ) as a way of appropriating some of the A-ness that I admire so much. And once I have done this, then it becomes possible for someone to look at my doing $\mathrm{Y}^{\prime}$, think that I personify the much-admired trait B, and make Y' their own, and so on. However, this does not mean that indexicality is unavailable for avoidance work. If my impression of group $\mathrm{X}$ is that they are horribly C-like, then I may choose to adopt some form of anti-Y $(\neg \mathrm{Y})$ as a distancing strategy: I'm so non-C-like that I use $\neg$ Y. From there, $\neg \mathrm{Y}$ becomes an available $(\mathrm{n}+1)$ th order indexicality for further re-inscription, either from someone who admires my $\mathrm{C}$ qualities and develops $\neg Y^{\prime}$ as a measure of adoption, or who so despises those same $\mathrm{C}$ qualities that they respond with $a \neg(\neg \mathrm{Y})$, and arrive indirectly at a re-inscribed $\mathrm{Y}^{\prime}$. At each point in the re-inscription of meaning for a linguistic form, then, speakers are able to orient to either a positive (adoption) or negative (avoidance) strategy (cf. Bucholtz 1999; Meyerhoff 2001) within the framework of the indexical field. We can think of these as instances of indexical convergence and divergence.

For the most part, this thesis is not directly concerned with the particular indexical chains that constitute the meaning-making enterprise; we are more interested in the endpoints of those chains - the correlation that group $\mathrm{X}$ tends to do thing $\mathrm{Y}$, for example - and in assessing through liminality whether this correlation is likely to carry social meaning. A thorough investigation of these chains of indexical relationships is better-suited to a third-wave analysis, which in turn relies on the endpoints of these chains of meaning having been identified and established. The mapping of these endpoints in this project, and the process of determining which of them are likely to be doing something socially interpretable, will enable future third-wave studies of style as a 
linguistic repertoire in this speech community.

\subsubsection{Gender as performance}

With linguistic forms inscribable with social meaning, and with social meaning interpretable on linguistic forms, we arrive at a sense of linguistic practice being a kind of performance: we speak in a certain style so that our social identities will be understood by our interlocutors in a particular way. Whether or not this performance is successful that is, whether or not people interpret our intentions as we would like them to - is an open question, but does not detract from the actual performative nature of presenting an identity through language.

Butler (1988: 519) has characterised gender as a performance in the sense that it is "an identity tenuously constituted in time [...] instituted through a stylized repetition of acts". That is to say, gender is something that we do, rather than a static property that exists independent of our actions. Of course, we need some degree of shared framework for interpreting such a performance, and this framework takes the form of stereotypes (at the social, community level) and prototypes (at the individual, psychological level) that we can use to evaluate the gendered performances around us. Our linguistic practice can align with these stereotypes, and act to reify the status quo, or our linguistic practice can distance us from them, and work to create alternative social categories.

The gendered landscape in which this project is situated allows for more than one monolithic masculinity and one monolithic femininity. We are categorising men and women according to their investment in heteronormative or homonormative (i.e., queer) gender orders. We are also allowing for the possibility that the organisation of these gender orders may have changed or realigned during the socially turbulent 1980s in New Zealand, by including a cohort of speakers who came of age - and developed much of their gendered social identities - before these sweeping social changes, and a second cohort of speakers who came of age in the aftermath. We do not know at the outset what effect these social changes will have on the linguistic system of Auckland English, if indeed it has any, but by including two age groups that straddle this time period, we are able to ask the question in an answerable way.

\subsubsection{Trans identities as liminal}

As well as including queer and straight speakers, the ATC includes a group of participants for whom the performative nature of gender is potentially made more explicit. Several trans men (female-to-male transsexuals, i.e. people born and socialised as women, but who identify and live as men) and trans women (male-to-female transsexuals) are included in the ATC, which allows us to investigate the degree to which the gendered nature of language is malleable in adults.

There are a range of identities that tend to get swept up under the term 'trans', which can range from part-time transvestitism (cross-dressing) through permanent gender 
reassignment (transsexuality) and into non-binary identities that eschew the notions of binary gender, or indeed fixed gender identities (genderqueer, genderfluid, genderfuck, etc.). A precise taxonomy of trans identities is a tricky proposition, as there are new identities, and new directions for existing identities, being constantly articulated and negotiated. Each of these identities, as a different way of doing gender in a given time and place, will potentially have its own set of linguistic practices, ways of signaling the precise nature of the speaker's relationship to the socially reified categories of masculinity and femininity that are available in their community.

While this range of possibilities would present an interesting focus for study, for the purposes of this project, such a broadly defined range of gendered identities would actually confuse matters. If we are interested in the performances of socially-sanctioned masculinities and femininities, we would like all of our participants to have an investment in maintaining a consistent - and consistently recognisable - gendered presentation. For this project, then, we are restricting the range of trans identities to those people who have a fixed gendered identity (as either men or women), and who are fully committed to transitioning (i.e., to rejecting the gender role they were socialised into as children, and adopting the social role of their identified gender) (see chapter two for a more detailed discussion of the criteria for inclusion in this project).

By examining the linguistic practices of those people who have definitively crossed the boundary between socially-authorised categories that are popularly held to be mutually exclusive and exhaustive of the possibilities, we will be able to make some observations about the types of linguistic variable that seem accessible for this kind of deliberate gendered identity work. The fact of having crossed a category boundary marks these speakers as liminal, as people who have crossed a clearly demarcated line that has real-world consequences. This idea of liminality as a sociolinguistic window on salience draws on Rampton's notions of crossing (e.g., 1995; 1999) by further developing his framework into a useful tool for assessing salience through a careful analysis of the speech of people presenting an authentically gendered identity (unlike the inherently performative nature of crossing).

In principle, liminality should extend to other dimensions of identity as well for instance, ethnicity in heritage language communities - and if the approach adopted in this project proves a fruitful way to collect evidence about social drivers of language variation (as opposed to social effects of structural change) then future research may be able to develop a model of these social drivers. For this project, however, we will explore whether this approach is even feasible, through an examination of the gendered linguistic practices of trans people. 


\subsection{What this thesis aims to do}

So far, this chapter has laid the theoretical foundations for this project: it has introduced some of the key linguistic and social frameworks that inform the design and implementation of the research that follows, and it has drawn attention to the key areas of interest that this project hopes to address.

There are three broad research aims for this thesis. First, I want to examine the ways that gender is reflected in, and is created by, the linguistic practices of urban New Zealanders. To do this, I have collected a corpus of sociolinguistic interviews from Auckland that represents people who align with mainstream heterosexual identities as well as people in the more marginalised queer community. Second, I an interested in the effects of social change on the linguistic presentation of gendered identities. The participants included in the corpus form two distinct age groups which straddle a period of social change in New Zealand, and we will be able to compare the social effects of these changes with the linguistic systems of these two cohorts. Thirdly, I want to explore the analytical productivity of a liminality framework in variationist sociolinguistics, as an alternative to using perception studies to uncover which variables carry interpretable social meaning. The liminal identities included in this project are trans speakers, who have crossed the category boundary between masculinity and femininity, and whose experiences of gender make them particularly attuned to the gendered indexicality of various social cues.

Chapter two in this thesis will give some background on Auckland, the speech community sampled for this project, as well as providing some social and historical context, and introducing the Auckland Trans Corpus (ATC) as a linguistic resource. Chapter three will tackle some of the macro-methodology, such as the collection of interviews, the data handling processes, and the analytical approaches adopted.

The next three chapters will investigate individual variables. Chapter four looks at adjectival modification through intensifiers and moderators, which have shown robust gender differences in other varieties of English and which have not yet been examined from a variationist perspective in New Zealand English (NZE). Chapter five explores sibilants as a site for gendered linguistic practice, as a fronted /s/ in particular is associated with stereotypes of gay men and a particular style of femininity. Chapter six delves into the vowel space of NZE, which is known to be undergoing change and so presents an interesting test site for liminality, as the principles of sound change established through sociolinguistic and phonetic research would predict certain gendered patterns of variation as part of the structural change, regardless of the gendered indexicality of the variables. We will investigate the extent to which we can infer social meaning based on the articulatory practices of trans speakers.

In chapter seven, I will look at the commonalities and recurrent patterns observed across the three variables, and address the three broad research aims introduced 
above. In particular, I will discuss some of the generalisations about gender and linguistic variation in Auckland and the linguistic consequences of social change, and I will wrap up with some observations on liminality as an analytical tool for studying the social side of language variation. Chapter eight will provide some general conclusions on the project as a whole, and offer some directions for future research. 


\section{Chapter two: Community}

\subsection{Introduction}

For a project such as this one, aimed at exploring the links between gendered identity and linguistic performance, the speech community under examination has to meet certain criteria. The language variety spoken in the community should have been studied before, to provide a baseline for the variety overall and to act as a reference for the relative utility of adopting a multi-dimensional gendered space, and also to minimise the language documentation aspect of the project - see e.g., Meyerhoff (2015) for a discussion of the complexity of doing variationist work on under-documented languages. There must be a high enough population of queer and trans people to make the project viable, in terms of participant recruitment. The queer networks must be large enough and visible enough that queer exists as a recognised social identity in the broader community. And since the focus of this project is on gendered identity, it will also be necessary to recruit participants in such a way as to minimise the effects of other potential social factors on the variation observed.

The variety of English spoken in Auckland, New Zealand, has proven to be a good choice for this project. Generally speaking, New Zealand is a socially progressive society, where discrimination on the grounds of sexual orientation is prohibited, and many queer and trans people feel safe and supported. Auckland is the largest city in the country, with an estimated 1.6 million residents living in the greater Auckland region (out of a national population of approximately 4.7 million) (Statistics New Zealand, 2016), and acts as a service hub for business, education, and health care for much of the north island. It is also an ethnically diverse city, hosting a large proportion of the country's Maori, Pasifika, Asian, Middle Eastern, Latin American and African population, although the majority of the population of Auckland self-reports as being European/Pakeha ethnicity (Statistics New Zealand, 2016).

As a country, New Zealand has undergone substantial social changes over the past fifty years, including economic reforms, rapid urbanisation, a rise in immigration, and legal and social changes surrounding same-sex rights and citizenship (e.g., Wright 2013; Carlyon \& Morrow 2013; King 2003). In the wake of these changes, the social matrix of gender roles has also changed, and over a relatively short period of time: it is possible, then, to explore the linguistic correlates of some of these changes using a corpus with a relatively short time-depth. The purpose-collected corpus of sociolinguistic interviews used in this project includes straight, queer, and transsexual participants, and is organised into two broad age categories: those who grew up in a more traditionallyheteronormative culture (with a relatively clearly-demarcated division of gendered labour, and a generally hostile attitude towards queer people), and those who came of age 
in the period after the social upheavals of the late 1970s and, in particular, the Homosexual Law Reform debates of the early- and mid-1980s.

This chapter will provide some background on the social climate in New Zealand over this time period, and will also introduce the Auckland Trans Corpus: the methodology and rationale underlying the data collection, an overview of the participants included in this project, and some comments on the corpus as a whole.

\subsection{Social change(s) in New Zealand}

As a culture, New Zealand tends to see itself as egalitarian (e.g., King 2003: 507; Holmes, Marra \& Vine 2012: 1067; Duhamel \& Meyerhoff 2015), although of course there are differences that are nevertheless socially relevant - ethnicity, for example, or an urban/rural split. The extent to which this self-image is an accurate representation of the society as a whole is not entirely uncontested (e.g., Nolan 2007; Phillips 1996: 284), compounded in part by a seeming unwillingness to document or record evidence that contradicts this ethos historically, and spurred on by global financial changes in the 1980s and 1990s that saw a sharp rise in income disparity in New Zealand (Skilling 2013: 19). As well as these financial changes, the 1980s also saw a range of social causes take centre stage in New Zealand, including a strong anti-nuclear movement and the aftermath of the 1985 bombing by France of the Greenpeace flagship Rainbow Warrior, as well as the protests around the 1981 Springbok tour.

Alongside these largely political movements and changes, there were several other changes taking place in New Zealand society, two of which have direct relevance to this study. The first of these is a general shift in gender roles over several decades, away from the traditional masculinity of the rugged farmer-outdoorsman and the subordinate femininity of the supportive housewife, and towards something more urban and (theoretically) more equal. The second is the more specific move during the late $1970 \mathrm{~s}$ through to the mid-1980s to decriminalise homosexuality, which culminated in the 1986 passage of the Homosexual Law Reform Act, and which plays an important explanatory role for some of the variation that we have seen in this study.

\subsubsection{Changing gender roles: masculinity}

The traditional, idealised masculinity of Pakeha New Zealanders was "a giant of the backblocks - strong, resilient and modest, a man who could hold his own drink and enjoyed yarning with his mates, and who would eventually settle down as a loyal family man" (Phillips 1996: 267). This Kiwi bloke brand of masculinity, embodied by the likes of Edmund Hillary and returning war heroes, went largely unchallenged for several generations, until a gradual decline in militarism - tied to the end of conscription and compulsory training, and linked with protests against involvement in the Vietnam war in 
the 1970s - and economic shift eroded the foundations of this style of masculinity.

The rapid urbanisation and shift away from a rural economy meant an increased demand for technical skills and professional training, and Phillips (1996: 276) notes that the structures of traditional masculinity shifted without substantially changing: physical toughness as an ideal was replaced by business ruthlessness, and economic success became the measure of masculinity. Male culture continued to be centered around mateship, described by James \& Saville-Smith (1994) as a "celebration and reaffirmation of masculinity" (p.50) and valourising expressions of power: self-control, and control over others. It wasn't until the mid-1990s that this began to destabilise (Phillips 1996: 277), partly because of a harsh and sustained critique of bloke culture from feminism, and partly because new styles of masculinity - including gay men and international sophisticates - became more visible in urban New Zealand.

There is a marked generational split in my interviews with men, between the older men who grew up steeped in an urbanised traditional masculinity, and the younger men who grew up under a post-1990s contemporary masculinity. The older men see femininity and masculinity as two distinct cultures, and the points of contact between these two cultures are fraught with tension. For older straight men in particular, the culture of women is one of nurturing and fragility that must be handled with great care, while that of men is more characterised by bluntness and a hierarchical structure. These men position themselves as generalist experts, and seem frustrated that women - and young women in particular - are unwilling to accept their authority unquestioningly. The men-women difference is arguably less conflict-prone for older queer men, because unlike their straight counterparts, they do not particularly expect women to figure prominently in their lives, either emotionally, sexually, or domestically. For them, women are a world apart, and while some resent the intrusion of lesbians into formerly men-only groups and spaces, for the most part they expend very little energy on the project of women.

For the younger men that I interviewed, gender seems to represent much less of a cultural divide. Both queer and straight men interact socially with women on a regular basis, and count women among their close friends. Certainly there is a sexual dimension to these interactions for straight men that is notably absent for queer men, but even this dynamic seems less gendered among the younger cohort. Older men spoke about holding doors open and paying for drinks and dinner, with one man telling me that he was teaching his son to continue this tradition, even though some women might not like it, because he saw it as an important part of being a man. Younger men seemed to adopt a less chivalrous attitude towards women, more in line with equals hanging out, and also seemed less concerned with hierarchy among their peer groups of men.

Of course, it should be noted that the younger straight men included in this project are generally middle class and highly educated (as are the older men), so they may not be generalisable to all New Zealand, or indeed to all Auckland, men in their age 
bracket. However, since their linguistic data is what we will be basing our subsequent analyses on, the social dynamics and social positioning of these particular young men are relevant to this project. We will simply have to bear these demographic limitations in mind when we are trying to generalise to the broader population.

\subsubsection{Changing gender roles: femininity}

The traditional role of women in New Zealand, or at least in those parts of New Zealand dominated by colonial British influence, is that of wives and home-makers: responsible for raising children, cooking, cleaning, and doing various domestic chores on a working farm. In the rural, farm-based economy of the not-so-distant past, these skills were valued in a way that they are not now (e.g., James \& Saville-Smith 1994: 54-56). Women contributed directly to the running of the farm, and therefore to the financial success of the family. Education and career options were slim for women under this model: two of the older queer women I interviewed talked about deciding quite young that they would pursue careers in teaching and nursing, not out of any particular interest in those fields but because they were the only viable alternatives to getting married and running a farm.

The gradual economic shift that eroded the feasibility of small-scale farming and led to rapid urbanisation in New Zealand, and the subsequent shift towards a valourisation of education and careers in management, should by rights have presented women with new opportunities. However, there was still an expectation that women would be home-makers, and would support their husbands and raise their children. This new economy equated value with money earned, leaving domestic women at a distinct disadvantage: with their skills unpaid and largely under-appreciated, their lives were dependent, relegated to supporting their partner rather than pursuing their own ambitions.

Second-wave feminism came to New Zealand in the early 1970s (Macdonald 1993: 161), characterised by sporadic protests and consciousness-raising groups (Kedgley 1985), and a critique of the gender hierarchy in New Zealand. More women began to enter the workforce, albeit with lower pay and a disproportionate representation in certain low-paid and poorly-regarded fields (Phillips 1996: 274), a bias that continues today (Hyman 2004; Ministry for Women 2016). However, the younger women interviewed for this project (both straight and queer-identified) feel positive about their prospects. They report seeing increasing numbers of women in science and technology programmes, they do not feel that they are pressured away from particular fields, and they believe that they have the freedom to pursue whatever appeals to them. While they may yet encounter barriers in professional, political, and economic spheres, they generally see a future with more possibility and greater gender equality than their mothers faced. This sentiment is echoed by some of my older women participants, who are perhaps more cautious in making claims about gender equality but who nevertheless agree that younger women have more freedom than they themselves did. 


\subsubsection{The Homosexual Law Reform bill}

Although the history of (il)legality of male homosexuality in New Zealand stretches back into the mid 1800s, it wasn't until the 1960s that any kind of organised law reform movement got started (Laurie \& Evans 2009), and it wasn't until the 1980s that the movement began to gain any ground (Laurie \& Evans 2009; Evans, Laurie \& Instone 2009). The particular chronology of the law reform, and the specific cultures and social mores at play at that time, are complex and contested by the various parties involved. For the lesbians and the gay men involved in the movement, it was a political issue of equality and social justice (e.g., Laurie \& Evans 2009a; also various participants in the ATC): their public involvement in the movement put not only their professional lives on the line, but often their personal and family lives were also at stake, as their involvement brought them into direct conflict with religious organisations, their friends and neighbours, and the police.

The movement itself also had internal tensions and divisions, often about the particular issues that should be focused on, and the approaches that should be taken. From the perspective of some of the people involved in the movement, the investment of time and money by US Evangelical Christian organisations in the anti-law reform campaign was a turning point with regards to public opinion in New Zealand: some of the ATC participants felt that many New Zealanders resented the interference from abroad. From the perspective of some of the gay men not involved in the campaign, the turning point was fundamentally a more pragmatic one: when the law reform issue was framed in terms of the AIDS crisis, then the question of decriminalisation became one of public health rather than morality, and pragmatism won out (Parkinson 2009; Simpson 2016).

These conflicting accounts of what actually swung public opinion in favour of the Homosexual Law Reform Bill demonstrate the extent to which the period of public debate on decriminalisation was a tumultuous time for lesbians and gay men in New Zealand, and for society in New Zealand more generally. The bill was passed in Parliament on July $9^{\text {th }} 1986$, signed into law as the Homosexual Law Reform Act on July $11^{\text {th }}$, and came into effect on August $8^{\text {th }}$. The fact that life continued more or less the same as it always had - contrary to the worst-case-scenarios passionately argued by those opposed to the bill - is thought by some (e.g., Simpson 2016) to explain the relative ease with which the Civil Union Act 2004 was passed, as well as the subsequent Marriage (Definition of Marriage) Amendment Act 2013, which defined marriage as "the union of 2 people, regardless of their sex, sexual orientation, or gender identity" (Marriage (Definition of Marriage) Amendment Act 2013, section 5). When the Marriage Amendment Act was passed, MP Maurice Williamson (member of the right-of-centre New Zealand National Party) gave a widely-publicised speech in favour of the amendment (Williamson 2013) in which he acknowledged voices of dissent in his constituency while plainly arguing against their fear-mongering and scare tactics in favour of equality. This speech in many ways shows the degree to which New Zealand 
broadly has come to accept the mainstreaming of gay and lesbian identities in the 30 years since the initial Homosexual Law Reform Act was passed.

What is interesting for this study is the effect that this culture shift has had on queer people in New Zealand. The older queer participants in the ATC came of age, and discovered their sexuality, in a social climate that was openly hostile towards them. They recall police raids on clubs in Auckland and in Wellington, and the long shadow cast by the 1964 murder of Charles Aberhart in Christchurch - not only the violent killing itself, but the acquittal of the six youths charged in the case and the general societal indifference to what had happened. Simpson (2016) reports that many of his older gay male participants still react with fear to the police, decades later. For people in this age bracket, community offered not only a source of safety and solidarity, it was also the central hub in a network of friendship, love, and family. This is not to say that it was unproblematic - publicly known bars and cruising spots were ripe for police intimidation and gay bashings, and there was some strong antagonism between certain groups of lesbians and certain groups of gay men (some of which still exert a force in mixed groups of older queer people (Bird 2015)). But becoming a member of a lesbian and/or gay community was an important part of discovering and fully embracing their identities outside of the mainstream.

In contrast, the younger queer participants in the ATC generally do not report the same degree of engagement with the queer community, in part because they do not face the same degree of social persecution as their older counterparts. This is not to say that individuals do not face barriers to acceptance, happiness, and general support; studies in New Zealand and abroad continue to find that LGBTQ youth are disproportionately represented in statistics on depression, suicide, homelessness, unemployment and substance abuse, and these statistics tend to be intersect with indices of ethnicity and socioeconomic status. However, at least within the ATC, which is broadly ethnically homogenous and economically middle-class, most of the younger participants report having a generally supportive environment, and having never experienced overt discrimination at work or at school as a result of their sexuality. Their friendship groups tend to align with other aspects of their identity: interest in sports, or in board games, or through networks rooted in school or employment. For these participants, their sexuality is one aspect of their identity (often a relatively minor one), and does not in and of itself carry political overtones; for older participants, identifying as gay or lesbian remains a political act. For the younger queer participants in the ATC who were recruited through a queer organisation (either a campus-based university group or a community choir), this organisation was the only overtly queer group they reported being part of; for those recruited through other methods (e.g., snowball sampling), they did not report being involved in overtly queer groups at all. In contrast, the majority of the older queer participants were involved in several queer organisation, including business associations, sports groups, and social clubs. 
There is also a dimension of gendered difference between these two age groups. Among older people, there are gendered tensions that seem absent between the younger cohort: older lesbians are warier of gay men than are younger queer women, and older gay men seem more hostile towards lesbians than younger queer men.

Younger queer people in urban New Zealand have had a very different experience of queerness than their older counterparts, and this is affects not only in their orientation to personal identity as a political and social statement, but also to the role of community in their lives at all. This is reflected in the differences in labels that queer people use to define their identities. Younger people seem to look for the most precise individual terms to identify themselves (e.g., pan-romantic demi-sexual), while older people tend to prefer macro-labels (e.g., lesbian) that draw clear lines of association between themselves and others. On one level, creating communities is about making a distinction between $u s$ and them, which has arguably characterised the experiences of the older speakers when they were younger. Younger speakers seem oriented towards individualism instead, never really having been in the position of being othered in the same way as their elders.

These changes in social attitude towards queer identities does not only affect queer people themselves; they are part and parcel of a larger re-ordering of social categories, particularly masculinity. As discussed above, the changing profile of the Kiwi bloke began to license men who were educated and urbane, qualities previously branded as effeminate and suspect; with a wider acceptance of gay men as part of the social fabric of New Zealand, the younger straight men in the ATC - who came of age post-HLR - do not feel as strong a need to distance themselves from queer male identities. This is not to say that their heterosexuality is negotiable, but rather that when they are perceived as gay or bisexual, it is an error but not a face-threatening miscategorisation.

\subsection{The Auckland Trans Corpus (ATC)}

With many of the major social changes in New Zealand occurring over a relatively short time period, it is possible to split the population into two groups, one acculturated to the older, more traditional social matrix, and one to the more contemporary one. This is the basic structure of the Auckland Trans Corpus (ATC), the body of sociolinguistic interviews that was collected in Auckland between 2013 and 2015, which forms the basis for this project. It has been constructed with representative straight, queer, and transsexual speakers of English in Auckland, and recruited participants via snowball sampling techniques, supplemented by an overt appeal for participants. This section describes the rationale behind the design of the corpus, and introduces the participants. 


\subsubsection{Designing the corpus}

Because the aim of this project is to explore socially-constructed gendered identities, it makes sense to use socially-motivated definitions of gender and sexuality. What I mean by that is to categorise people based on how they enact their identity socially, as much as by how they self-identify. It is not enough, for example, for a man to be sexually attracted to men for him to be classified as queer for the purposes this project: who he is physically involved with will have less impact on his linguistic choices than the social groups that he is part of. If he is a member of queer social networks, and considers his sexuality to be an important part of who he is, then we may expect to find some influence of community-level norms on his behaviour. This is in contrast to MSMs (Men who have Sex with Men), an alternative category for men who participate in same-sex sexual encounters, but who do not position themselves (or allow themselves to be positioned by others) as part of any gay, LGBT, or queer community, and who do not identify with any aspects of queer culture. As my primary interest is in the different social enactments of masculinities and femininities, MSMs are not particularly interesting, except potentially as enactors of a fairly staid heteronormative masculinity. Until this is empirically tested (which would be an interesting study, but not part of this one), it is methodologically sounder to look to the speech of strictly heterosexual men for examples of heteronormative masculinity.

I have chosen to entangle the distinct but related dimensions of gender (masculine/feminine) and sexuality (queer/straight). This is partly because I believe that there are observable differences between the social realisation of queer and straight masculinity (and also between queer and straight femininity, although it is often harder to pinpoint what those differences are or might be); this is also because sexuality is inherently gendered. To be a lesbian is to necessarily be a woman: attraction to women is not enough, because that would classify straight men as lesbians as well. Sexual orientation and gender are not the same, but they are not entirely separable either, and to attempt to separate them is to do a disservice both dimensions.

Participants, then, were classed as being either queer or straight men or women, based on the combination of how they self-identified, and the social networks they reported participating in. Trans participants were classed as trans if they had been in transition for at least one year at the time of recording; people who did not identify with either masculinity or femininity in some way, or who were not committed to fully transitioning (e.g., people identifying as genderqueer, genderfluid, polygender, etc.), were not included in the following analyses. The potential fluidity of their gendered linguistic practice may make for fascinating research in the future, once the groundwork has been laid by a study such as this one.

It is important to note that these parameters were put in place not as means of policing how people identify or reifying some identities as more authentic than others; rather, the aim was to group speakers into units who can reasonably be assumed to be 
pursuing some kind of coherent gendered identity. Of course, there is scope for individual variation within any of these group categories: queer man is not a monolithic identity, any more than straight woman is. But within these categories, we can attempt to group people by objective measures of similarity (e.g., age, ethnicity, level of education, etc.), and then recognise that, inasmuch as we make generalisations over groups of people, those groups are composed of individuals, so the abstractions that we make are abstractions over disparate aggregates of unique individuals.

The participants in the ATC are overwhelmingly of NZ European/Pakeha descent, and all have pursued or are pursuing some form of tertiary education. This homogeneity was not the intention at the outset of the project: this project could have explored Maori and Pasifika performances of gender as a point of comparison between the masculinities and femininities imported through colonisation and those more indigenous to the South Pacific. However, the social and community networks that I was able to access were ethnically very homogenous and dominated by NZ Europeans/Pakeha, so that has determined the range of analysis possible. The exception to this trend is the two younger trans women included in this project, both of whom identify as Pasifika, and for whom their Pasifika heritage is an important component of their identity and their community lives; they are discussed in greater detail in $\$ 2.3 .2 .3$, below.

Ideally, of course, we would like all of our participants to be local, born and raised in Auckland, and never having spent any significant amount of time outside the region. However, the demographic reality of Auckland, and of New Zealand more generally, makes this difficult to achieve. Many people move to Auckland from more rural areas, for a mix of educational, employment, cultural and social reasons, and it is not uncommon for New Zealanders (particularly younger ones) to be well-travelled internationally. Restricting the pool of participants to native Aucklanders would have made data collection a much longer and more complicated endeavour, and may not even have been possible in the case of trans participants, as many trans people from around the North Island move to Auckland to access a wider range of services than is available outside of the city. Nevertheless, I wanted some degree of control over potential regional variation, so the following compromises were decided on, in an attempt to ensure that language represented in the corpus approximates Auckland English:

1. For trans speakers, they must have started their transition (and continued it) in Auckland, so that the gendered norms that they are adopting are those modeled by Aucklanders more generally.

2. For queer speakers, their connections with queer communities and queer organisations must be centred in Auckland, so that the community norms they are orienting to are local.

3. For straight speakers, they must have lived in Auckland for most of their lives, and consider Auckland to be home. 


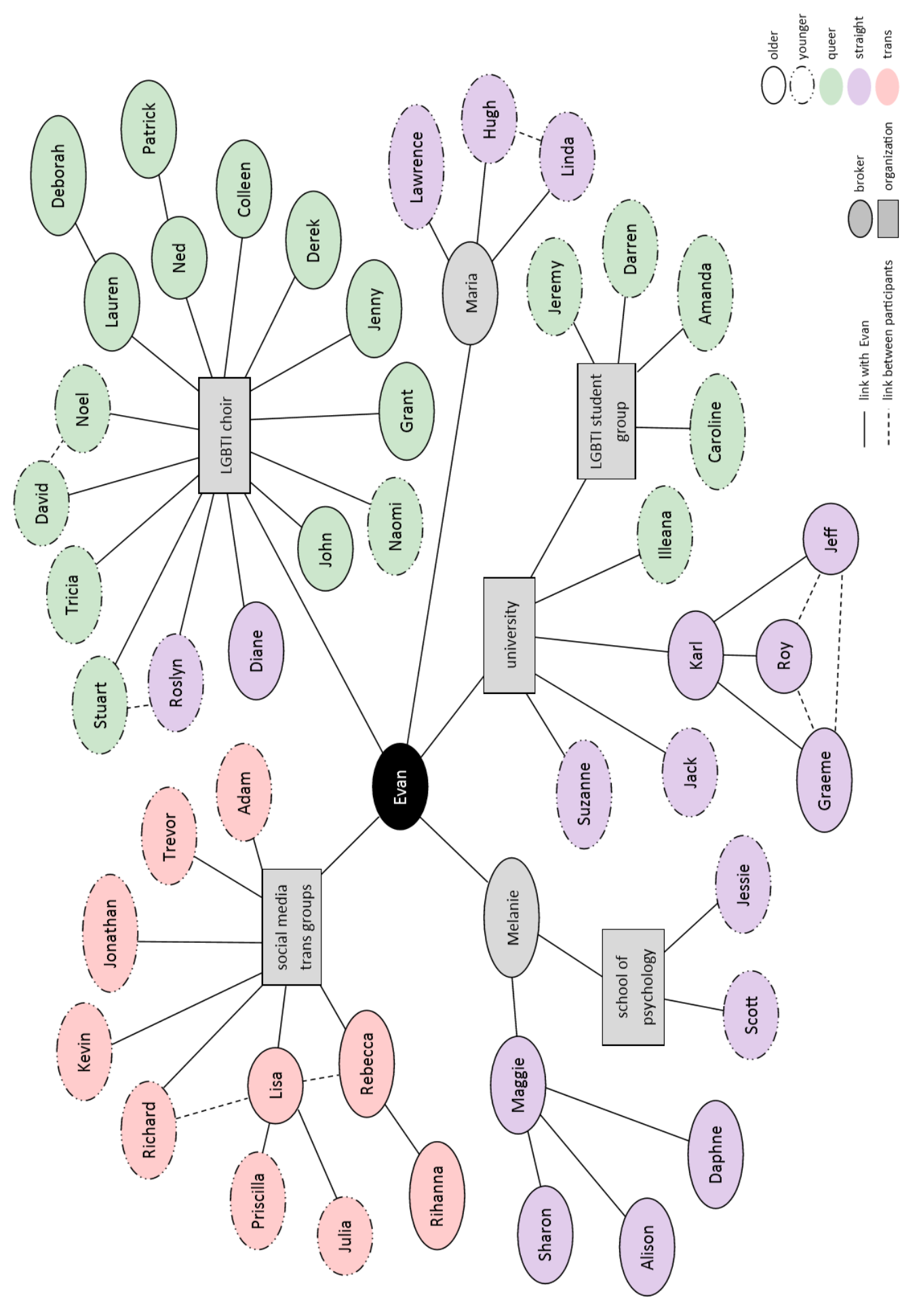

Figure 2.1: Network diagram of participants in the ATC 
Although these compromises are not perfect - number 3 in particular leaves quite a lot of freedom for people to have come from elsewhere, and potentially to have imported regional dialectal features in their language - but with a city so densely populated by migrants and immigrants, this approach arguably produces a sample of speakers that actually is representative of the English spoken in Auckland, regardless of whether it is (in the narrowest sense) Auckland English.

\subsubsection{ATC participants}

There were two primary approaches I used to recruit participants. One was through snowball sampling, where participants recommended people from within their own social networks; and the second was to get in touch with people through informal or casual organisations, often online - this proved particularly useful for recruiting trans participants, as much of the community-building work that goes on is conducted through social media. These approaches were supplemented as necessary through direct appeals for volunteers, via a postering campaign on campus at one of the universities in Auckland.

Figure 2.1 provides a visual representation of the relationships between participants, and the routes by which I made contact with them. Straight participants are shown in purple, queer participants in green, and trans participants in pink (note that all names used here and throughout the thesis are pseudonyms). The age classification of the participants is indicated by the outline on their name bubble: younger speakers are shown with dashed borders, and older speakers with solid borders. Names in grey represent brokers: people who were not themselves participants, but who facilitated introductions with participants or with organisations. Organisations are shown in grey boxes. Solid lines between participants or organisations indicates the route by which they are connected to me: for example, Deborah was introduced to me by her partner Lauren, who I met through the LGBTI choir (see \$2.3.2.2 for more detail). Dotted lines indicate strong ties between participants, independent of how they are connected to me. For example, David and Noel - both of whom were recruited through the choir - are partners; Stuart and Roslyn are flatmates and close friends. These lines do not represent the only links between participants - to attempt to diagram the entire complexity of friendships would render the chart uninterpretable - but they show the major multiplex links that give an indication of strong ties between participants.

More information on recruitment and participants is given in the following three sections.

\subsubsection{Straight participants}

The straight participants in this study are taken to be representative of the unmarked default categories of masculinity and femininity. There are four categories: older straight women, older straight men, younger straight women, and younger straight men; a 


\begin{tabular}{|c|c|c|c|}
\hline & Participant & Age & Occupation/other notes \\
\hline older & Diane & 60 & psychotherapist \\
\hline straight & Maggie & 54 & art restoration \\
\hline women & Sharon & 53 & teacher, freelance writer \\
\hline \multirow[t]{2}{*}{$(o s w)$} & Alison & 53 & librarian \\
\hline & Daphne & 51 & creative industries (advertising, film) \\
\hline \multirow{4}{*}{$\begin{array}{l}\text { older } \\
\text { straight men } \\
\text { (osm) }\end{array}$} & Karl & 54 & university lecturer \\
\hline & Jeff & 52 & mining industry executive \\
\hline & Graeme & 55 & managing director (import business) \\
\hline & Roy & 51 & self-employed (public transport consultancy) \\
\hline \multirow{5}{*}{$\begin{array}{c}\text { younger } \\
\text { straight } \\
\text { women } \\
\text { (ysw) }\end{array}$} & Jessie & 25 & postgraduate student \\
\hline & Caroline & 25 & undergraduate student \\
\hline & Roslyn & 32 & waitress, undergraduate student \\
\hline & Linda & 30 & ESL teacher \\
\hline & Suzanne & 22 & postgraduate student \\
\hline \multirow{4}{*}{$\begin{array}{c}\text { younger } \\
\text { straight men } \\
\text { (ysm) }\end{array}$} & Hugh & 35 & architectural designer \\
\hline & Jack & 30 & postgraduate student \\
\hline & Scott & 24 & research assistant \\
\hline & Lawrence & 21 & undergraduate student \\
\hline
\end{tabular}

summary of their demographics is presented in Table 2.1. Because there are no 'straight communities' akin to those found with queer and trans groups, my recruitment relied heavily on two brokers (Maria and Melanie), and on connections with one of the universities in Auckland.

Maria, one of the brokers in Figure 2.1, is a South American immigrant to New Zealand, and has extensive connections in Auckland, through her own work and that of her partner. She arranged for Hugh and Linda, a married couple with a young child, and Lawrence, to participate in the project. Melanie is the other broker who proved instrumental in recruiting straight participants: she is a native New Zealander who has lived abroad, and also has extensive social contacts through both her own and her husband's networks. She introduced me to Maggie, who made connections for me with her friendship network, so the three participants shown in Figure 2.1 branching off Maggie are casual acquaintances of each other, although they are not a particularly tight friendship group.

Melanie also negotiated contact for me through the school of psychology at one of the universities in Auckland, through which I recruited Scott and Jessie. I also put recruitment posters up around this same university, and recruited Jack and Suzanne through a direct appeal for volunteers. Karl is also affiliated with this university, and he put me contact with his network friends; these four men have been close for well over a decade, and have been involved in several sports teams together during that time. They socialise as a group regularly.

It should be noted that, although Roslyn and Diane are members of the LGBTI choir, both identify as straight and report the choir as their only meaningful connection 


\begin{tabular}{|c|c|c|c|}
\hline & Participant & Age & Occupation/other notes \\
\hline \multirow{4}{*}{$\begin{array}{c}\text { older queer } \\
\text { women } \\
\text { (oqw) }\end{array}$} & Lauren & 54 & doctor \\
\hline & Deborah & 58 & teaching assistant \\
\hline & Jenny & 59 & nurse \\
\hline & Colleen & 61 & teacher, librarian \\
\hline \multirow{4}{*}{$\begin{array}{c}\text { older queer } \\
\text { men } \\
\text { (oqm) }\end{array}$} & John & 50 & logistics, technical work (telecom company) \\
\hline & Grant & 65 & self-employed (musical instrument repair) \\
\hline & Ned & 53 & teacher \\
\hline & Patrick & 57 & financial planner \\
\hline \multirow{4}{*}{$\begin{array}{c}\text { younger } \\
\text { queer } \\
\text { women } \\
\text { (yqw) }\end{array}$} & Amanda & 22 & undergraduate student \\
\hline & Tricia & 25 & postgraduate student \\
\hline & Naomi & 26 & engineer, project coordinator \\
\hline & Illeana & 23 & parliamentary executive assistant \\
\hline \multirow{5}{*}{$\begin{array}{c}\text { younger } \\
\text { queer men } \\
\text { (oqm) }\end{array}$} & Darren & 24 & undergraduate student, sauna manager \\
\hline & Noel & 21 & software developer \\
\hline & David & 21 & software developer \\
\hline & Stuart & 25 & embassy consular officer \\
\hline & Jeremy & 26 & postgraduate student \\
\hline
\end{tabular}

to, or interaction, with the queer community. Diane joined the choir several years ago when her sister was a member, although her sister has since left Auckland; Roslyn joined through her close friendship with Stuart.

\subsubsection{Queer participants}

If the straight participants are considered representative of mainstream, heteronormative Auckland English, then the queer speakers present an alternative set of femininities and masculinities, one less invested in the traditional norms. There is of course considerable scope for variation under a necessarily broad umbrella such as queer, so some attempt was made to control for various demographic factors in selecting queer participants for this project. Age is a known - or strongly suspected - relevant social factor, so participants were chosen who can be grouped into two age brackets, reflecting social change in New Zealand. Ethnicity was also controlled for, although less by design and more by social reality (see below), and perhaps as a corollary of ethnicity, education and socioeconomic status were also relatively controlled. Additionally - and again, not by design but rather by happy accident - the queer participants included in this project seemed largely invested in presenting and maintaining relatively coherent social identities, within their age and gender groups. That is to say, older queer men shared much in common stylistically and socially, as did the younger queer men, although there were differences between the two age groups; similarly, the younger queer women seemed to orient to a broadly similar idea of young queer femininity, and the older queer women likewise seemed to have converged on similar styles. This is not to say that there is no difference between individuals, because that is clearly not the case - there are always differences between people. Rather, the people comprising these four gendered 
identity groups seem largely to orient internally towards similar styles of masculinity and femininity, so there is a strong likelihood that they are also converging on similar linguistic styles.

The queer participants were largely recruited through two organisations: a community-based choir, and a loose network organised through one of the universities. Table 2.2 provides a summary of the queer participants, organised by age and gender.

The choir in question has been active in the queer community in Auckland for several years. It is a mixed-gender choir with no auditions, and incorporates social elements in the form of a standing dinner invitation before each rehearsal and a visit to a neighbourhood bar afterwards, as well as member-organised parties at various points through the season. The choir's emphasis has traditionally been on community, although the focus on performance has been increasing gradually with successive choir directors. Support for the choir in terms of concert attendance typically comes from within the queer community and the friendship and family networks of the members, and the choir is involved with both a national organisation of choral music in New Zealand, and with several other queer organisations in Auckland.

The membership of the choir is mostly older people, and almost exclusively NZ European/Pakeha (plus immigrants, mainly from Europe and Canada). In the past, the choir was more mixed, but there was a schism several years before I became involved, which resulted in the Maori and Pasifika members leaving en masse. I joined the choir at the start of the 2013 season, and spent around six months getting to know people - and letting them get to know me - before making any approaches to participants. As part of my involvement with the choir, I led a workshop on basic phonetics and the IPA for interested choir members, after which the musical director (unknown to me, and neither asked for nor expected) advertised my project to the membership. This certainly helped increase the number of recruits from the choir, particularly among the older members; it is interesting to note that, for older choir members, there seemed to a be a generally higher investment in community-building than among the younger ones; they were more willing to make themselves available to support my research, and to help me recruit from their other networks.

The LGBTI student group that served as a secondary recruitment point for queer participants, and which proved a valuable resource for recruiting younger queer people in particular, is a loosely organised local chapter of a national organisation for queer tertiary students. They maintain an office/drop-in space on campus, but their principal regular event is a weekly social held at a nearby café, and a lot of what they do happens online. I joined the group and attended a few meetings and get-togethers, but the group members who volunteered to participate were all online contacts, rather than people I met at any of the official events. Illeana, the sole queer participant not contacted through one of these two groups, contacted me in response to my postering drive; she is not affiliated with the student group due to political differences, but she is involved in queer and queer-positive 


\begin{tabular}{clcl}
\hline Table 2.3: & Trans participants in the ATC \\
\hline & Participant & Age & Occupation/other notes \\
\hline $\begin{array}{c}\text { older trans } \\
\text { women }\end{array}$ & Rebecca & 58 & unemployed, self-published author \\
(otw) & Rihanna & 46 & office manager \\
\hline $\begin{array}{c}\text { younger } \\
\text { trans women }\end{array}$ & Priscilla & 38 & manager (social services) \\
(ytw) & Julie & 20 & creative industries (writing and performance) \\
\hline younger & Richard & 26 & digital journalist \\
trans men & Kevin & 20 & unemployed \\
(ytm) & Erich & 30 & journalist \\
& Jonathan & 32 & logistics (electronics company) \\
& Adam & 32 & unemployed \\
\hline
\end{tabular}

organisations elsewhere.

\subsubsection{Trans speakers}

With straight and queer speakers presenting alternative - and potentially opposing styles of masculinity and femininity, the trans participants offer us an insight into the social salience of linguistic choice. They are systematic and careful observers of gendered behaviour, and have made a deliberate decision to commit to transitioning. To effect this, they use all of the resources available to them, including language. Other resources, such as hormonal and surgical interventions, vary from speaker to speaker, limited in large part by financial accessibility and health care support availability; but language and linguistic presentation are resources that are immediately available, and some of the trans men in the ATC report that language was particularly important to their masculine presentation early in their transition, when they felt that their physical appearance was ambiguous and that people were relying on secondary cues to assess their gender. Whether this is objectively true - that is to say, whether people were assessing their language use - has not been verified, of course, but the fact that these trans men felt that it was meant that they saw language as a useful resource, and used it strategically. My previous work with trans men in Ottawa, Canada (Hazenberg 2012), echoes this notion: as physical appearance started to align with societal expectations (e.g., growing facial hair, chest masculinisation), these participants reported relying less on performances of stereotypical masculinity, and drifted towards a more queer-aligned linguistic practice. Although none of the trans men in the ATC stated this relationship quite so explicitly, they did report being more comfortable being read as queer once they were confident that they were read as men: when an androgynous physical appearance raises the possibility of being misgendered, they are more likely to avoid ambiguity in other modes of presentation (including linguistic ones).

As with the queer participants, I spent approximately six months getting to know the trans community before approaching anyone as potential recruits. In Auckland, and indeed across most of New Zealand, much of the networking between trans people is 
conducted online: Facebook is the main platform that they rely on, largely because it allows the creation of closed and invisible groups, which provides a measure of safety and protection for people who are just starting to come out, or who are stealth (i.e., who live their lives with no public disclosure of their identity as transsexuals), or who simply want to compartmentalise the different facets of their lives. There are least ten fairly stable (i.e., they have been in existence for a few years) secret Facebook groups for trans men in New Zealand, and membership in these groups is arranged by a known member of the group vouching for the new applicant. Table 2.3 provides a summary of the trans participants included in this project.

The trans community in New Zealand is, on the whole, very gendered: trans men don't have much to do with trans women, either online or in person, and vice versa. This is likely due in part to the different needs of the two groups, as many of the groups deal with frequent requests for advice on dealing with the public health system, and are quite specific. There are also tensions around the question of authenticity and experience, particularly on the part of older trans women who often position themselves as gatekeepers: as people transition at younger and younger ages, and as the range of services available to them increases, some older trans women use increasingly narrow definitions of transsexuality and transition to discredit the experiences of younger trans people, particularly trans men. Interestingly, this attitude has also begun to show up in some of the trans men's Facebook groups, in the form of questioning the validity of people who identity as genderqueer, or who use other non-binary terms. There is also some overt misogyny and misandry in circulation in some of the Facebook groups, although these groups tend to dissolve quite quickly (and bitterly), and new groups emerge to replace them.

These disagreements and conflicts can spill over into the real world, too; in a small community in a small country, everyone knows everyone else. The diagram in Figure 2.1 does not show all of the multiplex ties between the trans participants, because they all know each other through multiple channels; the two secondary links that are shown represent major ties between participants. Richard and Lisa are very good friends and have supported each other through several personal and familial crises. In contrast, Lisa and Rebecca are openly hostile towards each other, in a way that has resulted in very public arguments played out between them, and that has resulted in a very bifurcated community of trans women. This schism is partly responsible for the paucity of trans women in the ATC: Rebecca was a very early volunteer, who subsequently vouched for me as a non-predatory researcher in various online forums and communities, and her endorsement turned several other trans women away from the project.

Lisa was eventually able to help introduce me to Julia and Priscilla, both of whom are Pasifika women from South Auckland. This difference in ethnicity complicates the analyses that follow, in that South Auckland English is perceived as qualitatively different from the majority Auckland English: it is seen as being heavily 
influenced by Pasifika and Maori cultures, and the suburb itself is thought of as a tough neighbourhood with a 'gangsta' attitude and bad or rough language (Duhamel \& Meyerhoff 2015: 243). In fact, South Auckland English has not been extensively studied from a sociolinguistic perspective, so the extent of quantitative difference between it and general Auckland English is unknown. Ideally, then, we would elect not to include participants in a study such as this one who may very well not be orienting to the same set of language norms as the majority of the participants, but because we have no other younger trans women in the ATC, excluding them is in fact excluding any representation of younger trans women whatsoever. In fact, there are some interesting patterns that will begin to emerge when we turn our attention to the individual variables, which point to some interesting indexical links between South Auckland's perceived toughness and young masculinity; this will be touched on in the variable analyses that follow, and explored in more depth in section $\$ 7.4 .4$.

Finally, it needs to be noted that there are no older trans men in the ATC. There are two ready explanations for this, although the truth is likely to be partly both. First, it is possible that there are no older trans men in New Zealand, given the comparatively late emergence globally of trans men as an identity and as a bodily practice. Certainly there have been 'women' who have adopted the role of 'men' throughout history, but as a visible practice rooted in a community of shared experience, trans men did not become a phenomenon in North America until the 1970s and 1980s (Stryker 2008: 114). The second possibility is that there are older trans men out there, but that they are not connected to any of the (largely computer-mediated) communities and networks that I have had access to. Certainly no one I spoke with knew of any older trans men in the Auckland area; I have interacted online with two trans men who are over 40, but they were not yet one year into their transition at the time I was collecting interviews, so they did not meet the minimum criteria I had established to promote a coherent gendered linguistic practice. Neither one lived in Auckland, either: one lived further north, and one lived outside of Christchurch.

The history of transsexuality in New Zealand is hard to trace, as it is everywhere, partly because much of the development of the culture happened in the undocumented margins of society. However, it seems unlikely that there was much social or institutional support for trans men in New Zealand earlier than in North America, so it seems reasonable to think that any trans-masculine people who may have been around for decades are not well-integrated into contemporary communities. Also, some of the older queer women that I have gotten to know in New Zealand through this project lament the loss of the butch dyke identity in New Zealand: they feel that many of the younger queer people they meet, who in the past would have lived as butch women, are opting instead to transition to men. This suggests that, indeed, the FtM identity is a relatively recent one, compared to the corresponding MtF identity. 


\subsubsection{The ATC as a linguistic resource}

All sociolinguistic corpora have their weaknesses, and the Auckland Trans Corpus is no exception: the uneven numbers of trans speakers makes a very detailed study of the linguistic practices of trans people impractical, and the fact that not all of the speakers were born in Auckland make it hard to make generalisations about Auckland English as a variety of English. However, it is nevertheless a useful and unique linguistic resource, both for this particular project, and for studies of New Zealand English more broadly.

The deliberate focus on speakers orienting to potentially different masculinities and femininities allows for a systematic examination of the linguistic practices of these different gendered identities in a way that is not possible with a more traditional straightforward binary breakdown of gender. The inclusion of trans speakers also means that we are able to investigate the differences between linguistic variation that correlates with gender statistically (which may be a byproduct of structural changes underway in the linguistic system more generally), and variation that seems to actually carry some degree of accessible gendered indexicality. The two age groups, while certainly not representative of the entire demographic range of Auckland, reflect two broadly different sets of social expectations with respect to gender and sexuality, and can shed light on the role that large-scale social change plays in socially-motivated language change.

This project is not primarily interested in the documentation of New Zealand English at a more structural or phonetic level, although the ATC will be a valuable resource for future studies in these areas. This project, for example, is not making use of the read passage component of the interviews, as an analysis of style shifting within the larger question of gendered identity would have added another substantial layer of analysis to an already complex study; this has been set aside for future research. The ATC offers a recent corpus of English from a major urban centre not previously examined in New Zealand, and can contribute data to ongoing phonetic and morphosyntactic changes, as well as investigations into regional differences across the country. The fact that several of the speakers have come from different regions in New Zealand can also provide an interesting point of comparison with other corpora of Auckland English, currently under construction or not yet undertaken: is Auckland English distinctive enough as a variety that it can only be studied with native Aucklanders, or are the linguistic practices of the ATC participants who originally came from elsewhere comparable to natives? And, at a more theoretical level, if a substantial portion of the population of Auckland is originally from somewhere else, how representative of the English spoken in Auckland is a corpus that includes only born-andraised Aucklanders?

Finally, although discourse analysis is not part of this project, the ATC provides a rich source of data for explorations on a larger scale than the variationist and sociophonetic analyses in this thesis. In particular, the data is well-situated for 
investigations of: narrative structure, with the large number of topic-comparable stories (e.g., adventures abroad); differences in emotional affect and humour, with several of the participants providing a combination of sad/touching stories and quite funny ones; and the role of international travel experience in creating a national New Zealand identity. The metalinguistic and metapragmatic observations on language and gender will also be a good resource for language and gender investigation in the future. 


\section{Chapter three: Methods}

\subsection{Introduction}

The previous chapter described the composition of the Auckland Trans Corpus (ATC), and why it is structured the way it is. As a linguistic resource, the ATC provides a good source of conversational data for investigations of both gendered identity in language variation, and of New Zealand English as a distinct variety. However, there is a difference between an interview as a social object and as a workable data set, and this chapter will provide a broad introduction to the methodologies and analytical tools used to bridge this gap.

It should be noted that this project is not intended to develop new and exciting methodologies for conducting sociolinguistic or sociophonetic research. Rather, it aims to make use of established methodologies across a range of variables, and to make inferences about social practice based on the findings. This is not to say that there is no innovation in this project: in chapter six I develop a measure of comparison to consider multiple variables simultaneously, and one of the larger aims of this project (as discussed in $§ 1.4 .4)$ is to explore the potential of drawing on the linguistic practices of liminal speakers to refine our understanding of the classification of sociolinguistic variables. By and large, however, this project relies on the decades of experimentation and innovation that have preceded this project, and the technological and analytical advances developed by previous researchers. This is of course true for almost all research, but perhaps particularly so for this project, where the object of study is the social backdrop against which linguistic variation is played out, rather than the specifics of the variables under investigation, or indeed the methods used in their analysis.

\subsection{Data collection}

In variationist sociolinguistics, the ideal object of study is vernacular speech, the most un-self-monitored style of speech in a person's repertoire (e.g., Labov 1984; Tagliamonte 2006). Of course, getting access to the vernacular is not always easy: the act of recording someone is likely to affect how they speak, and one of the principal datacollection methods used - the sociolinguistic interview - is inherently not a particularly natural milieu, as the presence of recording equipment can exert an influence on people's linguistic practice. The most genuinely vernacular speech is likely to be found by surreptitiously recording people going about their ordinary business, unaware that they are being studied. This, of course, would be enormously unethical, and so a standardised 
procedure has been developed in sociolinguistics to elicit styles of speech that approximate the vernacular. This section will describe the sociolinguistic interview as it is used in variationist research, address the methodological departures that I have taken from this standard model, and comment on my role as researcher in the interviews that comprise the ATC.

\subsubsection{The sociolinguistic interview}

The sociolinguistic interview, as an analytical object and methodological framework for quantitative variationist study, was formalised by Labov $(1963 ; 1966)$ in his work on Martha's Vineyard and in New York City, based on methods already in use in dialectology (Labov 1984). The aim of this interview structure was to develop corpora that were consistent and comparable, representing a range of speech styles for each individual. This interview schedule includes structured demographic questions, semistructured question-and-answer about growing up in the target neighbourhood, lessstructured stories elicited around common themes (in particular, sex, danger of death, and moral indignation), a read passage, a word list, and minimal pairs targeting particular phonetic contrasts. These various interview modules were designed with the express purpose of collecting data representing different degrees of formality and self-monitoring on the part of the speaker, and are well-suited to the kinds of linguistic investigations pursued by Labov and his colleagues.

However, this approach is not necessarily the best fit for every investigation. Differences in community, differences in research agendas, and differences in interviewers may make some changes to the general format advisable. For example, if the language variety under investigation is not characterised by specific vowel contrasts (e.g., neutralisation of vowels before a coda-1), then minimal word pairs may not provide anything useful to the investigation. There are more pragmatic points of difference, as well. With this project, where I was working with marginalised people who may have been survivors of violent attacks or sexual assaults in the past, I elected not to ask about potentially life-threatening situations. This project is likewise not directly concerned with styles of speech across the formality spectrum, so although there was a more structured question-and-answer portion to the interview and a short read passage, the content of the conversational portion of the interview was not as structured. The conversation was not steered towards hot-button topics (e.g., religion, politics) that would elicit a more rehearsed speech style; instead, the topic of conversation was allowed to drift naturally, and common areas were recurrent across interviews: travel, family, school and work, and hobbies. These common subjects make for a high degree of comparability between participants, as the style of speech stayed fairly consistent throughout: friendly and casual.

Interviews were recorded on a Sony Linear PCM (PCM-M10) digital recorder, with a lapel microphone clipped to the participant's shirt. The recorder had a remote 
control, so I was able to leave the device somewhere unobtrusive, and operate the controls without drawing attention to it. This hopefully helped the participants forget that they were being recorded, and promoted a more casual mode of speech.

\subsubsection{My role as interviewer}

Some variationist sociolinguists recruit community members to act as interviewers on their behalf, on the basis that people will be less guarded in their conversation if they are speaking to someone they know well (and, presumably, like). While this certainly has benefits from the point of view of accessing informal speech styles, there are also methodological drawbacks. Using different interviewers for different participants can introduce a level of randomness, based on the styles of the interviewers involved, and how skilled they are at maintaining an appropriate tone throughout the interview. Community members may also have wildly different relationships with participants, which can add another level of complication to already quite complicated data.

For this study, I acted as the interviewer for all of the recordings, which allowed a higher interactional consistency. For the majority of the queer and trans participants, I had been conducting loose ethnographic observations (Clifford's (1997) "deep hanging out", cited in Geertz 1998) over a period of several months, so I was at least a trusted acquaintance. For participants recruited through other people (participants or brokers), I was vouched for by someone they trusted, so while they may have been guarded at the outset, they were at least not suspicious of me or my motives. For those who were recruited more directly through an appeal for volunteers, I established a friendly interactive style through email and on the phone before we met, so again, the tone at the start of the interview was not steeped in suspicion. As the interview progressed, everybody seemed to relax, and within fifteen minutes of the start of the interview, the tone had become very conversational. Only one participant failed to relax noticeably throughout the interview, so their data was not included in the analyses that follow.

Of course, as a Canadian, I do not speak the same variety of English as my participants, which is another potential source for complication in the data. Hay, Drager \& Warren (2009) found that New Zealand speakers varied their production of the NEARSQUARE merger in response to experimenters' accents, suggesting that speakers adapt their production to what they think will be more comprehensible to their interlocutor. However, to the extent that this is systematic, the fact that my accent remained consistent across all participants means that this should not unduly affect the within-corpus comparability. More generally, this may affect how directly comparable this study's findings are to those of previous studies, but that is a separate issue.

Finally, there is the question of how participants perceived me socially, within the matrix of gendered identity that I was exploring. Queer and trans participants knew me through my involvement in those communities, so they would have seen me as part of 
their in-group. Some of the straight participants would have known that I identify as queer, based on the networks through which I met them, but others certainly seemed to see me as straight, with an assumption of shared experience and social expectation. Here, my status as a non-New Zealander may have provided a degree of camouflage: although my presentation does not particularly index queerness in a Canadian context (I do not exactly broadcast heteronormative masculinity, but nor do I come across as exactly flamboyant), in New Zealand, my accent and body language may be interpreted more as Canadian (or at least, non-NZ) than queer. While there will certainly be differences in how my various participants perceived me and responded to me, on the whole I believe that there is enough consistency across interviews to allow for the types of analysis that follow.

\subsubsection{The structure of the interview}

Interviews were generally around 1.5 hours in duration, although some were closer to one hour (generally due to time constraints on the part of the participant) and others ranged to well over two hours. The interviews were conducted wherever the participant felt most comfortable: often this was at their home, although some preferred to meet somewhere neutral (a small office on the university campus was a popular second choice, or in a public space such as a park or a cafe); interviews where the background noise presented a problem from an acoustic point of view were not included in this project; this excluded two interviews with younger trans men.

There were four broad phases in the interview. Each began with a relatively formal tone: I went over the consent form and participant information sheet with each participant, and answered any questions they had. Next was an extended period of free conversation, that tended to stick to relatively neutral topics (travel, family, hobbies, work), with no particular agenda on my part except to elicit casual conversation. Many of the participants had travelled abroad, either with their jobs, as part of school, or on a gap year, and were happy to tell stories of their adventures. People were also happy to tell me where in New Zealand I should make a point of visiting; here my foreignness was also useful, as my ignorance encouraged more expansive descriptions. The free conversation portion of the interview was the longest, and I aimed to have at least fortyfive minutes of usable conversation for each participant, excluding the initial ten-tofifteen minutes of conversation, which were excluded from analysis on the basis that they were likely to be less unguarded than later in the interview.

The third section was a semi-structured discussion of language and gender: did the participant have any sense of differences in how men and women use language, or queer and straight people, or other groups of people? As much as possible, I tried to use open-ended questions without introducing any terms that weren't first used by the participant, as I was hoping to gauge their mental maps of gendered space and wanted to impose as little external structure on that mental construct as possible. For some 
speakers, when a series of open-ended questions elicited nothing, I did ask slightly more pointed ones, such as Some people think women go up more at the ends of sentences. Have you ever noticed anything like that? What do you think about it?, which proved to be good starting-off points for the kind of discussions I was hoping to get going. Although the participants knew I was broadly interested in language and gender, the exact analytical and methodological approach I was taking were left under-specified in the information sheet and during the first and second phases, because I wanted to discourage participants from self-monitoring their speech style as much as possible. It is fairly standard practice in variationist sociolinguistics (e.g., Tagliamonte 2006: 39) to leave explicit metalinguistic or metapragmatic questions until the end of the interview. This is largely because this more reflexive perspective on the part of the participant can actually feedback into their language use in the moment (i.e., if someone is thinking about whether use of a particular feature indexes gender, then they are more likely to be aware of whether or not they themselves do it, and to manipulate their speech style in a particular direction).

The final module of the interview schedule was to record a short reading passage (which ended not being used in this project, due to time and space constraints) and to collect demographic information. These two components were not always presented in the same order: in some cases, the discussion on language and gender led naturally into the demographics section (if, for example, participants were talking about their identity, it was natural to collect that information at that point), and in others it seemed to flow more seamlessly into the read passage (if a participant started talking about differences in style or formality, it made sense to collect the read passage first). Since neither of these two components of the interview are particularly dependent on natural flow of conversation, differences in the order of presentation for these two components were deemed inconsequential to the overall comparability of the interviews.

\subsection{Identifying and working with extracts}

Once the interviews are collected, the audio material still has to be turned into useable data for linguistic analysis. Given the length of most of the interviews, and the range in those lengths between participants, I decided to consider only twenty minutes of each interview: with 46 participants, this is still a sizeable data set to work with, particularly for the phonetic variables. This section will describe the process of identifying suitable extracts, and producing the word-level transcript. The further data handling approaches needed to prepare the files for analysis will be covered in the next two sections. 


\subsubsection{Selecting extracts}

In the interests of having stylistically comparable data sets across participants, extracts were chosen that represented as close to free and casual speech as possible. This was determined by looking at the topic of conversation, general fluency (in terms of uninterrupted speech, rather than any kind of analysis of the complexity of the utterances - indeed, un-self-monitored speech is often characterised by corrections, false starts, and incomplete sentences), and non-linguistic cues such as laughter. Generally speaking, I chose sections where my contribution as interviewer was restricted to minimal responses, although in some cases it was necessary to make more substantive contributions to the conversation to keep it flowing naturally.

As much as possible, I selected twenty consecutive minutes of conversation, as this is a more accurate snapshot of a speaker in casual conversation than choosing a series of independent, temporally dislocated samples of speech. However, with some of the interviews, this was not possible - for example, due to interruptions, change in topic to something less suitable for an analysis of the vernacular, or instances of the participant asking direct questions of me. When it was necessary to use shorter stretches to make up the twenty minutes, I tried to choose selections from the interview that matched in topic and tone, and that were as long as possible. The extracts from five interviews were taken in two parts, each around ten minutes, and one interview was sampled from four places, because of a dog that interrupted and had to be dealt with by the participant, and because of a phone call. The majority of the interviews were conducted one-on-one, although three of the interviews comprised romantic couples: Ned and Patrick (older queer men), David and Noel (younger queer men), and Hugh and Linda (younger and straight). For these joint interviews, forty minutes of conversation were selected, chosen as much as possible to have roughly comparable contributions from both participants. With Ned and Patrick, the data in the forty minutes was slightly biased towards Ned (who generally was the more talkative of the two), so an additional three minutes of Patrick's speech were added so that he was not too underrepresented in the data.

As well as controlling for narrative content and casual tone, it was also important to make sure that the acoustic quality of the recording was high enough to allow phonetic analysis. For the most part, the interviews were collected in quiet places with minimal background noises, but when the location was someone's home, for example, there was always the possibility of an audible interruption. Segments of interview with many such distracting noises were excluded; where these noises were minimal and isolated (for example, a door slamming), those particular few seconds of the interview were coded for non-analysis in subsequent steps. 


\subsubsection{Transcription}

Once an appropriate twenty minute excerpt had been chosen for each interview, that excerpt was transcribed in ELAN (Sloetjes \& Wittenburg 2008; Language Archive 2015). ELAN is a suite of software designed for linguistic analysis by the Max Planck Institute for Psycholinguistics, and allows the user to segment, transcribe, and annotate both audio and audio-visual data. In this instance, the interviews were chunked into shorter segments, roughly corresponding to breath units, and transcribed as closely as possible. Each speaker (one or two participants, plus me as the interviewer) was transcribed on a separate tier, to allow for easier data manipulation further on. Instances of overlapping speech, mainly minimal responses from me or interruptions from each other when participants were interviewed together, were marked for later exclusion.

In transcribing the extracts, certain spelling conventions were followed, based on the requirements of FAVE (Rosenfelder, Fruehwald, Evanini \& Yuan 2011), the automatic alignment programme chosen for this project (see §3.5.1). As a rule, however, the extracts were transcribed with minimal punctuation, and no attempts were made to correct slips of the tongue or speech errors, to more authentically represent the spontaneous nature of the speech. Uninterpretable utterances were coded for later exclusion.

\subsection{Corpus linguistics tools: looking for adjectives}

The first variable explored in this thesis is adjectival modification, either intensification (scaling up the intensity of the adjective) or moderation (scaling the intensity down). With lexical variables, data can be extracted from the transcripts: there is no need to work directly with the audio material. However, as the transcripts yielded approximately 120000 words, a manual inspection for intensifiable adjectives would have been very time-consuming, particularly when there are corpus linguistics tools available that are designed to work with written data. It is in principle quite straightforward to use corpus linguistics tools to locate particular words, or indeed types of word, within that corpus. This is intended to be faster than identifying appropriate adjectives by hand, although in practice, part-of-speech (POS) taggers are not wholly reliable when working with spontaneous speech data, as the incomplete sentences and false starts typical of spoken language seem to interfere with the syntactic modeling.

The TreeTagger tool (Schmid 1994), included as part of UAMCorpusTool (O’Donnell 2008a, b), proved better at handling the type of data that comprises the Auckland Trans Corpus than the Stanford tagger (Toutanova, Klein, Manning \& Singer 2003), which was also included in UAMCorpusTool. TreeTagger identified more adjectives overall, but also incorrectly identified fewer non-adjectives, although both produced lists that included many instances not only of adjective-type words used as 
some other part of speech (e.g., black as a noun), but also words that are not adjectives in any context (e.g., yeah). Non-adjective-type words used as adjectives were also excluded from the TreeTagger list, so after searching for everything tagged as an adjective, a second search was conducted to identify anything tagged as a past-tense, past-participle, or present-progressive verb, as the POS tagger did not perform consistently in accurately identifying these forms when used as adjectives (e.g., overbaked, laughing). This list of verb forms was manually examined to exclude non-adjectival uses, and the resulting data file served as the starting point for the analysis of adjectival modification discussed in chapter four.

UAMCorpusTool has a limited window on the linguistic environment, constrained to plus and minus nine words of the identified target. This is because the types of questions asked by corpus linguists are often collocational, so corpus tools are designed for an examination of nearby contexts, rather than by syntactic dependency. For many of the adjectives found, the NP head being modified (whether attributively, as in the blue chair, or predicatively, as in the chair is blue) was located within nine words of the adjective, so the relevant context was generally identifiable from the UAMCorpusTool's output. In some cases, however, the necessary information was not recoverable, and in these cases, I searched for the target string of words in the transcript, and coded the tokens appropriately. See $\S 4.2 .3$ for more detail on the adjectival coding protocol.

\subsection{Phonetic analysis tools}

While the lexical variable of adjectival modification lends itself to working directly with the transcripts, the two remaining variables under investigation in this project are phonetic, and therefore need some additional preparation. The process of any kind of acoustic analysis of phonetic detail is incredibly time-consuming, but can yield more robust results than relying solely on impressionistic coding of segments. To that end, several programmes have been developed to aid in automatic analysis of phonetic segments; the first step in any of these is producing a reliable transcript that has been time-aligned down to the segmental level. Once we have the transcript aligned with the audio recording, we can take advantage of other automated and semi-automated tools to identify and extract the phonetic data that we want to analyse.

This section will first discuss the automatic alignment programme used in this study, and then will look at two extraction tools: one for the identification and measurement of sibilants (see chapter five) and one for the study of vowels (see chapter six). We will first consider the process for vowels, as this mirrors the chronological work-flow of the project, which in turn reflects the most efficient handling of the data. 


\subsubsection{Automatic alignment tools}

There are currently several automatic alignment (also known as forced alignment) suites of software available for linguistic analysis. Two such programmes were considered for use in this project: FAVE (Rosenfelder, Fruehwald, Evanini \& Yuan 2011), and MAUS (Schiel 1999). Both are programmes that have been designed expressly to handle free conversational data, and both are built on technically comparable platforms. At their core, these programmes consult a reference dictionary to produce a list of possible realisations for each word in the transcript, compare the phonetic properties of the sound file against expected values, and align segments to stretches of the recorded data. Neither programme is $100 \%$ accurate, of course, and both entail some hand-correction of the alignment. In particular, both programmes have difficulty handling overlapping speech, as the mapping of predicted waveform to actual waveform is complicated by the presence of two separate speech signals in one sound file.

The main advantage of MAUS over FAVE is that MAUS has access to a dictionary of New Zealand English, so it is less likely to misidentify or mislabel segments based on their phonetic properties. FAVE was developed at the University of Pennsylvania, and is consequently optimized for American English. However, since both programmes allow a range of possible pronunciations for each word, and selects among these on the segmental level based on the acoustics, both programmes essentially offer a phonetic alignment rather than a phonemic one, particularly with respect to vowels, which is the source of many of the differences between American and New Zealand English. Because the sociophonetic analysis in this study requires a comparison of phonemes rather than allophones, hand-correction of the vowel category is necessary in either case.

Another potential difference between General American English and New Zealand English that may have an effect on the accuracy of segmentation is the interaction of prosody and other suprasegmentals such as stress. If the rhythms of the two varieties of English are sufficiently different, FAVE might be predicted to misalign more New Zealand English vowels than MAUS would. To test whether this would have to be a consideration in choosing between the two programmes, I aligned the same sample of speech in both programmes, and compared the outputs. The output file from MAUS required approximately the same amount of hand-correction as that from FAVE, but had the disadvantage of creating a separate output file for each speaker, where FAVE produced both speakers on separate tiers of the same file. In order to identify instances of overlapping speech in MAUS-aligned files, then, two separate alignments would have to be produced, then integrated: three steps as compared to one in FAVE. On a small scale this would not be overly taxing, but with forty six interviews to align, this would add considerably to the workload of preparing the interviews for automatic measurements.

Three other advantages offered by FAVE over MAUS are error correction, stress assignment, and vowel extraction. Where FAVE encounters a problem that it is unable to 
align, it returns a file to the user with an explanation of what the problem is, and more importantly, where in the file the error occurred (i.e., a time-stamp and segment identifier in the text grid file). This makes it very easy to correct the error by hand. By comparison, when MAUS encounters a problem, the error message it provides the user is not particularly helpful in diagnosing the problem unless the user is well-versed in the programming language and back-end structure of the programme, which I am not. Error messages had to be related via email to the programming team behind MAUS, and then I had to wait for a response before continuing. FAVE proved to be considerably more user-friendly in this regard.

The dictionary used by FAVE in the alignment process includes word-level stress on a syllable-by-syllable basis, which MAUS does not. This means that the output from FAVE identifies unstressed syllables explicitly, which is very useful in separating out which vowels to measure. MAUS uses different phonetic symbols for stressed and unstressed realisations of a given vowel, which is useful but can be more confusing, as a particular symbol may be used as an unstressed realisation of a phoneme in one word, and as a stressed vowel in another. This means that I could not simply bulk-sort the vowels at a later stage, and identify which were stressed and which were not, without recourse to examining the context of each potentially unstressed vowel.

Additionally, the FAVE suite is in fact two programmes in one: a forced aligner, and an automatic vowel extraction and measurement programme. This means that the output of the alignment process is formatted correctly to interface with the measurement programme, without further processing or reformatting, besides the elimination of overlapping speech, which is required with both FAVE and MAUS in any event. The output of MAUS, by contrast, requires considerable scripting in Praat (Boersma \& Weenink 2015) in order to automatically measure the vowels. On balance, then, I decided that FAVE was the better programme for my specific purposes.

Each transcribed extract was aligned using FAVE, and the output file was then hand-corrected. At this stage, hand-correction involved scanning the alignment and shifting the boundaries that were both obviously erroneous and easily corrected. At some points in the transcript, however - where, for example, a speaker spoke particularly quickly or not very clearly - the misalignment extended over several segments (often over several words), and it was simpler at that point to code those segments for exclusion from further analysis. Similarly, instances of overlapping speech were also recoded by hand to be excluded, as were instances of interference from some other source of noise (e.g., dogs barking, phones ringing, doors slamming).

\subsubsection{FAVE-extract}

As mentioned above, one of the main advantages of FAVE-align is that it is designed specifically to work in conjunction with FAVE-extract, an automatic vowel-measurement programme. The output from FAVE-align is correctly formatted for FAVE-extract, and 
can be fed directly into the extraction and measurement suite. Sections that had been identified and coded for non-extraction (see above) were ignored by the extraction algorithm.

The output from FAVE-extract provides a lot of data about the vowels: where each vowel occurred (a time-stamp in the recording, the carrier word, the lexical environment, and the phonetic environment), whether it was stressed or not (at the lexical level - phrase-level stress cannot be extrapolated based on dictionary entries), and formant measurements across several points in the vowel. A detailed discussion of the vowel measurement points adopted in this study is given in section $§ 6.2$.

\subsubsection{Shortcomings of FAVE}

As with any automated process, there is opportunity for error to be introduced into the data. Segmental alignment is never one hundred percent accurate, particularly when one considers the effects of coarticulation with preceding and following environments, which can lead to disagreement among human aligners as well. However, since FAVE was designed specifically to investigate vowels, its settings are such that they seem to be quite vowel-conservative. That is to say, if there is an acoustically complicated vowel onset, for example, FAVE errs on the side of vowel - which means in practice that the boundaries for some vowel onsets are a few milliseconds later than they might be if they were hand-identified, and likewise, some vowel codas are marked a few milliseconds earlier than they might otherwise have been. This means that the vowel duration as measured by FAVE is likely to be some milliseconds shorter than the actual acoustic duration of the segment. With this in mind, I chose not to consider vowel duration in this analysis, although vowel length is certainly a distinctive feature in New Zealand English (e.g., Warren 2006a) and may also be used for sociolinguistic acts of identity.

The tradeoff for this shortened vowel, however, is a greater likelihood that the segment identified as a vowel for the purposes of measurement is actually a stable set of formants that are deterministic of the vowel itself, and not being interfered with by preceding or following segments. Of course, the phonetic environment is still going to have an effect on the phonetic realisation of a vowel, but this type of systematic effect on the first three vowel formants is a distinct phenomenon from the type of acoustic noise that can occur at segmental transitions and which can render it very difficult to identify the formants at all.

Misidentification of formants is another notable concern, as the algorithms used to identify and therefore measure the formants cannot possibly account for all the potential variation inherent in a recording of natural speech, particularly when that recording was made outside of an acoustically controlled space, such as a phonetics laboratory. Spectrographic analysis relies on the identification of bands of concentrations of energy, and where these bands are too close together, for example, or not particularly concentrated, the computer program responsible for identifying them can have 
difficulties. Changing the number of formants that the computer program anticipates finding can affect the accuracy of the measurements; the findings from Evanini's (2009) doctoral research have been incorporated into the FAVE software, in an effort to maximize the number of measurements possible while minimizing the rate of formant misidentification. While there are doubtless improvements being continuously made to these processes, this study used the most up-to-date tools available at the time the study was conducted.

\subsubsection{Semi-automatic tools for sibilant analysis}

While the popularity of vowels as an object of linguistic study has resulted in the development of several automated toolkits designed for this specific purpose, the study of sibilants has not seen a comparable spate of software solutions. However, there are semiautomatic processes that have been developed for general phonetic study, and which are applicable to the examination of fricatives in this project.

\subsubsection{Praat}

There are a number of software options available for phonetic analysis. In sociophonetics, two common ones are EMU (Winkelman, Jaensh, Cassidy \& Harrington 2016) and Praat (Boersma \& Weenink 2015). EMU is run through the statistical platform $\mathrm{R}$ ( $\mathrm{R}$ Core Team 2013), while Praat is a standalone application. Although there is considerable overlap in their aims and outputs, they differ substantially with respect to user interface and scripting language (Williams 2008: 173). EMU is particularly wellsuited to detailed structural phonological analysis, which is not a requirement for this project; my interest is more on a phonetic level. As I am already a confident user of Praat, and as it meets the technical requirements for this project, I elected not to switch to EMU.

With the files already time-aligned to the segmental level (see $\$ 3.5 .1$ above), it is simple to use a script in Praat - a small set of instructions to carry out a series of algorithmic operations - to automatically identify and measure fricatives. A high- and low-pass filter was applied to the sound file, to restrict the domain of analysis to the set of frequencies relevant to speech, and to exclude the range of frequencies over which F0 would be a factor in the case of the voiced $/ \mathrm{z} /$. This minimises the effect of any extremely high or extremely low frequencies on Praat's automatic measurement system.

Because the FAVE-align system was designed for maximum accuracy in identifying and segmenting vowels, it is possible that the start and end points of the fricatives is not entirely faithful to the spectrogram. As the spectral measurements are taken at the midpoint, and averaged over the central $40 \mathrm{~ms}$, any slight offset of the start and end points of the fricatives will not have an enormous impact on the measurements of spectral moments. However, this will likely have an effect on duration measurements, where a difference of a few milliseconds on either end may represent a significant 
proportion of the entire segment. Due to the large number of tokens extracted and measured using automated techniques, it is not feasible to manually check alignments for all of the fricatives, or even all of the sibilants. So although segment duration may very well have an effect on its spectral properties, it is not possible to reliably obtain durational data for the sibilants in this data set. The role of duration on spectral moments will be investigated later, using a smaller subset of this data.

\subsection{Analytical approaches}

For all of the quantitative analyses that follow (in chapters four, five, and six), broadly similar analytical approaches were adopted, although the particular factors under consideration differ between variable systems (i.e., adjectival modification, sibilants, and vowels). The discussions draw on both descriptive statistics and multivariate analyses, as each perspective can contribute to our understanding of the observed variation.

\subsubsection{Descriptive statistics}

The first and easiest way to look at data is to consider the straightforward distributional results: how does the target variable pattern across the categories of interest? This gives us our first impression, and can often be represented visually, which can be easier to interpret at a general level.

We can also do simple statistical tests with distributional data, to interrogate the degree of difference between our categories. Continuous variables can have their means compared, to determine if the differences we observed are likely representative of real differences in the data, or are more likely to be the result of how we sampled our data. The particular statistical approaches adopted will depend on the variables, as different types of data may be better-suited to some tests than others. One test that we will use with some regularity in this thesis is the Analysis of Variance (ANOVA), which is designed specifically for multiple comparisons.

\subsubsection{ANOVA}

If we were interested in comparing the means of only two groups, then a simple Student's t-test would be ideal; because we have eleven groups that we want to compare, however, the t-test stops being a useful tool. With each additional group that is added to the pool of comparisons, two things happen:

(1) the number of t-tests that needs to be performed increases geometrically

(2) the probability of getting a Type I error (incorrectly rejecting the null hypothesis and claiming a significant difference when there is none - in effect, a false positive) increases rapidly

The first of these is arithmetically unavoidable, but computers have made the task of 
doing many calculations very easy.

The more fundamental problem with this approach is the second point made above: the increase in Type I errors. For a given alpha (the value usually adopted in social sciences is $\alpha=0.05$, which means that the probability of getting a "false positive" is $5 \%$, or one in twenty), each comparison will increase the likelihood of getting a false positive. The formula for calculating the familywise error rate $\left(\alpha^{\prime}\right)$ is:

(1) $\alpha^{\prime}=1-\left(1-\alpha_{\text {per.comparison }}\right)^{k}$

(Cramer \& Howitt 2004: 64) where $\alpha_{\text {per.comparison }}$ is 0.05 , and $\mathrm{k}$ is the number of pairwise comparisons as calculated above. With eleven groups, this works out to $\alpha^{\prime}=0.94$. In other words, it is almost certain that we will have a number of false positives, but we have no way of knowing which ones these might be.

To get around this problem of multiple comparisons, statisticians developed the Analysis of Variance (ANOVA) test, which is formulated specifically to compare multiple means. It calculates the probability that at least one of the pairwise comparisons is significantly different at the $\alpha=0.05$ level. Although this in itself cannot identify the comparison(s) where the difference is significant, several post-hoc tests have been designed to do exactly this. In particular, Tukey's HSD (Honest Statistical Difference) is a useful method for comparing the means of multiple pairwise comparisons, and this test can easily be included with an ANOVA, using the statistical analysis programme SPSS (IBM Corp 2013).

\subsubsection{Tukey's HSD and homogeneous subsets}

The overall ANOVA indicates whether there are any significant differences between the groups being compared, but does not actually indicate for which groups the differences are significant. For this extra bit of information, we will use the Tukey's HSD, which provides two useful pieces of distributional evidence: it indicates for each pairwise comparison within the group whether the difference is significant, and it provides a table of homogeneous subsets (clusters of groups within which the differences are nonsignificant).

The pairwise comparisons will be particularly useful for our analysis of vowels, when we develop a metric of overall similarity (see section §6.3.6). The homogeneous subsets will be used with both sibilants and vowels, as this perspective on the data provides a useful indication of how (un)evenly the groups are distributed within the bounds of the particular variable.

\subsubsection{Multivariate analysis}

While an ANOVA followed by appropriate post-hoc tests can tell us a good deal about the distribution of the data, there are limits to how generalisable these observations are because means testing assumes that all other factors have been held constant. That is to say, there is an assumption that the only source of meaningful difference between one 
set of measurements and the others is the thing that you are testing. This makes meanstesting a very powerful tool in controlled laboratory settings, where the data can be collected in such a way that all other potential sources of variation can be controlled for, or at least managed systematically. With naturalistic data, however, the picture is much more complex, because we are not able to constrain all of the possible confounds, and the data is almost guaranteed to be unbalanced, non-independent, and relies on multiple measurements from different individuals (Johnson 2009) - making it inherently difficult to model statistically. We have no control over what words our participants will produce, or what the discourse context for those words will be, or the speaking style being used at any given moment. We can approximate some degree of comparability by choosing which parts of the interview we will examine, but we still need a different analytical approach to take into account the effect of multiple sources of variation. Fortunately, sociolinguists have developed a multivariate approach that is particularly well-suited to the types of spontaneous speech that tend to comprise our data sets.

Multivariate analysis in sociolinguistics has gone through several iterations of variable rule analysis and regression modeling. One of the early variationist statistical programmes was VARBRUL (Sankoff 1975, 1988), followed by numerous versions of Goldvarb (e.g., Sankoff, Tagliamonte \& Smith 2005). R-Varb (Paolillo, n.d.) moved the functionality of VARBRUL into the R platform (R Core Team 2013), and the current suite of software is Rbrul (Johnson 2009). The goal of this kind of analysis is to evaluate and identify "which factors significantly affect the response variable of interest, in what direction, and to what degree" (Johnson 2009: 359). In other words, it attempts to disentangle the effects of several factors that act simultaneously on the surface realisation of a variable - precisely what the means-testing was not able to do.

Throughout this thesis, we will focus our discussion on the role of the social factors within the larger system of structural variation, but we do so having made an attempt to filter out the effects of other sources of variation. Of course, we are limited to identifying the effects of the factors that we have chosen to code for and to include in the statistical model, which in turn is limited by the automated and semi-automated tools that we have used to extract the data from the recordings and the transcripts. There are always limitations to any analysis, and we need to bear those limitations in mind as we consider the implications of our analysis.

Throughout the variable discussions in the following chapters, speaker has always been included as a random effect in the models, although word has not been (for both principled and practical reasons which are beyond the scope of this discussion). For each analysis, statistical runs were conducted using gendered identity as a composite category, as well as those where it was deconstructed into its three components: age [older/younger], identity [straight/queer/trans] and gender [man, woman], and modeled with possible interactions between these groups. The best model between competing candidates was selected using the log-likelihood test incorporated in Rbrul. 


\subsection{Variable-specific particulars}

In the preceding sections, I have laid out the broad methodologies adopted for this project: the collection and transcription of the sociolinguistic interviews, the selection of comparable extracts, and the tools used to facilitate the extraction and analysis of data.

However, because this project looks at three systems of variation (one lexical and two phonetic), there are particular methodological approaches that are unique to each, and it makes more sense to discuss those details as they become relevant. So while this chapter has provided an introduction to the approaches taken in this thesis at a very broad level, it is not intended to describe the specifics for each variable. This more detailed perspective will be provided within the framework of each of the following three chapters: adjectival modifiers, sibilants, and vowels. 


\section{Chapter four: Adjectival modifiers}

\subsection{Introduction}

The system of intensifiers in English has been the topic of linguistic commentary and analysis for well over a century, passing through phases of observation and commentary (e.g., Stoffel 1901) and semantic description and analysis (e.g., Bolinger 1972) before coming to the attention of variationist sociolinguists (e.g., Ito \& Tagliamonte 2003; Tagliamonte 2008). One of the reasons that it is of perennial interest is the fact that it shows remarkably rapid and dynamic change (e.g., Quirk, Greenbaum, Leach \& Svartvik 1985; Peters 1994), and as such provides an opportunity to not only observe processes of language change in action, but also a chance to see how social groups can make differential use of the options available to them.

This chapter explores the use of intensifiers and moderators in the Auckland Trans Corpus (ATC), using a combination of distributional data and, where numerically possible, multivariate analysis to tease out the social factors that motivate variation.

\subsubsection{What are intensifiers?}

Intensifiers are an easy target for variationist sociolinguists, because they occur so frequently in spontaneous speech. The term intensifier has been used in slightly different ways by different researchers, depending on the question they were investigating. Generally, they are taken to be pre-adjectival modifiers; Bolinger (1972: 17) classes four types, based on the direction and magnitude of the scaling effect they have on the adjective:

1. boosters ("upper part of a scale, looking up")

2. compromisers ("middle of the scale, often trying to look both ways at once")

3. diminishers ("lower part of the scale, looking down")

4. minimisers ("lower end of the scale")

Ito \& Tagliamonte (2003: 258) add another to this list, maximisers, which would correspond to "upper end of the scale" in Bolinger's taxonomy.

Generally, sociolinguists (e.g., Ito \& Tagliamonte 2003; Tagliamonte 2008; Scandrett 2012) have been concerned with boosters and maximisers, which they tend to refer to simply as intensifiers; the downward-scaling modifiers - downtoners (Quirk, Greenbaum, Leech \& Svartvik 1973) or moderators - have been largely ignored, although see Holmes (1995) and Holmes \& Stubbe (2003) for a discussion of hedges and moderators as politeness strategies.

For the purposes of this study, we will define intensifiers as adjectival modifiers 
that scale up the intensity of the adjective, as in (1) below, and moderators as those that scale down the intensity of the adjective, as in (2). This two-way distinction, which collapses several intermediary classifications, follows Biber, Johansson, Leech, Conrad \& Finegan's (1999) system, and is a more appropriate taxonomy given the size of the data set available for this project (see $§ 4.2 .1$ below).
a. you've still got the really weird people (Scott, ysm)
b. it's a very nice place (Stuart, yqm)
c. the establishment of boundaries is so important (Diane, osw)
d. I consider myself extremely fortunate (Graeme, osm)
e. it's totally fine (Adam, ytm)
f. it was just so miserable all the time (Linda, ysw)
g. it was just a really really terrible dark time (Roslyn, ysw)
a. there are bits of it that are a bit brash and ugly (Ned, oqm)
b. I could have had a good enough rank score (Lawrence, ysm)
c. something that makes them kinda likeable (Alison, osw)
d. this just sort of fairly basic stuff (Lisa, otw)
e. $\quad$ it was sort of slightly questionable (Ned, oqm)

Note that in (1f), just is considered part of the adjectival modification, while in (1g) it was not; this is primarily structural, as the presence of the determiner $a$ in (1g) places just outside of the Adjective Phrase (AP), and therefore not a direct adverbial modifier of the adjective. Whether it is semantically accomplishing the same end is a different question; in the interests of comparability with other studies, however, we will restrict our analysis to AP-internal modification, and leave a more structural analysis of the syntax and semantic of AP-external modification for later research.

It also worth briefly discussing the structural difference of $(2 b)$, which is not preadjectival, as the rest of the tokens are. Adverbs in this position known as a postadjectival (Dixon 1982: 24) or postmodifying (Bolinger 1972: 97), and there is some debate as to whether these are actually within the AP or not. However, it is not grammatical in New Zealand English (or, to the best of my knowledge, in other varieties of English) to use enough as a pre-modifier, as in it's enough good as opposed to it's good enough), so the modifier obligatorily follows the adjective. This roughly parallels the restrictions on AP placement for certain pronouns in English, such as everything, anything, something and nothing, discussed in section $\$ 4.2 .3 .3$ below. Since the position of enough is invariant with respect to the adjective it modifies, we will consider its positional restriction a lexical property, and will assume that it otherwise conforms to the syntactic demands of intensifiers and downtoners (which it seems to do).

We are also including the use of litotes, as in (3), in our analysis, classed as a 
form of moderator. Litotes is a rhetorical strategy of using negation of one thing to indicate the opposite (3a), and often co-occurs with intensifiers to further the effect (3bd).

(3) a. being against a rock face when a tsunami comes is not good (Scott, ysm)

b. our lives aren't so bad, it could be much worse (Amanda, yqw)

c. it was not actually that conducive to playing rugby (Richard, ytm)

d. they were not very happy with it being a public system (Ned, oqm)

All of the examples given in (1-3) are drawn from the ATC, which shows both the productivity of the system of intensifiers and moderators in New Zealand English (NZE), as well as the range of variation possible.

\subsubsection{Intensifiers in NZE}

Perhaps the most iconic intensifier in New Zealand English (NZE) is the $\mathrm{ADJ}+$ as variant, particularly in the phrase sweet as but also said to be productive with a limited set of other adjectives (e.g., Bauer \& Bauer 2002; 2002a). It has been only incidentally attested in spontaneous spoken data, however (Petrucci \& Head 2006: 333), despite being slightly more common in written and online corpora (e.g., Sowa 2009) and quoted material in newspapers (Petrucci \& Head 2006). Unfortunately, the ADJ+as construction was only used by one speaker in the ATC (Erich, a 32-year-old trans man), so it is not possible to investigate its productivity in natural speech data with this project.

The intensifiers most commonly reported in NZE are very and really. Hay, Maclagan \& Gordon (2008: 62) note that very is in decline, and really is increasing, which was also noted in Scandrett's (2012) diachronic study of NZE intensifiers, working with recorded data from participants born between 1851 and 1984. Scandrett also notes that so has slowly been increasing over time (2012: 40-41), particularly in female speakers, and predicts that this pattern will continue (2012: 42).

Saarenpää's (2016) corpus comparison of intensifiers in NZE and Canadian English reports that very, really, so and pretty are the most common variants in NZE, with very preferred by men, and really and so preferred by women (p.52), and shows that the use of very is declining over time while really is increasing. To the best of my knowledge, there has been no variationist study of intensifiers in NZE, so distributional data will have to serve as our comparative benchmark for this study.

Both Scandrett and Hay, Maclagan \& Gordon are reporting on data from the ONZE project, which is weighted towards participants from the South Island, both rural and urban; it also spans a considerable time-depth, including speakers born in the late 1890s (Gordon, Campbell, Hay, Maclagan, Sudbury \& Trudgill 2004). Saarenpää made use of the International Corpus of English - New Zealand, which includes both spoken and written data; the spoken data, which we would expect to more closely align 
stylistically with the ATC data, is weighted towards participants from Wellington, and was collected between 1988 and 1994 (SLALS, n.d.). Given that this project deals with the variety of English spoken in Auckland in 2013-2015, we may predict certain regional and apparent-time differences in intensifier use between this study and these previous ones.

\subsubsection{Language change}

As noted above, one the reasons that variationists like intensifiers is that the processes of grammaticalisation and change are rapid enough that they can be observed in real-time with a relatively shallow time-depth. The general process of change seems to be that, as any given variant gets used more and more frequently, its sense of intensity wanes, and so new forms must be constantly introduced to pick up the communicative slack (Robertson 1954: 251 cited in Ito \& Tagliamonte 2003; Bolinger 1972:18). In fact, forms can also be recycled (Dubois \& Horvath 1999; see also Ito \& Tagliamonte 2003), meaning that older variants can be re-introduced with a new sense of intensity, not because they are new to the system as a longitudinal whole, but rather they are new to the group of speakers using them.

On top of this individual variation, there are deeper trajectories of grammaticalisation and semantic shift at work, as the original meanings of the intensifiers (e.g., very > verily 'truthfully') are lost as the set of collocational contexts expands. Tagliamonte (2008: 371) notes that, as an adjectival intensifier, very predates really by around 400 years. The variationist sociolinguistic perspective has contributed to a more detailed understanding of this process, mapping out the paths of contemporary English intensifiers - in particular, very, really and so - as their range drifts from their original semantic meanings. These systematic shifts in meaning, and the accompanying broader set of contexts observed, are discussed in detail in Ito \& Tagliamonte (2003), Tagliamonte \& Roberts (2005) and Tagliamonte (2008).

\subsubsection{Social difference}

Of more interest for this thesis is the question of social difference. Certainly, gender effects have been reported in Ito \& Tagliamonte's (2003) study of York English, and in Tagliamonte's (2008) parallel examination of Canadian English, although in both of these cases, social effects were interpreted as indicative of the progress of change underway, it being a well-established principle of variationist sociolinguistics (e.g., Labov 1990; 2001) that young women tend to be linguistic innovators under certain conditions, and to be conservative under others. However, studies looking at English intensifiers under a more nuanced gender lens have found more interesting patterns.

In Canadian English overall, young straight female speakers show a preference for really and so as intensifiers, while young straight male speakers prefer pretty (Tagliamonte 2008). In a smaller-scale study that considered the role of social network 
and community, Tagliamonte \& Uscher (2009) found that the gender differences for really were minimised within a sub-corpus of LGBT (queer) speakers, as compared to their straight peers. This points to intensifiers as a site for positioning gendered identity in spoken discourse, as lexical items are accessible for manipulation (potentially more so than segmental variables) and interpretable at a fine-grained enough level to mark social categories such as queer and straight.

Intensifiers have also been examined in the speech of transsexuals. Brown (2009) sourced data from a number of vlogs (video blogs) of Canadian trans women, which she considers comparable to spoken data in that they are unscripted, often streamof-consciousness in structure, although she does acknowledge that they are more consciously performative (i.e., recorded with an audience in mind) than conversational data. Brown reported that overall, trans women intensify less than either cissexual men or women, using Tagliamonte's corpus of Toronto English as a point of comparison. The trans women in her study exhibit lower rates of use across all of the most productive intensifiers (really, very, pretty, so), but this is particularly true for the feminine-gendered so. Hazenberg's (2012) study of queer and transsexual language use in Ottawa, Canada, found that trans men intensified overall more than any other group of speakers, and that trans women had comparable rates to cis straight women. More interesting is that trans speakers generally avoided the two most gendered intensifiers - pretty (masculine) and so (feminine), similar to Brown's findings. Brown concluded that the "majority of trans women are participating in the gendered intensifier system as they were socialized [to], (i.e., as boys/men)" (2009: 58), although Hazenberg's inclusion of data from trans men supports a slightly different interpretation. He argues that trans speakers steer clear of the strongly gender-indexing intensifiers more generally, preferring to use more genderneutral variants such as really. Both the Toronto and the Ottawa studies examining trans speakers against the backdrop of a more heteronormative speech community argue that straight men are distancing themselves from women and from queer men through their choice of intensifier and rates of use, rather than women and queer men distancing themselves from straight men.

Of course, these previous studies have examined intensifiers in varieties of Canadian English that are quite closely-related linguistically (Toronto and Ottawa being geographically near to each other by Canadian standards, and socially very similar as well). A study of intensifiers in Newfoundland English (Bulgin, Elford, Harding, Henley, Powers \& Walters 2008) - another Canadian variety, albeit one with considerably more structural difference than Ottawa and Toronto (e.g., Labov, Ash \& Boberg 2006) - concludes that the social meaning of variants is "locally constituted" (p.114), so we cannot assume that the social indexicality of pretty and so are going to be the same in Auckland as they are in Toronto. What these previous studies do tell us, however, is that intensifiers as a system are capable of encoding gender and other social information, which makes then a viable candidate for examination in this study. 


\subsubsection{A note about downtoners and moderators}

Although downtoners are a subset of adjectival modifiers parallel to intensifiers, the fact is that they seem to show very little by way of social stratification (Sali Tagliamonte, p.c. $)^{1}$, and considerably less creativity and variation in conversational use than intensifiers (Biber \& Conrad 2001). Hazenberg (2012) reported generally lower rates of use for downtoners than for intensifiers, and found that litotes was the generally preferred strategy for moderating an adjective in Ottawa English, except for trans men, who showed a marked preference for kind of and its reduced form kinda. The low number of tokens overall made a more detailed analysis impossible, but the fact that any social difference was noted raises the possibility that downtoners may also be a potential site for gendered performance in Auckland, as well.

\subsection{Methods}

The broad methodologies used in a variationist analysis of intensifiers are welldocumented (e.g., Ito \& Tagliamonte 2003; Tagliamonte \& Roberts 2005; Tagliamonte 2008), so this section will not present the approach from an entirely first-principles perspective, but will instead focus on the particulars of how these methodologies were applied in this case. Interested readers are directed to any of the Tagliamonte readings for a more detailed discussion of these methodologies and the rationale underlying them.

The main issue underpinning a variationist analysis is to clearly define the variable context (e.g., Tagliamonte 2006). Because the statistical analysis at heart is trying to model the choice of a particular variant over the non-choice of that same variant, it is not enough to account for where that variant occurs, but also where it could occur but does not. In the case of the system of adjectival intensification, it is widely accepted that the most appropriate unit of analysis is the adjectival head, which may or may not be modified. For example, something could be good, or it could be really good, or not particularly good. The simplest way to ensure that the analysis takes each of these possibilities into account is to record every instance of good, and to code it according to (among other things) whether or not it has been modified, and if it has, how. Of course, we are not particularly interested in the specific adjective good, but we can widen our net to include all adjectives, and code them using a similar principle.

\subsubsection{Finding adjectives in the interviews}

As discussed in section $\S 3.4$, adjectives were identified in the transcribed corpus

\footnotetext{
${ }^{1}$ But see e.g., Holmes (1995) and Holmes \& Stubbe (2003) for a discussion of the use of many of these variants as propositional hedges (as opposed to adjectival modifiers), where they do show some social stratification.
} 


\begin{tabular}{|c|c|c|c|c|}
\hline & intensified & moderated & unmodified & total \\
\hline oqm & 103 & 43 & 331 & 477 \\
\hline oqw & 79 & 14 & 237 & 330 \\
\hline osm & 92 & 34 & 423 & 549 \\
\hline osw & 116 & 32 & 372 & 520 \\
\hline otw & 54 & 15 & 248 & 317 \\
\hline yqm & 90 & 29 & 474 & 593 \\
\hline yqw & 80 & 37 & 326 & 443 \\
\hline ysm & 107 & 49 & 310 & 466 \\
\hline ysw & 165 & 40 & 484 & 689 \\
\hline ytm & 183 & 50 & 433 & 666 \\
\hline ytw & 26 & 3 & 201 & 230 \\
\hline total & 1095 & 346 & 3839 & 5280 \\
\hline
\end{tabular}

using the UAMCorpusTool (O'Donnell 2008a, b). In spite of complications arising from using spontaneous - and therefore frequently 'ungrammatical' - speech, a large number of adjective heads were identified, and this data set was the starting point for the analysis that follows. Of course, not all adjectives are modifiable by intensification or moderation, so the initial list of tokens was further reduced (discussed in section $\$ 4.2 .2$, below), yielding a total of 5280 tokens of adjectival heads (broken down by gender and modification in Table 4.1) after exclusions as discussed in the following sections.

It should be noted that, although care was taken to identify adjectival uses of verb forms that were not included in the initial search for adjectives, it is possible that other non-canonical adjectives were also omitted, particularly those whose structural positions could be interpreted ambiguously from a probabilistic point of view. However, rather that replicating the work that the POS software did and manually searching through the corpus transcripts for adjectives, it was decided to use the potentially reduced data set as it was. Since the same procedures were used across all speakers, any lost or overlooked data should have been evenly distributed across the corpus, hopefully mitigating any overall effect on the subsequent analysis.

\subsubsection{Identifying appropriate adjective heads}

Simply having a list of all adjectives in the corpus - and the immediate context in which they are used - is not enough for an analysis of intensifiers and moderators, since not all adjectives can be modified by things like really or not particularly. An electric stove, for example (as opposed to a gas stove) can't really be thought of as being really electric: it either is electric, or it isn't. One electric stove isn't electric to a lesser degree than another. So we are actually looking for gradable adjectives, or adjectives that are used as though they are gradable.

The classic examples of non-gradable adjectives are dead and pregnant, but in 
actual practice, they can be variably used in a gradable sense. My ancestors are dead in a non-gradable sense - my great-great-great grandmother is neither more nor less dead than my great-great-great-great grandmother. On the other hand, a car engine that sputters but won't turn over is less dead than one that won't even click when the ignition is turned. The first car is dead, but the second car is totally dead, or completely dead, or deader than dead. Likewise, a woman who is two weeks past due could be said to be extremely pregnant, particularly compared to someone who is in their first trimester. Semanticists may argue that the deadness of my great-great-great-great grandparents and the deadness of my car are two different things (e.g., Kato 1986; Kennedy 1999): the first is a literal meaning where dead $=$ not alive, and the second is a metaphorical one where dead $=$ not currently functioning; or indeed, that there is a difference between having/not having an embryo developing in your body versus looking like you do.

In a similar vein, it is possible for inherently gradable adjectives to be used nongradably. This is particularly true in fixed expressions and proper or compound nouns. January first marks the new year: it will never mark the very new year, even though my shoes can be so new that they still squeak. A television show called Incredibly Bad Girls would be a separate production from the popular British drama Bad Girls, although drinking strong coffee right before bed is probably an incredibly bad idea.

In the context of a research project such as this one, there is no tidy algorithmic procedure for isolating the literal from the metaphorical uses of any given adjective: each instance must be examined, to determine whether or not it is intensifiable as it is used in that moment. Thus an electric stove would not be included as an intensifiable adjectival head, but an electric performance would be (because it could have been an outstandingly electric performance).

Other adjectives that were excluded from the analysis included those that were incomplete or cut off (e.g., um very very litt-well very little for example) and attributive adjectives whose noun was missing (e.g., there is like this deep-seated-I don't know what happened to him). In the first example, only the second instance of little would be taken as a viable token, while the first one - which was cut off mid-utterance - would be regarded as a false start. With spontaneous speech data, such false starts and corrections are not uncommon, and in the interest of consistency of coding, it is simpler to exclude these tokens.

\subsubsection{Coding adjectives for analysis}

After the non-intensifiable adjectives were excluded from the list, the remaining tokens were coded for a number of linguistic factors that were used in the subsequent analyses. These included the type of modification; sentence polarity; the syntactic position of the adjective; the type of adjective; and the evaluative position of the adjective. These various factors are discussed briefly below. Each token was also coded for the relevant social factors particular to the speaker: their age (older/younger), their 
identity (straight, queer, trans), their gender (men/women), and the interactions of these three axes.

\subsubsection{Type of modification}

The type of modification included whether or not the adjective was modified at all, and if it was, whether it was intensified (scaled up in intensity) or moderated (scaled down). Each modified token was further coded for the specific variant used; these were later sorted into a small number of groups, based on frequency. The most common intensifiers (accounting for at least 5\% of the total intensified tokens) were really, very, so, quite, just, and pretty. The most common moderators were sort of/kind of, quite, a (little) bit, litotes (i.e., negation-as-moderator), and enough. These are discussed in more detail in $\S 4.4$.

One complicating element in this categorisation is that certain variants - in particular, pretty and quite in New Zealand English - can be used either to intensify or to moderate an adjective. In a practical sense, these are very difficult to disambiguate without recourse to the audio recordings; so for each of the pretty and quite tokens, I listened to several seconds of the interview, and made a determination based on the context in which the adjective was used and the prosodic cues of the speaker. In cases where I was unable to make a confident assessment, I played a 10 to 15 second excerpt from the interview to three native speakers of New Zealand English (ranging in age from late $20 \mathrm{~s}$ to mid 40s), and coded the token according to the majority opinion. There were fewer than ten such tokens altogether, and the three judges failed to come to a consensus in only one instance; this token was removed from analysis.

\subsubsection{Sentence polarity}

As the majority of previous studies have looked only at intensified contexts (excluding moderators or downtoners), adjectives that were under the scope of a negator have generally been excluded from analysis. This includes both intensified and non-intensified contexts: it's not good and it's not very good would be not included in such a study. This is generally done to control for the fact that litotes - the rhetorical device of using negation to lessen the impact of an adjective for more detail - can co-occur with intensification, but the effect is not to simply negate the intensified adjective, but rather to denote a different position on the scale of the gradable adjective. That is to say, not very good does not communicate a failure to be very-good; it more closely communicates a not-very degree of goodness - which is not an intensifier in the sense of something that scales up the value of an adjective, and is therefore not part of the variable context. And in order to not over- or under-represent rates of intensification in the data, if the intensified negative-polarity items are excluded, then so must the non-intensified ones.

However, since this study includes moderators - a category in which I am including litotes - we do not need to exclude adjective phrases with negative polarity. 


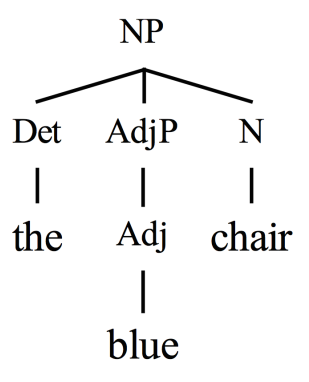

Figure 4.1: Adjective in attributive position

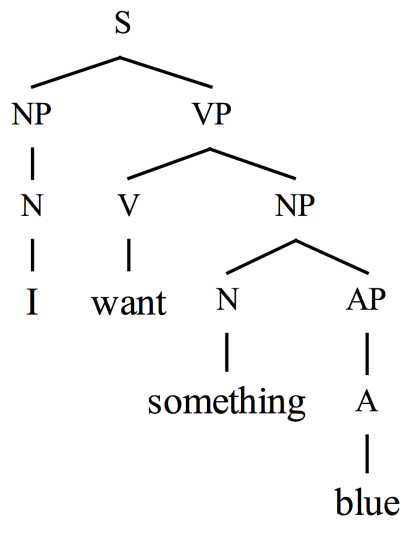

Figure 4.3: NP-internal postpositive AP

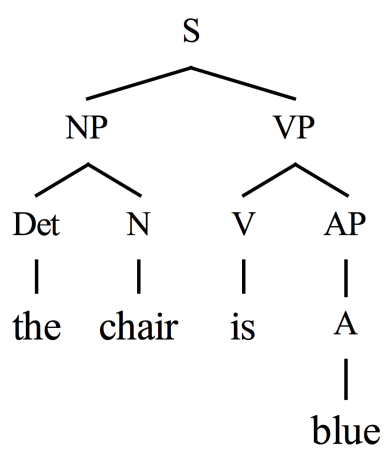

Figure 4.2: Adjective in predicative position

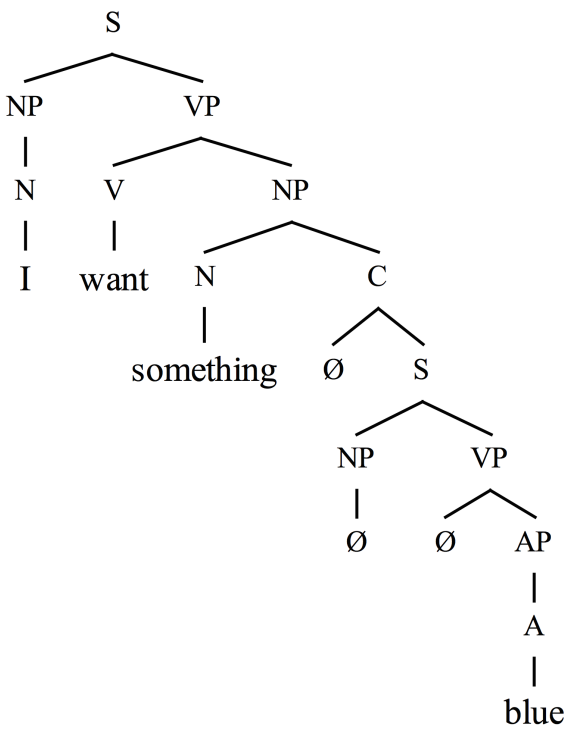

Figure 4.4: Postpositive AP as reduced relative clause 
For the negative contexts, each token was examined to determine if it was a case of litotes or a literal negation of the adjective, and coded accordingly. So if, in context, not bad was used to affirm that bad was not an appropriate label, it was coded as 'unmodified'. If, on the other hand, not bad was contextually meant to imply (kind of) good, then it was coded as litotes. In determining the communicative intent behind these cases, I went back to the audio recording, and used both prosodic and contextual cues to disambiguate the role of the negative particle, and used the same three judges as mentioned above in the pretty and quite disambiguation.

\subsubsection{Syntactic position}

Each adjective was coded for where it was positioned syntactically relative to the thing it was modifying. The two main positions in English are attributive, when the modifier occurs within the noun phrase (Brown 2006: 11); and predicative, when the modifier functions as a subject or object complement to the noun phrase (Quirk, Greenbaum, Leach \& Svartvik 1985: 417). Thus the blue chair would be attributive (Figure 4.1), while the chair is blue would be predicative (Figure 4.2).

However, there is a third possibility: postpositive, where the modifier comes after the noun it modifies, which is usually a compound indefinite pronoun (e.g., something, anything, etc.) but can also occur with a regular noun in a limited set of fixed expressions (e.g., the Governor General) (Greenbaum \& Quirk 1990: 136). An example of this is in I want something blue, where blue is modifying something. Pullum \& Huddleston (2002: 528) argue that postpositives can be thought of as being NP-internal (Figure 4.3), although others (e.g., Cinque 2010: 60; Quirk, Greenbaum, Leach \& Svartvik 1985: 418) regard postpositives as reduced relative clauses (Figure 4.4).

Since postpositives did not occur frequently enough in the data to warrant their own category in the analysis, I examined their distribution and decided that, on balance, they pattered more closely with attributive adjectives than with predicative ones, and so were recoded accordingly. This was further tested by doing two simple multivariate analyses, where all factors were identical except for the classification of postpositives, which was attributive in one case and predicative in the other; the attributive model provided a better fit for the data, suggesting that deep structure notwithstanding, people treat postpositives as a special case of attributive modification.

\subsubsection{Type of adjective}

Tagliamonte's various studies of intensifiers make use of Dixon's (1982: 15-16) taxonomy of adjectives, drawing on the fact that more delexified (and therefore further grammaticalised) intensifiers have wider collocation patterns (Partington 1993: 183). Dixon proposed an 8-way division of adjective types in English: dimension, physical property, colour, human propensity, age, value, speed, and position.

For this project, however, which has a smaller data set than either Ito \& 
Tagliamonte's (2003) York data set or Tagliamonte's (2008) Toronto one, and which is less interested in establishing trajectories of grammaticalisation, this categorisation is more fine-grained than necessary. A five-way division was adopted instead, and used for the initial analysis:

- abstract, e.g.: different, unattended, feminine, liberal, academic

- classification, e.g.: European, suburban, Catholic, rural

- concrete, e.g.: old, big, long, hot, quick, small, young

- emotional, e.g.: passionate, interested, angry, proud, nosy, keen, frustrated

- evaluative, e.g.: cool, great, good, top-notch, obnoxious, stupid, weird

For the narrower analysis of the intensified contexts only, where the total number of tokens was further reduced, a binary division was adopted (evaluative vs all others), as the majority of the intensified adjectives were evaluative, and this provided the most even split of the data.

\subsubsection{Evaluative orientation of the adjective}

Much of the previous work on intensifiers suggests that an important stage of grammaticalisation occurs when negative adverbs can be used to modify positive adjectives (e.g., terribly exciting, awfully good, etc.), and positive adverbs to modify negative adjectives (e.g., extraordinarily stupid, super useless, etc.) (e.g., Tagliamonte 2008; Bauer \& Bauer [2002] also note some interesting collocations with very and negative adjectives). I coded each token according to the inherent evaluative orientation of the adjective, based on its core meaning:

- positive, e.g.: good, nice, rewarding, lucky, prosperous

- neutral, e.g.: new, small, long, different, consistent

- negative, e.g.: weird, horrible, miserable, terrible, gross, nasty, painful

Previous studies (e.g., Ito \& Tagliamonte 2003; Tagliamonte \& Roberts 2005;

Tagliamonte 2008) have used a binary positive/negative distinction, without explicitly describing how those adjectives that I have classified as neutral were coded. Since there is nothing inherently good or bad about something being different or small, for example, I was not comfortable with arbitrarily classifying these adjectives as one or the other. The most workable compromise was to adopt a three-way categorisation scheme, and to classify adjectives that are not inherently positive or negative as neutral.

\subsubsection{Analysis}

The analysis and discussion that follow will focus on the effect of gender on adjectival modification, and will not explore the process of grammaticalisation in New Zealand English, which is beyond the scope of this project. Nevertheless, the linguistic factors described above are known to be relevant to the grammaticalisation (e.g., Ito \& Tagliamonte 2003) - and therefore also to the distribution - of intensifiers in other varieties, so they must be accounted for in the multivariate analysis if we want to be 


\begin{tabular}{|c|c|c|c|}
\hline & $\begin{array}{c}\text { overall } \\
\text { modification }\end{array}$ & $\begin{array}{c}\text { overall } \\
\text { intensification }\end{array}$ & $\begin{array}{c}\text { overall } \\
\text { moderation }\end{array}$ \\
\hline York $^{1}$ & & $24 \%$ & \\
\hline Toronto $^{2}$ & & $36.1 \%$ & \\
\hline Ottawa $^{3}$ & $36.3 \%$ & $23.5 \%$ & $12.8 \%$ \\
\hline Auckland & $27.3 \%$ & $20.7 \%$ & $6.6 \%$ \\
\hline
\end{tabular}

Table 4.3: Rates of modification in Auckland across age groups (raw Ns given in parentheses)

\begin{tabular}{lcc}
\hline & older & younger \\
\hline intensified & $20.2 \%(444)$ & $21.1 \%(651)$ \\
moderated & $6.3 \%(138)$ & $6.7 \%(208)$ \\
unmodified & $73.5 \%(1611)$ & $72.2 \%(2228)$ \\
\hline
\end{tabular}

\begin{tabular}{|c|c|c|c|}
\hline & unmodified & intensified & moderated \\
\hline oqm & 331 & 103 & 43 \\
\hline oqw & 237 & 79 & 14 \\
\hline osm & 423 & 92 & 34 \\
\hline osw & 372 & 116 & 32 \\
\hline otw & 248 & 54 & 15 \\
\hline yqm & 474 & 90 & 29 \\
\hline yqw & 326 & 80 & 37 \\
\hline ysm & 310 & 107 & 49 \\
\hline ysw & 484 & 165 & 40 \\
\hline ytm & 433 & 183 & 50 \\
\hline ytw & 201 & 26 & 3 \\
\hline
\end{tabular}


confident that the statistical model of the social constraints on variation produced is as accurate as possible. Multivariate analysis in sociolinguistics has gone through several iterations of variable rule analysis and regression modeling, discussed in $\S 3.6 .2$. In this analysis, we will focus on the role of the social factors within the larger system of structural variation, but note that the linguistic factors coded for have also been included in the statistical models that follow.

The first question we will address is the presence or absence of any kind of modification on the adjective. The second will focus on the choice of intensifiers by gendered identity group, and the third will look at the use of moderators across these same gendered identity groups; this will be limited to a descriptive examination rather than a statistical one, due to the low overall numbers of tokens.

\subsection{Intensifiers}

Table 4.2 gives the overall rates of intensification for this project [Auckland] as well as those reported for York, Toronto, and Ottawa, as points of comparison. It should be noted that the lower rates observed in Auckland compared with those in York and Toronto may be at least partly due to the exclusion of negative contexts in the previous studies, which would have the arithmetic effect of lowering the denominator, and thus raising the percentage. However, the Ottawa data was generated using a similar system of classification of negatives and litotes, so at least some of the difference observed between this study and that one is likely due to varietal differences.

\subsubsection{Overall adjectival modification}

In both the Toronto (Tagliamonte 2008) and the York studies (Ito \& Tagliamonte 2003), it was found that intensifier use was generally higher among younger participants. This does not appear to be the case in Auckland (Table 4.3), where the overall rates are nearly identical $(\dot{\chi}=0.6605, \mathrm{p}=0.719)$.

A more detailed breakdown of the rates of use is provided in Table 4.4, which shows the total numbers of tokens by gender group. Note that both older and younger trans women have substantially fewer tokens - modified and unmodified - than the other groups; these two groups are also the least-well-represented in the data, so the distribution is also provided in Figure 4.5, where it is presented as a percentage.

There are no clear social effects apparent in this distribution. There are no obvious clusters of groups according to age, gender, or identity, and the differences are generally quite gradient in any event - there is no marked division between groups at any point.

As this distributional data does not make any attempt to control for the effect of 


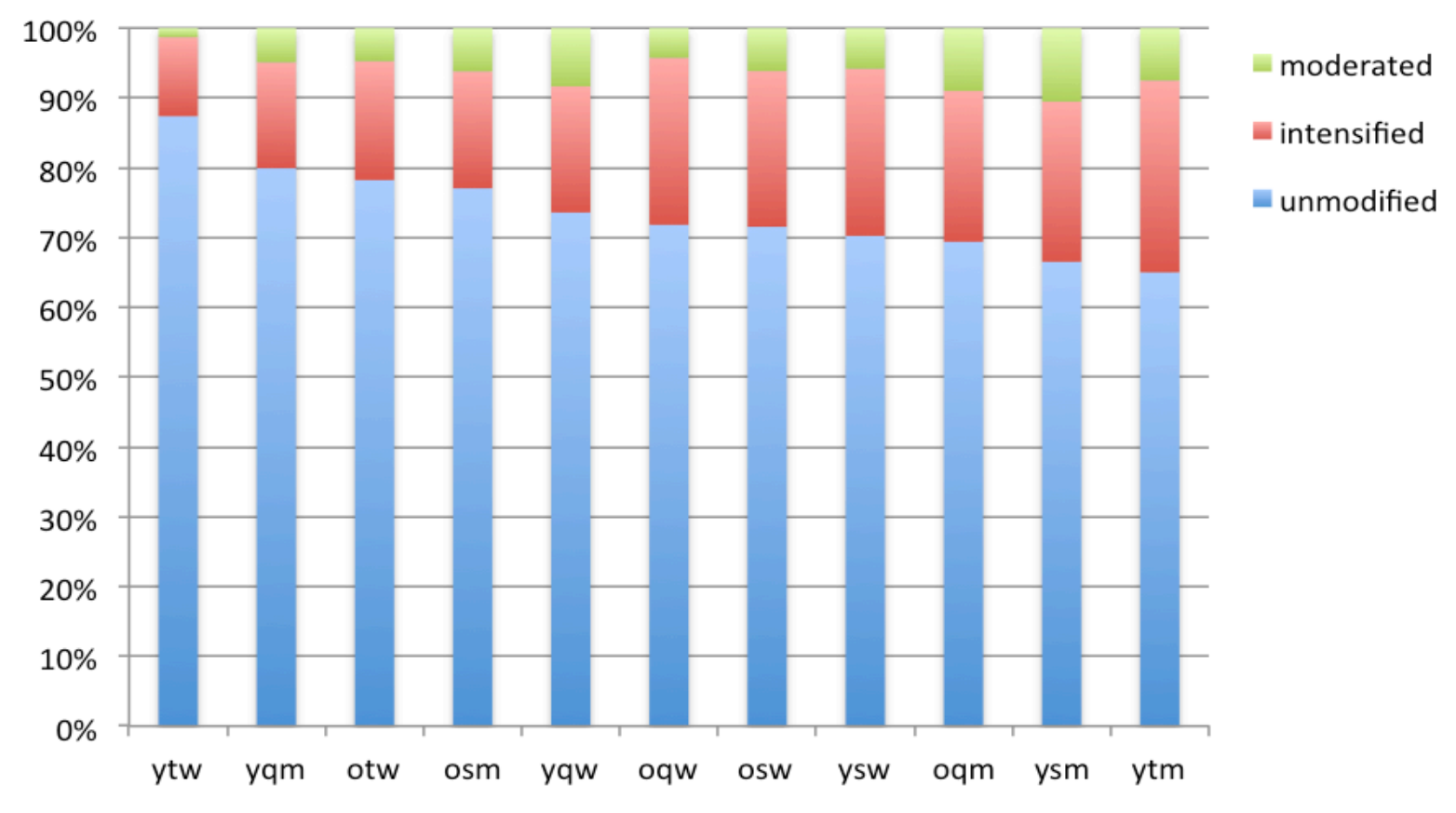

Figure 4.5: Adjectival modification by gender group (percentage)

\begin{tabular}{|c|c|c|c|}
\hline \multicolumn{4}{|c|}{$\begin{array}{l}\text { Table 4.5: Multivariate analysis of } \\
\text { modified vs unmodified adjectives - } \\
\text { social factors only (organised by age) }\end{array}$} \\
\hline input prob & 0.209 & & \\
\hline total $N$ & 5280 & & \\
\hline (older) & FW & $\mathrm{N}$ & $\%$ \\
\hline queer men & 0.593 & 146 & 31 \\
\hline straight women & 0.544 & 148 & 29 \\
\hline queer women & 0.526 & 93 & 28 \\
\hline straight men & 0.503 & 126 & 23 \\
\hline trans women & 0.422 & 69 & 22 \\
\hline \multicolumn{4}{|l|}{ range } \\
\hline (younger) & FW & $\mathrm{N}$ & $\%$ \\
\hline trans men & 0.606 & 233 & 35 \\
\hline straight men & 0.597 & 156 & 34 \\
\hline straight women & 0.521 & 205 & 30 \\
\hline queer women & 0.495 & 117 & 26 \\
\hline queer men & 0.405 & 119 & 20 \\
\hline trans women & 0.298 & 29 & 13 \\
\hline \multicolumn{4}{|c|}{$\begin{array}{ll}\text { range } & 0.308 \\
\end{array}$} \\
\hline \multicolumn{4}{|c|}{$\begin{array}{l}\text { Also retained as significant in model: } \\
\text { - adjective type (5-way classification) } \\
\text { - adjective evaluative orientation } \\
\text { (pos/neu/neg) }\end{array}$} \\
\hline
\end{tabular}




\begin{tabular}{|c|c|c|}
\hline \multicolumn{3}{|c|}{$\begin{array}{l}\text { Table 4.6: Most common intensifiers } \\
\text { (comprising at least } 5 \% \text { of the data) }\end{array}$} \\
\hline & $\mathrm{N}$ & $\%$ \\
\hline really & 244 & 22.3 \\
\hline very & 167 & 15.3 \\
\hline so & 142 & 13.0 \\
\hline quite & 91 & 8.3 \\
\hline just & 85 & 7.8 \\
\hline pretty & 62 & 5.7 \\
\hline all others & 304 & 27.8 \\
\hline \multicolumn{3}{|c|}{1095} \\
\hline
\end{tabular}

linguistic factors on the social ones, a multivariate analysis was also conducted (Table 4.5). This table has the gender groups organised by age for clarity, but the analysis itself was conducted with all eleven categories simultaneously.

This table shows that the decision to modify or not to modify an adjective is less indexical of gendered identity among older speakers than among younger ones, evidenced by the greater range in factor weights (FWs). However, it is worth noting that most of larger range is accounted for by the dispreference by younger trans women to any modification. The strongest favouring effect is shown in younger trans men, although this is not a particularly strong preference overall - FWs at 0.5 show no preference, and the strength of the effect increases with distance from 0.5 .

As noted previously, intensifiers are known to be a site for innovation and creativity, but this does not mean that there are no variants that occur with high enough regularity that they will have a coherent underlying system. In this data, the most common intensifiers were really, very, so, quite, just, and pretty (see Table 4.6).

Only three of these - really, very, and so - make up more than $10 \%$ of the data. This broadly agrees with previous findings, particularly Ito \& Tagliamonte (2003) in York. In the Canadian studies (Tagliamonte 2008; Brown 2009; Hazenberg 2012), pretty was also found to be fairly common, and particularly associated with younger (cissexual) men. Its absence in the list of most frequent intensifiers suggests that New Zealand English may be more similar to British English in this regard than to Canadian English.

\subsubsection{Distributional results}

Table 4.7 provides a breakdown of intensifier choice by gender group. Again, we see that trans women have very low token numbers across the different variants, making it difficult to draw any inferences about their preferences. It does seem, however, that both share a dispreference for quite.

Generally, really and very show fairly consistent distribution, although there are some trends. Older queer and straight men use very more than really, while older queer 


\begin{tabular}{|c|c|c|c|c|c|c|c|c|}
\hline & really & very & so & quite & just & pretty & other & total \\
\hline oqm & $11.7(12)$ & $33.0(34)$ & $6.8(7)$ & $12.6(13)$ & $12.6(13)$ & $3.9(4)$ & $19.4(20)$ & 103 \\
\hline oqw & $39.2(31)$ & $15.2(12)$ & $10.1(8)$ & $7.6(6)$ & $8.9(7)$ & $2.5(2)$ & $16.5(13)$ & 79 \\
\hline osm & $6.5(6)$ & 20.7 (19) & $4.3(4)$ & $13.0(12)$ & $12.0(11)$ & $4.3(4)$ & $39.1(36)$ & 92 \\
\hline osw & $24.1(28)$ & $18.1(21)$ & $13.8(16)$ & $10.3(12)$ & $13.8(16)$ & $0.9(1)$ & $19.0(22)$ & 116 \\
\hline otw & $14.8(8)$ & $16.7(9)$ & $9.3(5)$ & $0.0(0)$ & $3.7(2)$ & $3.7(2)$ & $51.9(28)$ & 54 \\
\hline yqm & $22.2(20)$ & $24.4(22)$ & $10.0(9)$ & $6.7(6)$ & $4.4(4)$ & $8.9(8)$ & $23.3(21)$ & 90 \\
\hline yqw & 17.5 (14) & $10.0(8)$ & $21.3(17)$ & $12.5(10)$ & $6.3(5)$ & $2.5(2)$ & $30.0(24)$ & 80 \\
\hline ysm & $41.1(44)$ & $11.2(12)$ & $6.5(7)$ & $9.3(10)$ & $0.9(1)$ & 3.7 (4) & $27.1(29)$ & 107 \\
\hline ysw & $18.2(30)$ & 7.9 (13) & $21.8(36)$ & $4.8(8)$ & 7.3 (12) & 7.9 (13) & $32.1(53)$ & 165 \\
\hline ytm & $23.0(42)$ & 7.1 (13) & $16.4(30)$ & 7.7 (14) & $5.5(10)$ & $10.9(20)$ & $29.5(54)$ & 183 \\
\hline ytw & $34.6(9)$ & 15.4 (4) & $11.5(3)$ & $0.0(0)$ & 15.4 (4) & 7.7 (2) & 15.4 (4) & 26 \\
\hline total & 244 & 167 & 142 & 91 & 85 & 62 & 304 & 1095 \\
\hline
\end{tabular}

and straight women prefer really; older trans women show no marked preference for either. Younger speakers on the whole prefer really, and younger straight men prefer it by the widest margin. This preference for really among younger speakers (except for younger queer men, who show a slight preference for very) is consistent with Hay, Maclagan \& Gordon's (2008: 62) observation that very is in decline in NZE, although it is clearly still used by younger speakers. Recall that there are methodological and demographic differences between the ATC and the corpora used in previous studies, so this finding of the increasing use of very at the cost of really may not actually indicate regional stratification in New Zealand, but may rather be a product of these methodological differences.

The rates of use for so are generally higher among younger speaker than older ones, which supports so as a newer variant in NZE, as it is in other varieties of English. So shows the highest rates of use among younger straight and queer women, which suggests that it is gendered; the two groups with the lowest rates of so use are older and younger straight men. The next highest-using group is younger trans men, for whom it is the second-most-preferred variant, after really, while among other groups of men, it is generally low. Younger trans women are low- to moderate-users of so, although given their overall very low rate of intensification, it is difficult to extrapolate much from this observation.

Pretty, which is generally not used as an intensifier to any great degree, is more preferred by younger trans men, and next by younger straight women. This shows not only much lower rates of use than so, but also a less marked gender profile. In Canadian English in particular, pretty is associated with younger cissexual men; clearly, in NZE, this is not the case. Scandrett (2012: 40-41) shows generally low and stable rates of use of pretty by women across a time depth of 120 years, while the rates of use among men 
over this same time depth show more variation, peaking at between $30 \%$ and $40 \%$ for men born in the 1950s and 1960s. This corresponds with the older men in the ATC, although we do not see any such spike in usage among the older ATC men, so this may be a regional or a methodological point of difference between this study and those that came previously.

Quite shows marginally higher rates of use among older speakers than younger ones, as does just, although the overall number of tokens is low across these two variants so it is inadvisable to read much socially into this distribution. Neither Scandrett (2012) nor Saarenpää (2016) appear to have classed quite and just as intensifiers, and so there is no data with which to compare these rates of use.

With regards to gender, then, we have seen a divided preference pattern among older speakers for really (preferred by cis women) and very (preferred by cis men), and higher rates of use of so among younger cis women. Pretty, gendered in Canadian English, does not seem to be a productive part of the gendered system in NZE.

\subsubsection{Statistical models of intensifier choice}

We turn now to the multivariate analysis of the intensifiers. Because this statistical approach aims to balance the demands of multiple factors simultaneously, and to assign a factor weight which correlates roughly with the probability of the response variable occurring with a particular factor, extremely skewed data (e.g., cells with zero or only one or two tokens) can have a disproportionately large effect on the overall models. It is generally more reliable when working with larger numbers of application tokens, so we will limit our analysis to really, very, and so.

When working with categorical variables, Rbrul limits its analysis to a binary case: application vs non-application. With that in mind, the following analyses each test one variant against all other intensifiers, but exclude non-intensified contexts, effectively asking: If a speaker chooses to use an intensifier, what constrains or favours the choice of really vs very vs so? A statistical model was generated with gendered identity in its eleven-category organisation, and a separate model was generated with these eleven categories broken down into their component parts (age, identity, and gender); the models with the best fit (ascertained through a log-likelihood test in Rbrul) are the ones reported on here. Linguistic constraints included in the model were the two-way classification of the adjective as evaluative or non-evaluative, the evaluative polarity of the adjective, and the syntactic position of the adjective. Speaker is included as a random effect.

\subsubsection{Really}

As Table 4.8 shows, the better model with really is the one that retains the eleven-way split in the gendered identity factor group. The factor weights are organised by age group in this table, but only for the sake of clarity: gendered patterns are more evident when we 


\begin{tabular}{|c|c|c|c|}
\hline \multicolumn{4}{|c|}{$\begin{array}{l}\text { Table 4.8: REALLY versus all other } \\
\text { intensifiers - social factors only } \\
\text { (organised by age) }\end{array}$} \\
\hline input prob & 0.192 & & \\
\hline total $N$ & 1095 & & \\
\hline (older) & FW & $\mathrm{N}$ & $\%$ \\
\hline queer women & 0.706 & 31 & 39 \\
\hline straight women & 0.545 & 28 & 24 \\
\hline trans women & 0.371 & 8 & 15 \\
\hline queer men & 0.338 & 12 & 12 \\
\hline straight men & 0.200 & 6 & 7 \\
\hline \multicolumn{4}{|c|}{0.506} \\
\hline (younger) & FW & $\mathrm{N}$ & $\%$ \\
\hline straight men & 0.703 & 44 & 41 \\
\hline trans women & 0.670 & 9 & 35 \\
\hline trans men & 0.541 & 42 & 23 \\
\hline queer men & 0.518 & 20 & 22 \\
\hline queer women & 0.466 & 14 & 18 \\
\hline straight women & 0.466 & 30 & 18 \\
\hline \multicolumn{4}{|c|}{$\begin{array}{ll}\text { range } & 0.237 \\
\end{array}$} \\
\hline \multicolumn{4}{|c|}{$\begin{array}{l}\text { Also retained as significant in model: } \\
\text { - adjective evaluative orientation } \\
\text { (pos/neu/neg) }\end{array}$} \\
\hline
\end{tabular}

consider them within peer groups. The model considered all gendered identity categories simultaneously, however; note also that the adjective's evaluative orientation was retained as a significant predictor.

Among older speakers, we see that really is strongly favoured by queer women, that straight women neither strongly favour nor disfavour it, and that the remaining groups - presumably all using really as they learned to early in life - show a moderate to strong disfavouring effect. The use of really among older speakers may be indexical of a particular style of femininity, and its avoidance is indexical of a particular kind of masculinity; the moderate disfavouring effect observed among trans women here would then suggest that either the preference for really among older women is under the radar in the speech community (with trans women retaining the system they acquired early in life) or that it is indexing a particular style of femininity that they are distancing themselves from.

Among the younger speakers, we see that the use of really is favoured quite strongly by younger straight men and by younger trans women, and is only weakly favoured or disfavoured by the remaining groups. Use of really in this age group may then index a form of masculinity, although we do not see the same magnitude of avoidance on the part of young women that we saw with older men. The trans men in 
this age group are patterning more like queer men than they are like either queer or straight women, although because the range of factor weights is quite small between them, it is not clear with how much confidence we should read social meaning into this distribution. The close parallel between straight men and trans women is an interesting pattern, as we would not predict these young women to be 'doing' masculinity. Recall, however, that these young women are both Pasifika, and may be orienting to a different set of norms overall - and in fact, we will see this convergence again with other variables as well; a potential indexical chain for this unexpected similarity is discussed in §7.4.4.

So for older speakers, then, really may be indexing some type of femininity, while encoding masculinity for younger speakers. This suggests that, although overall rates of use of intensifiers do not seem to be changing much, the distribution of particular intensifiers has undergone, or is currently undergoing, some degree of reshuffling with respect to social meaning. This data also shows that really is more strongly gendered among older speakers than among younger ones, as we can see by the difference in magnitude of the range for both age groups. The change between the two age groups is perhaps best characterised, then, as a weak neutralisation of the gender-indexicality handin-hand with a reorganisation of that same indexicality.

\subsubsection{Very}

As with really in the previous section, the data presented in Table 4.9 has been organised by age group for clarity, but the analysis was conducted with all speaker groups simultaneously. The best model was built using the eleven-way gender categorisation, and also retained the adjective's evaluative orientation as a significant predictor.

We see a very broad reversal of the pattern that we observed with really, in that younger speakers show a more exaggerated gender effect than older speakers. This gender effect is smaller than the one we observed with older speakers for really, but the fact that really carries more gendered meaning for older speakers, and very for younger ones, is interesting. Really is the more recent intensifier of the two, so we might expect to see more of a difference with really among younger speakers. However, both really and very have been productive intensifiers in English since the $18^{\text {in }}$ century, so although there may be fine-grained differences at the structural level (e.g., marked and unmarked collocations), we would not necessarily expect to find social differences at this stage of grammaticalisation.

Among both age groups, the strongest favouring effect is observed in queer men, suggesting that very carries an association with a particular style of masculinity. The difference in FWs between queer and straight men is larger in the younger cohort than in the older one, which implies that this indexicality of queer masculinity is an innovation that is continuing to propagate through the speech community. The association with a queer masculinity is stronger among younger speakers than it is among older ones, given that older straight men very slightly favour the use of very while younger straight men 


\begin{tabular}{|c|c|c|c|}
\hline \multicolumn{4}{|c|}{$\begin{array}{l}\text { Table 4.9: VERY versus all other } \\
\text { intensifiers (organised by age) }\end{array}$} \\
\hline input prob & 0.126 & & \\
\hline total $N$ & 1095 & & \\
\hline (older) & FW & $\mathrm{N}$ & $\%$ \\
\hline queer men & 0.750 & 34 & 33 \\
\hline straight men & 0.595 & 19 & 21 \\
\hline trans women & 0.551 & 9 & 17 \\
\hline straight women & 0.544 & 21 & 18 \\
\hline queer women & 0.508 & 12 & 15 \\
\hline \multicolumn{4}{|l|}{ range } \\
\hline (younger) & $\mathrm{FW}$ & $\mathrm{N}$ & $\%$ \\
\hline queer men & 0.671 & 22 & 24 \\
\hline trans women & 0.482 & 4 & 15 \\
\hline straight men & 0.395 & 12 & 11 \\
\hline queer women & 0.366 & 8 & 10 \\
\hline straight women & 0.324 & 13 & 8 \\
\hline trans men & 0.306 & 13 & 7 \\
\hline \multicolumn{4}{|c|}{$\begin{array}{ll}\text { range } & 0.365\end{array}$} \\
\hline \multicolumn{4}{|c|}{$\begin{array}{l}\text { Also retained as significant in model: } \\
\text { - adjective evaluative orientation } \\
\text { (post/neu/neg) }\end{array}$} \\
\hline
\end{tabular}

disfavour it. This difference is interesting: as we will see when we look across other variables (sibilants in chapter five and vowels in chapter six), linguistic disaffiliation between queer and straight men tends to be more pronounced among older speakers than younger ones. There may be a fundamental difference, then, in the perception of lexical variables versus phonetic ones: phonetic variables may be more about sounding queer, while lexical variables such as intensifier choice may be more about being queer. At this point, this is a very tentative observation, and we will return to it after we have examined the phonetic variables in question.

\subsubsection{So}

As it turns out, social effects are not retained as significant with so, which means that the trends that we observed in the previous section, although suggestive, are not strong enough to exert an influence in the face of linguistic constraints. We will therefore not discuss so further in this thesis, but we will note that a similarly-designed study with a larger data set of intensifiers may find in the future that social effects are retained as significant. 


\subsection{Moderators}

Quantitative studies of variation in the system of adjectival moderators and downtoners are not as common as those of intensifiers. This is partly because, as noted previously, studies that have looked at them have tended not to find much by way of interesting variation, and so they are not often replicated. It may also be partly because moderators do not occur as frequently as intensifiers. This is not to say that moderation (or hedging) is on the whole infrequent, but only that the role of moderators as modifiers of adjectival heads is not as common; a study looking at moderation of verbs and sentences would almost certainly have a much bigger pool of data to draw from.

\begin{tabular}{lcc}
\hline \multicolumn{3}{l}{ Table 4.10: } \\
\multicolumn{3}{c}{ (comprising at least } \\
\hline \multicolumn{2}{c}{ 5\% of the data) } \\
\hline kind of/sort of & $\mathrm{N}$ & $\%$ \\
quite & 68 & 19.9 \\
a (little) bit & 56 & 19.6 \\
litotes & 50 & 16.2 \\
enough & 29 & 14.4 \\
all others & 74 & 21.4 \\
\hline \multicolumn{2}{c}{ total } & 346 \\
\hline
\end{tabular}

However, as this chapter is looking at modification of adjective heads in spontaneous speech data, we will restrict ourselves to this smaller data set, and to the limitations this places on the type of analysis possible. The overall rate of modification in this data set is $6.6 \%$ (of a total of 5280 modifiable adjectives), and even when we restrict ourselves to the adjectives that have been modified in some way (1441), only $23.1 \%$ of these have been moderated as opposed to intensified, yielding a total of 346 tokens for examination. In other words, intensifiers are used around three times as frequently as moderators. Table 4.10 summarises the most commonly-occurring moderators in this data set.

The most commonly occurring variants are kind of/sort of, quite, a (little) bit, litotes, and enough. Two of these categories are composites: kind of/sort of includes all tokens of both kind of and sort of, including their reduced forms kinda and sorta; and $a$ (little) bit includes a little, a bit, and a little bit. These were grouped together mainly so that there would be categories big enough to talk about meaningfully, and also because of the strong semantic and collocational overlap between the constituent categories.

\subsubsection{Distribution}

Table 4.11 presents the most common moderators across the gendered identity 


\begin{tabular}{|c|c|c|c|c|c|c|c|}
\hline & $\begin{array}{l}\text { kind of/ } \\
\text { sort of }\end{array}$ & quite & $\begin{array}{c}a \text { (little) } \\
\text { bit }\end{array}$ & litotes & enough & other & total \\
\hline oqm & $9.3(4)$ & $32.6(14)$ & $23.3(10)$ & $7.0(3)$ & $7.0(3)$ & 20.9 (9) & 43 \\
\hline oqw & $7.1(1)$ & $42.9(6)$ & $35.7(5)$ & $7.1(1)$ & $7.1(1)$ & $0.0(0)$ & 14 \\
\hline osm & $5.9(2)$ & $2.9(1)$ & $14.7(5)$ & $29.4(10)$ & $8.8(3)$ & $38.2(13)$ & 34 \\
\hline osw & $34.4(11)$ & $25.0(8)$ & $25.0(8)$ & $3.1(1)$ & $0.0(0)$ & 12.5 (4) & 32 \\
\hline otw & $6.7(1)$ & $13.3(2)$ & $13.3(2)$ & 20.0 (3) & $13.3(2)$ & $33.3(5)$ & 15 \\
\hline yqm & 24.1 (7) & $6.9(2)$ & $17.2(5)$ & $10.3(3)$ & $13.8(4)$ & $27.6(8)$ & 29 \\
\hline yqw & $13.5(5)$ & $13.5(5)$ & $10.8(4)$ & $37.8(14)$ & 10.8 (4) & $13.5(5)$ & 37 \\
\hline ysm & $24.5(12)$ & $18.4(9)$ & $8.2(4)$ & $14.3(7)$ & $10.2(5)$ & $24.5(12)$ & 49 \\
\hline ysw & $17.5(7)$ & 22.5 (9) & $15.0(6)$ & $5.0(2)$ & $7.5(3)$ & 32.5 (13) & 40 \\
\hline ytm & $38.0(19)$ & $22.0(11)$ & $10.0(5)$ & $12.0(6)$ & $8.0(4)$ & $10.0(5)$ & 50 \\
\hline ytw & $0.0(0)$ & $33.3(1)$ & 66.7 (2) & $0.0(0)$ & $0.0(0)$ & $0.0(0)$ & 3 \\
\hline total & 69 & 68 & 56 & 50 & 29 & 74 & 346 \\
\hline
\end{tabular}

categories of our participants. We see that the distribution of variants across the different groups has generally very low numbers, including several singletons $(\mathrm{N}=1)$ and knockouts $(\mathrm{N}=0)$ making it difficult to draw meaningful inferences, or indeed to do any kind of statistical examination of the distribution. However, we can make a few tentative observations, at least for some of the variants, although clearly we are limited in how generalisable these observations are.

\subsubsection{Kind of/ sort of}

Kind of/sort of is the most commonly used moderator, closely followed by quite, and these two variants at least suggest some different preferences by the various speaker groups. Among older speakers, kind of/sort of is used most by straight women, for whom it is also the most frequently used variant. In the younger age cohort, it is used most by trans men, followed by straight men and queer men, for each of whom it is also the most frequently used variant.

Similar to what we saw with really, there is a gender reversal between the two age cohorts, where among younger speakers kind of/sort of seems associated with masculinity, while in older speakers it is associated with a particular style of femininity. Without enough data to conduct a more structural analysis, we can only tentatively suggest that for young men, kind of/sort of may carry an overtone of indifference or distance, while for older straight women it may be more of a generic hedging strategy.

\subsubsection{Quite}

Quite has an overall comparable rate of use as kind of/sort of, but is preferred by different groups of speakers. Among older speakers, it is most commonly used by queer women and queer men, for whom it is the preferred variant. Among the younger speakers, the 
highest rates of use are by straight women and trans men; note that trans women have only three tokens of moderators in total, so it is perhaps better to make no inferences at all about their choices of moderator. For younger straight women, quite is the preferred variant, while for trans men it is the second most popular choice. For older speakers, then, it may be part and parcel of a queer identity, while among younger speakers it is associated with a particular style of femininity.

\subsubsection{Litotes}

Although the overall number of litotes tokens is comparable to that for a (little) bit, the distribution is much less even across the groups of speakers. Among older speakers, litotes is most commonly used by straight men, for whom it is also the most common variant, followed by older trans women, although it is also advisable not to read too much into this distribution due to the overall low numbers. Among younger speakers, the most common users are younger queer women, for whom it is also the majority variant.

Recall that litotes, unlike the other variants, is not one fixed word or expression, but is rather a rhetorical strategy of understatement through negation, and so may reflect more of an interactional stance than any of the other variants, for example not committing to extremes - It's not bad as opposed to It's wonderful - or a distancing kind of irony. A wider study of the use of litotes in New Zealand English would be an interesting undertaking, as the strategy can be used with other syntactic units as well as with adjectives (e.g., The campaign wasn't a total success; It might not be the best idea).

\subsubsection{A (little) bit and enough}

Given the overall low numbers of moderators in this data, a (little) bit is relatively balanced across the gender groups, with no knockouts $(\mathrm{N}=0)$ or singletons $(\mathrm{N}=1)$. It shows a slightly higher use among older queer men, but with such small numbers generally, it is difficult to read much significance into this.

Like a (little) bit, enough shows a fairly uniform distribution across the speaker groups, although again, the numbers are too low to extrapolate anything meaningful.

\subsection{Trans speakers}

With the multivariate analyses in $\S 4.3 .3$ on intensifiers, as well as in the distributional discussion in section $\$ 4.4 .1$ on moderators, we noted some gendered differences. With the intensifiers, we considered the possiblity that use of really carries an indexicality of femininity in older speakers and masculinity in younger speakers, and that use of very is preferred by queer men across both age groups. With the moderators, we saw that kind of/sort of may index straight femininity in older speakers and a style of queer masculinity in younger speakers. We also noted that quite seems associated with older queer 


\begin{tabular}{c|ccc|ccc}
\hline \multicolumn{3}{c}{ Table 4.12: } & Similarities in modifier choice for trans speakers \\
\hline \multirow{2}{*}{$\begin{array}{c}\text { multivariate analysis } \\
\text { overall }\end{array}$} & really & very & \multicolumn{3}{c}{ distributional trends } \\
& kodification & & & sort of & quite & litotes \\
\hline otw & osm & oqm & osw & oqw & osm & osm \\
ytm & ysm & yqm & ysw & ysm & ysw & ysm \\
ytw & yqm & ysm & ysm & [ ] & [ ] & [ ] \\
\hline
\end{tabular}

speakers and younger straight women, and that litotes is used most by older straight men and younger queer women.

But to what extent do we see trans speakers mirroring the gendered patterns in the broader speech community? Table 4.12 provides a brief summary of how the trans participants in this study pattern with respect to the other gendered groups in their age cohorts, for the overall rates of adjectival modification as well as for the specific effects noted with particular variants. For the intensifiers, we are looking at the patterning retained as significant in the multivariate analysis, while with the moderators we only have the raw distributional trends as evidence. Note also that younger trans women are excluded from this comparison with respect to the moderators, as they have overall too few tokens to confidently note any trends.

For older trans women, we see a mix in similarities. Their use of very is closest to that of older straight women, but their intensification otherwise is closest to men. With the moderators, they use kind of/sort of on par with queer women, but for both quite and litotes they are more like straight men. This suggests that, within the system of adjectival modification among older speakers, very and kind of/sort of are the most salient with respect to gender, in the sense that these are the variants with which trans women more closely approximate the cis speakers of their identified gender. That is to say, very is the intensifier that indexes femininity more than the alternatives, and kind of/sort of is the moderator that does likewise. Taking a similar approach with the younger trans men, we would argue that for younger speakers, really, kind of/sort of and litotes are the variables that index gendered identity, but that quite and very are less relevant.

The data from the younger trans women seems to contradict this pattern, but as noted above, these women are both Pasifika, and may in fact be orienting to a generally different system of adjectival modification, as part of a South Auckland or Pasifika variety of New Zealand English (see §2.3.2.3 and §7.4.4).

These trends based on the older trans women and the younger trans men are counter-intuitive in a sense: the multivariate analyses suggested that really had a greater gendered effect among older speakers, and very among younger speakers. However, this perspective - of examining the practices of trans speakers as a window on how perceptually salient these variables are - is not exactly the same as the question of production data. Neither is more empirically correct than the other; the production data, at least for really and very where we had enough tokens to conduct a multivariate 
analysis, are indicative of what people are doing. The inferences we can draw based on what trans speakers are doing are around the extent to which they are (consciously or unconsciously) aware of the patterns in the production data. So although really may be used differentially to a greater extent among older speakers, its relevance as a social marker of gender may be more salient to younger speakers. What motivates this mismatch is a different question, and not one that this data is positioned to answer, unfortunately.

Although the inconsistency across the variants could also be taken as evidence of an absence of pattern overall - i.e., that there is no gendered indexicality at play in the system of adjectival modification in New Zealand English - we might then wonder why trans participants are patterning with their identified gender for some of the variants, but not others. If there is no social meaning, why not stick to the system they would have acquired as children? The fact that some intensifiers seem to do quite a lot of gendered work in other varieties of English (i.e., the Canadian and UK varieties discussed in $\$ 4.1 .4$ above) means that it is not unreasonable to assume that something similar may be happening in New Zealand; that is to say, intensifiers in English clearly can support gendered presentation, and there is no reason to think that New Zealand English is exceptional in this regard. While it is not entirely clear why very in particular should signal femininity, for example, there is also no compelling reason why it should not. The gendered associations that we see with kind of/sort of may be reflective of the stereotype that certain women use more hedging generally; that is to say, kind of/sort of moderates adjectives in the same way that it can be used to hedge other propositions (e.g., I kind of want to see that movie), which may in turn index older straight women, but not queer or trans women who are distancing themselves from that hesitant or overly-accommodating style of femininity. The preference for kind of/sort of among younger men may index an indifferent, non-committal stance that is associated with a particular style of masculinity.

It is worth noting that phonetic studies of variation across multiple variables, such as the Atlas of North American English (Labov, Ash \& Boberg 2006) and the Accent and Identity on the Scottish/English Border project (e.g., Llamas 2012; Llamas, Watt \& MacFarlane 2016), highlight the fact that critical differences between regional or national dialects are often down to one or two realisations (e.g., a particular vowel contrast, or rhoticity) within a larger set of variables. Although this project is looking at gendered rather than regional variation, and the variable under discussion in this chapter is a lexical rather than a phonetic one, the underlying mechanism of differentiation may be comparable. That is to say, the critical salient difference between contrasting styles of masculinity and femininity may be relatively small and discontinuous compared to larger patterns of convergence, and still be socially meaningful. So while the evidence for gendered indexicality in this particular case is slim, resting as it does on a very small number of variants within a larger system, this does necessarily rule out the salience of these particular variants. 


\subsection{Discussion}

There are several interesting aspects of this study of intensifiers and moderators in Auckland English, particularly when compared with some of the previous variationist studies.

\subsubsection{Relative (structural) stability of the system}

Although this study did not focus on all of the results of the full multivariate analysis conducted here, relevant linguistic constraints were included in the statistical model as well as the social ones of interest. While a full a discussion of these structural constraints is beyond the scope of this project, it is worth mentioning that in general, the models produced in this study are simpler than those produced in York and Toronto.

This could be a result of having a smaller data set, or the larger dimensions of the gendered space (eleven categories rather than the more traditional two) and the effect that has on the statistical model. Alternatively, it could be that the system of intensifiers in NZE is not undergoing as rapid a change as that observed in York and Toronto. Recall that age was not a clear factor in the overall rates of intensification, which does suggest a more stable system overall; although age was helpful to our interpretation of the patterns in the data, it does not necessarily mean that age is a relevant predictor of structural variation in the system of intensifiers. Recall that the best statistical models were the ones that used the composite age+identity+gender construct, rather than the ones that looked at decomposed gendered identity (i.e., age, identity, and gender as separate categories).

We saw what appeared to be an age-related reversal of preference between really and very, with really showing a stronger gender effect among older speakers, and very among younger ones. On the one hand, this type of age effect looks like a straightforward change in progress, with younger speakers showing a preference for the incoming variant. On the other hand, it is hard to argue that very is an incoming variant, given that its use as an English intensifier has been documented as early as the $16^{\text {th }}$ century (Mustanoja 1960: 319-328, cited in Ito \& Tagliamonte 2003: 260). But it is important to remember that studies looking at trajectories of grammaticalisation are only paying secondary attention at best to the social aspects of variation. That is to say, they are interested in what social variation tells us about grammaticalisation: if we know that $\mathrm{X}$ is an innovation, we would expect younger speakers - and younger women in particular - to be using it more frequently; and conversely, if we know that the system is undergoing change and we see that younger women are using $\mathrm{Y}$ more than anyone else, then it follows that $\mathrm{Y}$ is a likely candidate for the incoming form.

Neither of these scenarios fits the patterns that we see here particularly well, so we may need to look elsewhere for an explanation. One possibility is that, given really and very are well-established in the contemporary system of English intensifiers, then 
what we are seeing is variation in the indexical field of the system, rather than in the system itself. An example of what this might look like is perhaps best sourced from outside of linguistics, so we will draw on Eckert's $(2000 ; 2008)$ discussion of clothing as stylistic bricolage in her work with high school students in California. Eckert describes the process by which two girls in a popular, school-oriented and conformist clique adapt their style in a deliberate move to affiliate with certain aspects of a countercultural group within the school:

"they segmented the new wave style into meaningful elements, most saliently cut and colour, identified the cut of one's jeans as indexing autonomy but not rebellion or sluttiness, and worked it into their otherwise preppy style, ultimately making a claim to being both preppy and independent" (Eckert 2008: 457)

Here, the cut of the jeans (pegged vs straight-legged) is analogous to a linguistic variable, used widely with one group of speakers (or, in this case, dressers), recognised as a resource by another group, and appropriated by this second group for their own purposes. The preppy girls are not proclaiming themselves suddenly as new-wavers, but they are indexing part of what it means to be new wave, and incorporating it into their own style. The relationship between fashion and linguistic practice is not as discontinuous as one might first think, and it is important to note that Eckert herself incorporates this discussion of fashion indexicality into a theoretical framework for understanding sociolinguistic variation.

Of course, this thesis is not addressing fashion, or indeed any of the other dozens of ways that individuals perform their identities, but the parallel with Eckert's example of fashion choice is an apt one, as it shows the way that the meaning of a variable (whether clothing or language) can be re-interpreted and re-inscribed with a different set of social meanings. The mechanisms for shifts in the linguistic indexical field are not well understood, but it is not unreasonable to assume that they will be qualitatively different from those in the semantic and syntactic fields, so we should not expect the patterns of variation in indexicality to be the same as those in structural domains.

This data does not explore any evidence of syntactic and structural changes that have been observed in intensifiers in other varieties of English. However, it does show evidence of some kind of change, and that change seems largely to be social - which points to the indexical field as the relevant domain. Intensifiers in New Zealand English may be syntactically relatively stable, but this does not rule out the possibility of other types of changes being underway.

\subsubsection{Age as a perceptual lens}

With both really and very, we found it useful to separate out the older speakers from the younger ones. We did not do this to influence the outcome of the analysis, since we did this after the statistical model had been generated, but rather to help drive 
meaning in our interpretation of the data.

Age is a relevant and visible dimension to the organisation of New Zealand society, so it makes sense that age is one of the filters through which we perceive the social world around us. There are, for example, fashion trends that are widely popular across the younger sectors of society, but which would look decidedly out of place if worn by older people. Similarly, there are musical tastes that are taken for granted of older people which would be either strange or self-consciously ironic if they were expressed by youth. It follows, then, that we use age as a filter when making sense of the spoken world that we encounter, and evaluate what people say - and how they say it - in light of how we perceive their age. It also follows that speakers are likely aligning themselves with an age-appropriate gendered group, particularly given the changes in social climate that have occurred in New Zealand between the formative years for our older speakers and our younger ones (discussed in \$2.2). That age-appropriate group may be defined in relation to, or in opposition to, some other group, as we saw with reinterpretation of very, from a sexuality-neutral [+masculine] in older speakers to [+ queer masculine] in younger ones.

\subsubsection{Intensifiers as a gendered resource}

On the whole, adjectival modification does not seem to be as broadly gendered in New Zealand English as it is in Canadian or UK varieties, in that there are no variants that are as strongly associated with gendered identity as have been found elsewhere. However, we did see that there are general patterns of usage for really and very that correlate with particular styles of masculinity and femininity, and that these associations are not consistent across age groups.

We have also seen that the linguistic behaviour of our trans participants does not always closely align with the patterns observed in the cissexual participants, which suggests that there is a mismatch between the production and perception ends of the system of adjective modifiers. This is not to say that there aren't differences in how people use intensifiers - we have seen that such differences clearly do exist. There are variants that seem particularly to index certain identities, and these variants are quite strongly disfavoured by other groups of speakers; the mismatch is in which variants seem relevant for trans speakers. The fact that trans speakers seem to be making strategic use of intensifiers, whether consciously or unconsciously, suggests that the system of adjectival modification as a whole is a resource for gendered identity in the speech community generally. Note that, without a multivariate analysis of moderators, it is hard to argue the same point with any great conviction, because as we saw in the case of so, gendered distributional patterns may disappear when other factors are taken into consideration.

One of the ways that linguists have tended to address differences in perceptual salience is to talk about variables as being above or below the radar (e.g., Eckert 2008). 
Variables that are above the radar are accessible to overt commentary, stereotyping, and stigmatisation, while those that are below the radar are not; this corresponds roughly to whether or not members of a speech community are consciously aware of a given linguistic feature (cf. §1.2.2.1). This distinction is often made based on the types of metalinguistic and metapragmatic commentary that circulates in the speech community: if everyone knows about $\mathrm{X}$, and they have an opinion on the types of people who do or don't do X, then it is obviously above the radar. If a variable goes overtly unnoticed, and is uncommented-on, then it is thought to be below the radar.

But the inclusion of trans people in linguistic study, particularly if we are interested in their attention to variation in the speech community, complicates this model somewhat, in that they may be very attuned to, and make performative use of, variables that they are not consciously aware of manipulating. If this is the case, then we need a different taxonomy of linguistic variables, to distinguish between those that carry conscious social meaning, those that carry unconscious social meaning, and those that do not carry social meaning at all. We will return to this point in $§ 7.6$, when we begin to assemble the methodological approach and theoretical consequences of including liminal identities in sociolinguistic research. First, however, we will look at variation at the phonetic level. 


\section{Chapter five: Sibilants}

\subsection{Introduction}

As a class of sounds, fricatives have received a considerable amount of linguistic attention, both cross-linguistically and within specific languages. Catford (1968: 322) describes fricatives as involving "the formation of an articulatory channel of such small cross-sectional area (of the order of 4 to $30 \mathrm{~mm}^{2}$ ) that at normal operating volume-flows (say 150 to $200 \mathrm{~cm}^{3} / \mathrm{sec}$ ) the airflow through the channel is always turbulent". This combination of small cross-sectional area and high rate of flow results in a particularly noisy sound, which is characterised in spectrogram analysis as having an aperiodic band of energy expenditure across a particular range of frequencies. One particularly interesting finding has been that, for fricatives whose constriction is inside the mouth, there is a direct relationship between the peak frequency observed in a spectral analysis of the segment and the place of articulation (e.g., Stevens 1989, cited in Johnson 2003: 126). A higher peak frequency corresponds to a fronter articulation, while a lower peak frequency corresponds to a more retracted articulation.

Figure 5.1 shows two spectrograms of short speech samples from Caroline, a 25-year-old straight woman. There are four fricatives represented across these two samples: [s] in stuff, [f] in stuff, [ $\theta]$ in north and [J] in shore. The vertical axes of these two spectrograms, which show frequency, are on the same scale in both images, and several of the properties of fricatives can be observed here.

First, we can see that fricatives are aperiodic, not showing the evenly spaced vertical lines characteristic of vowels. These bands represent of the vocal fold vibrations, recurring at the fundamental frequency (F0) of the speaker, and are particularly clear in resonant sounds such as vowels. By contrast, fricatives do not show this kind of regular pattern, and the messier picture they produce on the spectrogram is
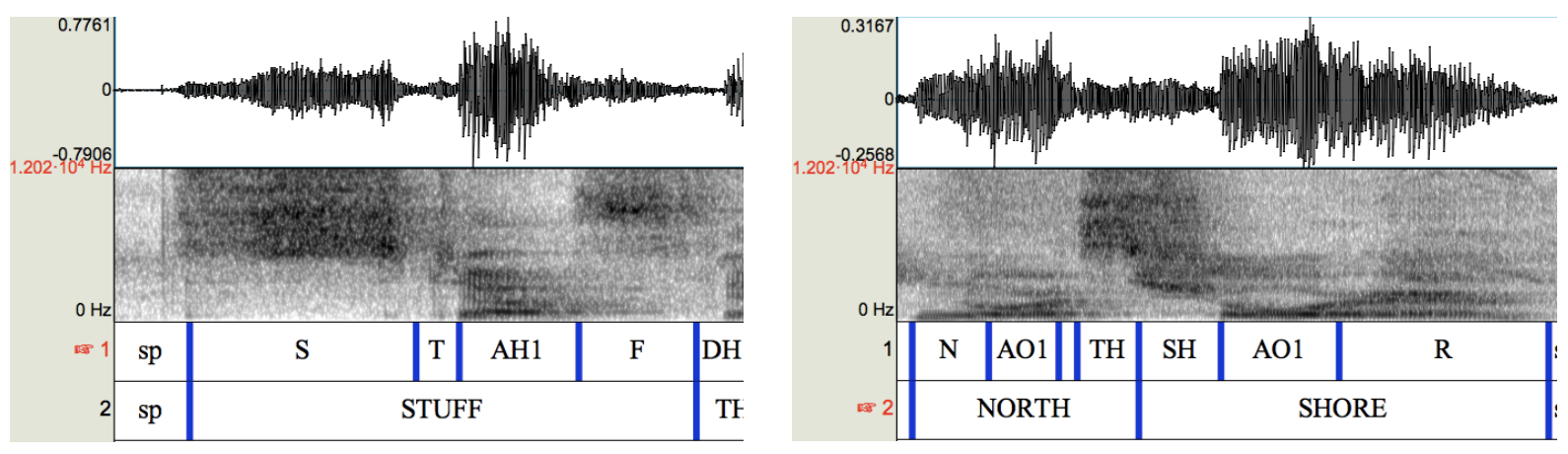

Figure 5.1: Samples of fricatives from the speech of Caroline (25 year-old straight woman): $[\mathrm{s}],[\mathrm{f}],[\theta]$ and $\left[\int\right]$ (from left to right) 

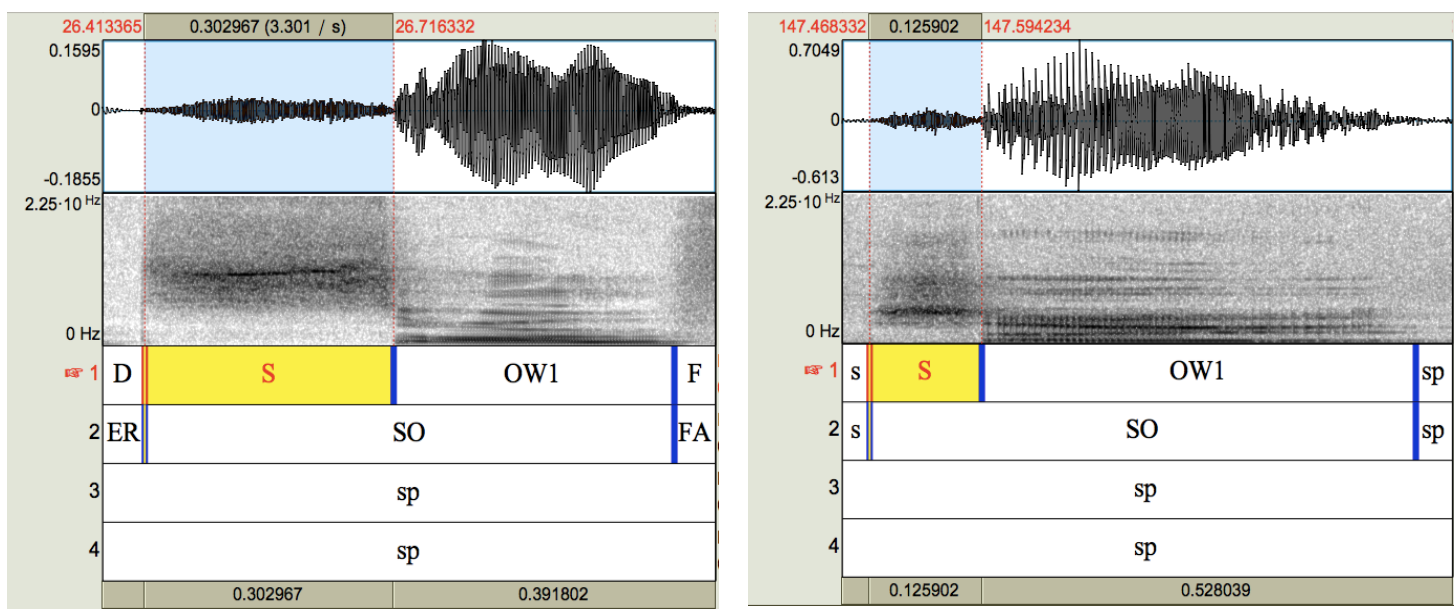

Figure 5.2: The word so spoken by [left] Illeana (23 year old queer woman) and [right] Richard (26 year old trans man).

diagnostic of the manner of articulation. Second, we can see that pattern of energy dispersion is different between the fricatives as well: [f], for example, has a relatively narrow band of high energy (darker band in the spectrogram), while [s] covers a much wider range of frequencies.

Finally, although a spectrogram such as this does not represent a direct measure of peak frequency, we can nevertheless see how these four fricatives have energy concentrated across different bands of frequencies; this is particularly clearly illustrated in north shore, where there is an abrupt change in frequency at the transition point between the $[\theta]$ and the $[\delta]$. Knowing that higher frequencies correspond to fronter articulations, we can quite clearly see that [f] is produced further forward than [s], which in turn is further forward than [J] - which corresponds to what we know about the places of articulation for these sounds. The difference between [s] and [ $\theta]$ is harder to visually identify, which is not surprising given how close they are in place of articulation (see the IPA Chart in Appendix A).

We can also apply this same reasoning to different examples of the same fricative, as in Figure 5.2, which shows the word so produced by two speakers. Again, the vertical axes are the same scale in the two spectrograms, and it clear that the [s] produced by Illeana (a 23 year old queer woman, sampled in the left image) has markedly different spectral characteristics than that produced by Richard (a 26 year old trans man), despite the fact that they are saying the same word (which controls for any effect of phonetic environment). In these two images, because of the narrowness of the range of frequencies across which energy is being maximally expended (i.e., the darkest band in the spectrogram), it is easy to see that these two productions of [s] have different peak frequencies, and therefore likely differ in precise place of articulation. They will also sound different because of these spectral variations, with the higher- 
frequency fronted tokens having a perceptibly higher hissing or whistling quality than the lower-frequency and retracted tokens, which can - in extreme cases - begin to sound more like [J]. These different perceptual correlates have been linked with both gender and sexuality in previous literature (see $§ 5.3$ below for a more detailed discussion), which makes them ideal candidates for a project such as this one, which is principally concerned with the linguistic performance of gendered identity.

This chapter will use descriptive and multivariate analyses to examine a subset of English fricatives known to have a high degree of variability in their production, the sibilants.

\subsubsection{Sibilants}

In English, sibilants are a small subset of the fricatives, and are characterised by a secondary source of turbulent noise. The first is caused by the main constriction between the tongue and the other articulators (depending on where in the oral cavity the fricative is produced), while the second is caused by "a high-velocity jet [...] projected against an obstruction (the teeth, particularly the upper teeth) creating an additional eddying turbulence around the edges of the teeth" (Catford 1968: 322). This gives the English sibilants a particularly noisy, hissy kind of sound that is not found in nonsibilant fricatives. The set of English sibilants is given in (2) below.

[s] as in say, bassoon, and grass

[z] as in $\underline{z}$ oom, bazaar, and $\operatorname{dog} \underline{\mathbf{s}}$

[S] as in ship, condition, and wish

[3] as in leisure, Asian, beige

These four sounds - which, as we can see, can differ in how they are represented in English spelling - differ from each other in systematic ways. [s] and [z] share the same place of articulation, but differ in voicing, as do [S] and [3]. On the other hand, [s] and [S] agree in voicing, but differ in place of articulation, as do [z] and [3] (Table 5.1).

These phonemes also differ distributionally, with [3] showing up only wordmedially in English and -finally, and in fact, only in a small number of words. This is because it is not a phoneme that is native to English, and shows up primarily in borrowed words or in words that are derived from borrowed words (such as, e.g., Malaysian). The other three sounds, as we can see, are free to show up word-initially, word-medially, and word-finally.

\begin{tabular}{rcc}
\hline Table 5.1: & English sibilants \\
\hline & alveolar & post-alveolar \\
voiced & $\mathrm{S}$ & $\int$ \\
voiceless & $\mathrm{z}$ & 3 \\
\hline
\end{tabular}




\subsubsection{What this chapter aims to do}

The goals of this chapter are two-fold. Primarily, we are interested in how much social information can be encoded in the phonetic variation that we observe in sibilant production; to a lesser extent, we are interested in the phonetics of sibilants in New Zealand more broadly. The following sections will detail the methodology and analyses, after a brief tour of previous research in this area.

\subsection{Spectral moments}

The acoustic analysis of fricatives tends to focus on the frequencies across which there is an expenditure of energy. Some researchers (e.g., Stuart-Smith 2007) use peak frequency, which refers to the frequency at which the maximum energy is expended in the spectrum of the fricative. Using the peak frequency (also referred to as spectral peak) allows the researcher to make quite clear inferences about the tongue position during articulation, as there is a direct relationship between tongue position (on a frontback dimension along the palate) and frequency, with higher frequencies being associated with more fronted productions (e.g., Stevens 1998; Jongman, Wayland \& Wong 2000). In particular, peak frequency correlates with the size of the front cavity, "the space in front of the tongue comprising part of the palate, the teeth and the lips" (Levon \& Holmes-Elliott 2013: 112). It should be noted that this relationship holds true only for fricatives with a constriction along the roof of the mouth; labiodental and dental fricatives (such as /f/ and / $/ /$ ) are not produced with enough of a front cavity for this association to be meaningful (Johnson 2003: 124-129; Stevens 1989: 25). Additionally, Jongman et al. (2000: 1254) point out that spectral moments are only reliable indices for distinguishing fricatives that are sibilant - and these are restricted, in English, to /s, z, J, 3, tf, dz/ (e.g., Zsiga 2013: 266). These are all alveolar or postalveolar, and are phonetically similar to one another in many respects. Consequently, any metric that relates to place of articulation will be a useful one in differentiating between these two sounds.

Not all researchers make use - or at least, exclusive use - of peak frequency in the study of fricatives. Although this method does provide useful information about the place of articulation, that is the only articulatory information it can provide, and even that is potentially of limited use. As Johnson (2003: 130) points out, fricatives tend to be characterised by more than one spectral peak, and which of these peaks has the greatest amplitude can vary between utterances, from speaker to speaker and from moment to moment for any given speaker.

The more commonly used approach is to measure the spectral moments of a fricative. Spectral moments are "identical in principle to statistical moments" which "describe a distribution, be it a distribution of scores on a behavioural test or of 

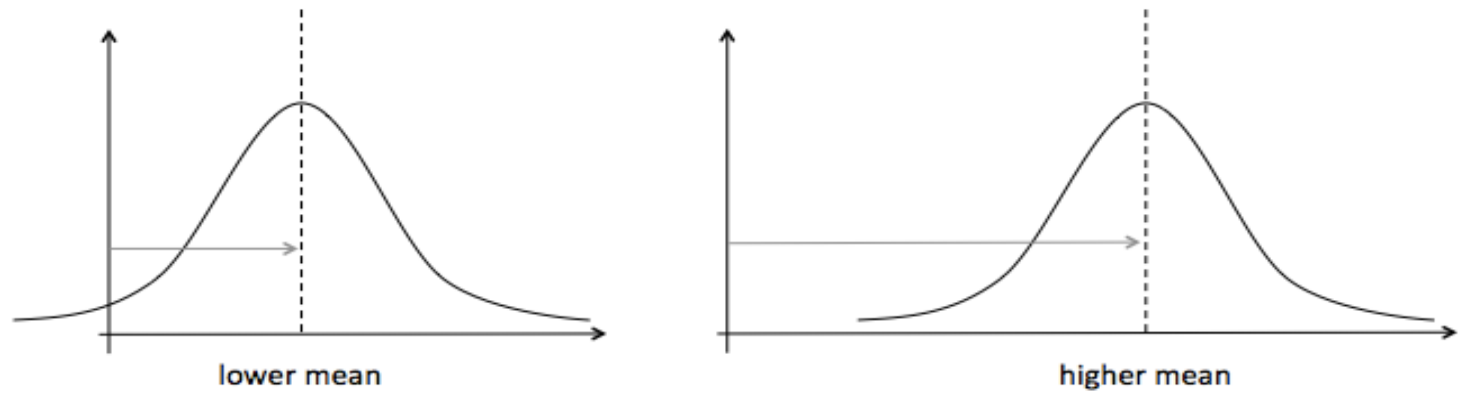

Figure 5.3: First moment: Centre of Gravity (weighted mean)

frequencies in a spectral section" (Nittrouer 1995: 521). They can be understood as indicators of deviation from a normal distribution, in terms of shape and weighting. As "numerical indices of [the] spectral shape and center of gravity" for fricatives (Forrest, Weismer, Milenkovic \& Dougall 1988: 116), these four central moments can provide more detailed information about the acoustics of a given segment. Each is discussed briefly below, and a diagram is provided showing how each relates to a normal distribution.

\subsubsection{First moment: Centre of Gravity}

The first spectral moment is the Centre of Gravity (CofG) (Figure 5.3). It is a weighted mean of the distribution of energy across the spectrum (Thomas 2011: 110; Nittrouer 1995: 521), a "measure of where the centre of the energy concentration produced by the frication lies" (Heffernan 2004: 77), and is measured in Hertz. Like peak frequency, CofG is related to place of articulation for oral fricatives, although it is necessarily less precise as it subsumes a range of frequency peaks across which energy is expended. Nevertheless, speakers with a higher CofG are producing more fronted forms than those with a lower CofG.

\subsubsection{Second moment: Standard Deviation}

Sometimes referred to as 'variance' (although the two terms are not technically interchangeable), Standard Deviation describes the variability of a distribution about its mean (Nittrouer 1995: 521; Heffernan 2004: 77) (Figure 5.4). It, also, can be measured in Hz. Thomas (2011) notes that "fricatives with energy that's relatively concentrated at certain ranges of frequencies, such as [s] and [J], will have lower variance than fricatives with more distributed energy and flatter spectra, such as [ $\theta]$ and [f]" (p.110); as such, Standard Deviation is perhaps more useful in distinguishing fricatives that are less phonetically similar than those that we will focus on here. 

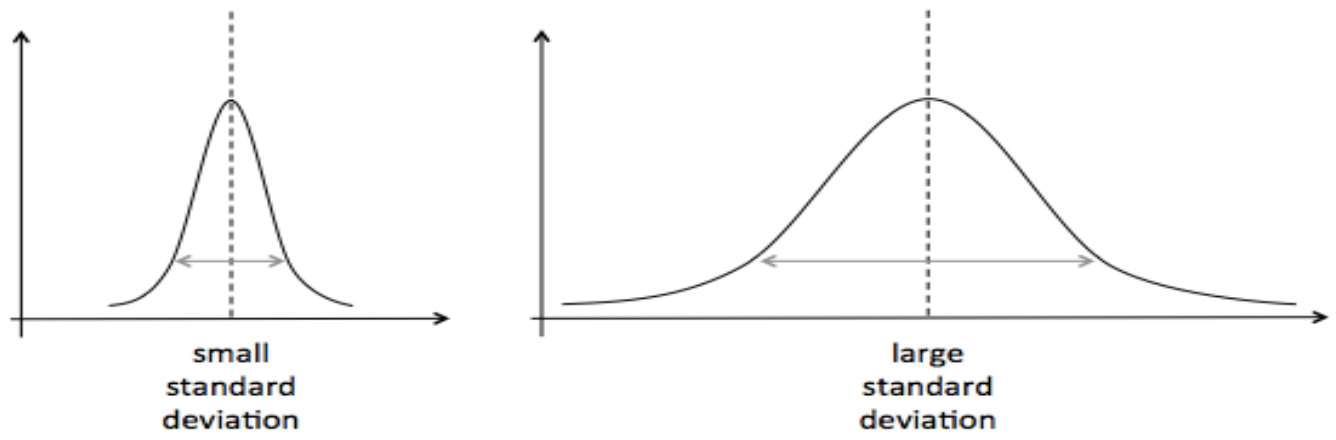

Figure 5.4: Second moment: Standard deviation

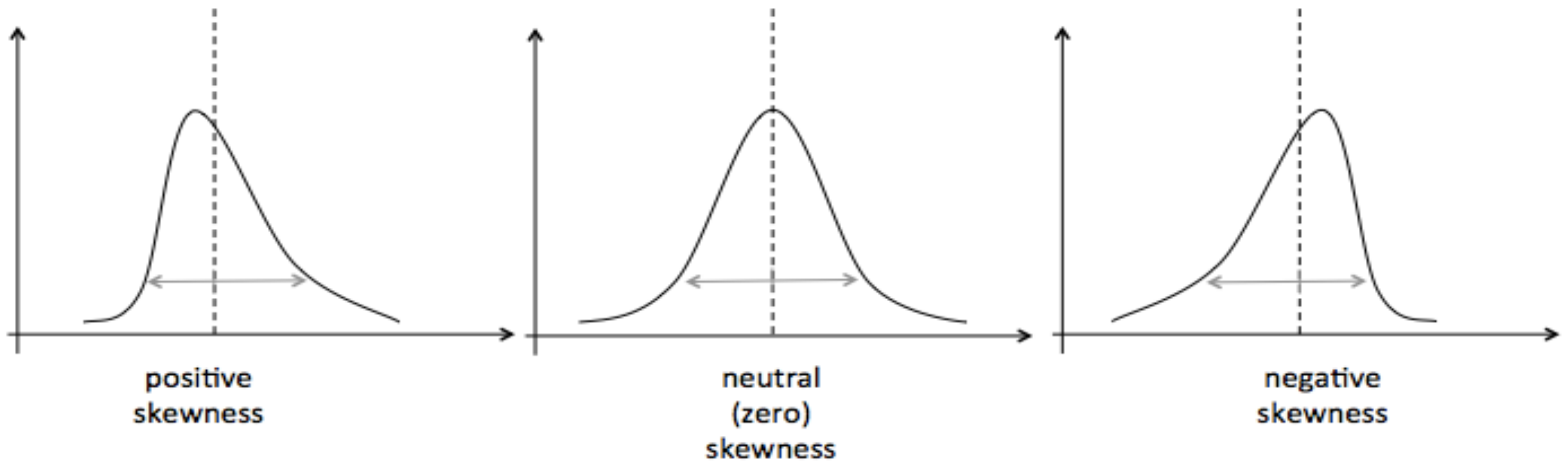

Figure 5.5: Third moment: Skewness
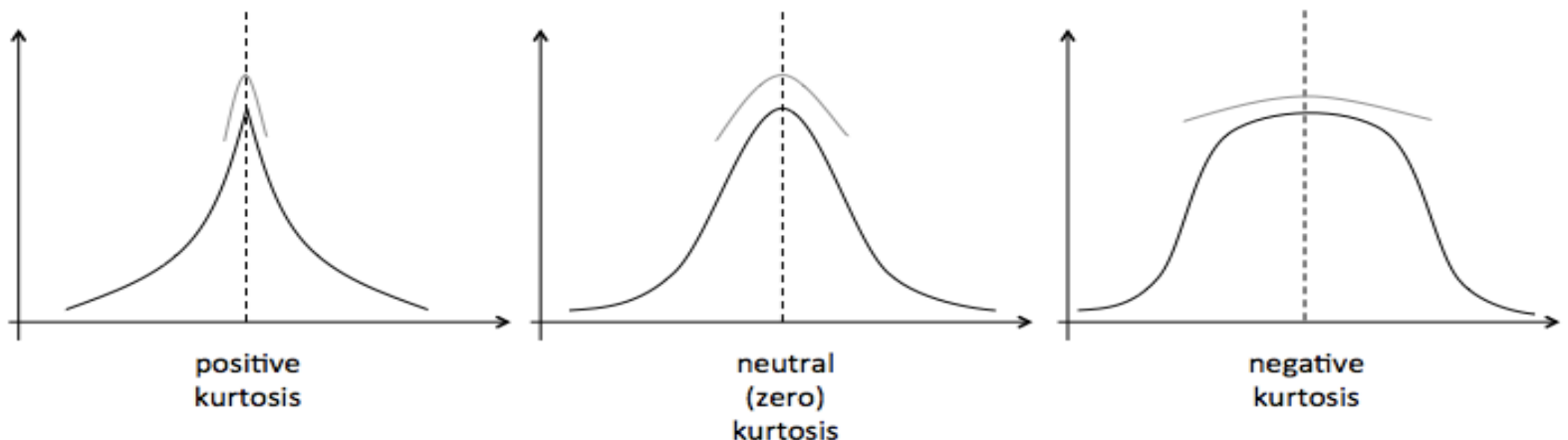

Figure 5.6: Fourth moment: Kurtosis 


\subsubsection{Third moment: Skewness}

Skewness is a "measure of how much the shape of the distribution below the Centre of Gravity differs from the shape above the Centre of Gravity" (Heffernan 2004: 77) (Figure 5.5). This spectral tilt (Thomas 2011: 110) describes how rapidly, and how symmetrically, energy levels fall off as you move away from the mean. It is measured in units of skewness, which represent an entirely mathematical relationship with no clear correlates with the real world. Negative skewness values are associated with distributions that are bunched towards the higher range of frequencies and trail off on the lower end; positive skewness values are associated with distributions that are bunched towards the lower frequencies and trail off on the higher end; and a skewness of zero is associated with a symmetrical distribution (Haley, Seelinger, Mandulak \& Zajac 2010: 551).

\subsubsection{Fourth moment: Kurtosis}

Kurtosis refers to the peakedness of the distribution (Thomas 2011: 110; Nittrouer 1995: 521), and pertains to the shape of the central section of the distribution (Heffernan 2004: 77) (Figure 5.6). A positive kurtosis indicates a relatively pointed shape near the mean, while a negative kurtosis indicates a much flatter shape near the mean (Press, Flannery, Teukolsky \& Vetterling 1988: 612). Jongman et al. (2000) note that $/ \mathrm{s} /$ and $/ \mathrm{z} /$ tend to have greater kurtosis in general than the other English fricatives.

\subsubsection{Fricative moments and gender}

The study of fricatives - and sibilants in particular - as a marker of gender and sexuality is not a novel endeavour, so there are established practices in place. We will focus on CofG (Pharao, Maegaard, Møller \& Kristiansen 2014; Tabain 2001; Newman, Clouse \& Burnham 2001; Flipsen, Shriberg, Weismer, Karlsson \& McSweeny 1999; Zimman 2012) and skewness (Pharao, Maegaard, Møller \& Kristiansen 2014; StuartSmith 2007; Flipsen et al. 1999; Munson, McDonald, DeBoe \& White 2006), as they have reliably been found to correlate with gendered aspects of identity in previous studies of sociophonetic variation in English.

\subsubsection{What are Centre of Gravity and Skewness actually measuring?}

What do these spectral moments actually correlate with in the real world, either acoustically and/or articulatorily? This is actually a complicated question to answer, as the methods needed to record the fine articulatory details (such as an electropalatographic, or EPG, plate) necessarily interfere with the naturalistic production of alveolo-palatal fricatives, and so can affect the acoustic output.

One study that did attempt to explore the relationship between articulatory gestures and acoustic measurements was Fuchs \& Toda (2010), who were able to make a few general observations (summarised in Figure 5.7). On the articulatory side of the 


$$
\left(\begin{array}{l}
\text { fronter articulation } \\
\text { narrower gap }
\end{array}\right) \Rightarrow\left(\begin{array}{l}
\text { higher frequency } \\
\text { lower skewness }
\end{array}\right)
$$

Figure 5.7: Articulatory and phonetic correlates of $/ \mathrm{s} /$ (based on Fuchs \& Toda 2010)

question, they noted that female speakers of both English and German generally produced a more fronted /s/ than males, as well as a narrower constriction (the opening through which the air passes). Acoustically, they found that females tended to have higher centre of gravity (moment 1 ) and lower skewness (moment 3 ) than males.

They were able to statistically relate these articulatory dimensions and acoustic parameters, but only at a relatively broad level - they did not establish a one-to-one mapping of a particular dimension to a specific measure. It is not at all clear, in fact, that such a linear relationship is even possible, or whether the possibility can even be assessed with anything less comprehensive than an experimental fMRI study, given the range of articulatory gestures that are likely to contribute to the overall acoustic effect, and the high likelihood of coarticulation.

It is also worth noting that, although Fuchs \& Toda did not discuss this in their 2010 study, the EPG frequency plots they published show a tendency for men to have a larger area of contact between tongue and palate - as well as having a dimension of width to the constriction, there is also a dimension of length. If one visualises the constriction as a tube formed by the tongue and the palate, this tube can be relatively short (where the constriction is restricted to a small portion of the back-front axis of the palate) or long (where there is a greater area of tongue contact against the palate). This may be an inevitable consequence of having a more retracted place of articulation (given the shape of the roof of the mouth), or it may constitute a separate articulatory action; in either event, this may be another mechanism influencing the acoustics of sibilants.

\subsection{Why sibilants?}

There are a number of persistent stereotypes about the speech style of gay men, including that of the 'gay lisp'. The feature being alluded to is not really a lisp in a technical sense, which in the field of speech-language pathology and therapy refers to a difficulty in articulating /s/ and / $\mathrm{z} /$ sounds that most commonly results in the sounds being produced as interdental fricatives (e.g., Bowen 2011), and can often lead to communicative difficulty. It is certainly not the case that gay men are producing anything quite as fronted as $/ \theta /$ or $/ ð /$ when they are aiming for $/ \mathrm{s} /$ or $/ \mathrm{z} /$, but to the nonlinguistically inclined, any articulation that is fronter than expected may get classified as 
'lisping'. It is perhaps more accurate, then, to talk about the phenomenon as part of what Cameron and Kulick (2003: 96) call "the voice", a set of articulatory and phonetic features that have come to index gay male identities in many English-speaking, Western cultures.

\subsubsection{Sibilants, sex, and gender}

Two early reports on the phonetics of fricatives arrived independently at the conclusion that listeners are able to identify speaker sex based solely on voiceless fricatives heard in isolation. That is to say, listeners have significantly better-thanchance performance in telling if a speaker is male or female, even when there is no fundamental frequency (voice pitch) information available. Ingemann (1968) reported that $/ \mathrm{h} /, / \mathrm{s} /$, and $/ \mathrm{s} /$ provided particularly reliable evidence for listeners, while Schwartz (1968) found that $/ \mathrm{s} /$ and $/ \mathrm{J} /$ were accurate cues but $/ \mathrm{f} /$ and $/ \theta /$ were not.

This difference was ascribed to females having fricative spectra "not only $[\ldots]$ higher in frequency than the male but parallel to them as well" (Schwartz 1968: 1179), and later studies confirmed this. Jongman, Wayland \& Wong (2000) report a mean spectral peak for [s] for females around $7.5 \mathrm{kHz}$, while that of males is around $6.1 \mathrm{kHz}$. In a meta-analysis of 21 studies that vary widely in methodologies and analysis, Flipsen et al. (1999) report that males are found to have a peak frequency for [s] in the range of 4 to $7 \mathrm{kHz}$, while that of females is in the range of 6.5 to $8.1 \mathrm{kHz}$.

Some researchers (e.g., Daniloff, Wilcox \& Stephens 1980; Stevens 1998) have attributed these observed differences to the physiological characteristics of adult males and females. Females, being physically smaller overall, have a smaller vocal tract and therefore a smaller front cavity (the region between the constriction made with the tongue, and the lips), which results in higher frequencies for fricatives.

While this explanation may seem intuitively appealing, it does not stand up to close scrutiny. Beck (2010) finds that speaker sex is equally recoverable for preadolescent speakers, where there are essentially no anatomical differences between male and female vocal tracts. Strand (1999) observes that the principal differences between the vocal tracts of males' and females' oral tracts are in the back cavity (behind the articulatory constriction), which is not a prominent component of the spectral properties of fricatives (e.g., Shadle 1985, 1991; Ladefoged \& Maddieson 1996). Strand proposes that the difference between males and females is better explained by males producing a more retracted variant of [s].

In a study of fricatives in Glasgow, Stuart-Smith (2007) found that the spectral differences in fricatives between males and females varied depending on the social factor of class, with younger working class women producing a much more masculinesounding [s] than their older or middle class counterparts. As there is no evidence for anatomical vocal tract differences between working and middle class women of the same age, she argued that these differences were "part of the construction of socially- 
distinct gendered identities" (p.75). Interestingly, she found no comparable pattern for $/ \mathrm{J} /$, and concluded that in Glasgow, "the phonological contrast between /s/ and / $/$ [seems to be] complemented by a socio-indexical contrast such that $/ \mathrm{s} /$ is used to index specific social identities, but $/ \mathrm{g} /$ is not" (p.82).

Other studies have also noted that observed gender differences in fricative spectra are dependent on non-physiological factors. Heffernan (2004) found robust differences between men and women for the spectral properties of /s/, as well as differences between speakers of Canadian English and those of Japanese. Fuchs \& Toda (2010) compared speakers of German with speakers of English, and found differences considerably more pronounced between English-speaking men and women than between their German-speaking counterparts. Levon \& Holmes-Elliott (2013) looked at /s/ in two British reality TV shows, and found gender differences were more pronounced for speakers from a working-class neighbourhood than those from an upper-class one. Within the New Zealand context, Starks (2000) found that Pacific Island and NZ European women had a fronted /s/ more often than the corresponding men, but that this difference was absent with Maori people, whose rates of use were comparable with the men in the other groups.

With the spectral characteristics of at least some (if not all) fricatives tied to social categories of gender rather than to biological categories of sex, then fricatives are potentially a site for quite nuanced gendered identity work.

\subsection{2 [s] and sexuality}

With stereotypes of gay-sounding men sounding 'lispy' and effeminate, the acoustic correlates of different kinds of masculinity became an object of study in phonetics. Pitch properties and stop release were often explored, as were fricatives. Avery and Liss (1996) found differences between more-masculine-sounding and lessmasculine-sounding men on three of the four spectral moments for both $/ \mathrm{s} /$ and $/ \mathrm{J} /$, and Crist (1997) reported a lengthening of /s/-frication in onset consonant clusters when speakers were performing "the queeniest, most flamingly gay stereotype" (p.54) that his participants could produce. Linville (1998) found listeners were able to correctly identify the sexual orientation of speakers from short sound clips around $80 \%$ of the time, and noted that the speakers identified as gay had higher peak frequencies and longer durations for /s/ than those identified as straight; Smyth and Rogers (2008) reported similar patterns with gay-sounding men in Toronto. In a study of gay men's speech in Wellington, New Zealand, Taylor (1998) found gay men that used a gaysounding /s/ (described as having a sharp spectral peak) more than twice as often as straight men (who tended to produce an $/ \mathrm{s} /$ with a flatter, more diffuse spectrum). He found no correlations with age, socioeconomic status or degree of integration with the gay community; in his data, /s/ "appears not to participate in any obvious pattern aside from a gay/straight distinction” (p.127). 
The gendered and gendering properties of /s/ are not restricted to English. Maegaard \& Pharao (2016) conducted a study of /s/ and masculinity in Copenhagen, Denmark, and found two distinct patterns. In modern Copenhagen Danish, a fronted /s/ produced by men is associated with homosexuality and femininity; by contrast, in street Copenhagen Danish, this same articulation is associated with toughness and immigrant masculinity. Clearly, then, $/ \mathrm{s} / \mathrm{can}$ index a range of masculinities, depending on context.

And what of femininities? If a particular variant of $/ \mathrm{s} /$ in men is associated with femininity and sounding gay, is there a corresponding association in the speech of women? This question has been much less exhaustively studied than it has been for men, in part no doubt because the speech of lesbians is far less commented-on in general than that of gay men. Whether this is because there are genuinely fewer salient differences between women, or because the phonetic behaviours of different femininities is less strictly policed, is an open question (although the linguistic behaviours of women generally are policed). Nevertheless, some researchers have explored the area. Munson, Jefferson and McDonald (2006) investigated the relationship between fricatives and the perception of sexuality in women, and found that women rated as sounding lesbian or bisexual had lower CofG for $/ \mathrm{s} /$ than straightsounding women. I have previously (Hazenberg 2012) found non-significant differences between straight and queer women, with straight women having slightly higher CofGs. Podesva \& Van Hofwegen (2016) also found lesbians producing/s/ with a lower CofG than straight women, in a small, rural, and conservative town in California. They also noted that, although the gay men in their study had higher CofG than their straight counterparts, they were actually considerably lower than those found in most previous studies - an effect that they attributed to locally-constructed identities, nothing that "variation patterns are constrained by the community's sociopolitical landscape" (p.186).

So /s/ at least - and potentially other fricatives, such as $/ \mathrm{S} /$ - can be used to index a range of femininities and masculinities, with varying degrees of salience to the speech community in general. Given the gendering power of this variable, then, how is it deployed by transsexual and transgender people?

\subsection{3 [s] and trans identities}

Much of the phonetic research conducted on transsexual voices is directly linked to therapeutic contexts, aimed at helping (mainly) trans women develop a voice that they are happy with. The field of transsexual and transgender phonetics as a window into gendered linguistic practice, as opposed to clinical purposes, is a relatively recent one, but the fricatives $/ \mathrm{s} /$ and $/ \mathrm{g} /$ have featured prominently in the existing literature.

Looking at the relationship between sexual orientation and disclosure/visibility among trans men in the US, Papp (2011) found some complex patterns with the spectral 
moments of $/ \mathrm{s} /$ and $/ \mathrm{J} /$. Trans men attracted to men had the highest CofG among her groups of speakers for both fricatives, and the lowest skew for $/ \mathrm{s} /$. She also noted that trans men who were 'stealth' - who lived as men but did not disclose their transsexuality to non-intimate acquaintances - had more positive skew in $/ \mathrm{s} /$ than those who were not, a pattern which other researchers (e.g., Jongman et al. 2000; Zimman 2013) associate with feminine- and gay-sounding speech. Duration was also a significant factor, with straight-identified trans men (those attracted exclusively to women) producing the shortest fricatives.

Zimman's $(2012,2013)$ work on the speech of trans men found that those who identified as heterosexual had lower CofGs for $/ \mathrm{s} /$ than those who identified as queer men or as existing outside of the gender binary (e.g., genderfluid, genderqueer, etc.). He noted that although this accompanied a general lowering of F0 (fundamental frequency), the two were not directly correlated, meaning that the manipulation of /s/ was used as a way to perform gender identity that was separate from the physiological changes induced by hormone therapy.

Working with Canadian English (Hazenberg 2012), I looked at/s/ across younger straight, queer, cissexual and transsexual people in Ottawa, Ontario, and noted that women (including trans women) had universally higher CofG than men (including trans men), and universally lower skew. Trans women, queer (cis) men and trans men were clustered towards the middle of the pack, while straight men and women occupied the extremes of both scales. Straight men had generally smaller deviations from the mean than anyone else, suggesting that they were socially more constrained in the range of $/ \mathrm{s} /$ articulations that was available to them.

Podesva and Van Hofwegen (2016) found that trans men in rural California had higher CofGs than those in San Francisco (Zimman 2013), and trans women had lower CofGs than those in Ottawa (Hazenberg 2012).

\subsubsection{A quick note on voicing}

Research on [s], gender, and sexuality has focused on voiceless fricatives, partly because that is where previous studies have reported differences, and also possibly because of the confounding effect of voicing. It is well-known that men tend to have a lower fundamental (F0) frequency than women (e.g., Peterson \& Barney 1952), so there is always the possibility that differences in voicing will distort the patterns that are clearly visible in voiceless segments. In fact, Hayward (2000: 192) notes that voiced fricatives "have a low-frequency voice bar across the bottom of the spectrogram", but that "[a]bove the voice bar, the overall pattern will be similar to that for the corresponding voiceless fricatives, but the intensity of the friction will be weaker". So in principle, at least, there is no reason not to examine gendered differences in voiced sibilants, particularly if we limit our analysis to frequencies above F0. 


\subsubsection{Summary of previous findings}

Generally, research has found that women produce fricatives with higher peak frequencies or centres of gravity than men, and that $/ \mathrm{s} /$ and (to a lesser extent) $/ \mathrm{J} /$ are particularly salient gender-indexing variables. Phonetic differences between men and women are mainly due to social factors rather than to physiological differences, as evidenced by class affiliations and sexual orientation effects. /s/ in particular seems salient enough that queer and trans speakers are able to manipulate their articulation to more accurately perform their gendered identities.

Of the studies that have investigated the phonetics of $/ \mathrm{s} /$, many have reported either Centre of Gravity (CofG) or spectral peak/peak frequency for their data. Table 5.2 provides a summary of some of these by broad general category. Where a study subdivided one of these categories in one way or another, their results are presented here as a range. In some cases, the frequencies were not directly reported numerically

\begin{tabular}{|c|c|c|c|c|c|c|}
\hline \multicolumn{7}{|l|}{ /s/ CofG across studies (in kHz) } \\
\hline \multirow{2}{*}{ 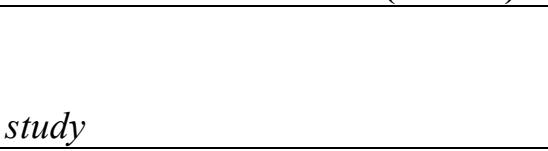 } & \multicolumn{2}{|c|}{$\begin{array}{l}\text { straight or } \\
\text { unspecified }\end{array}$} & \multicolumn{2}{|c|}{ queer/LGBT } & \multicolumn{2}{|c|}{ trans } \\
\hline & women & men & women & men & women & men \\
\hline Podesva \& Van Hofwegen 2013 & $7.15-7.73$ & 5.25 & 6.32 & 6.16 & 6.05 & 6.53 \\
\hline Zimman 2013 & & $5.61-7.18$ & & $5.87-7.23$ & & $5.58-6.66$ \\
\hline Papp 2011 & & & & & & $7.46-8.85$ \\
\hline Fuchs \& Toda 2010 (English) & 6.41 & 5.63 & & & & \\
\hline Heffernan 2004 (English) & 8.28 & 7.49 & & & & \\
\hline Munson, Jefferson \& McDonald 2006 & 7.70 & 6.50 & & & & \\
\hline Tabain 2001 & $6.58-7.66$ & & & & & \\
\hline Zimman 2012 & & & & & & $5.73-8.96$ \\
\hline Hazenberg 2012 & 7.50 & 4.49 & 7.23 & 6.29 & 6.61 & 6.14 \\
\hline Nittrouer 1995 & 8.00 & 6.10 & & & & \\
\hline Fuchs \& Toda 2010 (German) & 5.86 & 5.46 & & & & \\
\hline Heffernan 2004 (Japanese) & 8.41 & 8.00 & & & & \\
\hline \multicolumn{7}{|c|}{ /s/ spectral peak (peak frequency) across studies (in $\mathrm{kHz}$ ) } \\
\hline \multicolumn{7}{|c|}{$\begin{array}{ll}\text { straight or } \\
\text { unspecified }\end{array}$} \\
\hline study & women & men & & & & \\
\hline Schwartz 1968 & 6.50 & 5.50 & & & & \\
\hline Jongman, Wayland \& Wong 2000 & 7.50 & 6.20 & & & & \\
\hline Fuchs \& Toda 2010 (English) & 8.28 & 6.28 & & & & \\
\hline Levon \& Holmes-Elliott 2013 & $7.00-8.10$ & 4.90 & & & & \\
\hline Stuart-Smith 2007 & $5.54-7.91$ & $4.80-5.29$ & & & & \\
\hline Fuchs \& Toda 2010 (German) & 5.84 & 5.72 & & & & \\
\hline
\end{tabular}


in the publication, so the figures below are estimates read from charts and diagrams. A more comprehensive comparison of studies - but one which does not focus on the range of gendered identities targeted in this study - is provided in Flipsen et al. (1999).

In English generally, there is a gendered difference in the frequency of /s/ regardless of the measure used, with women producing higher frequencies. Interestingly, these differences are not present in the data reported on German or Japanese, meaning that the social information conveyed by $/ \mathrm{s} /$ is not a universal association, but one constrained by social factors particular to the language and culture of the speakers.

\subsection{Methods}

Because /s/ has been so frequently studied, there exist fairly well-established protocols for the phonetic analysis of fricative spectral moments. For examining gender and $/ \mathrm{s} /$, Flipsen et al. (1999) recommend measuring at the midpoint of the segment, using a linear scale, controlling for the phonetic context, and focusing on the first and third spectral moments. In this study, we take measurements in Hertz over the central $40 \mathrm{~ms}$ of the fricative, and exclude all tokens where the following or preceding segment is either a fricative or an affricate. Measurements for all spectral moments were taken, as Flipsen et al.'s recommendations were tailored to the study of $/ \mathrm{s} /$, and we are considering more than one fricative in this study. It transpired, however, that statistically-significant differences along gendered identity lines occurred almost exclusively with the first and third moments, so the focus of the following discussion will be, as Flipsen et al. recommended, focused on these two moments.

As discussed in $\$ 3.5 .2$, a script in Praat (Appendix B) was used to automatically measure the spectral properties of all tokens of $/ \mathrm{s} /, / \mathrm{z} /$, and $/ \mathrm{J} /$ in the transcribed portions of the ATC. This output included detailed information about the phonetic environment and lexical context for each instance, which allowed the exclusion of certain tokens. In particular, the following were excluded:

- sibilants under $40 \mathrm{~ms}$ in duration: extremely short tokens are unlikely to be fully articulated, and any measurements taken will likely not include a stable central portion that is representative of the segment in that particular phonetic context

- sibilants adjacent to other fricatives, whether they identical segments (e.g., cross

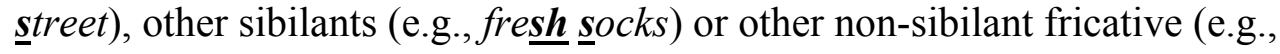
cross fit): this is to minimise the effect of alignment error on the part of the forced aligner (see \$3.5.1), which could potentially place segment boundaries inaccurately between fricatives, which in turn would result in inaccurate spectral measurements

- $\quad$ sibilants adjacent to affricates (e.g., church sermon, less $\underline{\text { ch }}$ ance): although in 
principle the stop portion of the affricate should make the preceding fricative distinct from the following segment in less chance, articulatory shortcuts in spontaneous speech may result in a lenited affricate, which would give rise to the same problem as that noted above about inaccurately-placed segment boundaries - it was simpler to bulk-exclude any such potentially confounding collocations

- sibilants in incomplete words where the phonetic environment could not be recovered (e.g., $I \underline{s}--)$ : the analysis that follows includes the following and preceding segments, so to ensure comparable data, tokens where this information was not present were excluded; note that audible pauses were retained

\subsubsection{Correcting for biological differences?}

As with anything speech-related, any comparison between women and men raises the question of normalising to correct for biological differences between the sexes. This is particularly true for variables such as vowels, which rely on oral and nasal resonances to amplify key harmonics of the fundamental frequency (F0). It is known that women have significantly smaller oral and nasal cavities than men, and that women also have higher F0s than men (e.g., Fant 1960; Whiteside 1996), so these are two key parameters that must be normalised before any comparison can be made.

With fricatives, however, it is not standard practice to normalise for biological differences. Partly this is because the acoustic properties of fricatives do not rely on F0 or on the overall dimensions of the vocal tract, and partly because previous research (see $\$ 5.3$ above) has shown that differences are primarily attributable to social rather than biological factors. Fuchs \& Toda's (2010) carefully controlled study concluded that "[s]ignificant results for morphological parameters were not found that correlated with the sex of the speaker" (p.298). Following in the traditions of previous researchers, then, the data in this study of sibilants is not normalised to correct for biological factors.

\subsubsection{Tokens}

Initially, the spectral moments for four fricatives were extracted: /s/, $/ \mathrm{z} /, / \mathrm{J} /$ and

/3/. However, the occurrences of $/ 3 /$ were so infrequent that it was not possible to collect enough tokens to do any kind of analysis, or even discussion, whatsoever, so this segment was dropped from the investigation. Rather than being able to do a systematic comparison between voiced and voiceless pairs of alveolar and palatal fricatives as was originally intended, we will instead compare $/ \mathrm{s} /$ and $/ \mathrm{J} /$ (which differ in place of articulation), and /s/ and /z/ (which differ in voicing). Table 5.3 summarises the number of tokens available for analysis. 


\begin{tabular}{|c|c|c|c|c|}
\hline gender & $/ \mathrm{s} /$ & $\mid z /$ & $\mid \mathrm{g} /$ & $|3|$ \\
\hline oqm & 1375 & 704 & 180 & 8 \\
\hline oqw & 1007 & 522 & 161 & 9 \\
\hline osm & 1580 & 852 & 191 & 16 \\
\hline osw & 1437 & 831 & 294 & 11 \\
\hline otw & 899 & 571 & 177 & 5 \\
\hline yqm & 1512 & 866 & 209 & 17 \\
\hline yqw & 1036 & 531 & 164 & 4 \\
\hline ysm & 1384 & 766 & 179 & 16 \\
\hline ysw & 1548 & 887 & 203 & 5 \\
\hline ytm & 1610 & 904 & 200 & 6 \\
\hline $\mathrm{ytw}$ & 819 & 382 & 111 & 9 \\
\hline total & 14207 & 7816 & 2069 & 106 \\
\hline
\end{tabular}

\subsubsection{1 /s/ and $/ \mathbf{f} /$}

Previous research failed to find gendered patterns for $/ \delta /$ that were comparable in magnitude to those found for $/ \mathrm{s} /$, but that does not mean that this contrast does not bear examining here. Since a fronted $/ \mathrm{s} /$ is generally associated with particular gendered presentations (often women and gay-sounding men) and the difference between $/ \mathrm{s} /$ and $/ \mathrm{J} /$ is essentially one of fronting (where /s/ has a place of articulation slightly ahead of $/ \mathrm{J} /$ ), it is possible that there is a relationship between the articulatory position for $/ \mathrm{s} /$ and $/ \mathrm{d} / \mathrm{s}$

It should be noted that all tokens of $/ \mathrm{st}$ / / were excluded from the data set of $/ \mathrm{s} /$ tokens, as New Zealand English seems to be undergoing a change that retracts $/ \mathrm{s} /$ in this environment (e.g., Lawrence 2000; Hay, Maclagan \& Gordon 2008; for a discussion of this same process in American varieties of English, see Labov 1984; Janda \& Joseph 2003; Durian 2007; Gylfadottir 2015; and for British varieties, Bass 2009; Glain 2014). Because this process is subject to overt commentary in some varieties of English, particularly when produced by young women, the (str) variable was initially included as part of this study. However, it failed to show any strongly gendered effect in this data: there were slightly more tokens that sounded $/ \mathrm{J} /$-like produced by men than by women, but a closer examination showed that the effect seemed to not be produced by retraction alone, but was rather influenced by other co-articulatory effects (e.g., Shapiro 1995; Lawrence 2000). As the effect was slight and the articulatory process more complicated than could be assessed by CofG or Skewness alone, (str) was subsequently omitted from this study.

\subsubsection{2 /s/ and /z/}

Researchers have also failed to find a strong gendered effect for $/ z /$ akin to that found for $/ \mathrm{s} /$, but word-final $/ \mathrm{z} /$ devoicing has been noted in at least some varieties of New 
Zealand English (e.g., Holmes 1996), where it is preferred by speakers of Maori English and by younger, working class women. This devoicing in theory may mean that some of the gendered constraints operating on /s/ may also be relevant to /z/ in New Zealand English.

\subsubsection{Analytical approach}

The results for each of the three fricative $(/ \mathrm{s} /, / \mathrm{g} /, / \mathrm{z} /)$ are presented below. For each variable, two lines of evidence will be considered: a distributional one that includes an ANOVA and subsequent post-hoc test, and a regression model that examines the relative weight of different factor groups. The first analysis was carried out in SPSS (IBM Corp 2013). Following an initial ANOVA to determine whether there were any significant differences overall, a Tukey post-hoc test for homogeneous subsets was carried out, to determine which of our gendered groups differed significantly from which other groups.

For the multivariate analysis, modelling was done using Rbrul (Johnson 2009), a mixed-effects variable rule analysis program run through R (R Core Team 2013) (see $\S 3.6 .2$ ). The social factors tested were gender (masculine, feminine), age (older, younger), identity (straight, queer, trans), and two- and three-way combinations of these factors. Linguistic factors were also included in the analysis (in particular, phonetic environment and position in the word), in order to disentangle any social effects from more structural ones, but these factors are not discussed here. As the transcriptions were time-aligned automatically, duration measurements were found to be unreliable, and so were not included in any of the statistical models.

A number of different statistical runs were conducted for each measurement, varying the combinations of both social and linguistic factors. For example, models were tested that maintained the eleven-way gendered identity category, as well as models that broke the three axes apart (age, gender, and identity) and treated them separately, with the possibility of interactions between them. The best models determined by log-likelihood test - for each measure of each fricative are the ones discussed below.

\subsubsection{A note about independence}

Under this type of analysis, factor groups are expected to be independent of each other, and when they appear not to be, they should be examined for interaction effects. With the data in this project, this is generally true: a speaker's gendered identity is independent of the phoneme preceding an /s/, for example. However, if two groups are completely independent, then we would expect that every combination of factors from within those groups would be equally possible. With language data, that is not strictly true, as some sequences of sounds are much more likely than others. Thus the factor groups of preceding segment and following segment - the sounds immediately 
before and after the fricative in question - are not combinatorially independent of each other. While a sequence of $/ \mathrm{tsb} /$ is possible if we allow for sequence-internal boundaries (e.g., let's be nice [letsbinees]), it exists only in very limited contexts.

In the laboratory setting common to much phonetic work, such limited-context examples can be balanced in the data, so that all contexts can be compared equally. In naturally-occurring speech, however, these types of limited-context phonetic environments are very infrequent, particularly compared to, for example, intervocalic contexts. So if we made a cross-tabulation table of all preceding and all following environments (each comprising between 20 and 30 sound segments), there would necessarily be a high number of unattested combinations. This is an unavoidable consequence of working with natural speech data, and although it limits the generalisations we are able to draw about phonological influences, for example, it does not fundamentally invalidate the methodology, since we can group sets of segments into larger categories (for example, by place or manner of articulation) in our analysis.

\section{$5.5 / \mathrm{s} /$}

Given that $/ \mathrm{s} /$ is the fricative most widely examined in the context of gender and sexuality, it seems like a good place to begin our discussion as well. We will first consider the distributional patterns in both Centre of Gravity (CofG) and Skewness, before turning to the multivariate analysis that attempts to model the effect of social factors while taking into consideration other linguistic factors that may be adding to the complexity of the distribution. This section will conclude with a discussion of $/ \mathrm{s} / \mathrm{as}$ a site for gendered identity performance.

\subsection{1 /s/: Distribution}

Centre of Gravity and Skewness are closely related: distributions with a high peak frequency (and so also a high CofG) tend to have a lower Skewness, and vice versa (Thomas 2011: 110). However, they are not simply two measures of the same property, but rather two related measures; to some extent, they are independently manipulable (Hazenberg 2012: 58), so it makes sense to consider them in tandem.

The distributions will be described in three ways, each providing its own perspective on the data. First we will look at the means and the standard deviations to get a broad sense of the distribution. Next, we will look at the homogeneous subsets derived from post-hoc Tukey HSD tests following an ANOVA, to explore the degree of similarity and difference across gendered groups. Finally, a scatterplot of Centre of Gravity plotted against Skewness is presented, to gauge the relationship between the two measurements, and to visualise any broad gendered trends in the data. 


\begin{tabular}{|c|c|c|c|}
\hline \multicolumn{4}{|c|}{$\begin{array}{l}\text { Table 5.4: } / \mathrm{s} / \mathrm{CofG} \text { - Means and Standard } \\
\text { Deviations }(\mathrm{Hz})\end{array}$} \\
\hline Gender & $N$ & Mean & St.Dev. \\
\hline osm & 1505 & 5365 & 823 \\
\hline otw & 869 & 5370 & 1073 \\
\hline ysm & 1349 & 5661 & 1297 \\
\hline oqm & 1334 & 5864 & 1167 \\
\hline ytm & 1567 & 6424 & 1166 \\
\hline yqm & 1492 & 6610 & 1368 \\
\hline oqw & 969 & 6801 & 1141 \\
\hline osw & 1365 & 7178 & 1154 \\
\hline ysw & 1481 & 7425 & 1272 \\
\hline ytw & 805 & 7526 & 1411 \\
\hline yqw & 1014 & 7553 & 1279 \\
\hline
\end{tabular}

Table 5.5: /s/ Skewness - Means and Standard Deviations (units of Skew)

\begin{tabular}{cccc}
\hline Gender & $N$ & Mean & St.Dev. \\
\hline osm & 1505 & 1.80915 & 1.35754 \\
ytm & 1567 & 0.74038 & 0.82378 \\
otw & 869 & 0.66289 & 0.98791 \\
oqm & 1334 & 0.60492 & 0.75727 \\
yqm & 1491 & 0.44148 & 0.74054 \\
ysm & 1349 & 0.39682 & 0.82119 \\
oqw & 969 & 0.21927 & 1.04542 \\
osw & 1365 & 0.2159 & 1.0374 \\
ytw & 805 & -0.0422 & 0.85043 \\
ysw & 1481 & -0.07259 & 0.86129 \\
yqw & 1014 & -0.45738 & 0.90496 \\
\hline
\end{tabular}




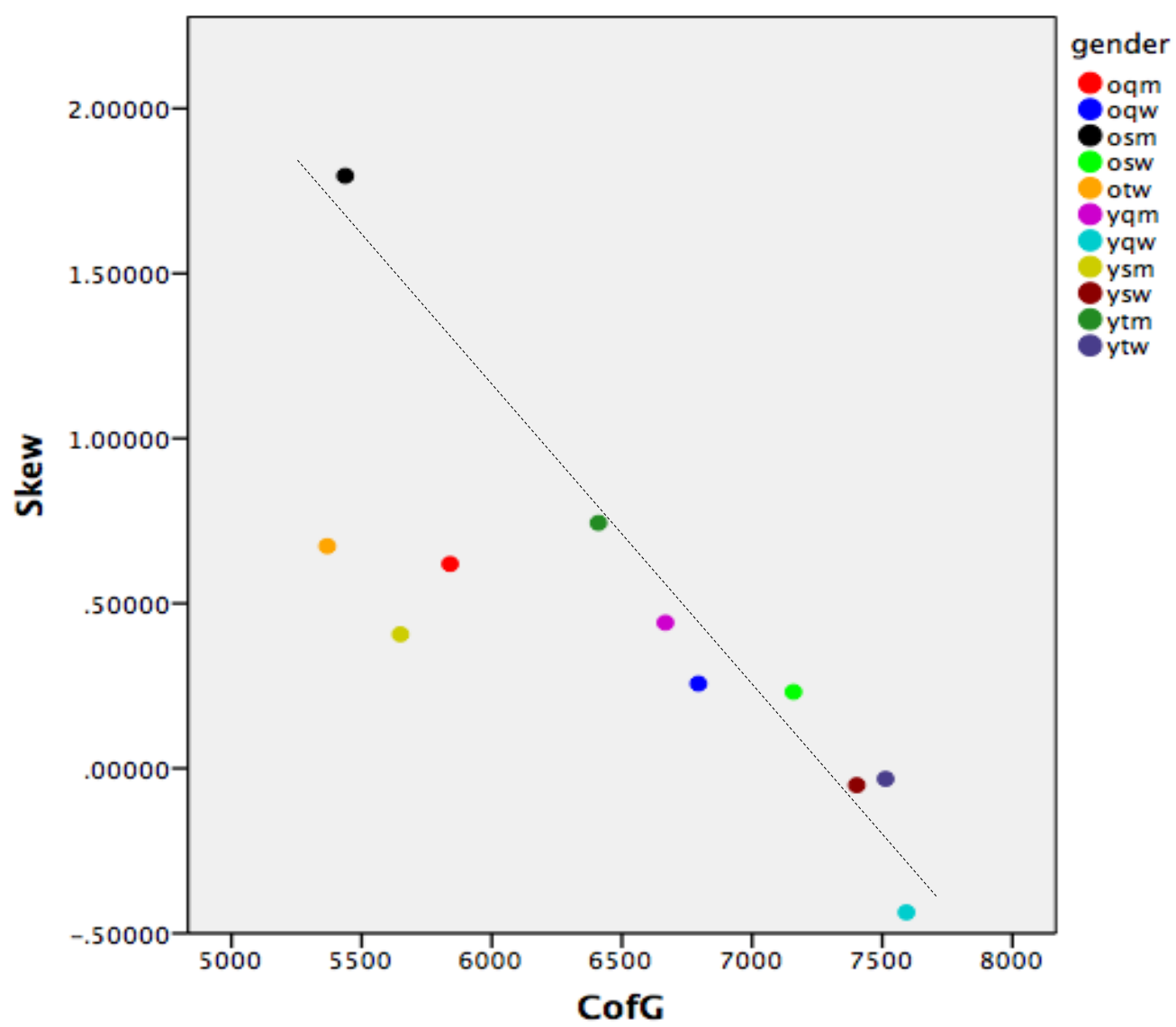

Figure 5.8: /s/ CofG vs. Skewness

\subsubsection{Means}

Tables 5.4 and 5.5 provide the means and standard deviations for the Centre of Gravity (CofG) and Skewness, respectively. These means provide a starting point for our discussion, as they give the broadest overview of the distribution for both measures. The relationship between CofG and Skewness is perhaps best visualised through a scatterplot (Figure 5.8).

We can see that, generally speaking, speaker groups with a higher CofG tend to have a lower Skewness, although there is some variation in the ordering of the groups. Younger speakers tend to have a higher CofG and lower Skewness than older ones, although this seems subordinated to the gender effect: women have a higher CofG and lower Skewness than men, with the clear exception of older trans women. They seem to be using /s/ as a locus for gendered presentation to a lesser extent than either of the younger trans groups, who are patterning much more in line with their identified gender.

As we noted in $§ 5.2 .6$, Skewness and CofG are predicted to be inversely related, and that is indeed largely what we see in Figure 5.8. Note that the dashed line on this graph represents an approximation of a direct linear relationship between CofG and Skewness, taking the highest-CofG/lowest-Skewness and the lowest-CofG/highest- 
Skewness as defining points (i.e., if the highest-CofG has the lowest-Skewness and vice versa, and if the relationship between the two is a linear one, then we would expect other points to fall along this line). Note that this line does not represent a statistical correlation, but rather a prediction of what a strictly linear relationship would be predicted to look like. Although the data shows a clear trend in the expected direction, the pattern is not a perfect fit. For the most part, speaker groups fall quite close to following this linear relationship, but there is a cluster of groups - older queer men, older trans women, and younger straight men - who fall below this line. That is to say, given how low their CofG is, their Skewness is lower than we would project. These three groups of speakers have connections with masculinity, but they are clearly oriented towards it in a different way than older straight men. Older queer men and younger straight men are invested in presenting a particular kind of masculinity, while older trans women may be continuing to produce tokens of $/ \mathrm{s} /$ more or less in line with what they would have been socialised to in their youth. These groups of speakers produce $/ \mathrm{s} /$ at the masculine end of the spectrum, but are able to differentiate themselves from a particular style of traditional heteronormative masculinity through their manipulation of skewness.

It is also interesting to note that speaker groups with a lower CofG tend to have a lower CofG standard deviation, particularly in the case of older straight men. There may be some effect of place of articulation in this: recall that lower frequencies are associated with more retracted articulations, and that as $/ \mathrm{s} /$ is retracted further and further, it begins to encroach on the place of articulation for $/ \mathrm{f} /$. This may mean that, for speakers with a very low CofG, there is relatively little room for variation without sacrificing perceptual clarity. However, this explanation is not entirely satisfactory, since while it may be true that there is a limit to how retracted /s/ can get for speakers with a very low overall CofG, it is does not follow that they cannot produce a more fronted variant. The degree of difference in Skewness between these older straight men and the groups with next highest Skewness measurements represents a substantial jump, which suggests that the production of /s/ by these older straight men is at least partly socially motivated.

\subsubsection{Homogeneous subsets}

The second analytical tool we will use to look at the distribution of CofG and Skewness is the homogeneous subsets derived from post-hoc Tukey tests following two ANOVAs (CofG: $\mathrm{F}(10,13739)=594.462, \mathrm{p}<0.001$; Skewness: $\mathrm{F}(10,13739)=511.65, \mathrm{p}<0.001)$. Tables 5.6 and 5.7 show which groups of speakers have means that are not significantly different from each other (constituting homogeneous subsets), and which are significantly different.

For both CofG and Skewness, there are several discrete homogeneous subgroups, although they are patterned differently between the two measurements. For 


\begin{tabular}{|c|c|c|c|c|c|c|c|c|c|}
\hline \multirow[b]{2}{*}{ Gender } & \multirow[b]{2}{*}{$N$} & \multicolumn{8}{|c|}{ Subset for alpha $=0.05$} \\
\hline & & 1 & 2 & 3 & 4 & 5 & 6 & 7 & 8 \\
\hline osm & 1505 & 5365 & & & & & & & \\
\hline otw & 869 & 5370 & & & & & & & \\
\hline ysm & 1349 & & 5661 & & & & & & \\
\hline oqm & 1334 & & & 5846 & & & & & \\
\hline ytm & 1567 & & & & 6424 & & & & \\
\hline yqm & 1492 & & & & & 6610 & & & \\
\hline oqw & 969 & & & & & & 6801 & & \\
\hline osw & 1365 & & & & & & & 7178 & \\
\hline ysw & 1481 & & & & & & & & 7425 \\
\hline ytw & 805 & & & & & & & & 7526 \\
\hline yqw & 1014 & & & & & & & & 7553 \\
\hline Sig. & & 1 & 1 & 1 & 1 & 1 & 1 & 1 & 0.249 \\
\hline \multicolumn{10}{|c|}{$\begin{array}{l}\text { Means for groups in homogeneous subsets are displayed. } \\
{ }^{a} \text { Uses Harmonic Mean Sample Size }=1184.992 . \\
{ }^{b} \text { The group sizes are unequal. The harmonic mean of the group sizes is used. Type I error } \\
\text { levels are not guaranteed. }\end{array}$} \\
\hline
\end{tabular}

\begin{tabular}{|c|c|c|c|c|c|c|c|c|}
\hline \multicolumn{9}{|c|}{ Table 5.7: /s/ Skewness: Homogeneous subsets (Tukey HSD ${ }^{\mathrm{a}, \mathrm{b}}$ ) } \\
\hline \multirow[b]{2}{*}{ Gender } & \multirow[b]{2}{*}{$N$} & \multicolumn{7}{|c|}{ Subset for alpha $=0.05$} \\
\hline & & 1 & 2 & 3 & 4 & 5 & 6 & 7 \\
\hline osm & 1505 & & & & & & & 1.8092 \\
\hline ytm & 1567 & & & & & & 0.7404 & \\
\hline otw & 869 & & & & & 0.6629 & 0.6629 & \\
\hline oqm & 1334 & & & & & 0.6049 & & \\
\hline yqm & 1492 & & & & 0.4415 & & & \\
\hline ysm & 1349 & & & & 0.3968 & & & \\
\hline oqw & 969 & & & 0.2193 & & & & \\
\hline osw & 1365 & & & 0.2159 & & & & \\
\hline ytw & 805 & & -0.0422 & & & & & \\
\hline ysw & 1481 & & -0.0726 & & & & & \\
\hline yqw & 1014 & -0.4574 & & & & & & \\
\hline Sig. & & 1 & 0.999 & 1 & 0.987 & 0.921 & 0.648 & 1 \\
\hline \multicolumn{9}{|c|}{$\begin{array}{l}{ }^{a} \text { Uses Harmonic Mean Sample Size }=1184.992 . \\
{ }^{b} \text { The group sizes are unequal. The harmonic mean of the group sizes is used. Type I error } \\
\text { levels are not guaranteed. }\end{array}$} \\
\hline
\end{tabular}


CofG, there are two clusters (older straight men and older trans women at one extreme; and younger straight, trans, and queer women at the other), and the remaining groups are all significantly different from each other. For Skewness, the picture is a bit different. The two extremes (older straight men and younger queer women) are significantly different from the adjacent groups, but all of the interior groups are divided into different subsets. For the most part, there is clear separation between these subsets: younger trans and straight women are not significantly different from each other, but they are from everyone else; similarly older queer and straight women, and younger queer and straight men. The remaining three groups are a bit more complicated: younger trans men are significantly different older queer men, but older trans women are not significantly different from either group. This is accounted for by the overlap between subsets 5 and 6 in Table 5.7 .

What these two tables tell us is that differences in CofG are more clear-cut than those that we find in Skewness. Both measures show clear trends that seem to reflect some degree of gendered identity, and although the patterns are very similar, the fact that they are not identical again reinforces the fact that they are independently manipulable, at least for the interior groups. We also see that younger trans speakers are producing variants that are more closely in line with their identified genders than the older trans women are, which raises the possibility that $/ \mathrm{s} /$ is a more heavily gendered marker among younger speakers than among older ones; however, these values are raw measurements, in the sense that they do not take any other factors into consideration, so these values may be distorted. We will now turn to the multivariate analysis, to disentangle social effects from structural ones.

\subsection{2 /s/: Statistical modelling}

Because the thrust of this thesis is on gendered identity and linguistic variation, the discussion of the multivariate analysis will focus principally on the social constraints. However, because of the way that multivariate analysis works in variationist sociolinguistics (e.g. Johnson 2009), linguistic factors were also taken into account, in order to more faithfully account for the observed variation. These linguistic factors will be discussed briefly in very general terms, before more attention is paid to the social factors.

It should also be noted that, for models of $/ \mathrm{s} /$ as well as for those of the other sibilants, the numbers of tokens presented in the multivariate analysis may differ from those reported elsewhere, due to necessary exclusions. It is the nature of a multivariate statistical package such as Rbrul that highly unevenly distributed data will produce unreliable models, so the data set had to be adjusted in some cases. Where possible, and where it was phonologically sound, tokens in very unbalanced cells were recoded so they could still be retained in the analysis; however, in some cases, it was more consistent to remove them altogether. 
Table 5.8: Multivariate analysis of /s/ CofG (social factors only) organised by age

\begin{tabular}{|c|c|c|c|c|c|}
\hline & & & $\begin{array}{r}\text { Total } \mathrm{N}^{\mathrm{a}} \\
\text { Log likelihood } \\
\text { Intercept }\end{array}$ & $\begin{array}{l}13750 \\
-114765.9 \\
6428.854\end{array}$ & \\
\hline (younger) & Coef & Mean & (older) & Coef & Mean \\
\hline trans women & 1068.422 & 7526 & straight women & 588.989 & 7178 \\
\hline queer women & 957.737 & 7553 & queer women & 272.658 & 6801 \\
\hline straight women & 933.448 & 7425 & queer men & -630.086 & 5846 \\
\hline queer men & 13.923 & 6610 & trans women & -1036.367 & 5370 \\
\hline trans men & -113.754 & 6424 & straight men & -1174.902 & 5365 \\
\hline straight men & -880.069 & 5661 & & & \\
\hline Range & 1948.491 & & Range & 1763.891 & \\
\hline \multicolumn{5}{|c|}{ Range overall: 2243.324} & \\
\hline \multicolumn{6}{|c|}{$\begin{array}{l}\text { Also retained as significant in model: } \\
\text { - Position in word }(\mathrm{p}<0.001) \\
\text { - Preceding segment: manner }(\mathrm{p}<0.001) \\
\text { - Preceding segment: place }(\mathrm{p}<0.001) \\
\text { - Following segment: place }(\mathrm{p}<0.001) \\
{ }^{a} N \text { and Means mav differ slightlv from previous tables due to exclusions }\end{array}$} \\
\hline $\begin{array}{l}{ }^{a} N \text { and Means } m c \\
\text { necessary for stat }\end{array}$ & $\begin{array}{l}y \text { differ slig } \\
\text { stical mode }\end{array}$ & $\begin{array}{l}\text { tly from } \\
\text { ing. }\end{array}$ & previous tables due $t$ & o exclusions & \\
\hline
\end{tabular}

Table 5.9: Multivariate analysis of /s/ Skewness (social factors only) organised by age

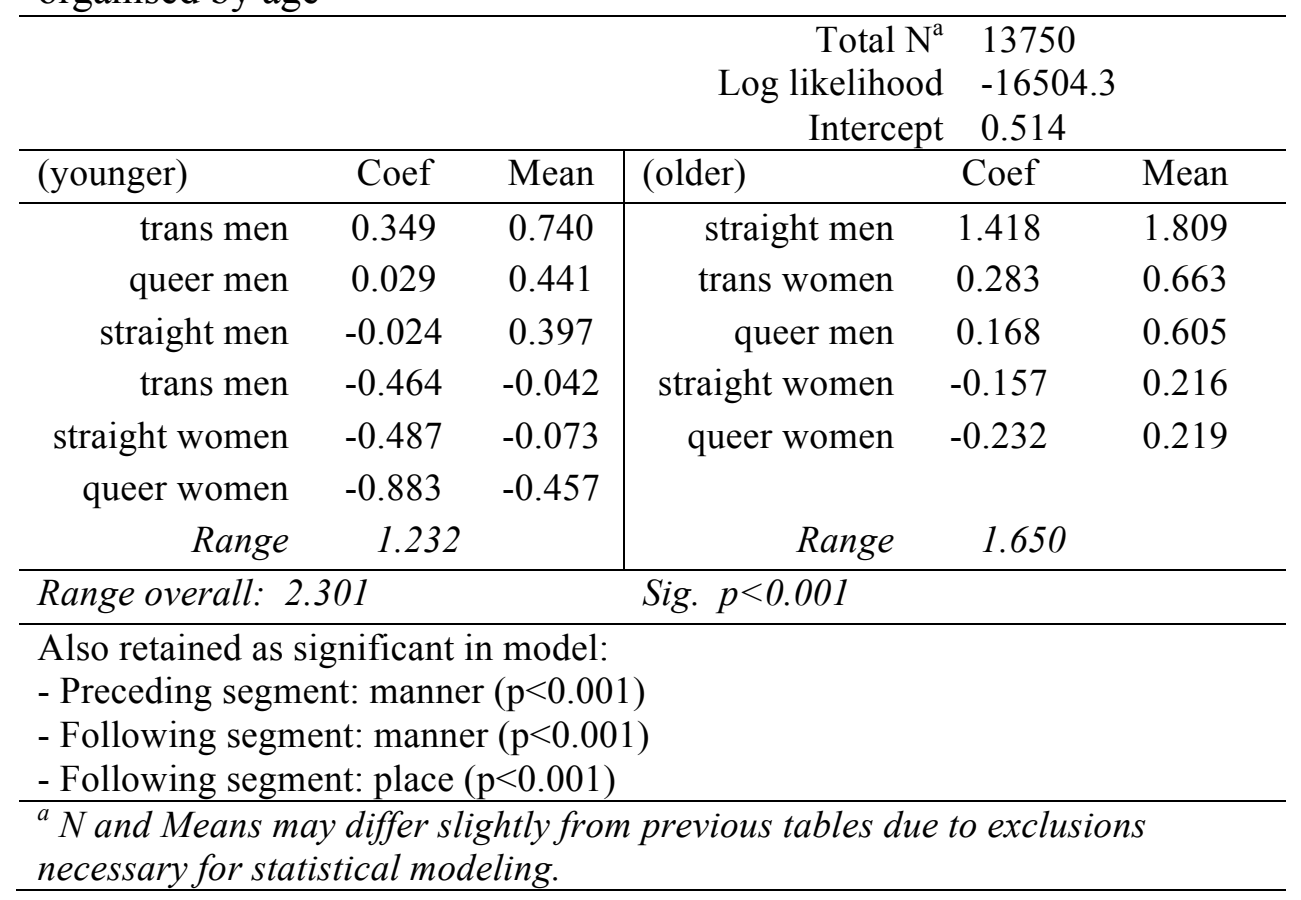


The linguistic factors taken into account in the statistical models were the position of the token in the word (initial, medial, or final), and the preceding and following segments, coded according to place and manner of articulation. Because the data is drawn from spontaneous speech (or as near to spontaneous speech as is possible with a sociolinguistic interview), it was not possible to control for the phonetic context of the tokens, so the data is highly variable. A number of coding options were trialed, and the one that seemed to most consistently produce reliable results was to code place of articulation as front, central, or back; and to code manner of articulation as either vowel or consonant; more fine-grained coding schema proved problematic for the statistical model, no doubt due to the large number of social factors being tested. Word boundaries were ignored with respect to identifying the preceding and following segments, unless that segment was an audible pause, which was coded separately for both place and manner. The majority of tokens that were removed from analysis (particularly in the multivariate analysis of $/ \mathrm{J} /$ that follows, in section $\$ 5.7 .2$ ) were preor post-pausal.

Tables 5.8 and 5.9 provide the results of the social factors for CofG and Skewness respectively, and also list a summary of the other factors retained as significant. Note that speaker was included as a random effect in all statistical models.

\subsubsection{Linguistic constraints}

Although both the preceding and following phonetic contexts are relevant for CofG and Skewness, it is interesting to note that they do not seem to be entirely balanced. The measurement of CofG seems to be more sensitive to the preceding segment, while Skewness seems more sensitive to the following segment. CofG also seems more sensitive to the place of articulation of the following and preceding segments, and Skewness to the manner. The model for CofG has also retained position in the word, while Skewness has not. These different sets of constraints may reflect the different articulatory parameters that the two measurements are sensitive to.

\subsubsection{Social constraints}

Statistical models were constructed that used the eleven-way gender categorisation shown here, as well as with the three dimensions considered independently; the model with the eleven categories was shown to be significantly better, by log-likelihood test. Tables 5.8 and 5.9 have been organised by age group, but this has been done for convenience and to make it easier to see the overall patterns in the data. The analysis itself was conducted with all eleven groups treated equally.

Among younger speakers, women produce /s/s that have much higher CofG than men do - and therefore more fronted articulations; the three groups of women are clustered quite close together, both in terms of their overall means as well as the coefficients that have been calculated (which indicate the effect that their gendered 
identity has on their production relative to other groups, all other factors being held constant). The FW for queer and trans men are relatively neutral, while straight men have a much lower production than their peers. Trans speakers pattern with their identified genders: trans women are closely matched to their cissexual peers, and trans men pattern quite closely with queer men; given that these men maintain close ties with queer communities and queer networks, this is not entirely surprising. The picture is a bit more complicated with skewness, where we still have women at one end and men at the other, but the coefficients are ranked differently. Here we see that younger trans men have the lowest skewness by a considerable margin, and that queer and straight men are patterning in very similar ways. We also see that queer women are doing something different to trans and straight women, with a considerably lower skewness.

Among older speakers, the general pattern holds, with women tending to produce more fronted variants than men, although older trans women continue to produce extremely backed variants, being very closely matched to older straight men in particular, in both mean CofG and coefficients. It is interesting to note that the spread of CofG coefficients for older speakers is less clustered than what we saw for younger groups, although the coefficients for skewness are clustered for older speakers as well. It may be that subtle differences in identity are more interpretable among older participants, particularly for the women: while the groups of younger women do not differentiate between themselves through place of articulation (although the lack of clear articulatory correlate for skewness is a frustration in this analysis), older women may be taking advantage of this dimension of /s/ production to signal their sexuality. With both age groups, however, the differences between groups of women are smaller than the differences between men and women.

\subsubsection{Discussion: /s/ as a site for gendered identity}

Gendered identity is clearly a relevant factor in both the CofG and skewness of /s/ in NZE. Young women (straight, queer, and trans) and older straight men occupy two ends of a continuum, with the other types of masculine and feminine identities more or less positioned with respect to these extremes. Older straight men are certainly producing an /s/ that seems less gradiently-linked to those of other identities, but this is large part due to the \{older trans women, older queer men, younger straight men\} cluster who are have an unexpectedly low Skewness, given their CofG (recall the scatterplot of Figure 5.8). This cluster marks a deviation from the strictly linear relationship between Skewness and CofG that we would expect all other things being equal, which suggests that these two spectral moments are independently manipulable, at least towards the centre of the $/ \mathrm{s} / \mathrm{spectrum}$. The fact that the identities in this cluster can be argued to have some degree of investment in, or experience with, a [+masculine] identity strongly suggests that this manipulation of one (or both) of these spectral moments is a site for gendered identity performance. 
There may be articulatory restrictions on how independent these two measures are at the extreme ends of the /s/ continuum, because the phonetic cues to place of articulation can only overlap with adjacent fricatives so much before there is a strong risk of the segment being mis-heard by listeners. A too-fronted $/ \mathrm{s} / \mathrm{may}$ begin to sound like a $/ \theta /$ (sin vs $\underline{\boldsymbol{t}} \boldsymbol{i}$ in), while a too-retracted one may begin to sound like $/ \int /$ ( $\underline{\sin }$ vs $\underline{\text { sh }}$ in). Towards these two extreme ends, it may be perceptually important that CofG and Skewness interact as expected; but towards the centre of the spectrum, there may be more freedom in how these two measures, or rather the articulatory correlates of these two measures, are put into practice.

We have noted that one end of the /s/ continuum seems to be masculinemarked, while the other is feminine-marked: [+masculine] and [+feminine], respectively. This suggests that these two broad categories are somehow continuous, that it is possible to get from [ + masculine $]$ to $[+$ feminine $]$ through a finite number of discrete steps. It is not immediately clear that this is the case. It also suggests that the midpoint of this continuum should be neither masculine nor feminine, but rather some kind of agendered zero through which one must pass on the journey from one extreme to the other; this is likewise not a particularly satisfying position to take.

For one thing, such a simple continuum does not bear much resemblance to our experience of the world: we know many people who align with different styles of masculinity and femininity, but we probably know very few (if any) people who identify as completely androgynous, neither masculine nor feminine in any way. It could be that this central space is simply underpopulated, and that people tend to cluster to one side or the other of this midpoint, but this does away with the analytical lens of thinking about continua at all. Secondly, it would necessitate that anyone who was positioned on the 'wrong' side of this midpoint be classed as transgender or transsexual. If we recall from Figure 5.8 (the scatterplot of CofG and Skewness for $/ \mathrm{s} /$ ), younger queer men were positioned closer to the [+feminine] end of our hypothetical continuum, but they identify as men, not as trans women.

So one simple continuum of [ + masculine $]$ to $[+$ feminine $]$ seems unlikely. It may be more productive to consider parallel continua, then; one that indexes femininity, and a separate one that indexes masculinity, as in Figure 5.9. Under this

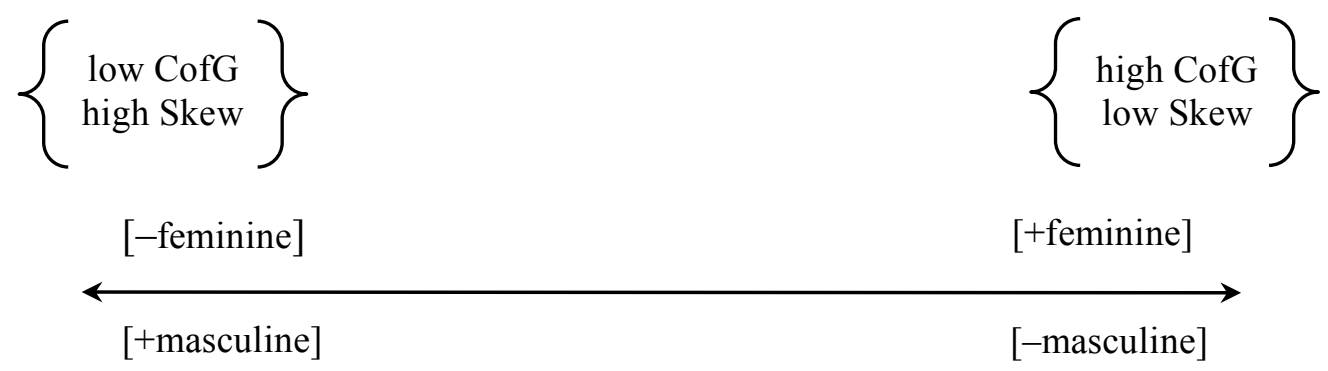

Figure 5.9: Parallel continua for gendered indexicality of $/ \mathrm{s} /$ 
proposed scheme, people who present as (or are interpreted as) men would be evaluated along one of these continua, and those who present as women would be evaluated along the other. Indexical bleed-through between these two scales could also account for some of the stereotypes about the speech of gay men, in that their articulatory gestures might position them towards the [-masculine] end of the men's scale, which would then be interpretable as overlapping with the [+feminine] end of the corresponding women's scale. But what would the social meanings associated with the extremes of these scales be? And what would it mean to be positioned at the negative end of them?

We could take the position that [masculine] refers to a cluster of positivelyevaluated traits that are stereotypically associated with men and masculinity (things such as assertiveness, confidence, power, strength), while [feminine] refers to a complementary cluster of positively-evaluated traits that stereotypically mark femininity (such as nurturing, emotional openness, empathy). The [+masculine] end would then be an embodiment or performance of these positively-evaluated traits, and the [-masculine] end a rejection or distancing from these traits, and the same with [+] and [-femininity].

But this also does not bear up to close scrutiny, because it implies that nonstereotypically-heteronormative men are in some way rejecting the positively-evaluated aspects of masculinity, and that non-stereotypically-heteronormative women are likewise rejecting the positively-evaluated aspects of femininity. This does not seem likely, or indeed justified: if [+feminine] in this case aligns with nurturing and empathy, then we are necessarily saying that some women - such as older queer women, in this data - are enacting femininities that are in opposition to these qualities. Given that several of these participants in the ATC have children and enjoy motherhood, this does not seem to be a fair assessment of their identities.

Perhaps, then, rather than thinking of the continua as ranging between embodiment or rejection of one bundle of associations, it is more productive to think about two contrasting styles of masculinity and femininity, and anchor the endpoints of the continuum to these complementary but related sets of associations (Figure 5.10). This schema also allows for some crossover of associations - if masculinity B and femininity B share certain traits, for example - but does not entail that people are necessarily opting out of all of the social qualities associated with masculinity or

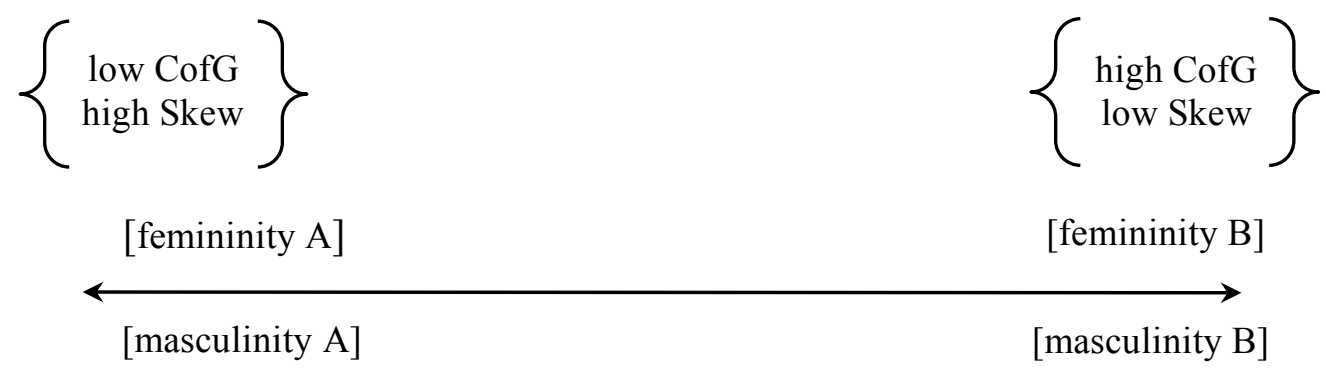

Figure 5.10: Revised parallel continua for gendered indexicality of /s/ 
femininity by rejecting one particular form of gendered expression.

Of course, at this point, we do not have a strong sense of what qualities should make up either of the styles of femininity or masculinity, but this approach at least provides a framework for interpreting data which seems, on the surface, to present us with a continuum of gendered expression. This model will be explored in greater detail in $\$ 5.8 .1$, as well as in chapter eight where we begin to consider patterns across multiple variables.

First, however, we turn our attention to the other sibilants in this chapter: $/ \mathrm{z} /$ and $/ \mathrm{S} /$.

\section{$5.6 \quad \mid z /$}

The difference between $/ \mathrm{s} /$ and $/ \mathrm{z} /$, in principle, is simply one of voicing: the two segments are otherwise considered to have the same phonetic properties. There are phonological differences, of course $-[\mathrm{s}]$ is an allophone of $/+\mathrm{z} /$ suffixes that follow unvoiced segments - which means that [s] and [z] will necessarily have somewhat different distributions with respect to phonetic environments. But because this study uses spontaneous speech, phonetic environment includes segments that straddle word boundaries, so the differences between the phonetic environments of $/ \mathrm{s} /$ and $/ \mathrm{z} /$ will likely not be categorical, but rather a question of degree. That is to say, $/ \mathrm{z} /$ will likely be preceded by more voiced segments than $/ \mathrm{s} /$, but potential word combinations such as that zoo and dog snarling mean that it is possible for there to be a voicing mismatch with the preceding segment.

But what is the effect that the voicing will have on our analysis? After all, one of the reasons that we didn't have to normalise for biological differences with $/ \mathrm{s} / \mathrm{is}$ that it was voiceless, meaning that the laryngeal differences between adult men and women were irrelevant to the measurements we were taking. In the analysis of vowels (see chapter six), all of the formant values are normalised to eliminate the effect of biology, and to allow the discussion to focus on the social factors. Does the voicing of $/ \mathrm{z} /$ mean that we have to adopt a similar approach?

As it turns out, we do not. With vowels, the formants represent the frequencies at which there is resonance in the vocal tract, amplifying certain harmonics of the fundamental frequency ( $\mathrm{F} 0$, or more generally, the pitch of a person's normal speaking voice). Vowels are produced with little to no constriction of the air flow: the tongue, jaw, and lips direct the air in ways that create resonance at particular frequencies (different combinations of which we perceive as different vowel sounds), but the flow of air is otherwise undisturbed.

In contrast, fricatives - particularly sibilant fricatives - rely on constricting the air flow enough to producing a hissing sound at frequencies generally well above F0; 
Table 5.10: /z/ CofG - Means and Standard Deviations (Hz)

\begin{tabular}{cccc}
\hline Gender & $N$ & Mean & St.Dev. \\
\hline ysm & 741 & 4577 & 1664 \\
otw & 553 & 4716 & 1339 \\
osm & 812 & 5022 & 942 \\
oqm & 689 & 5057 & 1525 \\
ytm & 866 & 5864 & 1562 \\
yqm & 842 & 6125 & 1610 \\
oqw & 503 & 6290 & 1438 \\
ysw & 859 & 6378 & 1785 \\
ytw & 370 & 6437 & 1950 \\
osw & 787 & 6780 & 1379 \\
yqw & 515 & 6915 & 1731 \\
\hline
\end{tabular}

Table 5.11: /z/ Skewness - Means and Standard Deviations (units of Skew)

\begin{tabular}{cccc}
\hline Gender & $N$ & Mean & St.Dev. \\
\hline osm & 812 & 1.87167 & 1.40952 \\
ysm & 741 & 0.97367 & 1.22963 \\
otw & 553 & 0.85824 & 1.05677 \\
oqm & 689 & 0.69396 & 0.81743 \\
ytm & 866 & 0.65540 & 0.90398 \\
ytw & 370 & 0.50169 & 1.19981 \\
yqm & 842 & 0.36908 & 0.77670 \\
oqw & 503 & 0.31203 & 0.98140 \\
ysw & 859 & 0.10310 & 1.13130 \\
osw & 787 & 0.08880 & 1.07625 \\
yqw & 515 & -0.56387 & 1.08955 \\
\hline
\end{tabular}




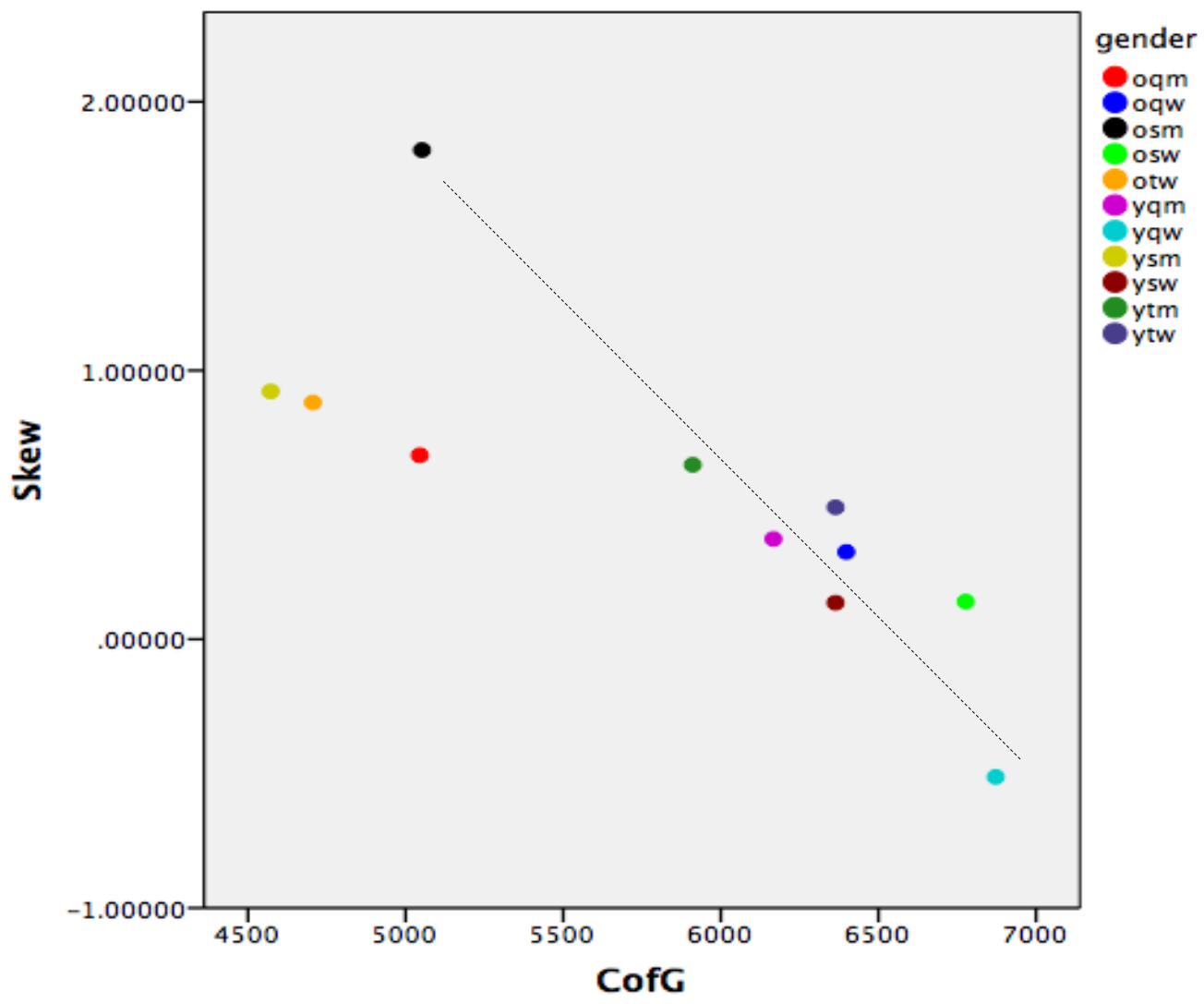

Figure 5.11: /z/ CofG vs. Skewness

adult males tend to have $\mathrm{F} 0$ in the range of $107-118 \mathrm{~Hz}$, and adult females tend to have F0 in the range of 184-205 Hz (e.g., Gelfer \& Bennett 2013), while the frequencies that we have been considering for place of articulation of $/ \mathrm{s} /$, for example, are above 5000 Hz. In fricative-type constrictions, the oral tract does not resonate, and there are no formants to contend with in the analysis of fricatives. The high-pass filter applied to the sound signal before the spectral moments are measured excludes any frequencies below $500 \mathrm{~Hz}$, so that the energy concentration corresponding to F0 does not interfere with the measurement of frication. Hayward (2000: 192) notes that "the overall pattern [for voiced fricatives] will be similar to that for the corresponding voiceless fricatives, but the intensity of the friction will be weaker." Since intensity is not one of the acoustic properties we are examining here, this will not pose a problem for comparability of $/ \mathrm{z}$ / and $/ \mathrm{s} /$.

\subsection{1 /z/: Distribution}

\subsubsection{Means}

The means for Centre of Gravity (CofG) and Skewness are provided in Tables 5.10 and 5.11. From a gendered identity perspective, the pattern with $/ z /$ is similar to what we 


\begin{tabular}{cccc}
\hline \multicolumn{4}{c}{ Table 5.12: /s/ and /z/ CofG means and } \\
difference between means (Hz) \\
\hline Gender & $/ s /$ & $/ z /$ & diff. \\
\hline osm & 5365 & 5022 & 343 \\
osw & 7178 & 6780 & 398 \\
yqm & 6610 & 6125 & 485 \\
oqw & 6801 & 6290 & 511 \\
ytm & 6424 & 5864 & 560 \\
yqw & 7553 & 6915 & 638 \\
otw & 5370 & 4716 & 654 \\
oqm & 5864 & 5057 & 807 \\
ysw & 7425 & 6378 & 1047 \\
ysm & 5661 & 4577 & 1084 \\
ytw & 7526 & 6437 & 1089 \\
\hline
\end{tabular}

found for /s/: men generally have lower CofG and higher skewness than women, and younger speakers also tend to have a higher CofG and lower Skewness than older speakers. Older trans women are once again at the low-CofG end of the range, patterning more like older straight men (CofG) and older queer men (Skewness) than their women peers.

The scatterplot in Figure 5.11, which plots CofG against Skewness, also shows a pattern broadly similar to what we observed with $/ \mathrm{s} /$ : for the most part, there is an inverse correlation between CofG and Skewness, and the three speaker groups that lie off this line are older queer men, older trans women, and younger straight men; and again, these three groups cluster at the more masculine-dominated end of the correlational line.

What is interesting is that the means for $/ \mathrm{z} /$ are notably lower than they are for $/ \mathrm{s} /$. This is unexpected given that CofG is associated with place of articulation, and $/ \mathrm{s} /$ and $/ \mathrm{z} /$ should in principle have the same place of articulation. Table 5.12 provides the mean CofGs for $/ \mathrm{s} /$ and $/ \mathrm{z} /$, and the difference between these means, across speaker groups.

For some speakers, the frequency difference between the two phonemes is relatively small, although even $300 \mathrm{~Hz}$ should be detectable; but this may partly be due to the difference in phonetic environments mentioned. For other groups, however, the difference between the means is considerably larger, over $1000 \mathrm{~Hz}$, and it seems unlikely that such a difference can be solely accounted for by linguistic factors. We might try to make an argument that extremely fronted variants of /s/ (with a CofG over $7000 \mathrm{~Hz}$ ) are the outliers, and so the greater acoustic distance between $/ \mathrm{s} /$ and $/ \mathrm{z} /$ for younger straight and trans women is a product of $/ \mathrm{s} /$ rather than of $/ \mathrm{z} /$. However, younger straight men also have a difference of over $1000 \mathrm{~Hz}$ between /s/ and /z/, and they produce one of the lower-CofG variants of /s/; so the acoustic distance between /s/ 


\begin{tabular}{|c|c|c|c|c|c|c|c|}
\hline \multirow[b]{2}{*}{ Gender } & \multirow[b]{2}{*}{$N$} & \multicolumn{6}{|c|}{ Subset for alpha $=0.05$} \\
\hline & & 1 & 2 & 3 & 4 & 5 & 6 \\
\hline ysm & 741 & 4577 & & & & & \\
\hline otw & 553 & 4716 & & & & & \\
\hline osm & 812 & & 5022 & & & & \\
\hline oqm & 689 & & 5057 & & & & \\
\hline ytm & 866 & & & 5864 & & & \\
\hline yqm & 842 & & & 6125 & 6125 & & \\
\hline oqw & 503 & & & & 6290 & 6290 & \\
\hline ysw & 859 & & & & 6378 & 6378 & \\
\hline ytw & 370 & & & & & 6437 & \\
\hline osw & 787 & & & & & & 6780 \\
\hline yqw & 515 & & & & & & 6915 \\
\hline Sig & & 0.878 & & 0.088 & 0.111 & 0.833 & 0.899 \\
\hline
\end{tabular}

Means for groups in homogeneous subsets are displayed.

${ }^{a}$ Uses Harmonic Mean Sample Size $=637.665$.

${ }^{b}$ The group sizes are unequal. The harmonic mean of the group sizes is used. Type I error levels are not guaranteed.

\begin{tabular}{|c|c|c|c|c|c|c|c|c|}
\hline \multirow[b]{2}{*}{ Gender } & \multirow[b]{2}{*}{$N$} & \multicolumn{7}{|c|}{ Subset for alpha $=0.05$} \\
\hline & & 1 & 2 & 3 & 4 & 5 & 6 & 7 \\
\hline osm & 812 & & & & & & & 1.8717 \\
\hline ysm & 741 & & & & & & 0.9737 & \\
\hline otw & 553 & & & & & 0.8582 & 0.8582 & \\
\hline oqm & 689 & & & & 0.6940 & 0.6940 & & \\
\hline ytm & 866 & & & & 0.6554 & & & \\
\hline ytw & 370 & & & 0.5017 & 0.5017 & & & \\
\hline yqm & 842 & & & 0.3691 & & & & \\
\hline oqw & 503 & & & 0.3120 & & & & \\
\hline ysw & 859 & & 0.1031 & & & & & \\
\hline osw & 787 & & 0.0888 & & & & & \\
\hline yqw & 515 & -0.5639 & & & & & & \\
\hline Sig. & & 1 & 1 & 0.06 & 0.053 & 0.184 & 0.703 & 1 \\
\hline
\end{tabular}

Means for groups in homogeneous subsets are displayed.

${ }^{a}$ Uses Harmonic Mean Sample Size $=637.665$.

${ }^{b}$ The group sizes are unequal. The harmonic mean of the group sizes is used. Type I error levels are not guaranteed. 
and /z/ cannot be so simply explained.

Nor can we convincingly argue that the backing of / $\mathrm{z} /$ (if that is what is happening) is a change in progress. It is true that the three groups of speakers with the greatest CofG differences are younger, but there are older groups who also have a nontrivial separation. Furthermore, the patterns overall do not follow what we would predict for a change in progress. Sound changes typically either show young women leading in the use of the prestige variant in the case of changes from above (with some degree of conscious awareness), or leading in the use of the innovative variant in changes from below (e.g., Labov 2001). We would not expect to find young women, young men and older men to be at the leading edge. Similarly, if the change were in the opposite direction - that is to say, if the change was a narrowing of the gap between /s/ and $/ \mathrm{z} /$, then we would not expect older men, older women, and younger men to be in the lead. If this is not a change in progress, then perhaps this lowered CofG is characteristic of /z/ in New Zealand English. We will revisit the phonological implications of this in $\S 5.8$, below.

\subsubsection{Homogeneous subsets}

Tables 5.13 and 5.14 show the homogeneous subsets for Centre of Gravity and Skewness, following two ANOVAs (CofG: $F(10,7526)=197.007, p<0.001$; Skew: $\mathrm{F}(10,7526)=231.384, \mathrm{p}<0.001)$.

Skewness seems more gradient than CofG, in that there are more subsets in Table 5.14, and more overlap between adjacent subsets, at least for the non-extreme groups. The jumps between the highest and the second-highest - and between the lowest and the second-lowest - for Skewness are more abrupt than between the interior groups, and relatively more extreme than anything that we see with CofG; it may be more accurate, then, to note that CofG is more continuously gradient, while Skewness has extreme values at the upper and lower ends but is gradient between them.

\subsection{2 /z/: Statistical modelling}

As with /s/, the multivariate analysis reported here for $/ \mathrm{z} /$ focuses on the social constraints, although the broad findings of the linguistic constraints will be touched on briefly, as they were included in the model to tease out the gendered effects. In both Tables 5.15 and 5.16, the social groupings have been separated by age, but this was done after the analysis, and only for convenience; all speaker groups were tested together. We will touch briefly on the linguistic constraints before looking more closely at the social factors underlying the observed variation.

\subsubsection{Linguistic constraints}

As Table 5.15 shows, CofG for $/ \mathrm{z} /$ is more sensitive overall to the preceding segment than it is to the following, and more sensitive to place than to manner. This tallies with 
Table 5.15: Multivariate analysis of /z/ CofG (social factors only) organised by age

\begin{tabular}{|c|c|c|c|c|c|}
\hline & & & $\begin{array}{r}\text { Total } \mathrm{N}^{\mathrm{a}} \\
\text { Log likelihood } \\
\text { Intercept } \\
\end{array}$ & $\begin{array}{l}7510 \\
-65145.16 \\
5823.972 \\
\end{array}$ & \\
\hline (younger) & Coef & Mean & (older) & Coef & Mean \\
\hline queer women & 1000.404 & 6415 & straight women & 827.064 & 6776 \\
\hline trans women & 657.543 & 6442 & queer women & 395.143 & 6303 \\
\hline straight women & 605.664 & 6380 & queer men & -659.519 & 5058 \\
\hline queer men & 120.809 & 6125 & straight men & -809.878 & 5022 \\
\hline trans men & -16.800 & 5865 & trans women & -876.852 & 4708 \\
\hline straight men & -1243.587 & 4578 & & & \\
\hline Range & 2243.991 & & Range & 1703.916 & \\
\hline Range overall: 22 & 3.991 & & Sig. $p<0.001$ & & \\
\hline \multicolumn{6}{|c|}{$\begin{array}{l}\text { Also retained as significant in model: } \\
\text { - Following segment: place }(p<0.001) \\
\text { - Preceding segment: manner }(p<0.001) \\
\text { - Preceding segment: place }(p=0.024)\end{array}$} \\
\hline \multicolumn{6}{|c|}{$\begin{array}{l}{ }^{a} N \text { and Means may differ slightly from previous tables due to exclusions } \\
\text { necessary for statistical modeling. }\end{array}$} \\
\hline
\end{tabular}

Table 5.16: Multivariate analysis of / $z$ / Skewness (social factors only) organised by age

\begin{tabular}{|c|c|c|c|c|c|}
\hline & & & $\begin{array}{r}\text { Total } \mathrm{N}^{\mathrm{a}} \\
\text { Log likelihood } \\
\text { Intercept }\end{array}$ & $\begin{array}{l}7510 \\
-10365.5 \\
0.586\end{array}$ & \\
\hline (younger) & Coef & Mean & (older) & Coef & Mean \\
\hline straight men & 0.421 & 0.973 & straight men & 1.335 & 1.873 \\
\hline trans men & 0.175 & 0.655 & trans women & 0.200 & 0.860 \\
\hline trans women & -0.069 & 0.501 & queer men & 0.170 & 0.695 \\
\hline queer men & -0.155 & 0.368 & queer women & -0.241 & 0.305 \\
\hline straight women & -0.451 & 0.100 & straight women & -0.331 & 0.094 \\
\hline queer women & -1.055 & -0.564 & & & \\
\hline Range & 1.476 & & Range & 1.666 & \\
\hline Range overall: 2.39 & & & Sig. $p<0.01$ & & \\
\hline \multicolumn{6}{|c|}{$\begin{array}{l}\text { Also retained as significant in model: } \\
\text { - Following segment: manner }(p<0.001) \\
\text { - Following segment: place }(\mathrm{p}<0.001)\end{array}$} \\
\hline \multicolumn{6}{|c|}{$\begin{array}{l}{ }^{a} N \text { and Means may differ slightly from previous tables due to exclusions } \\
\text { necessary for statistical modeling. }\end{array}$} \\
\hline
\end{tabular}


what we observed for $/ \mathrm{s} /$ as well. Similarly, the Skewness for $/ \mathrm{z} /$ is more sensitive to the following environment (Table 5.16), although manner does not seem to be more influential than place in this case.

Generally speaking, /s/ and / $/$ / seem largely affected by the same sets of linguistic factors, although a closer examination of these patterns may note differences; however, such a closer phonetic discussion is beyond the scope of this project. It is enough to note that the two segments - nominally differing only in voicing, despite our evidence that there is also a degree of variation in place of articulation - are constrained by broadly parallel systems of structural factors.

\subsubsection{Social constraints}

As we might expect, given our descriptive observations, we see a familiar gender split in both age groups: women tend to have higher CofG and lower Skewness than men, although with /z/, the range of coefficients spanned by younger speakers extends above and below that spanned by older speakers. Recall that the coefficients make the different groups comparable with the category of gendered identity, by levelling the effect of other linguistic factors. This means that, although the overall mean CofG for older straight women is higher than that of younger queer women, when the effect of the phonetic environment is accounted in the analysis, younger queer women actually have a tendency to produce /z/ with a higher CofG than older straight women.

Among younger speakers, queer women produce /z/ with the highest CofG generally (evidenced by their coefficient, which is substantially higher than the nexthighest), and straight men the lowest; queer and trans men show no particularly strong effect of gender, while trans women and straight women have a moderately raising effect on CofG. The magnitude of straight men's coefficient relative to their nearest neighbour is interesting, suggesting that retraction of $/ z /$ is much more pronounced among these men than among any of their peers, although it is interesting to note that the jump between older straight men and younger straight men is much less pronounced. Younger men are producing / $\mathrm{z} /$ with a CofG more comparable to their older counterparts than to their peers. With Skewness, queer women have the strongest lowering effect, and straight men have the highest raising effect, and the interior groups are fairly evenly spread out, showing little clustering. Generally, speakers with higher CofG coefficient have a lower Skewness coefficient and vice versa, but younger trans women are reordered relative to the other groups of speakers, and are patterning more like young men with Skewness, despite being much closer to the other young women with CofG. Trans men pattern with cis men consistently across both measures.

Among older speakers, women also have higher CofGs and lower Skewness than men, with straight women producing a substantially higher CofG (with respect to coefficients) than queer women, although the difference between their Skewness coefficients is much less pronounced. Queer men, straight men, and trans women 
pattern closely with respect to CofG, although straight men deviate from this masculine cluster with Skewness by quite a lot. The coefficients for Skewness show a strong drop between straight men and trans women, and then the changes are slightly more incremental from group to group. Trans women are patterning on par with straight men for CofG, and queer men for Skewness, suggesting that they are not using $/ \mathrm{z} /$ as a resource for gendered presentation.

\subsubsection{Discussion: / $z /$ as a site for gendered identity}

Despite showing a similar overall pattern to what we saw with $/ \mathrm{s} /, / \mathrm{z} /$ is not part of the linguistic stereotypes of either young women or gay men, suggesting a lower degree of social salience. However, the CofG findings for the younger trans speakers suggests that it is carrying some degree of indexicality, albeit a less consciouslyaccessible one. This social difference between $/ \mathrm{s} /$ and $/ \mathrm{z} /$ may be attributable at least in part to what seems to be a difference in place of articulation, with $/ z /$ being more retracted than $/ \mathrm{s} /$ (or $/ \mathrm{s} /$ being more fronted than $/ \mathrm{z} /$ ).

The broad similarities with respect to social and linguistic factors, coupled with the IPA classification of $/ \mathrm{s} /$ and $/ \mathrm{z} /$ as both being alveolar fricatives, makes it seem that the /s, z/ system has been split along voicing lines, with either/s/ pushed forward or /z/ retracted. A consequence of $/ \mathrm{s} /$ having a further forward place of articulation is that it is being produced closer to the teeth than $/ \mathrm{z} /$, and the teeth are the source of the secondary airflow turbulence that characterises the sibilants. Social differences may then flow from this proximity, with the perceptual salience of this secondary turbulence being the primary index of gendered identity. Following this train of thought to its end, in fact, raises the possibility that the reason that /s/ gets fronted by women is specifically to take advantage of this secondary turbulence, as a modifier of the sound signal that carries social information.

With $/ z /$ being more retracted and therefore having a less dominant secondary turbulence in the sound signal, the gendered salience that it carries may fall below the threshold of conscious awareness. It is worth noting that although this secondary turbulence may in fact be causing an exaggeration in the CofGs for $/ \mathrm{s} /$ that is unrelated to place of articulation (making the difference between $/ \mathrm{s} /$ and $/ \mathrm{z} /$ seem larger than it is), it is unlikely that this exaggeration would be of the magnitude that we observed when we compared mean CofGs. So while it is possible that the scale of difference is somewhat distorted, this does not mean that there is no difference in place of articulation between $/ \mathrm{s} /$ and $/ \mathrm{z} /$. Recall from Table 5.12 that younger straight men (whose CofG for/s/ was low enough as to be unaffected as strongly by any secondary turbulent effect) had one of the biggest CofG differences between $/ \mathrm{s} /$ and $/ \mathrm{z} /$. If this difference were completely attributable to the frontness of $/ \mathrm{s} /$, then we would not see this kind of pattern; we would not expect to see their $/ \mathrm{z} / \mathrm{CofG}$ as far retracted as it is.

Based on this data, /s/ and $/ \mathrm{z} /$ share the same basic distribution, but have shifted 
in place of articulation. This difference in proximity to the source of the secondary turbulence has resulted in $/ \mathrm{s} /$ carrying accessible gendered cues (as seen in stereotypes of particular styles of gendered identity) and /z/ falling below the radar. However, the same pattern underlies both the distributions, and we see the same behaviour in trans speakers with respect to both sibilants, which suggests that $/ z /$ does do identity work, but at a consciously inaccessible level. Because $/ \mathrm{s} /$ and $/ \mathrm{z} /$ canonically differ only in voicing, it is worth asking whether $/ z /$ shows this pattern because of gender, or if it gets the gendered effect for free (as it were) because it is so closely related to $/ \mathrm{s} /$. Given the strong effect of voicing on the perception of gender (previous studies of sibilants - e.g., Munson \& Coyne 2010; Gordon, Barthmaier \& Sands 2002; Fox \& Nissen 2005 - have tended to examine only voiceless segments), there is a strong possibility that the perceptual cues provided by F0 swamp anything happening in the range of frequencies associated with frication. Additionally, $/ \mathrm{s} /$ is a generally noisier fricative than $/ \mathrm{z} /-$ recall from \$5.3.4 that Hayward (2000: 192) points out that voiced fricatives generally have a weaker intensity than their voiceless counterparts. This difference may also render $/ z /$ consciously uninterpretable. Perceptual studies to examine the gendered salience of $/ z /$ would be required to determine the degree of socially interpretable meaning that $/ \mathrm{z} /$ carries, which would be an interesting question to explore but is not part of this study; it will provide fertile ground for future research into the sociophonetics of English sibilants.

\section{$5.7 / \mathrm{d} /$}

Our discussion of $/ \mathrm{s} /$ looked at realisations as being more $/ \mathrm{s} /$-like (fronter articulations) or more $/ \mathrm{J} /$-like (more backed realisations), in that retracted tokens of $/ \mathrm{s} /$ have spectral characteristics that begin to approximate those of $/ \mathrm{J} /$. We also saw that $/ \mathrm{z} /$ has a seemingly more backed articulation than we might expect given our observations on $/ \mathrm{s} /$, meaning that $/ \mathrm{z} /$ is closer in Centre of Gravity (CofG) to $/ \mathrm{J} /$ than $/ \mathrm{s} /$ is. $/ \mathrm{s} /$ carries overt social meaning, while $/ z /$ seems not to, or at least not to the extent that is has become part of a conscious gendered repertoire (i.e., $/ z /$ is not part of gendered stereotypes in the way that $/ \mathrm{s} /$ is). We have explored the possibility that the gendered indexicality of sibilants is related to their place of articulation, potentially mediated by the effect of secondary turbulence to the airstream.

How then does $/ \mathrm{g} /$ fit into this phonetic and social space? It has a more retracted place of articulation, which may make it less available for gendered identity work generally. On the other hand, it is relatively unbounded, in the sense that there is no English fricative closely spaced behind it (notwithstanding varieties that have retroflex or velar fricatives - neither of which is present in Auckland English - the next fricative back is $/ \mathrm{h} /$ ). Recall that $/ \mathrm{s} /$ can only be retracted so far before it approximates 
Table 5.17: / $/$ CofG - Means and Standard Deviations $(\mathrm{Hz})$

\begin{tabular}{cccc}
\hline Gender & $N$ & Mean & St.Dev. \\
\hline oqm & 158 & 3631 & 760 \\
ysm & 152 & 3755 & 686 \\
osm & 184 & 3770 & 853 \\
osw & 284 & 3971 & 653 \\
otw & 174 & 3981 & 797 \\
yqm & 200 & 4043 & 713 \\
ytm & 196 & 4066 & 1017 \\
oqw & 155 & 4219 & 821 \\
ytw & 105 & 4305 & 737 \\
ysw & 199 & 4449 & 805 \\
yqw & 159 & 4485 & 803 \\
\hline
\end{tabular}

Table 5.18: / $/$ / Skewness - Means and Standard Deviations (units of Skew)

\begin{tabular}{cccc}
\hline Gender & $N$ & Mean & St.Dev. \\
\hline osm & 184 & 2.79712 & 1.91268 \\
ytm & 196 & 1.99539 & 1.44794 \\
oqm & 158 & 1.84782 & 0.86241 \\
yqw & 159 & 1.79172 & 1.1967 \\
yqm & 200 & 1.71208 & 1.00543 \\
osw & 284 & 1.67678 & 1.16302 \\
ytw & 105 & 1.64668 & 1.13554 \\
oqw & 155 & 1.43414 & 0.81816 \\
ysm & 152 & 1.43375 & 0.59887 \\
ysw & 199 & 1.39058 & 0.81653 \\
otw & 174 & 1.14353 & 0.96644 \\
\hline
\end{tabular}




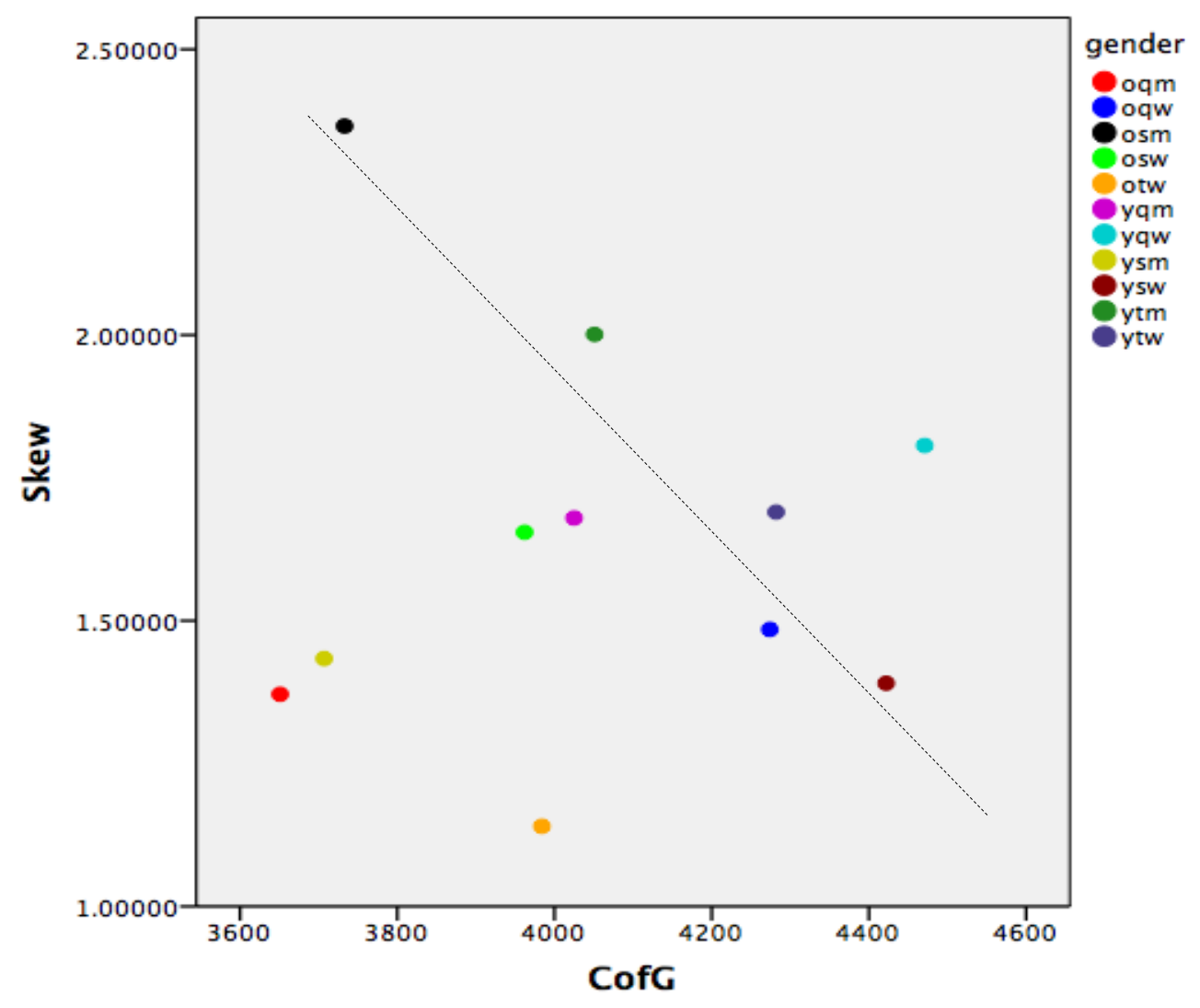

Figure 5.12: / $/$ CofG vs. Skewness

$/ \mathrm{J} /$, and it can only be fronted so far before it approximates $/ \theta / ; / \mathrm{J} /$ does not have these kinds of spatial restrictions, so gender marking through retraction is also a possibility, unconnected to secondary turbulence.

$/ \mathrm{J} /$ is voiceless, so we do not have to consider any effect of voicing. Ideally, a comparison would be possible with $/ 3 /$, but recall from $\$ 3.4 .2$ that there were too few tokens of $/ 3 /$ in this data to make even a cursory description reliable. We will focus instead on $/ \mathrm{J} /$ on its own, and with respect to $/ \mathrm{s} /$ and $/ \mathrm{z} /$ in place of articulation.

\subsection{1 / $/$ : : Distribution}

\subsubsection{Means}

The means for Centre of Gravity (CofG) and Skewness for $/ \mathrm{g} /$ are presented in Tables 5.17 and 5.18, respectively. The groups with the lowest CofG are men, and those with the highest are women, but the range is not as large as observed for either $/ \mathrm{s} /$ or $/ \mathrm{z} /$. This suggests that there may be a gender effect - if men tend to have $/ \mathrm{s} /$ and $/ \mathrm{z} /$ retracted relative to women, there is no particular reason to think that $/ \mathrm{J} /$ would not also be somewhat retracted - but it may not be a sufficiently strong effect to carry much social information. The standard deviations within the majority of the groups is also 
comparable, although younger trans men show a more loosely-packed distribution than the others.

With respect to Skewness, the gendered groups seem more disordered than they did for either $/ \mathrm{s} /$ or $/ \mathrm{z} /$ : there was a general reversal of the ordering between these two measures, so groups that had a high CofG tended to have a low Skewness. With this $/ \mathrm{J} /$ data, however, the picture is less clear; older trans women, for instance, have a very low Skewness, but only a mid-range CofG. It may be that Skewness, then, is more salient with articulations further forward than $/ \mathrm{J} /$, which could be interpreted as supporting the idea that Skewness may have something to do with the area of contact between the tongue and the roof of the mouth. As the tongue is retracted from an $/ \mathrm{s} /$ position and into $/ \mathrm{J} /$, the sides of the tongue may naturally make contact along a greater distance.

The scatterplot of CofG plotted against Skewness (Figure 5.12) shows that the relationship between these two measures is not as consistent as seen with either $/ \mathrm{s} /$ or /z/. While there are some similarities (it is true, for example, that older straight men are still relatively isolated, and that the cluster of older queer men, older trans women, and younger straight men is still in the lower left quadrant), the overall trend is not as clearly linear. This suggests that, for $/ \mathrm{J} /$, the expected correlation of Skewness and CofG is not strongly expressed, and may be a quality of further-fronted sibilants only.

Alternatively, this could be a consequence of the smaller range observed with CofG. With the same number of data points compressed into a smaller space, deviations from the predicted line may be proportionately larger than they would seem over a larger range. We could explore this relationship statistically, but since this scatterplot shows only raw means, uncorrected for effects of linguistic factors, it is not necessarily worth pursuing. The multivariate analysis will provide a better idea of the relationship between gendered identity and these spectral moments.

\subsubsection{Homogeneous subsets}

Tables 5.19 and 5.20 show the homogeneous subsets for Centre of Gravity and Skewness, derived from Tukey post-hoc following two ANOVAs (CofG: F(10, $1955)=21.028, \mathrm{p}<0.001$; Skew: $\mathrm{F}(10,1955)=25.453, \mathrm{p}<0.001)$.

These tables show a much more gradient relationship between gendered identity and both CofG and Skewness, as shown through the high degree of overlap between the homogeneous subsets. For CofG in particular, it is not possible to partition the gendered groups so that all of the groups in each subset is significantly different from all of the groups in the other subsets. With Skewness, older straight men constitute a group in their own right, but this is not entirely surprising, given the differences that we have seen between these men and the other groups with the other sibilants. 


\begin{tabular}{|c|c|c|c|c|c|c|c|}
\hline \multicolumn{8}{|c|}{ Table 5.19: / $/$ / Centre of Gravity: Homogeneous subsets (Tukey HSD ${ }^{\mathrm{a}, \mathrm{b}}$ ) } \\
\hline \multirow[b]{2}{*}{ Gender } & \multirow[b]{2}{*}{$N$} & \multicolumn{6}{|c|}{ Subset for alpha $=0.05$} \\
\hline & & 1 & 2 & 3 & 4 & 5 & 6 \\
\hline oqm & 158 & 3631 & & & & & \\
\hline ysm & 152 & 3755 & 3755 & & & & \\
\hline osm & 184 & 3770 & 3770 & 3770 & & & \\
\hline osw & 284 & & 3971 & 3971 & 3971 & & \\
\hline otw & 174 & & 3981 & 3981 & 3981 & & \\
\hline yqm & 200 & & & 4043 & 4043 & 4043 & \\
\hline ytm & 196 & & & & 4066 & 4066 & \\
\hline oqw & 155 & & & & 4219 & 4219 & 4219 \\
\hline ytw & 105 & & & & & 4305 & 4305 \\
\hline ysw & 199 & & & & & & 4449 \\
\hline yqw & 159 & & & & & & 4485 \\
\hline Sig. & & 0.875 & 0.235 & 0.057 & 0.127 & 0.082 & 0.072 \\
\hline
\end{tabular}

Means for groups in homogeneous subsets are displayed.

${ }^{a}$ Uses Harmonic Mean Sample Size $=169.223$.

${ }^{b}$ The group sizes are unequal. The harmonic mean of the group sizes is used. Type I error levels are not guaranteed.

\begin{tabular}{|c|c|c|c|c|c|}
\hline \multicolumn{6}{|c|}{ Table 5.20: / / / Skewness: Homogeneous subsets (Tukey HSD ${ }^{\mathrm{a}, \mathrm{b}}$ ) } \\
\hline \multirow[b]{2}{*}{ Gender } & \multirow[b]{2}{*}{$N$} & \multicolumn{4}{|c|}{ Subset for alpha $=0.05$} \\
\hline & & 1 & 2 & 3 & 4 \\
\hline osm & 184 & & & & 2.7971 \\
\hline ytm & 196 & & & 1.9954 & \\
\hline oqm & 158 & & & 1.8478 & \\
\hline yqw & 159 & & 1.7917 & 1.7917 & \\
\hline yqm & 200 & & 1.7121 & 1.7121 & \\
\hline osw & 284 & & 1.6768 & 1.6768 & \\
\hline ytw & 105 & & 1.6467 & 1.6467 & \\
\hline oqw & 155 & 1.4341 & 1.4341 & & \\
\hline ysm & 152 & 1.4338 & 1.4338 & & \\
\hline ysw & 199 & 1.3906 & 1.3906 & & \\
\hline otw & 174 & 1.1435 & & & \\
\hline Sig. & & 0.419 & 0.053 & 0.164 & 1 \\
\hline \multicolumn{6}{|c|}{ Means for groups in homogeneous subsets are displayed. } \\
\hline $\begin{array}{c}{ }^{a} \text { Uses } H c \\
{ }^{b} \text { The gro } \\
\text { used. Typ }\end{array}$ & onic & $\begin{array}{l}\text { n Sample } \\
\text { inequal. } T \\
\text { ls are not }\end{array}$ & $\begin{array}{l}=169.22 \\
\text { armonic } \\
\text { ranteed. }\end{array}$ & of the $g$ & sizes is \\
\hline
\end{tabular}




\begin{tabular}{ll}
\hline $\begin{array}{l}\text { Table 5.21: } \\
\text { analysis }\end{array}$ & \\
\hline Factors retained as significant & Sig. \\
\hline Position in word & $\mathrm{p}=0.004$ \\
Following segment: place \& manner & $\mathrm{p}=0.007$ \\
\hline no social factors retained & \\
\hline
\end{tabular}

\begin{tabular}{ll}
\hline $\begin{array}{l}\text { Table 5.22: Summary of } / \mathrm{J} / \text { Skewness multi- } \\
\text { variate analysis }\end{array}$ \\
\hline Factors retained as significant & Sig. \\
\hline Following segment: manner & $\mathrm{p}<0.001$ \\
Preceding segment: place \& manner & $\mathrm{p}=0.028$ \\
\hline no social factors retained & \\
\hline
\end{tabular}

\section{7 .2 / $/$ : Statistical modelling}

The best-fit multivariate analysis of $/ \mathrm{J} /$ for both Centre of Gravity (CofG) and Skewness are summarised in Tables 5.21 and 5.22, where we immediately note that none of the social factors were retained as significant in any model. Recall that these models included the eleven-way gendered identity categories, as well as the constituents (age, gender, identity) considered separately: none of these factors were retained in any of the models, meaning that the variation that we observed in the distributional discussion is best explained in terms of linguistic constraints.

For CofG, the linguistic factors retained as significant were the position in the word (initial, medial, or final), and the following segment. This is in contrast to our findings for the other sibilants, where the most relevant linguistic factors were the preceding segments. These differences may in part be due to the phonemic distribution of $/ \mathrm{f} /$ : many of the word-medial tokens are likely to have been in -tion and -ssion affixes, which could very well have distinct articulatory properties compared to $/ \mathrm{J} /$ in other morphological contexts (e.g., shin, wishes, finish). This is a more phonetic question than what we are interested in here, however, so we will leave this avenue of inquiry for future research.

For Skewness, the manner of articulation of the following segment was retained, as were both the manner and place for the preceding segment. /s/ and /z/ were also sensitive to the following place of articulation, and to a lesser extent, the preceding one. It is interesting that Skewness is not constrained by the position in the word, in spite of its relevance to CofG. Again, however, a fine-grained discussion of the morphological status of $/ \mathrm{J} /$ is beyond the scope of this report. 


\subsubsection{Discussion: / $\mathbf{f} /$ as a site for gendered identity}

The evidence presented in the previous sections demonstrates that $/ / /$ is not a site for gendered identity presentation in New Zealand English. This is broadly in line with previous work on fricatives in English (e.g., Fox \& Nissen 2005; Maniwa, Jongman \& Wade 2009), which generally agrees that gender differences are minimal with $/ \mathrm{J} /$ as compared to $/ \mathrm{s} /$.

However, our understanding of the gendered nature of sibilants more broadly would have been incomplete without including $/ \mathrm{J} /$, as it corroborates the hypothesis that the gendered indexicality of sibilants seems to be related to the frontness of articulation: the front-most $/ \mathrm{s} /$ has overt and accessible associations with gender, the somewhat retracted $/ \mathrm{z} /$ shows the same patterns but does not seem to carry /s/'s stereotyped associations, and the most retracted $/ \mathrm{J} /$ does not show any gendered effect under statistical analysis.

\subsection{General discussion of sibilants in New Zealand English}

The goal at the outset of this chapter was to explore the social dimensions of variation in sibilants in New Zealand English, to investigate the degree to which they can be used to signal identity. This was prompted partly by previous research into /s/ in particular, and my own observations of enormous differences in $/ \mathrm{s} /$ realisation between women presenting contrastive femininities. We focused on three sibilants, $/ \mathrm{s} /, / \mathrm{z} /$, and $/ \mathrm{J} /$, as these constitute two pairings of minimal phonemic difference $(/ \mathrm{s} /$ and $/ \mathrm{z} /$ differing in voicing but matched in place and manner of articulation, and $/ \mathrm{s} /$ and $/ \mathrm{g} /$ differing in place but matched in voicing and manner). They also occur often enough in the data to be examinable under the variationist lens.

What we have observed is that gendered identity is reflected in the spectral distributions of $/ \mathrm{s} /$ and $/ \mathrm{z} /$ (although not in as socially accessible a way with $/ \mathrm{z} /$ ), but it is completely absent in the $/ \mathrm{J} /$ data. There are a few reasons why we might see this kind of differentiation between the three sibilants. The generally low rate of occurrence for $/ \mathrm{J} /$ as compared to the other segments might simply make it a less socially useful investment in terms of presenting a gendered identity, or there may be limitations on the range of frequencies that will be interpreted as $/ / /$ by interlocutors - it is certainly true that there was a much smaller range of CofG values observed with $/ \delta /$ than there was with either $/ \mathrm{s} /$ or $/ \mathrm{z} /$. What is a more interesting question is why $/ \mathrm{z} /$ does not carry consciously interpretable social information, despite having a very similar distribution across the various gendered identities as $/ \mathrm{s} /$, and ostensibly the same place of articulation.

Over the course this chapter, we have developed the possibility that the accessibly gendered indexicality of sibilants is due to the secondary turbulence in the 


\begin{tabular}{lccc}
\hline \multicolumn{4}{c}{ Table 5.23: Gendered effects and meanings } \\
\multicolumn{2}{c}{ across sibilants by place of articulation } \\
\hline sibilant & $\begin{array}{c}\text { CofG range } \\
(\mathrm{Hz})\end{array}$ & $\begin{array}{c}\text { gendered } \\
\text { pattern }\end{array}$ & $\begin{array}{c}\text { gendered } \\
\text { stereotype }\end{array}$ \\
\hline$/ \mathrm{s} /$ & $5365-7553$ & yes & yes \\
$/ \mathrm{z} /$ & $4577-6915$ & yes & no \\
$/ \mathrm{g} /$ & $3631-4485$ & no & no \\
\hline
\end{tabular}

airflow, and therefore correlates with the frontness of articulation (see Table 5.23). If gendered indexicality flows from proximity to the front teeth (the source of secondary turbulence - e.g., Catford 1968: 322), then it follows that the most fronted realisations of $/ \mathrm{s} /$ should be the most gender-marked. It certainly seems true anecdotally that they are the most perceptually salient: an extremely fronted /s/ has a very high-pitched character, and can even begin to take on some of the qualities of whistling. From this perspective, high-CofG /s/ is fronted relative to some neutral, unmarked /s/, meaning that those who produce these fronted variants - a group dominated by younger women, as we saw in our discussion of $/ \mathrm{s} /$ and $/ \mathrm{z} /$ - are the ones constructively deviating from the unmarked category: it is not the case that /s/ is neutrally very fronted, and that retractors are doing something to set themselves apart.

We should briefly also make note of deviations of /s/ in the opposite direction, towards a more retracted, $/ \mathrm{J} /$-like articulation. Recall that in section $\$ 5.4 .2 .1$ it was noted that all tokens of (str) were excluded from analysis, because although there is a change in progress said to be occurring such that /st $\mathrm{I} /$ begins to approximate $[\mathrm{ft} \mathrm{I}]$ in words such as street, string, strong, etc., there was limited evidence of a gendered production effect in this data. This may be partly because the number of tokens for (str) was too low for a rigorous variationist analysis (with only around 30 tokens per gendered identity group), but it may also be that this innovation is not a marker of gendered identity in New Zealand English. However, it does carry overt social meaning in other varieties of English: see Appendix C for a list of blog posts (mainly reflecting the observations and interpretations of speakers of American varieties of English) that comment explicitly on the phenomenon.

What is interesting in these online discussion threads is the degree to which (str)-retraction is gendered and racialised. When black people are observed retracting (str), they are criticised for sounding uneducated and poor. When young women are observed retracting it, they are criticised in much the same way they always are: they are destroying the English language, they sound lazy, they are cringe-worthy and should learn to speak properly. When straight men are observed performing this same retraction, they are said to be enacting a hyper-masculine anti-lisp. In other words, when straight white men do it, it is socially acceptable: when anyone else does it, they are dysfunctional in some way. This study did not find any straightforward gendered differences in production in Auckland, and so the variable was dropped from the thesis. This does not rule out the possibility of gendered or ethnic differences in perception; 
this will need to be studied separately.

The relevance of this commentary on (str) retraction in non-NZ varieties of English is that both ends of the acoustic space for /s/ are open to commentary, which means both fronted and retracted variants can be marked. This suggests that there is a mid-range for /s/ that is socially neutral, and that deviations in either direction are potentially subject to commentary.

\subsubsection{A model of social meaning for sibilants}

In our discussion of /s/, we put proposed a dual-scale model for the interpretation of gendered performance, in which the same linguistic feature is interpreted differently depending on the perceived gender of the speaker. This intuitively fits with our observations on linguistic commentary in the previous section: men and women doing the same thing - retracting (str), for example, or indeed fronting $/ \mathrm{s} /-$ are perceived as doing different things. Our schema from section $\$ 5.5 .3$ (revised parallel continua) is reproduced here (Figure 5.13).

This model contrasts between different styles or types of masculinity and femininity, rather than assuming that there is a single continuum that runs from masculine to feminine. Each of these different styles is associated with a field of indexical values, and speakers are able to draw on these associations through their production of sibilants; or rather, through their production of $/ \mathrm{s} /$. We have seen that $/ \mathrm{f} /$ does not carry any gendered meaning or show any gendered pattern under statistical analysis, and although /z/ does show the same kind of pattern as /s/, it does not seem to be interpretable in the same way or the same degree. We speculated that this difference could be due to the relative retraction of $/ z /$, or to the effect of voicing. The similarities in the shape of the distributions for /s/ and /z/ likely reflect the organisation of the phonemic inventory, with /s/ and /z/ stored and processed as voiced and voiceless counterparts, but for whatever articulatory reason, /z/ is simply produced further back in the mouth, which would account for both the similar sets of constraints operating across the two segments (including the social factors) and the lack of accessible social associations (due to either voicing or place of articulation). So the model in Figure 5.13 can account for our observations with /s/, which in turn can be used to explain our observations with $/ z /$. It

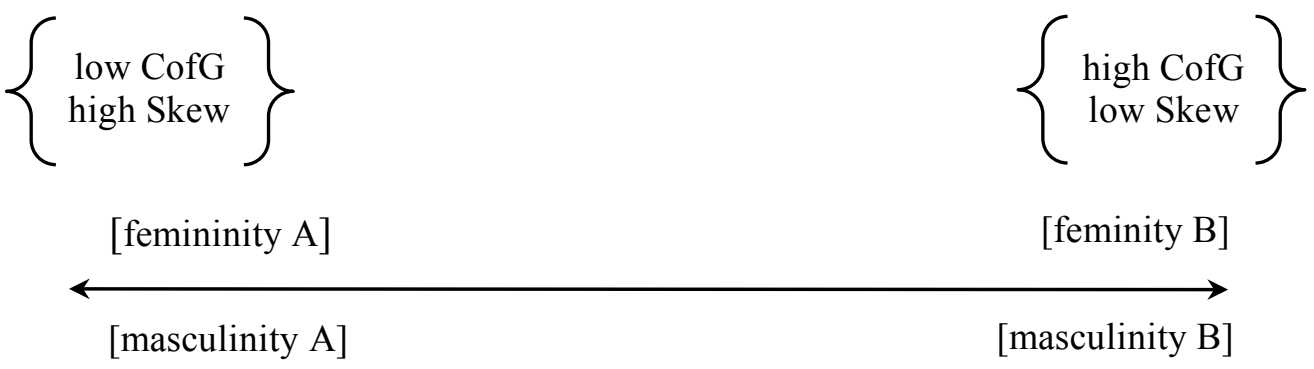

Figure 5.13: Revised parallel continua for gendered indexicality of /s/ 
is only $/ \mathrm{f} /$ that does not fit this pattern, but there is no particular reason why $/ \mathrm{f} /$ should index gendered identity, particularly if we are correct in our proposal that the indexicality is linked to the secondary source of turbulence.

The two groups most diametrically opposed to each other with respect to /s/ production are older straight men and younger women, and these two groups make an interesting social dyad. On the one hand, the group of older straight men in this corpus is quite strongly associated with traditional masculinity as well as the traditional social order: they are athletic and success-oriented in their respective fields, and in the interviews, they position themselves as the strong foundation on which their families are built, protectors of their wives and children. Although they have accepted the realities of the social changes that have been wrought in New Zealand since their childhoods (e.g., women pursuing higher education and professional lives), they have an internalised orientation to some of these more traditional gender roles, and an investment in their maintenance. Graeme and Jeff in particular are quite explicit in explaining their feeling that some social roles are more naturally suited to men, and others are more naturally suited to women, and they express a certain sense of nostalgia for the simplicity of these older systems. These are men who were raised in a particular social context that favoured men such as themselves, and they are in some ways resistant to the social disruptions that are unbalancing their privileged positions.

On the other hand, the groups of younger women - queer, straight, and trans position themselves as free actors in their own lives: they have pursued or are pursuing education in areas that interest them, they aspire to careers beyond marriage and childrearing, and they are critical (sometimes explicitly, particularly among queer and trans women) of these traditional gender norms that dictate what their expectations should be. Their investment is in carving out the life that they want for themselves, and they are willing to disrupt the gendered expectations of their parents' generation to do it.

These two groups have a natural antagonism, which maps onto the types of criticism that young women tend to be subjected to: they dress wrong, they speak wrong, they study the wrong things, they have the wrong ambitions. In essence, these criticisms are rooted in the idea that women are doing things differently than they did not so long ago, and these changes are beginning to de-privilege traditionally hegemonic power structures. In other words, young women are deviating from what older (straight) men think they should be doing, and these social tensions have become part of the respective identities of young woman and older straight man; this in turn means that they are available for linguistic encoding.

So perhaps our A and B types of masculinity and femininity in Figure 5.13 above correspond to traditional and contemporary models of gender. Older speakers who are more invested, or perhaps more entrenched, in these traditional models tend to produce /s/ towards one end of the CofG-Skewness continuum, while those who are more oriented to the contemporary tend to cluster towards the other end of this 


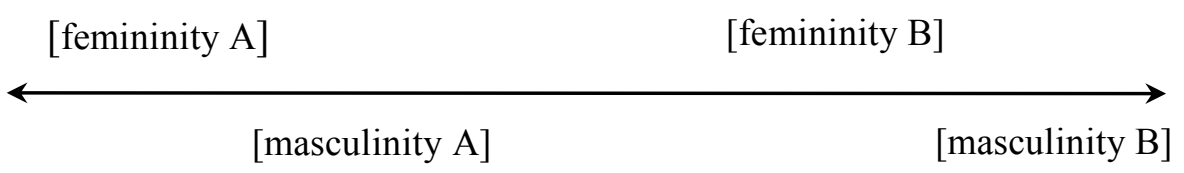

Figure 5.14: Schema of social interpretability of /s/

spectrum. The positioning of the other gendered groups with respect to these two poles aligns with their orientation to these two extremes.

So what do we make of the fact that the most B-type masculine productions of $/ \mathrm{s} /$ are not as fronted as the most B-type feminine productions? It could be that men are inherently less invested in the new social order: although queer men are distancing themselves from the heteronormative power structures that traditionally defined and devalued their identities, it does not mean that they are entirely committed to fully dismantling the patriarchal order. After all, they are still men, and they still benefit from the social asymmetries that privilege men over women. A more realistic schema, then, might offset the masculine and feminine poles with respect to each other, bringing them more closely into alignment with where our phonetic observations (especially for $/ \mathrm{s} /$ ) would place them, as in Figure 5.14.

Of course, we have not yet addressed the question of the indexical fields associated with these types of masculinities and femininities, we have only noted that a model such as that in Figure 5.1.4 more or less represents the distribution of $/ \mathrm{s} /$ in gendered space. We will develop and refine our notions of gendered indexicality in chapter seven. First, however, we will look at variation in the system of New Zealand English vowels. 


\section{Chapter six: Vowels}

\subsection{Introduction}

It is not immediately obvious why vowels should index gender in any meaningful way. After all, vowels convey so much linguistic information critical to identifying spoken words that it's not clear that there's much room left for gendered presentation. The difference between the word bit and the word bet, for example - or indeed, bat, but, boot, bought, bite, boat, etc. - can be characterised by relatively small articulatory and acoustic changes.

And yet we know that vowels do index some aspects of identity. Many of the differences between accents come down to vowels, for example. In New Zealand, people jokingly assert that Kiwis eat "fush and chups", while Australians order "feesh and cheeps". Although this is a clear exaggeration, it nevertheless highlights that people are aware of, and potentially able to assert control over, minor differences in vowel quality. Advertisers also make use of vowel manipulation, with a prominent chain of grocery stores producing ads that deliberately conflate the words "beer" and "bear", and "cheers" and "chairs", a fairly widespread merger in the vowel system of NZE. These particular vowels have crossed the threshold of salience for speakers of NZE, and are subject to overt commentary and joking; it is entirely possible that there are other vowels that index different aspects of identity, but which members of the speech community are not aware of.

Additionally, the vowel system in NZE is undergoing a number changes (see e.g., Gordon \& Maclagan 2001 for the NEAR-SQUARE merger; Maclagan \& Hay 2007 for the short front vowel shift; Marsden 2013 for adolescents and regional variation), and typically, variationist studies of sound change find that certain women tend to lead changes from below (e.g., Labov 1972) - that is, changes that people are not aware of. But which women? Early studies of phonetic variability used sex as a stand-in for gender. With a multi-faceted construction of gender, we may uncover more subtle patterns of variability. Indeed, studies of gay men in North America (e.g., Podesva 2011 for California, USA; Rendall, Vasey \& McKenzie 2008 for Alberta, Canada) have found that English vowels can index different masculinities, so there is no particular reason why the same cannot be true of New Zealand English.

Previous researchers have shown that some vowels are salient in New Zealand, some vowels can index sexuality in North American varieties of English, and that when a vowel system is undergoing change, there are likely to be gender differences. Because we cannot know in advance which vowels are likely sites for gendered identity work in NZE, we will begin by considering the vowel space as a whole, and identify likely variables from there before examining their distribution in more detail. 


\subsubsection{Roadmap for this chapter}

This chapter aims to investigate differences in vowel production between our different categories of gendered identity. Ten vowels have been selected as showing some kind of social trend in the data, and these are examined using multivariate analyses to tease relevant social factors out of linguistic ones, and the role of various social factors in this variation is considered. After these individual vowels are explored ( $\$ 6.3 .3$ to $\S 6.3 .5)$, variation in the vowel space as a whole is considered, using an overall rating of similarity and difference as another lens on gendered identity as encoded by vowels.

Before we look at the vowels directly, however, there are a number of methodological issues that need to be addressed. The data has already been automatically segmented and aligned (see $\$ 3.5 .1$ for a description of this process), but the vowels also will need to be automatically extracted, measured, and normalised to allow for comparison across speakers, and we must decide how we will conduct the analysis. These are discussed in the following sections, and are then followed by a description of the findings and a general discussion.

\subsection{Methods}

The broad methodologies used in preparing the interview sound files for analysis are discussed in chapter three, which details the transcription and alignment procedures. What follows in this section, then, is a description of how the vowels were extracted, measured, normalised, and analysed.

\subsubsection{Automatic vowel measurement}

As discussed in $\$ 3.5 .1$, the transcribed interview data was time-aligned using FAVE-align (Rosenfelder, Fruehwald, Evanini \& Yuan 2011), a forced alignment program developed by Penn Phonetics Laboratory at the University of Pennsylvania. This breaks down the word- or sentence-level transcription into a segmental one, and aligns these segments with the audio recording based on the spectral properties of the sound signal. The output from this alignment process feeds directly into FAVE-extract (Rosenfelder et al. 2011), which identifies and measures each vowel across two or three formants in the time-aligned transcript (depending on the quality of the recording, the third formant is not always automatically extractable), and returns a comprehensive data file for each recording.

An important decision that has to be made is at what point in the vowel we should take our measurements. The midpoint may seem the most intuitively obvious choice for monophthongs, but Evanini (2009) found that, in fact, the best point at which to take automated measurements is at a point one-third of the way through the vowel duration (p.65). He compared the values that were automatically measured at a number of 
points throughout the vowel's duration with those that were hand-measured by trained phoneticians, and found the lowest disagreement rates when the automatically-measured values were taken at the one-third point of the vowel (p.65). Indeed, he notes that in two corpora-based studies (Labov, Ash \& Boberg 2006; Hillenbrand, Getty, Clark \& Wheeler $1995)$ that relied on hand-measurement, vowels were generally measured at around the 30\% mark (Evanini 2009: 66-69), using the technique of looking for a steady state in the formants. Many of the improvements made to FAVE from the previous forced aligner at the Penn Phonetics Laboratory are based on Evanini's findings, so interested readers are directed there for a more detailed discussion of the back-end of the FAVE Programme Suite. The default point of measurement in FAVE is at one-third, with some minor adjustments (Rosenfelder et al. 2011a).

For monophthongs, then, we will use the FAVE preset. For diphthongs, there has been less rigorous analysis of measurement points compared to that which Evanini presents for diphthongs. Some researchers recommend measuring at $25 \%$ and $75 \%$ of the vowel's duration (e.g., van Bezooijen \& van Heuven 2010), while others (e.g., Wright \& Nichols 2014) recommend using $20 \%$ and $80 \%$. Another common approach (e.g., Watson \& Harrington 1999; also discussed in Evanini 2009) is to measure at the point where either F1 or F2 reached a peak (depending on the vowel in question); while this procedure does account for the different characteristics of each vowel, it is very difficult to automate, and is better-suited to studies using manual measurements. FAVE-extract automatically provides measurements at $20 \%$ and $80 \%$, so these are the measurement points used in this study.

\subsubsection{Output from FAVE-extract}

The output from FAVE-extract is organized primarily by vowel according to General American English pronunciation, but it also provides the source word itself, which is necessary for sorting and re-labeling vowels to more closely conform to New Zealand English pronunciation. By bulk-sorting the vowel output according to word first and then vowel, I was able to very easily re-assign American English vowels to more appropriate vowel sets. As a speaker of Canadian English, some New Zealand vowels were easier for me to categorize than others. New Zealand English has a few vowel distinctions that Canadian English does not, particularly in the low-back quadrant of the vowel space. Consequently, my coding of these vowels was checked and corrected by a linguistic colleague who is a native speaker of New Zealand English, and is familiar with Wells' (1982) lexical sets.

The advantage of using lexical sets over phones to identify vowels is that it makes it easier to compare between varieties. If we talk about the KIT vowel, we are talking about all of the words that have that same vowel sound in them - kit ship , this etc. - regardless of the actual pronunciation of that vowel. In New Zealand English, this vowel might be transcribed as [э], while in Canadian English it would be more likely to 
be transcribed as [I]; Australian English might use [I] to reflect its fronted realisation. If we were to compare these three varieties and only use the appropriate IPA symbol, it might be clear that in Canadian and Australian English we are talking about the same vowel, but without a solid foundation in the phonology of New Zealand English, it would not be obvious that the New Zealand vowel was the same phoneme. On the other hand, if we talk about the KIT vowel in each variety, then we are able to make unambiguous observations that KIT is retracted further back in NZE than it is in either Canadian or Australian English.

The lexical sets considered in this study are those adapted by Hay, Maclagan \& Gordon (2008) from Wells (1982), and are listed below along with examples of words from that set.

monophthongs (Hay, Maclagan \& Gordon 2008: 22)

FLEECE: seat, free, fiend, key

KIT: sit, Sydney, pretty

DRESS: set, head, Geoff, many

TRAP: sat, bad, gas, happy

START: cart, grass, dance, bath

STRUT: cut, butter, rough, money

LOT: cot, body, what

THOUGHT: cord, caught, call, draw

GOOSE: suit, boot, shoot, chute

FOOT: put, book

NURSE: shirt, work, hurt, fern

diphthongs (Hay, Maclagan \& Gordon 2008: 26)

MOUTH: hour, house, bout

CHOICE: boy, foil

PRICE: bite, fly, time

GOAT: boat, loam, go, close

FACE: play, frame, stain, faze

SQUARE: cared, aware, flares

NEAR: fears, cheered, here

One disadvantage of using lexical sets is that the distinction between phonemes and allophones is not always apparent. This is particularly true of NZE vowels with a coda/1/, which may be fully vocalised and thus have an enormous impact on the formants of the preceding vowel. At what point does this stop being a phonologically conditioned alternation and become its own lexical set? It has been suggested (Bauer \& Warren 2004a) that a GOLD lexical set be added to the list of NZE vowels, consisting of the GOAT vowel with a coda-/1/ (and include words such as whole, soul, fold, etc.). But what 
of other vowels with a coda-/1/, such as boil, pull, pool, curl, Sal and well? A vocalised /1/ exerts a strong effect on these vowels as well, so it is not clear why in principle these should not also have their own lexical sets. This discussion is beyond the scope of this project, however. But because of this known effect of $/ 1 /$, we have excluded all tokens with a coda-/1/ from the analysis.

New Zealand English is also a (largely) non-rhotic variety, leading to coda-/r/ vowels being either assigned to their own lexical set (e.g., NEAR and SQUARE), or classed with non-coda-/r/ vowels that have similar qualities and historical links (e.g., the START lexical set above). By and large, I have followed the conventions of phonetics and phonology in NZE; one exception, however, is the lexical set THOUGHT, which includes words like sort, cord, and north. As a non-speaker of NZE, my impression from listening to casual conversations of native NZE speakers is that these $/ r$-coda vowels do not always have the same qualities as the other words in the THOUGHT lexical set. Because of this perceived variability, I decided to code NORTH as a separate lexical set from THOUGHT, to investigate my intuitions.

Another distinction I initially made in assigning the measured vowels to lexical sets was to code KIT vowels with a coda-/y/ (e.g., wing, English, finger) separately, because they likewise did not sound consistently KIT-like to me. This THING lexical set showed so much inter- and intra-speaker variability that it was subsequently excluded from the analysis, but would be well worth investigating in the future.

There are two other lexical sets generally used when discussing NZE: commA and TOUR. The first of these refers to the unstressed vowel that is often transcribed as /o/ (schwa) in other varieties of English, and as /9/ in NZE. This was excluded because the acoustic analysis is only concerned with vowels that carry, at a minimum, secondary lexical stress (and are therefore not fully reduced). The second of these, TOUR (e.g., sure, fury, cure), is a problematic lexical set in that it has a lot of variety (sometimes pronounced with the NORTH vowel, other times with GOOSE; sometimes as a monophthong and sometimes as a diphthong; sometimes with a palatal on-glide and sometimes without). Additionally, it is not among the more frequent vowels in English, so it is difficult to get enough tokens through casual speech to be able to fully investigate this variability. Consequently, TOUR was excluded from this study.

\subsubsection{Outliers}

Because I extracted and measured the vowels automatically, there is a chance that mis-measurements have been included in the data. Short of visually inspecting every vowel token measured - which would essentially negate any time advantages of using automated processes in the first place - the easiest way to mass-exclude likely mismeasurements is to identify the outliers. There are a number of ways to identify outliers, many of which are statistically more complex than is necessary for this type of study. The two simplest methods involve measures of central tendency: namely, the mean and 
standard deviation (SD); and the interquartile range (IQR).

The first of these methods calculates the arithmetic mean (commonly referred as the 'average') of the set of measurements, and then calculates the standard deviation (SD) around this mean, assuming a normal distribution. One common approach to using SDs for identifying outliers is to consider anything that falls outside of $2 \mathrm{SD}$ from the mean as an outlier. This method retains the central $95.4 \%$ of the data, and excludes the highest $2.3 \%$ and the lowest $2.3 \%$. The disadvantage of this method is that the value of the arithmetic mean can be distorted by a small number of very high or very low measurements.

The second of these methods sorts the measurements numerically from smallest to largest, and identifies the range in which the central $50 \%$ of the measurements occur; this is the IQR. Measurements that are more than 1.5 IQR above or below this range (i.e., the lower end of the range minus 1.5 IQR, and the higher end of the range plus 1.5 IQR) are considered outliers. The disadvantage of this method is that, if the data is skewed away from a strictly normal distribution (if, for example, the arithmetic mean does not fall in the middle of the IQR), some measurements will likely be mis-identified as being outliers. However, the IQR is not strongly affected by a small number of extreme values, meaning that this approach is less susceptible to influence by the outliers that it is intended to identify. This is the method I adopted for this study.

For each individual speaker, then, I identified and excluded outliers for each vowel (classified according to lexical sets, as discussed in §6.2.2) based on measurements for both F1 and F2. For monophthongs, I used the default measurement point (discussed in section §6.2.1), and for diphthongs, I used both the $20 \%$ and $80 \%$ points. In total, less than 500 out of more than 36000 tokens were identified as outliers and excluded, meaning that the majority of the inter- and intra-speaker variability was retained.

\subsubsection{Vowel normalisation}

Due to physiological differences between speakers, not all realisations of a vowel are going to be directly comparable. Research has found that generally, women have a higher fundamental frequency (F0) than men, and this mean difference is attributed to differences in vocal tract anatomy in post-pubescent adults (e.g., Fant 1960; Whiteside 1996). These different F0s also entail systematic differences in the formants (F1, F2, F3, etc.) that are used in measuring vowels acoustically. To compensate for these physical differences between speakers, and thus to isolate the variation that is due to social factors from those that are due to biology, a number of vowel normalisation techniques have been developed by phoneticians. Each has its own strengths and weaknesses, and in many cases, are optimised for a particular type of data. Comprehensive reviews of some of these methods can be found in Hindle (1978), Disner (1980), Miller (1989), and Adank, Smits \& van Hout (2004), and are summarised in Thomas \& Kendall (2015a).

Vowel normalisation techniques can be developed using vowel-intrinsic (where 
each vowel token is normalised using information contained within that one token, e.g., F0, formants, etc.) or vowel-extrinsic (where the information used to normalise vowels is pooled from different tokens from the same speaker) techniques. Similarly, they can be speaker-intrinsic (where the normalisation procedure uses tokens from only speaker) or speaker-extrinsic (where normalisation draws on data from several speakers) (Thomas \& Kendall 2015a). Which of these methods is used depends on the type of data and the research question being investigated; for the purposes of this project, the two most promising candidates are Nearey (1977) and Lobanov (1971).

The differences between these two methods are not enormous (e.g., Thomas \& Kendall 2015b). Both are inherently vowel-extrinsic and speaker-intrinsic (they make use of multiple vowel tokens for each speaker), but both have been modified since their original inception so that they can be adjusted to accommodate different types of data. Because they are vowel-extrinsic, they do best when the whole vowel space is represented in the data, which is the case with this study. Neither is particularly good at comparing between dialects or languages with different vowel systems when the two lects are normalised together - but this project is concerned with only one variety of one language. Both generate outputs that are not in a transparent unit, and so must be scaled to produce results in Hertz. Disner (1980) found that Nearey is slightly better at reducing scatter, while Adank et al. (2004) found that Lobanov performed slightly better at a vowel discriminant analysis of Dutch.

As neither Nearey nor Lobanov is markedly better-suited for the data in this project, I chose to use Lobanov for the sake of expediency, as a Lobanov-normalised output is included as part of the FAVE Program Suite's options. FAVE-extract "follows the Lobanov (1971) normalization procedure, which transforms a speaker's vowel space into $\mathrm{z}$ scores using the overall grand mean and standard deviation for the whole vowel system" (Rosenfelder et al. 2011b). The formula used for this transformation is given in (1):

$$
F_{i}^{N}=\frac{\left(F_{i}-M_{i}\right)}{\delta_{i}} \quad(\text { Lobanov 1971: 606) }
$$

where $F_{i}^{N}$ is the normalised value for a particular formant of a particular vowel, $F_{i}$ is the non-normalised value, $M_{i}$ is the mean for that formant for all vowels (calculated individually for each speaker), and $\delta_{i}$ is the root mean square deviation about $F_{i}$.

The values used to scale the normalised results are given in (2):

$\begin{array}{lll} & \text { mean } & \text { st.dev } \\ \text { F1 } & 650 \mathrm{~Hz} & 150 \mathrm{~Hz} \\ \text { F2 } & 1700 \mathrm{~Hz} & 420 \mathrm{~Hz}\end{array}$

(Rosenfelder et al. 2011c) 


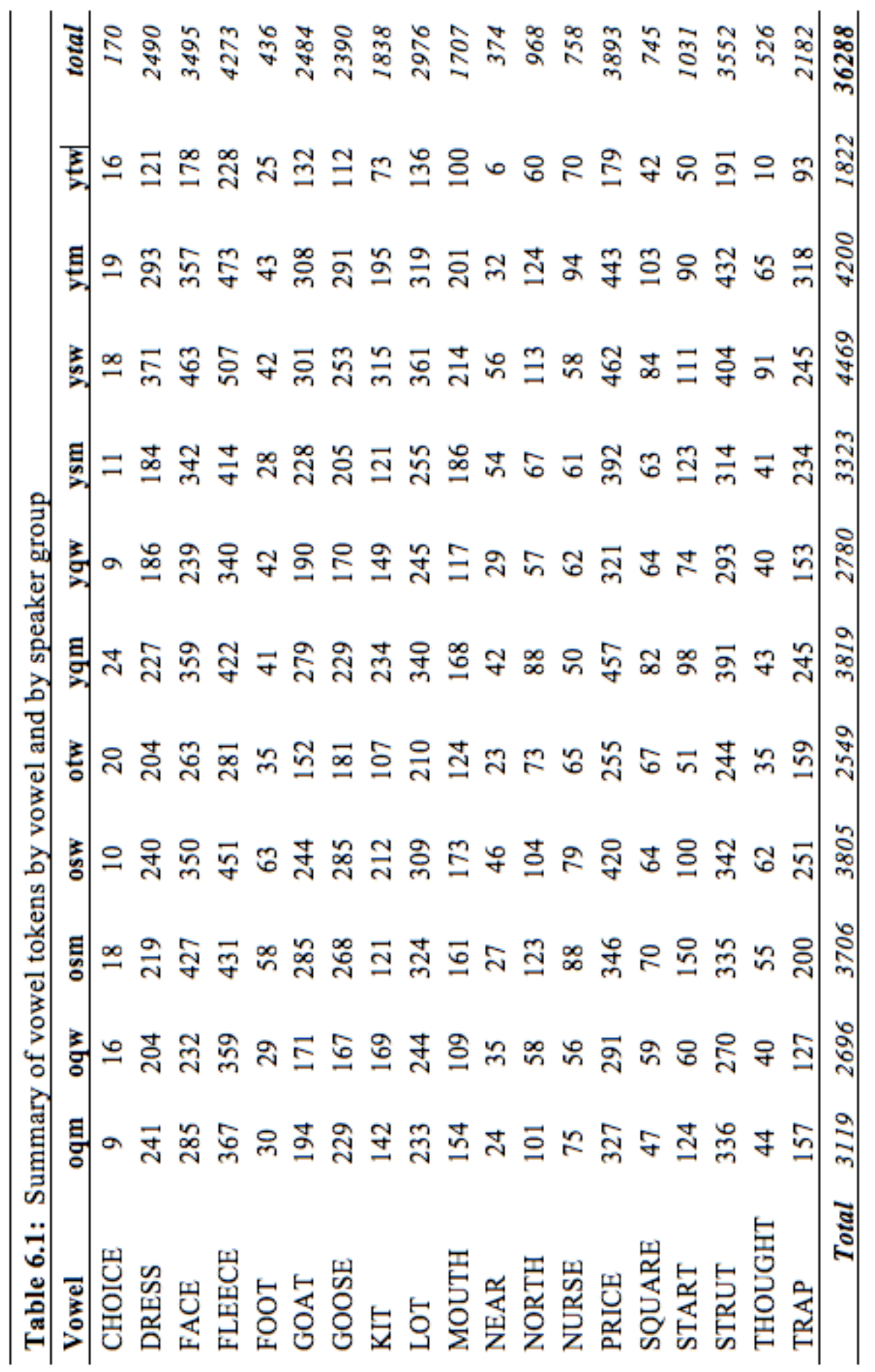




\subsubsection{Total number of vowel tokens}

On average, each speaker's interview extract yielded 700 to 1000 tokens of stressed vowels (defined as having primary or secondary lexical stress). In order to minimize the effect of very high-frequency words such as like, a maximum of 20 tokens of any given type (word) was retained for each speaker. The total number of vowel tokens retained for the analysis (after exclusions - see §6.2.3) was 36288 ; this is summarised in Table 6.1, broken down by vowel and by gender group.

Because the data is drawn from naturally-occurring spontaneous speech, the particular lexical items varied widely from speaker to speaker, depending on the narratives they were relating. So although some speakers produced more DRESS vowels than others, for example, the pooling of data across gender categories, and the generally high number of tokens overall, ensured that the data was not enormously skewed.

It should also be noted that for some vowels - most notably for CHOICE and NEAR - there were very few tokens even with the individual speakers pooled. This puts limitations on the types of analysis we can subject these particular vowels to, as tests for statistical significance between means (for example) are not reliable with such low Ns. Nevertheless, there is ample data to work with overall.

\subsubsection{What to compare: The Cartesian vowel plot}

It is standard practice in the phonetics of vowels to consider vowel dispersion on a plane, with the first formant (F1) constituting the vertical axis, and the second formant (F2) constituting the horizontal. This approach has several advantages. First, vowels can generally be differentiated based solely on these two measurements (higher formants can certainly provide useful articulatory detail on vowel production, but they are not strictly necessary to identify a given vowel), which means that a two-dimensional representation can adequately describe the vowel space of a particular language. Second, F1 is correlated inversely with vowel height (i.e., a high $\mathrm{F} 1$ value means a relatively low tongue position, and a low F1 value means a relatively high tongue position), while F2 is correlated with frontness or backness in the mouth (i.e., a high F2 value is articulated towards the front of the mouth, and a low F2 value is articulated towards the back), and in conjunction with $\mathrm{F} 3$ - with rounding. This means that by reordering the scales on the axes, the Cartesian vowel plot can be made to approximate the IPA canonical vowel diagram (see Appendix A), which in turn makes the association of the acoustic signal and the phoneme an intuitive one. Third, these principles apply regardless of the unit of measurement - a vowel plot constructed in this way provides the same information whether it is measured in Hertz, Mel, Bark, or using some other acoustic scale.

One major disadvantage of the Cartesian vowel plot, however, is that it lends itself primarily to analysis along one of the two axes. That is it say, it is straightforward to describe changes or differences along either the F1 or F2 dimension: vowel $X$ is higher than vowel $\mathrm{Y}$, or vowel $\mathrm{Z}$ is further forward than both. The articulatory reality of vowels, 
unfortunately, is such that many vowel changes over time occur at an angle relative to these axes, raising the question of the best approach to analysing such vowel trajectories. Certainly, they can be decomposed into their F1 and F2 components, and each component analysed independently, but this approach is unsatisfying, since changes in one may not in fact be independent of changes in the other. The Cartesian vowel plot makes these relationships easy to see, but it is not always obvious whether observed differences are statistically significant. A full statistical analysis of F1 and F2 simultaneously is quite complicated, particularly if one is interested in the linguistic factors - such as phonetic environment - that may be influencing the observed changes. The statistical approaches used by sociolinguists to analyse variation are not enormously well-suited for analysing two related, multidimensional dependent variables at the same time. What is needed, then, is a way to turn these two variables into one.

\subsubsection{Euclidean Distance}

One such approach is to calculate the Euclidean Distance (ED) between vowels and a fixed point in the vowel plot. This analysis was developed by Watson, Maclagan, King, Harlow \& Keegan (2016), who were looking at diachronic changes in the vowel system of te reo Māori, the language of the indigenous people of New Zealand. They observed that the incremental changes over generations for a particular vowel, when plotted on a Cartesian plane, fell along a straight line between two fixed vowels. By essentially redefining the relevant axis as the line between the two fixed vowels and then projecting vowel measurements onto that line, they were able to analyse the change as movement along one dimension - in other words, they had reduced their analysis to one dependent variable.

An important difference between Watson et al.'s (2016) ED calculation and the one used in this study is that, rather than defining a new axis and projecting the vowels onto this axis, I have instead analysed the vowel's distance out from a single reference point. Mathematically, the approach is quite simple, using nothing more complex than high school Euclidean geometry. Any two points on a plane can be joined by a line, and that line can be decomposed into two perpendicular vectors. In this case, those perpendicular vectors are the differences between the F1 (vertical) and F2 (horizontal) measurements for the two points, which are a fixed vowel and a vowel of interest (see Figure 6.1). We have now constructed a right-angle triangle, and we can use the Pythagorean Theorem to calculate the length of the hypotenuse, which is the Euclidean Distance that we are interested in. We can calculate a Euclidean Distance for each of the vowels of interest, using the same fixed vowel as a reference point, and we will have a series of measurements that we can use in our statistical analysis.

In order for this Euclidean distance to be meaningful, two conditions must be met. First, the various vowels of interest must fall in a fairly straight line. It is almost inevitable that they will not perfectly align, but there must be a clear trend towards a linear relationship. Second, this straight line must point towards a stable (or unchanging) 


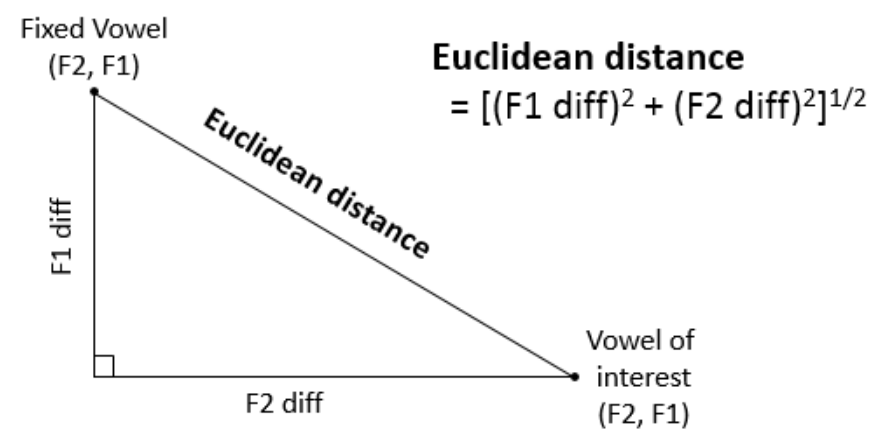

Figure 6.1: Calculating Euclidean Distance (ED)

vowel that can be used as a reference point. It is important that this reference point is on approximately the same line as described by the vowels of interest, because the Euclidean distance is a measurement of the relative distance of a given vowel to its reference point. If the reference point is not on this line, then the Euclidean distance measures will not accurately order the vowels of interest along the new axis (reference line). These conditions are illustrated in Figure 6.2, where A is the only case in which Euclidean distance would be an appropriate tool for analysis.

The magnitude of the ED that is calculated for each vowel analysed using this metric gives an indication of distance from the reference vowel, and does not correlate directly with vowel height or frontness. Rather, it must be interpreted in relation to the reference point - so for example, if the reference vowel is positioned higher in the vowel space than the target vowel, then a smaller ED will indicate a higher target vowel and a larger ED will indicate a lower vowel. If the reference vowel is lower than the target vowel, then a small ED will indicate a lower target and a larger ED will indicate a higher vowel. The reference vowel is stated explicitly for each vowel, and the interpretation of the magnitude is also made clear in each instance.

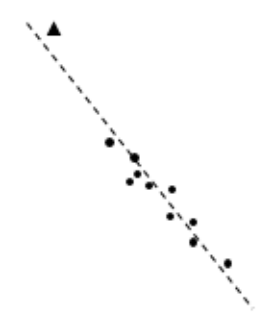

A

Vowels of interest (circles) lie in a straight line pointing towards the reference vowel (triangle).

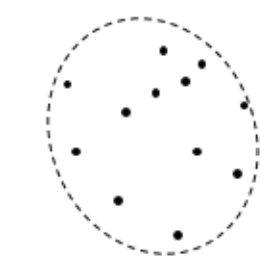

B

Vowels of interest do not lie in a straight line.

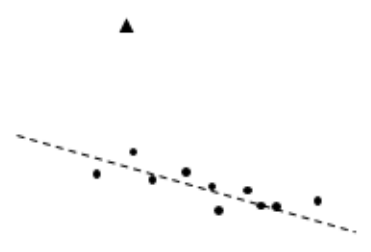

C

Vowels of interest lie in a straight line, but this line does not point towards the reference vowel.

Figure 6.2: Conditions for Euclidean Distance measurement 


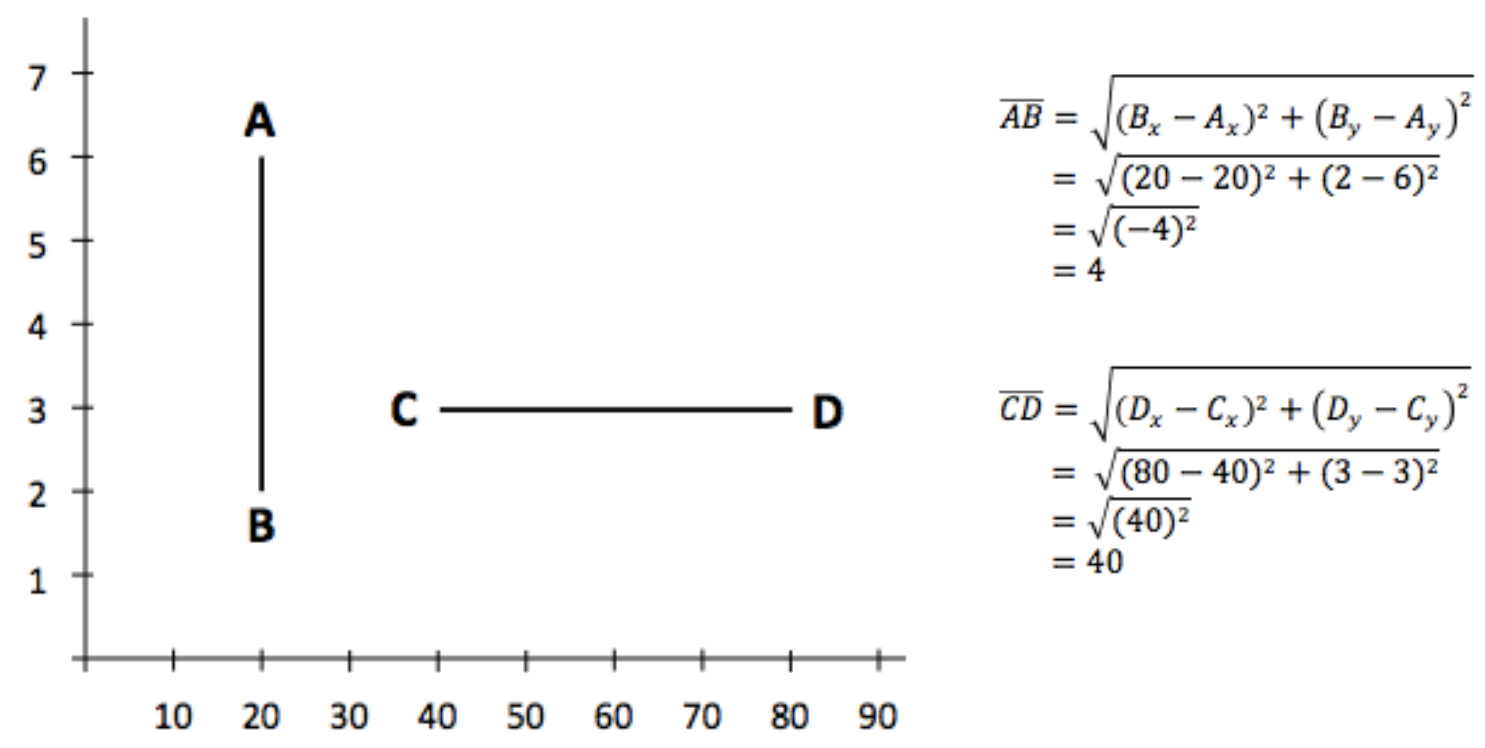

Figure 6.3: Exaggerated effect of different scales on $\mathrm{x}$ and $\mathrm{y}$ axes

One other point bears mentioning with respect to the Euclidean Distance metric as it is used in this study. Because the scales of F1 and F2 in vowel plots are not square that is to say, the range on the F1 axis is considerably smaller than the range on the F2 axis - the calculation could be seen to disproportionately favour differences in F2 over differences in F1 (see Figure 6.3). While it is true that, mathematically, the F2 range will always have a larger effect on the ED than the F1, there are two reasons why this is not actually a problem for this analysis. First, each ED calculation is presented in combination with a vowel plot, so that the ED value can be put into perspective. And second, the ED values are not used to compare different vowels, but rather different realisations of the same vowel. Since ED is only used when the vowels lie in a fairly straight line, two instances of the same vowel can be compared with a high degree of confidence.

\subsubsection{Scale}

The fact that the scales on two axes are not of the same magnitude ( $\mathrm{F} 1$ ranges from around $400 \mathrm{~Hz}$ to $900 \mathrm{~Hz}$; F2 from around $700 \mathrm{~Hz}$ to $2500 \mathrm{~Hz}$ ) means that differences in F1 and F2 are not going to be directly comparable - that is to say, a range of $100 \mathrm{~Hz}$ on the $\mathrm{F} 1$ axis is relatively larger than a $100 \mathrm{~Hz}$ range on the F2 axis. This mismatch is perhaps to be expected, however. If the first formant is associated with the tongue height during articulation, and the second formant with the tongue's front-back position, then it makes sense that the range would be greater for F2, since the oral cavity is longer than it is tall. Additionally, the relative positions of the vowels on these Cartesian plots approximates the IPA vowel chart in terms of relative positions, suggesting that the distances needed to discriminate phonemes are different with respect to F1 and F2. Thus, 
even though the differences are of a different magnitude, we actually can use the visual positions as an indicator of whether differences are phonemically meaningful.

\subsubsection{A note on the use of Hertz}

It is worth noting at this point that there is no clear consensus on how big a difference in $\mathrm{Hz}$ needs to be for it to be meaningful. Research conducted on just noticeable differences (JNDs) has shown that the human auditory system can detect modulations at frequencies of around $4 \mathrm{~Hz}$ (Zwicker \& Fastl 1990: 163), but this is not a particularly useful approximation of the acoustic signals comprising speech, particularly in light of Green's (1976: 260) observation that JNDs are sensitive to changes in intensity (loudness), and naturally-occurring speech is clearly not uniform in its intensity. Plomp (1976; 2002) discusses the human perception of complex harmonic signals in terms of octaves, noting that "there is a close relation between spectral differences for vowels measured in $1 / 3$ octaves and perceptual differences as judged by listeners" (2002: 22). The physiological and neurological properties of the human auditory system are such that researchers interested in perception have all but abandoned the use of Hertz, favouring instead a more psychoacoustically-informed measurement, such as the Mel scale (Stevens, Volkmann \& Newman 1937) or the Bark scale (Zwicker 1961).

This project continues to use Hertz for three reasons. First, many of the phonetics studies of New Zealand English have reported their findings in Hertz, so for comparative purposes, it makes sense to use the same scale. Second, although the automatic extraction program used to measure the vowels in this project (FAVE - see $\S 3.5 .1$ and $\S 6.2$.1 for a more detailed discussion of the program) does give Bark scale equivalents for many of the measurements it makes, it does not do this for offglides, meaning Bark is not particularly well-suited to the study of diphthongs. The conversion from Hertz to Bark requires the third formant (F3) to be measured as well, which is likewise not part of the FAVE output for offglides. The third reason for using Hertz is that this is a study of production more than it is one of perception, and the acoustic signal itself - independent of the hearer - can most meaningfully be described in terms of frequency.

\subsubsection{Analysis of gendered production}

There are two statistical approaches taken in this project to examine the gendered production of vowels: a post-hoc Tukey test for homogenous subsets following an ANOVA, and a multivariate analysis that models the constraints on variation (see \$3.6). As discussed previously, the Tukey HSD (\$3.6.1.2) tests for significance using an adjusted significance level, and the multivariate approach (Rbrul) favoured by variationist sociolinguists ( $\$ 3.6 .2)$ tests the relative effects of multiple parameters simultaneously. The disadvantage of this multivariate analysis is that it is not possible in Rbrul to identify significant differences within a factor group. The retention in the model of a factor group tells us that the factor group as a whole is a significant predictor of variation, but it does 
not tell us which factors within that group are significantly different from each other. Thus, for example, if one group of speakers has an effect of raising the mean frequency by $150 \mathrm{~Hz}$ (indicated by the coefficient provided by Rbrul), and another group raises by $250 \mathrm{~Hz}$, there is no statistical test to determine if that $100 \mathrm{~Hz}$ difference is significant. In that sense, it is like an ANOVA (in that it tell us that there is significance somewhere), but it does not come with post-hoc tests to more closely examine the factors within the group.

For the discussions that follow, then, the patterns for each vowel will be discussed with respect to the multivariate analysis: once other linguistic factors have been accounted for, how does gendered identity affect production? In developing the model for each vowel, both the preceding and following segments were considered. These factors were included to ensure that we weren't over-estimating the social factors, rather that being in and of themselves a focus of the analysis. Note that duration is not reliably measured when the transcription has been force-aligned, so this factor was not included in the analysis. As noted previously, all of the vowel tokens carried either primary or secondary stress, so stress was also not included in the model. The overwhelming majority of tokens carried primary rather than secondary stress, and when a small sample of tokens was tested, it was found that the exclusion of the secondary stress tokens did not significantly affect the observed means, so the primary and secondary stressed tokens were retained as one group.

With regards to the social factors which are the focus of this project, models were run using the composite gendered identity classification (age $\mathrm{x}$ identity $\mathrm{x}$ gender), and also using each of these social factors independently. The models developed using the two social configurations were compared using a Chi-square test in Rbrul, and the model with the better fit - that is to say, the model that best accounted for the observed variation - was taken as the basis for the ensuing discussion.

In $§ 6.3 .6$, when we shift the unit of analysis from individual vowels to a crossidentity comparison of the vowel space as a whole, we will make use of the Tukey Homogeneous Subsets rather than the multivariate analyses. Generally speaking, the differences between the ordering of the Rbrul output and the Tukey groupings are not enormous; where there are substantive re-rankings, these are usually within homogeneous subgroups, and there are only a handful of instances where this has an effect on the subgroupings themselves. Given that this second analysis is interested in a combined effect of multiple vowels simultaneously, the simplicity of using the Tukey HSD test outweighs the cost of any small discrepancies. The methodology of this vowel-spacecomparison is discussed in detail in $§ 6.3 .6$. 


\subsection{Findings}

Now that we have decided on an approach to analyse the vowel tokens we have collected, we can turn our attention to the vowel measurements themselves. This section will begin with an overview of the vowel space of New Zealand English as a whole, before moving on to consider individual vowels in closer detail. A gendered breakdown of the means for each vowel is provided in Appendix D.

\subsubsection{NZE vowel space generally}

The overall shape of the vowel space of NZE in Auckland is shown in Figure 6.4, which includes mean F1 and F2 values averaged across all speakers, regardless of gendered identity. It is broadly triangular, anchored at the three vertices by FLEECE (high front), NORTH (high back), and START (low). TRAP protrudes slightly forward of the line between FLEECE and START, but not to the extent that we would characterise the vowel space as a quadrilateral.

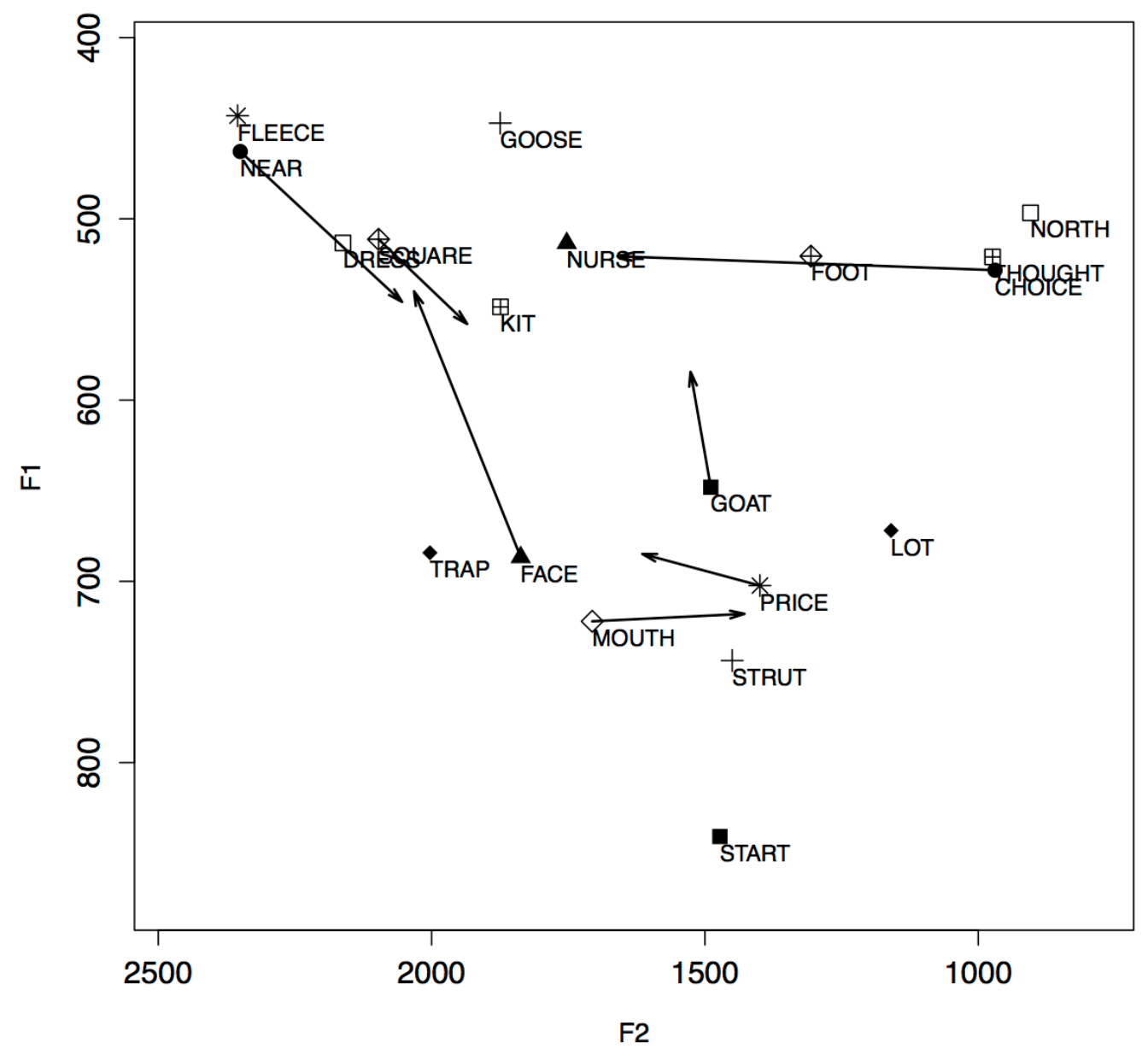

Figure 6.4: Overall mean values for all vowels 
This is broadly in line with previous accounts of the geometry of NZE vowels. Working with data from Canterbury collected in the early 1980s from speakers in their 20s, Gordon, Campbell, Hay, Maclagan, Sudbury \& Trudgill (2004: 25-27) produced vowel plots for both men and women speakers of NZE. Although this means that we cannot make direct comparisons of frequencies between the two data sets, we can make some general observations about the vowel space as a whole.

There are three prominent differences between the two data sets. First, GOOSE is much further forward in the Auckland data than in the Canterbury data. In particular, it is further forward than NURSE, which is a direct reversal of Gordon et al.'s findings. It is also higher than NURSE, although it is difficult to say whether this is because GOOSE is higher or NURSE is lower. Hay, Maclagan \& Gordon (2008) also have GOOSE occurring farther back than NURSE, although the F1 (height) difference that they report is comparable to that seen here. Second, there is a marked difference in height between START and STRUT in this Auckland data, where Gordon et al. found the two nearly merged; indeed, this near-merged realisation is reported elsewhere (e.g., Warren 2006a; Watson, Harrington \& Evans 1998). Warren observed that the principal distinction between the two lexical sets was one of quantity (vowel length) rather than quality (formant frequencies), although he did note that younger speakers may be starting to separate them again.

The third main difference between the two data sets is the status of NEAR and SQUARE. Gordon et al. report that the onset for these two diphthongs is nearly merged, particularly for men (2004: 30-31). This is also what Hay, Maclagan \& Gordon (2008) report, noting that "for most younger speakers of NZE, there is no longer any distinction between word pairs like ear and air or cheer and chair or beer and bear" (p.27). The merging (or near-merging) of NEAR and SQUARE in NZE has been explored at great length by many researchers, and is sometimes discussed as a fait accompli. Nevertheless, this data suggests that the two are less fully merged than some previous studies have found. Possible sources for differences between these findings and those of previous studies include the period over which the data was collected (the ATC having been collected in 2013-2015, making it considerably more recent than many previous corpora), the region(s) in which the data was collected (ATC focusing on the Auckland area, while other prominent NZE corpora are from Canterbury and Wellington), and the type of data (the ATC uses spontaneous, conversational data, while many previous studies have looked at read speech and word list data, or have been from a perceptual perspective rather than a production one). See $\S 6.3 .5 .1$ for a more detailed exploration of NEAR and SQUARE in this data.

\subsubsection{A closer look at the vowel space}

Looking at the overall vowel space of NZE with all speaker distinctions collapsed is overly simplistic, from both a linguistic and a social point of view. Figure 


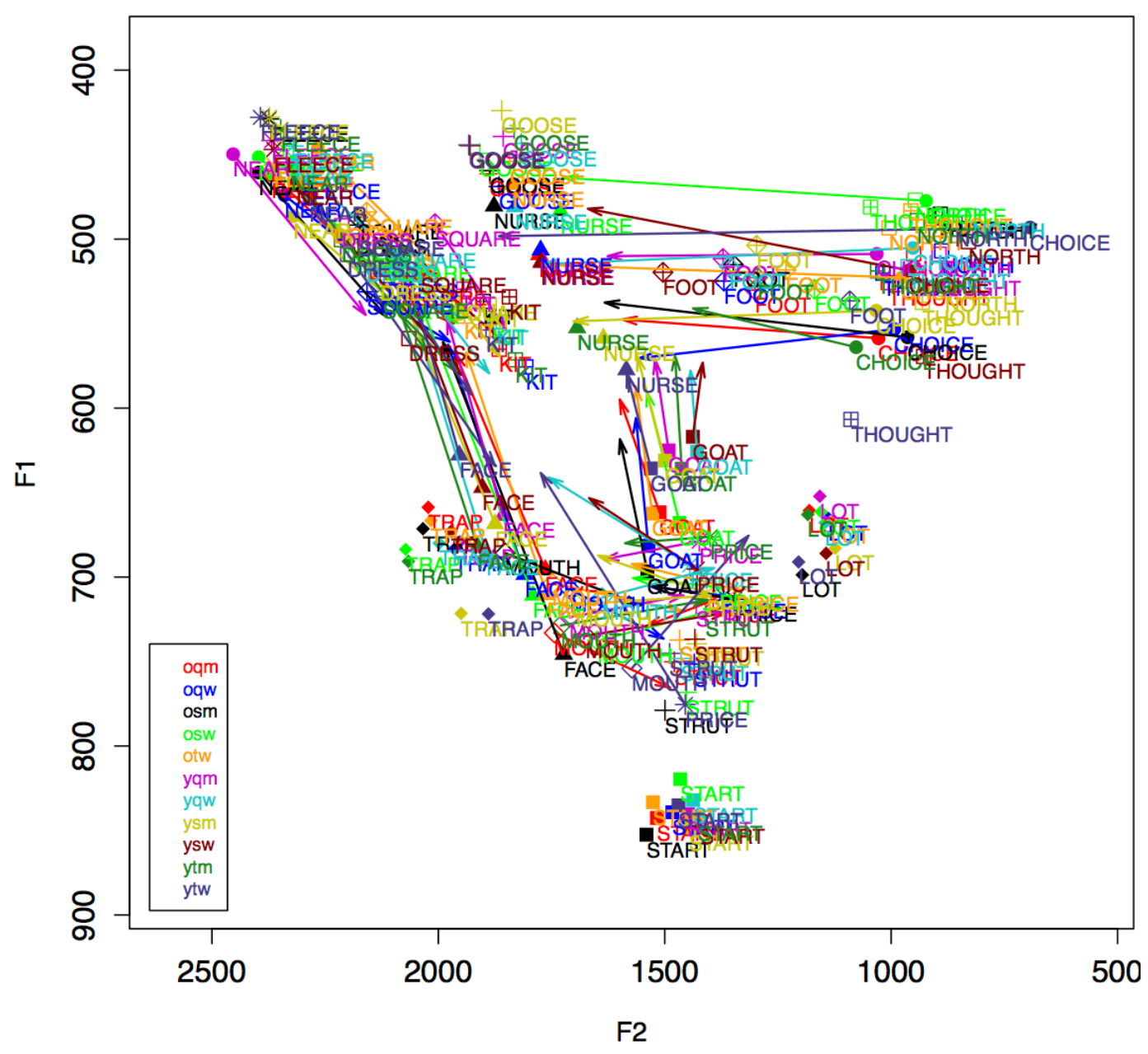

Figure 6.5: Overview of vowel space of New Zealand English

6.5 shows the means for each speaker group. Of course, this view is not much better there is too much overlapping information to make much sense of the plot. Nevertheless, a few points are worth mentioning before finding a better vantage point.

For all speakers, TRAP is fronted relative to the line between START and the high front vowels (in this view, it is difficult to tell FLEECE from NEAR in that corner of the plot). START is relatively isolated from the other vowels, and is also relatively tightly grouped, although older speakers seem in general to be slightly more peripheral than younger ones. LOT is also fairly isolated from other nearby vowels, although it is not so tightly grouped, as it shows more F1 (height) spread.

There are three regions of the plot that are particularly hard to characterise in any great detail. The high front corner of the vowel space has a lot of overlap between FLEECE, NEAR, DRESS, SQUARE, and KIT. Likewise, the high back corner comprises THOUGHT, NORTH, and CHOICE, to varying degrees of overlap. There is also a complicated region just above START, which includes STRUT and PRICE, and for some speakers, the onsets for GOAT and MOUTH. Because of these areas of overlap, it is useful to separate the vowels to get a clearer view of the vowel space. 


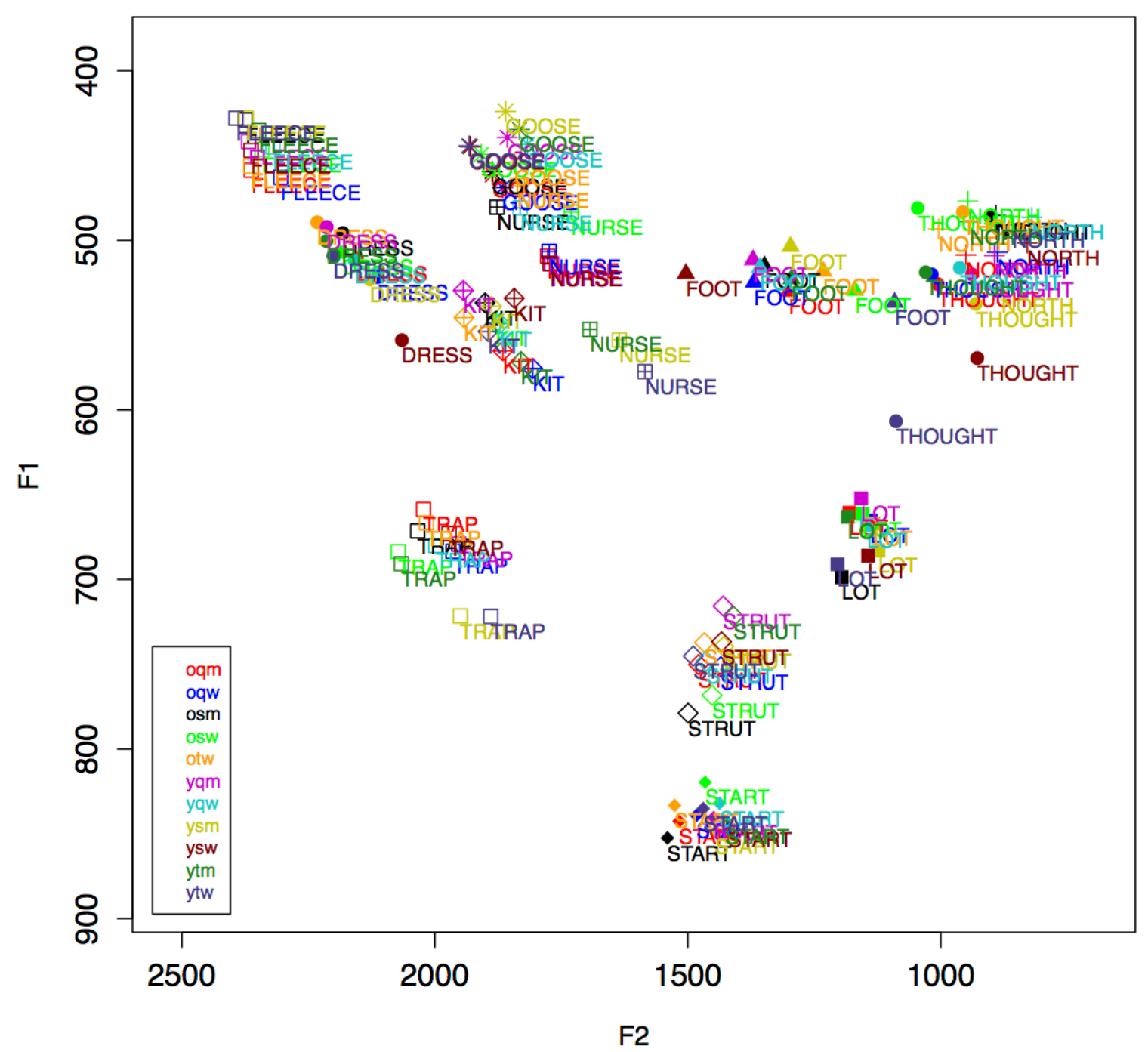

Figure 6.6: Monophthongs of New Zealand English

\subsubsection{Monophthongs}

The monophthongs on their own are shown in Figure 6.6, where it is considerably easier to see the trends in the data.

FLEECE, disentangled from the diphthong NEAR, seems to be about as compact in its spread as START. GOOSE likewise seems to be relatively stable. Most of the means for TRAP are also tightly grouped, but there are two that fall at some distance from the others. The plots for DRESS and KIT extend diagonally away from FLEECE in a fairly linear fashion, as does NURSE from GOOSE. The spread of STRUT is mostly vertical (F1), while that of FOOT is mostly horizontal (F2). LOT is fairly tightly grouped, but shows some F1 difference.

The high back corner continues to be a complicated area of the vowel plot. The decision to treat NORTH and THOUGHT as two separate vowels is discussed in §6.3.5.2, but for now it is enough to note that this decision seems justified by the data. For some 


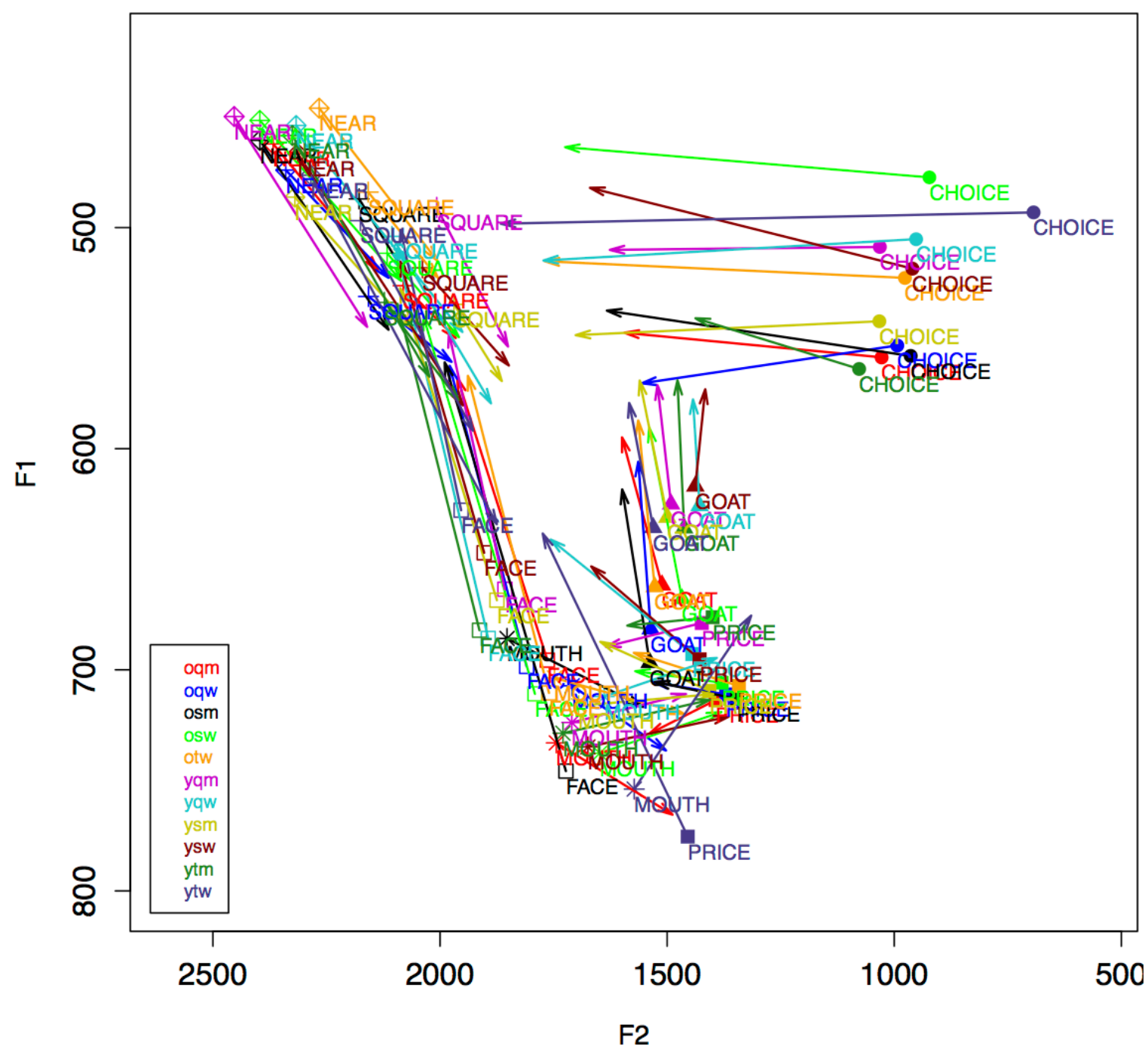

Figure 6.7: Diphthongs of New Zealand English

groups, the two vowels are nearly merged, while others have a substantial separation. This separation is particularly pronounced in vowel height, but some groups also show a frontness distinction.

\subsubsection{Diphthongs}

The diphthongs on their own are shown in Figure 6.7. Although this does not improve matters to the extent that Figure 6.6 did for the monophthongs, it nevertheless makes it easier to see patterns without the visual noise of the monophthongs.

We see that there is a considerable spread in the onset of FACE, along a diagonal line pointing towards NEAR, while the offglides end in the general region of SQUARE. It is interesting to note that NEAR and SQUARE seem overall firmly non-merged, although their off-glides are broadly parallel. The relationship between NEAR and SQUARE is discussed in detail in section §6.3.5.1. 
The lower vertex of the vowel triangle is densely packed with diphthongs. The spread of FACE starts in this area, intermixed with the onset of MOUTH. The offglide for MOUTH tends to end around where PRICE starts. It is interesting to note that neither MOUTH nor PRICE show an enormous raising in their offglides overall, although a few speaker groups (namely younger women) show a greater F1 increase. The spread of GOAT also starts in this corner of the plot, and extends upwards with most of the spread occurring in the F1 dimension (height).

CHOICE shows a great deal of variety, principally in F1, although there is some spread along F2 as well. However, the Ns for CHOICE are uniformly low (see Table 6.2 below), so it advisable to read this dispersion with caution. With as few as two or three tokens per speaker, the effect of chance differences in phonetic environment can be misleading to a description of the vowel more generally.

\subsubsection{Summary of the vowels for analysis}

Table 6.2 provides a summary of the vowels, including the mean Ns per speaker group, the range between the highest and lowest means for each vowel on both the F1 and F2 dimensions, as well as the dimension along which the vowel will be analysed in the following sections. Four vowels (FLEECE, GOOSE, LOT and START) are identified as stable vowels, meaning that they have relatively little spread in the vowel plot.

\begin{tabular}{|c|c|c|c|c|}
\hline vowel & mean $\mathbf{N}$ & range $\mathrm{F} 1(\mathrm{~Hz})$ & range $\mathrm{F} 2(\mathrm{~Hz})$ & dimension of analysis \\
\hline CHOICE & 15 & 87 & 384 & none: Ns too low \\
\hline DRESS & 226 & 70 & 167 & Euclidean distance (FLEECE) \\
\hline FACE & 318 & 118 & 231 & Euclidean distance (FLEECE) \\
\hline FLEECE & 388 & 35 & 88 & none: stable vowel \\
\hline FOOT & 40 & 33 & 413 & $\mathrm{~F} 2$ \\
\hline GOAT & 226 & 80 & 110 & $\mathrm{~F} 1$ \\
\hline GOOSE & 217 & 45 & 116 & none: stable vowel \\
\hline KIT & 167 & 46 & 137 & Euclidean distance (FLEECE) \\
\hline LOT & 271 & 47 & 81 & none: stable vowel \\
\hline MOUTH & 155 & 68 & 280 & Euclidean distance (START) \\
\hline NEAR & 34 & 40 & 187 & analysis with SQUARE \\
\hline NORTH & 88 & 53 & 185 & analysis with THOUGHT \\
\hline NURSE & 69 & 109 & 292 & Euclidean distance (GOOSE) \\
\hline PRICE & 354 & 99 & 113 & F1 \\
\hline SQUARE & 68 & 51 & 209 & analysis with NEAR \\
\hline START & 94 & 32 & 119 & none: stable vowel \\
\hline STRUT & 323 & 63 & 90 & $\mathrm{~F} 1$ \\
\hline THOUGHT & 48 & 126 & 187 & analysis with NORTH \\
\hline TRAP & 198 & 63 & 173 & Euclidean distance (START) \\
\hline
\end{tabular}




\subsubsection{Stable vowels}

Table 6.2 identified four vowels as 'stable': FLEECE, START, GOOSE, and LOT. This is not to say that there are no differences whatsoever between speaker groups, but rather that these vowels are generally clustered around a fairly restricted space, and they do not show clear trends that may highlight changes in progress, or some kind of social stratification. A vowel plot will be provided for each vowel to visualise the vowel dispersion, although there will be little discussion accompanying the plots. The grey vowels represent the mean across all speaker groups, to provide context for the plot for each vowel in isolation. Note that the means for all vowels across the gendered categories are listed in Appendix D.

For those vowels best analysed using the Euclidean Distance (ED) metric described in §6.2.6.1, these relatively stable vowels make for excellent reference points. In particular, FLEECE is used as a reference vowel for DRESS, FACE and KIT, while GOOSE is the reference point for NURSE, and START is used for TRAP. These vowels were chosen because, of the available candidates, they align best with the general trajectory of the non-stable vowels in question. 


\subsubsection{FLEECE}

Figure 6.8 provides the vowel plot for FLEECE. The space occupied by FLEECE coincides with the mean onset for NEAR, which is unsurprising given that the NEAR diphthong in NZE is characterised as starting at FLEECE and centring. The phonetic symbol used by many phoneticians in New Zealand (e.g., Bauer, Warren, Bardsley, Kennedy \& Major 2007) for the NEAR diphthong is /is/. Although not a short vowel, FLEECE has been implicated in the short front vowel shift of NZE (e.g., Maclagan \& Hay 2007; Maclagan \& Hay 2004; McKenzie 2005), affected by DRESS raising. In the data in this project, however, DRESS is not raised enough to interfere with FLEECE.

McKenzie (2005) discusses the diphthongisation of FLEECE in response to DRESS raising, and notes that it is more pronounced in wordlist data than it is in spontaneous speech. The tokens of FLEECE here are not appreciably diphthongised, presumably due in part to the relatively lower DRESS, and also perhaps due to the conversational nature of the data.

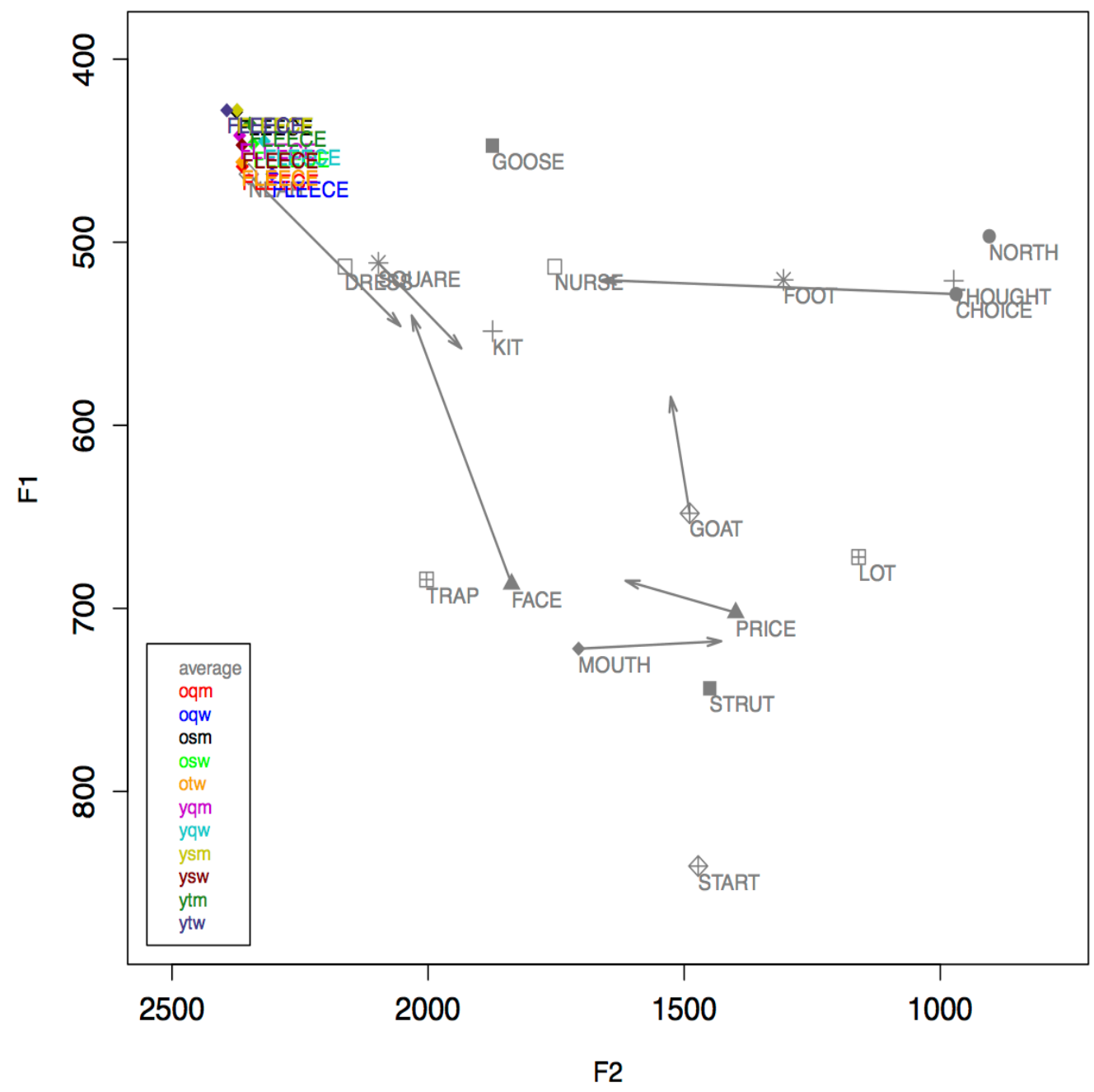

Figure 6.8: Vowel plot for FLEECE 


\subsubsection{START}

The vowel plot for START is given in Figure 6.9. START is often described (e.g., Bauer \& Warren 2004a; Watson, Harrington \& Evans 1998; Maclagan 1982) as differing from STRUT only in length, i.e., / $\mathfrak{e} / /$ and $/ \mathfrak{e} /$, respectively. This is clearly not the case in this data, where we see a clear difference in F1 between the two vowels (see $§ 6.3 .5 .3$ for a more detailed discussion of this pair).

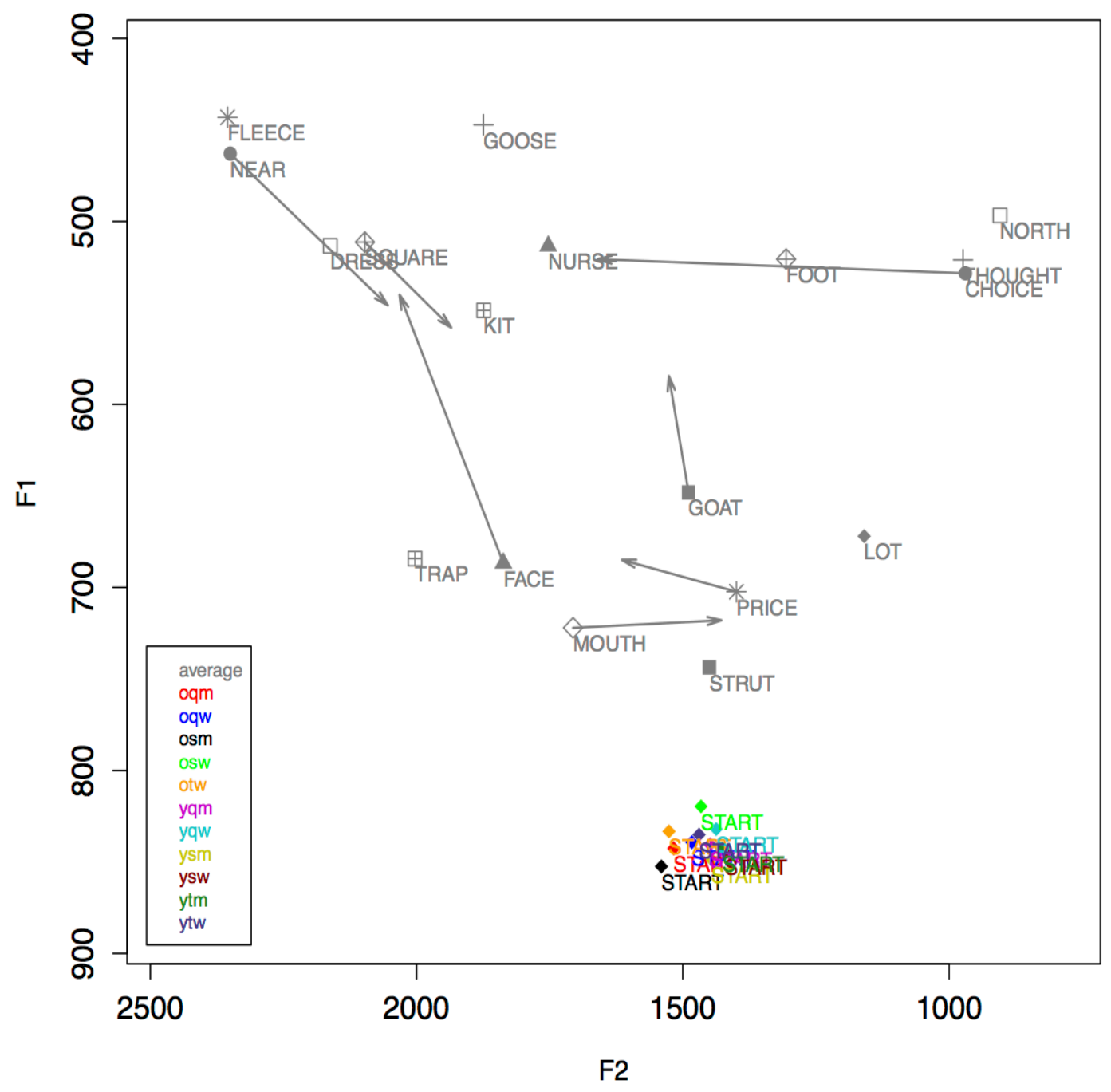

Figure 6.9: Vowel plot for START 


\subsubsection{GOOSE}

Figure 6.10 provides the vowel plot for GOOSE. The values for GOOSE in this data are somewhat more fronted (or potentially less rounded) than in previous surveys of NZE vowels (e.g., Hay, Maclagan \& Gordon 2008: 21); unfortunately, FAVE was inconsistent in producing F3 values, so the effect of rounding is impossible to determine in this data. This may be due to either the location of the recordings, or to the spontaneous nature of this data.

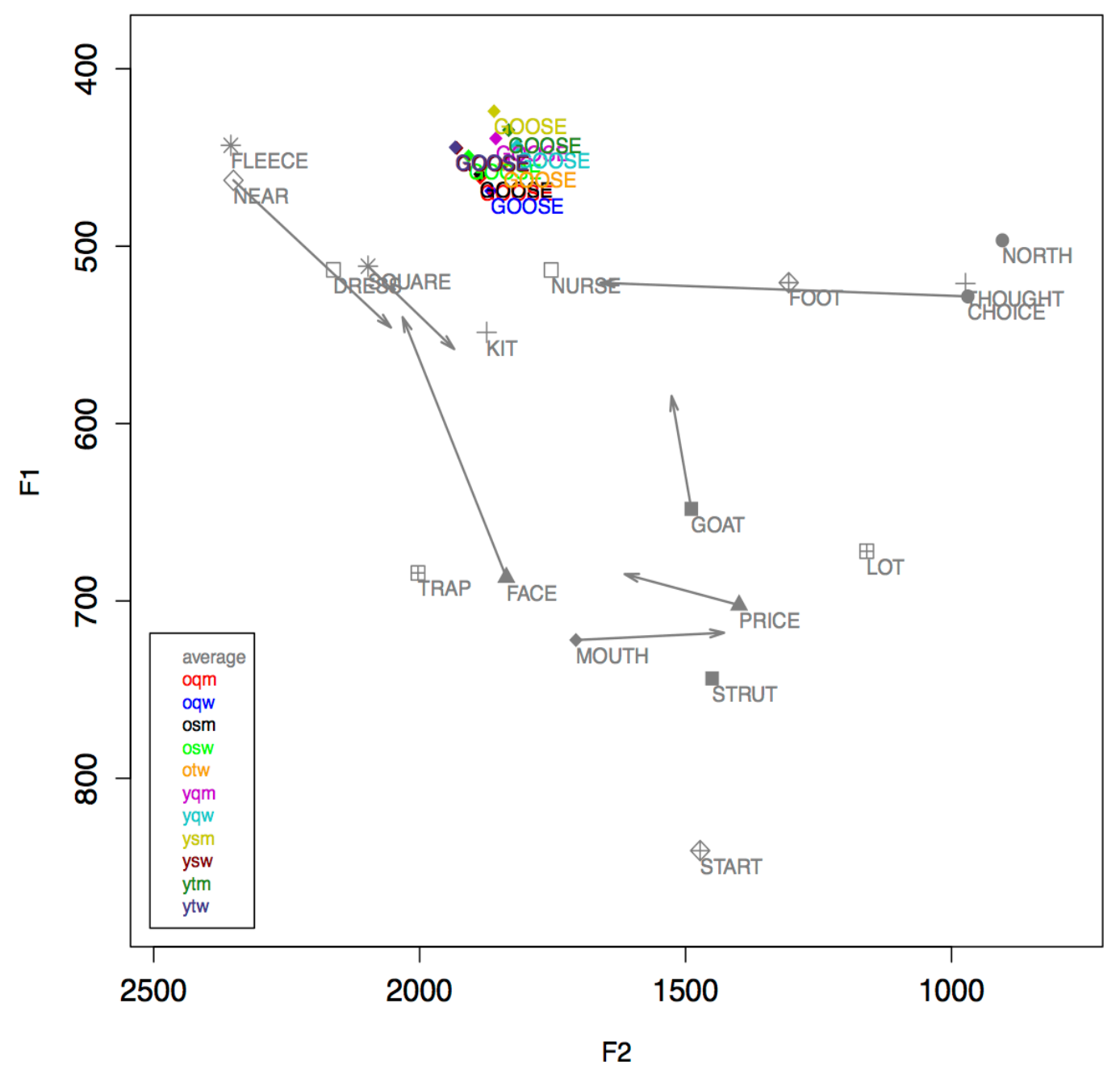

Figure 6.10: Vowel plot for GOOSE 


\subsubsection{LOT}

Figure 6.11 shows the vowel plot for LOT. While there is some suggestion of an F1 spread for LOT, the range is smaller than in most other vowels (see summary in Table 6.2 ), and relative to the vowel system as a whole, the grouping is actually quite compact. There are no clear trends along social lines.

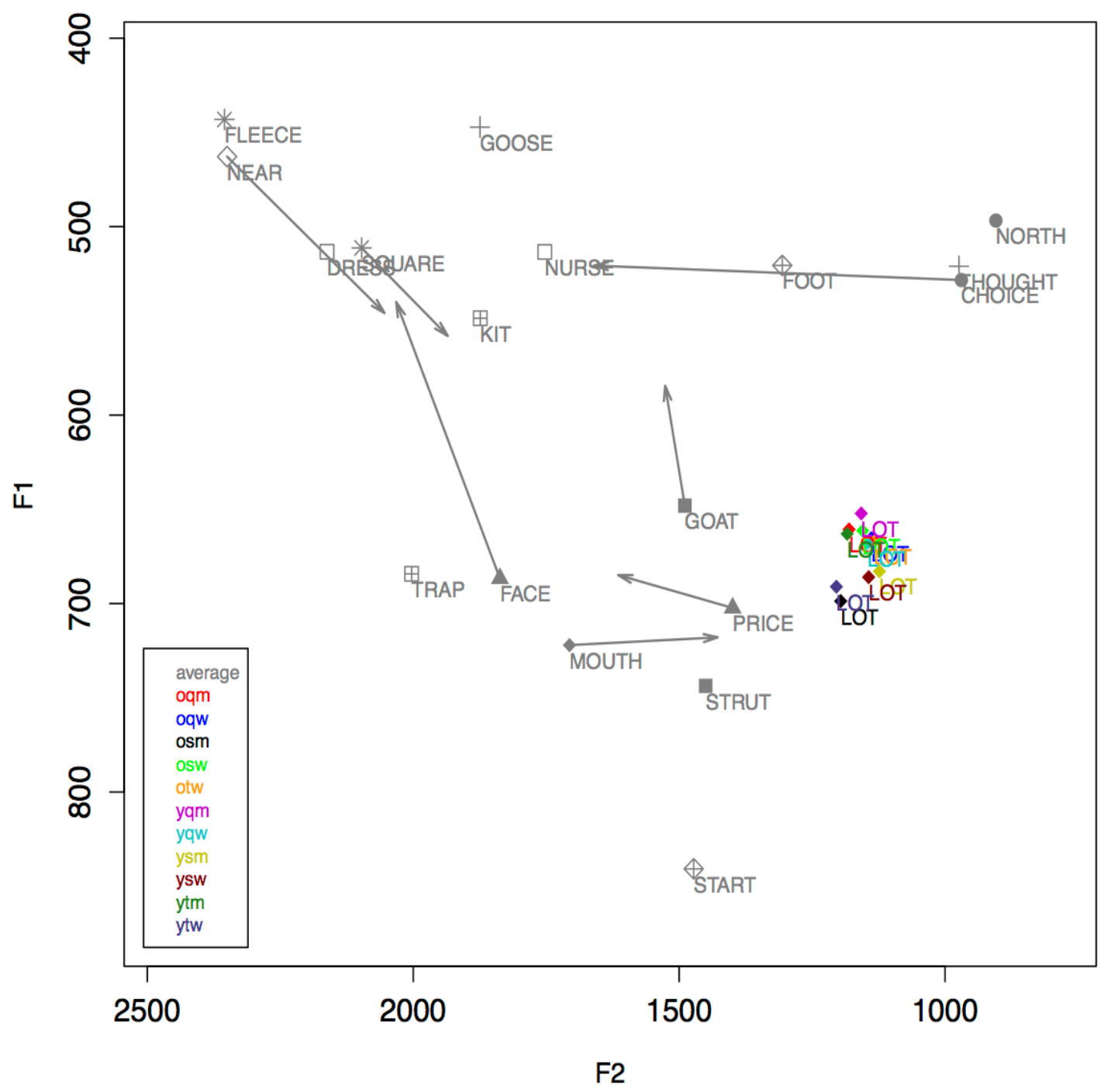

Figure 6.11: Vowel plot for LOT 


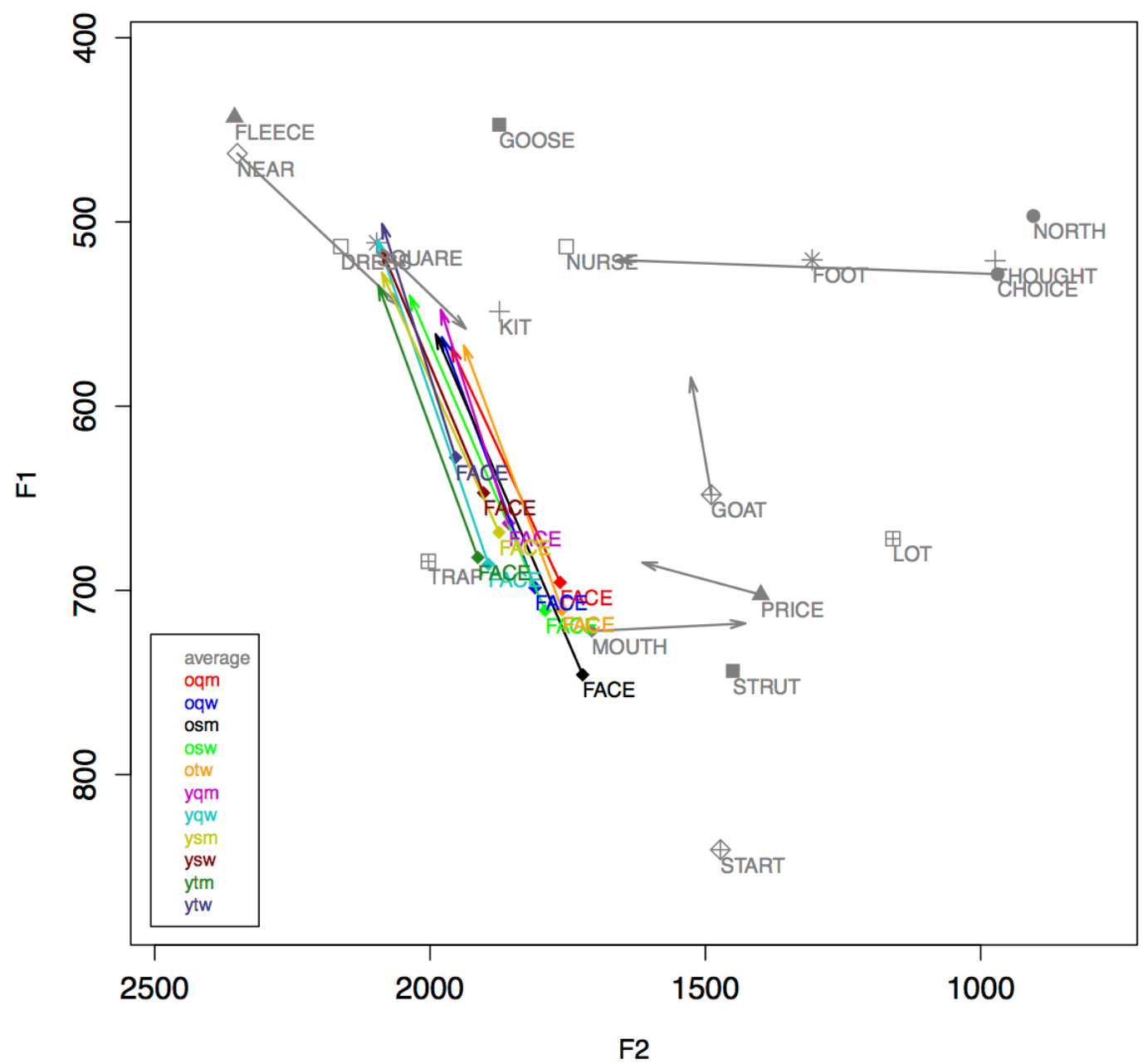

Figure 6.12: Vowel plot for FACE

Table 6.3: Multivariate analysis of FACE onset ED (social factors only)

\begin{tabular}{cccc}
\hline Gender & Coef & $\mathrm{N}$ & Mean \\
\hline ytw & -125.827 & 178 & 455 \\
ysw & -65.421 & 463 & 511 \\
ytm & -45.292 & 357 & 520 \\
yqw & -42.400 & 239 & 532 \\
ysm & -41.318 & 342 & 540 \\
yqm & -19.358 & 359 & 553 \\
\hline oqw & 27.533 & 232 & 612 \\
osw & 59.642 & 350 & 634 \\
otw & 67.440 & 263 & 659 \\
oqm & 73.718 & 285 & 650 \\
osm & 111.284 & 427 & 706 \\
Range & 237.111 & Sig. & $p=0.001$ \\
\hline Also retained as significant in model: \\
- Preceding segment $(p<0.001)$ \\
- Following segment $(p<0.001)$ \\
\hline \multicolumn{4}{c}{} \\
\hline
\end{tabular}




\subsubsection{Vowels showing a clear trend}

The vowels discussed in this section are grouped according to patterns or similarities observed in the data. Each is presented with a plot showing all eleven gendered identity groups for that vowel, with the overall means for the other vowels provided (from Figure 6.4) to give a sense of the vowel's position in the vowel space as a whole; the means for each vowel under discussion are provided in Appendix D. Additionally, each vowel is accompanied by its multivariate analysis, which is the basis for the discussion. The multivariate analyses are presented to highlight any gender effects, but the significant linguistic constraints are also noted in each table. This allows the reader to contextualise the strength of the social variables as predictors.

\subsubsection{FACE and GOAT}

FACE and GOAT are the two diphthongs in this data that are unequivocally closing diphthongs for all groups of speakers. Both also show a general spread in the height of the onsets, with GOAT's differences principally along the F1 dimension, and FACE's differences lying on a line extending roughly between FLEECE and START.

\subsection{FACE}

The onset for the FACE vowel has been analysed using Euclidean distances (EDs) from the stable vowel FLEECE, as this is the line along which the vowels are spread out. A larger ED therefore means that the vowel is further from FLEECE, and can be thought of as "lower" along the reference line. A smaller ED corresponds to a "higher" vowel. Figure 6.12 provides a plot of FACE, and Table 6.3 gives the multivariate analysis of gendered identity. Note that here, a negative coefficient means that the group has a smaller ED, which corresponds to a higher realisation of the vowel.

The multivariate analysis shows a clear age split in the data, with younger speakers having universally higher onsets for FACE than older speakers. Among younger speakers, there are roughly four groups: queer men (who have the lowest onsets); then a cluster consisting of straight men, queer women, and trans men (whose mean ED is around 20 coefficient units higher than queer men); then straight women (again, around 20 units higher than the previous group); and finally, trans women (whose coefficient is nearly twice that of straight women). If we temporarily set aside the trans speakers, we see that women are generally higher than men, but the effect is not enormous; it is also interesting to note that queer women and straight men are closely patterned, while queer men and straight women have a considerably bigger gap between them. The trans men are patterning with queer women and with straight men, making it difficult to determine whether FACE carries social meaning (i.e., it is ambiguous whether they are maintaining a previously-acquired queer-women articulation, or whether they have equally adapted to a straight-male articulation). The trans women have a substantially more raised onset than anyone else, which could be taken as a sign that they have adapted their articulation 


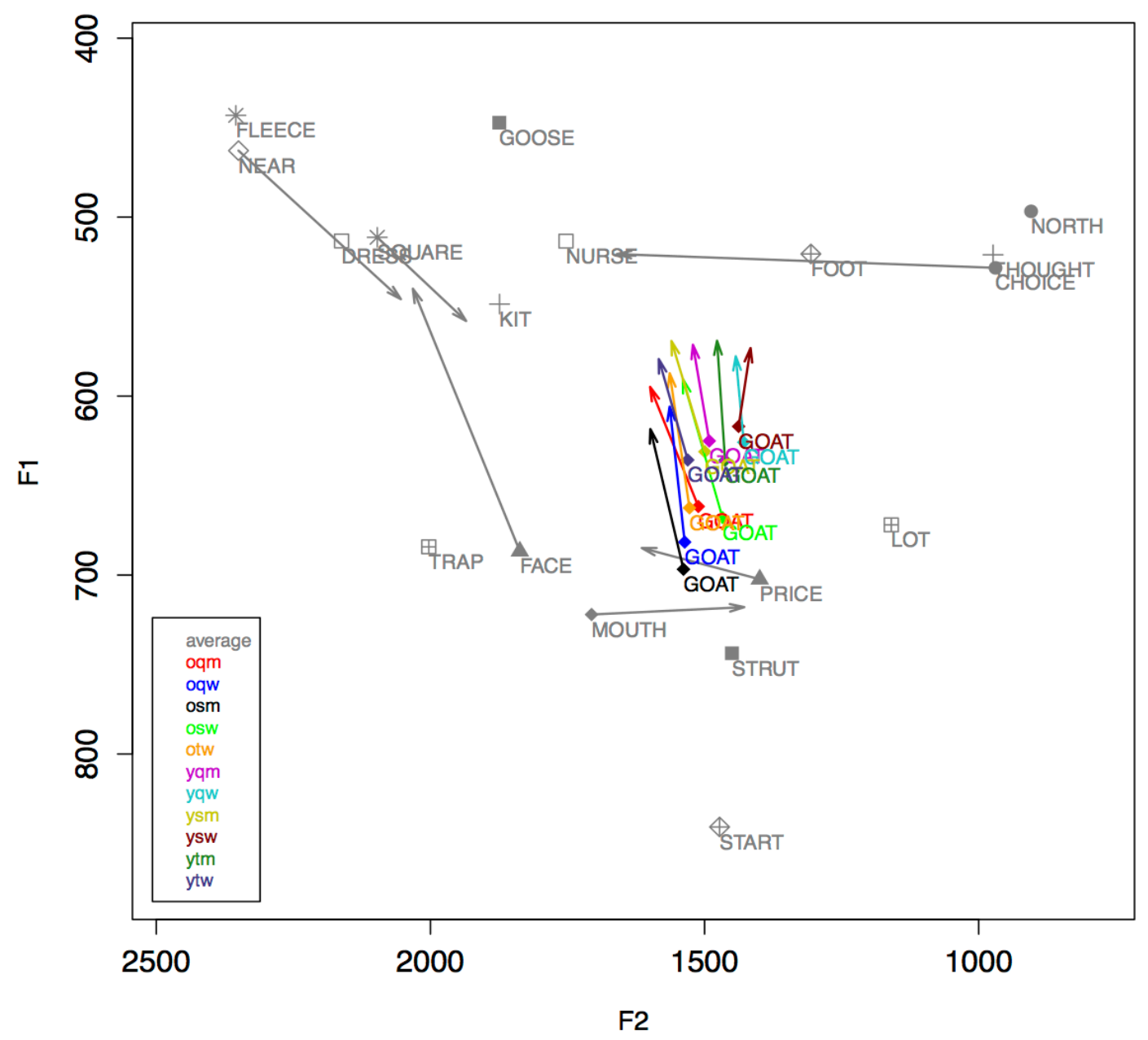

Figure 6.13: Vowel plot for GOAT

Table 6.4: Multivariate analysis of

GOAT onset F1 (social factors only)

\begin{tabular}{cccc}
\hline Gender & Coef & $\mathrm{N}$ & Mean \\
\hline ysw & -26.600 & 301 & 617 \\
ysm & -23.219 & 228 & 631 \\
yqm & -22.403 & 279 & 625 \\
yqw & -19.678 & 190 & 626 \\
ytm & -15.407 & 308 & 636 \\
ytw & -11.241 & 132 & 636 \\
\hline oqm & -0.577 & 194 & 662 \\
otw & 17.095 & 152 & 662 \\
osw & 19.036 & 244 & 668 \\
oqw & 37.039 & 171 & 681 \\
osm & 45.959 & 285 & 697 \\
Range & 72.559 & Sig. & $p<0.001$ \\
\hline Also retained as significant in model: \\
- Preceding segment $(p<0.001)$ \\
- Following segment $(p<0.001)$ \\
\hline \multicolumn{4}{c}{} \\
\hline
\end{tabular}


to a more feminine standard and have overshot the mark, but it could also be that these speakers are orienting to a different speech norm altogether (\$2.3.2.3).

Among older speakers, there are three groups: straight men (with a markedly lower onset than anyone else); then a cluster of straight women, trans women, and queer men; and finally queer women (with the most raised onset). Generally, women have higher onsets than men, although it is interesting to note that with the older speakers, queer women and straight men are maximally separated, unlike the pattern observed in the younger speakers. The older trans women pattern with both straight women and queer men, so it is not possible to infer the degree to which FACE is a marker of gender for this age group.

The trajectory for the offglides is nearly parallel for all speakers, and ends in the general vicinity of DRESS/SQUARE/KIT.

\subsection{GOAT}

Although the onset for GOAT shows variation in both height and frontness (Figure 6.13), the bulk of the difference seems to be with respect to F1, and so this is the dimension along which it is analysed here. Using a Euclidean distance (ED) measurement here would not be appropriate, as the overall trend is slightly curved towards the back of the mouth for those speakers with a higher onset, rather than the straight line that motivates recourse to ED. If we were to approximate this curve (which is not particularly steep) as a straight line, the nearest stable vowel we could reasonably use as a reference point is START - which is nearly directly below GOAT - so we would, in effect, be analysing the variation along the F1 axis anyway. Table 6.4 provides the multivariate analysis of F1, where a lower coefficient is associated with a higher articulation.

As we saw with FACE, there is a clear age difference, with younger speakers having universally higher onsets for GOAT than older speakers, although the range in coefficients is much smaller with GOAT, suggesting that the gendered effect is not as strong.

The majority of the younger speakers have very similar coefficients, with only the trans speakers having overall lower onsets than the other groups. The differences between the queer and straight speakers are too small to read much social meaning into this pattern, and even the younger trans women - although having the lowest onsets of their age cohort - have a coefficient only $15 \mathrm{~Hz}$ below that of straight women. It is difficult with this distribution of coefficients to argue the case for a gendered indexicality for GOAT among younger speakers.

Among older speakers, however, we see a more pronounced difference. Queer men have the most raised onset, while straight men have the lowest, with queer women again patterning more like straight men. Trans women pattern most closely with straight women, suggesting that among older speakers at least, GOAT carries enough socially gendered information that it is worth raising the onset to produce a more feminine-marked 
variant.

Because we had earlier noted that the trajectory of the GOAT onset is slightly curved upwards and towards the back of the mouth, it is worth noting that the younger speakers are generally also those with the backest realisation, suggesting that as well as raising, GOAT is moving back. With regard to the offglides, although they are more or less parallel across all speaker groups, it is interesting to note that the glides are generally shorter for younger speakers than for older ones.

\subsection{Some observations on FACE and GOAT}

The onsets for both FACE and GOAT show a clear and consistent age split, with younger speakers producing higher variants than older speakers. This suggests that, overall, both vowels are raising over time.

With FACE, the picture is quite straightforward: younger speakers and women have the higher variants. This is a classic pattern for Labovian change-from-below. Indeed, the behaviour of younger trans men and older trans women - who are producing variants more in line with their childhood socialisation than with their actual, current gendered identities - further supports the idea that the observed differences are due to general linguistic change, rather than identity work.

GOAT presents a slightly more complicated picture, one that suggests that the ongoing change is more gendered. For younger speakers, gendered differences are generally neutralised, with trans speakers producing more queer-like variants than straight. For older speakers, we see a much more gendered division, with straight and queer men distancing themselves from each other.

Thus although both FACE and GOAT show age-related raising of their onsets, GOAT seems to be more amenable to gendered identity work than FACE, at least among older speakers.

\subsubsection{DRESS and KIT}

Both DRESS and KIT are measured using Euclidean Distance (ED) with FLEECE as a stable reference vowel. Both are relatively high front vowels, but they are clearly distinct phonemes.

KIT is a particularly iconic vowel of New Zealand English. It acts as a shibboleth with regards to Australian English, the closest neighbouring variety and one that shares many of the same phonetic features. When speakers of northern hemisphere varieties of English caricature the New Zealand accent (and, indeed, when some New Zealanders and Australians do, as well), KIT-retraction tends to be exaggerated: fush and chups vs feesh and cheeps. It seems, then, to be strongly associated with some degree of national identity, but there is still room for variation between different New Zealand identities.

By contrast, DRESS on its own seems to be the subject of very little direct commentary (unlike KIT), and seems to be a fairly nondescript vowel in terms of the 
salience of New Zealand English, although it can be confused with KIT (e.g., six/sex) to some speakers of other varieties. It is part of a push chain assumed to have started with TRAP (e.g., Langstrof 2006; Maglagan and Hay 2004; Gordon et al. 2004), but this raising does not seem to be stigmatised (Maclagan, Gordon \& Lewis 1999). Maclagan \& Hay (2004) have found that women raise DRESS to the point of overlap with FLEECE in read word-list data. This finding was not replicated in the ATC data.

\subsection{DRESS}

The DRESS vowel has been analysed using Euclidean distances (EDs), using FLEECE as a reference vowel. A larger ED measurement means that the vowel is farther from FLEECE, and a smaller one means it is closer (i.e., higher and fronter). The plot for DRESS is presented in Figure 6.14, and the multivariate analysis of the social factors is summarised in Table 6.5, organised by age group for easier discussion. Note that the age groups are presented side-by-side here, in contrast with Tables 6.3 and 6.4, because there is distributional overlap (i.e., the ranges of the coefficients and means overlap) for DRESS, while they did not for the previous vowels.

It is interesting to note that the younger speakers have coefficients more strongly lowering the articulation than older speakers do, which suggests that the raising pushchain associated with TRAP movement may be reversing. Younger straight women have the lowest DRESS overall, which would be consistent with a Labovian analysis of sound change. If RP is still a reference for NZE, this could signal that these women are moving away from signalling [+NZ] and towards signalling [+posh]. Alternatively, it could be that the over-crowding in the high front corner of the NZE vowel space means that DRESS cannot feasibly be raised any further without either losing comprehensibility (i.e., merging with FLEECE) or triggering another substantial chain-shift; this possibility will be revisited in $\S 7.2 .1$.

Among younger speakers, we note that cis women have the most lowered DRESS, with straight women considerably lower than their queer counterparts. Cis men make up the middle of the younger range, and trans speakers have the highest onsets. As noted elsewhere, the younger trans women in this project may be orienting to a different variety of English, which may go some way to explaining their lower-than-expected coefficient. The trans men, however, are of the same ethnicity and broad demographic categories as their cis peers in this corpus, so their pattern may be evidence of an overcorrected masculinity; or, if younger speakers are involved in a reversal of previous trends, these trans men may be producing a conservatively-raised DRESS. The fact that they are producing something on the masculine end of the continuum suggests that DRESS is potentially salient with respect to gender in the speech community, at least among younger speakers.

For the older speakers, the picture is less straightforwardly gendered. There is a clear split between queer and straight speakers, with queer participants favouring a lowered DRESS and straight ones favouring a more raised vowel. If we take the patterns 


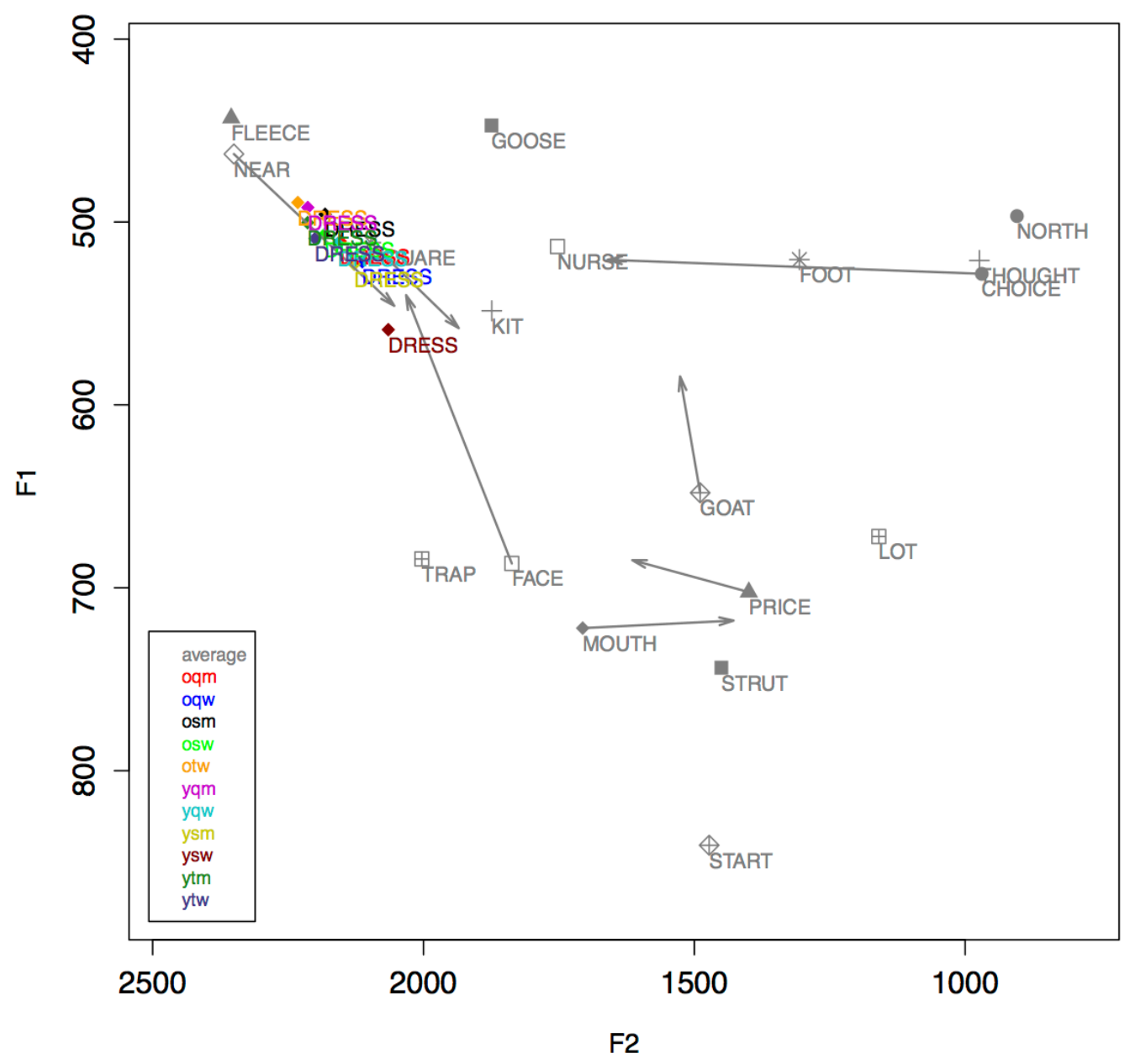

Figure 6.14: Vowel plot for DRESS

Table 6.5: Multivariate analysis of DRESS ED (social factors only), organised by age

\begin{tabular}{ccc|ccc}
\hline $\begin{array}{c}\text { Younger } \\
\text { speakers }\end{array}$ & Coef & Mean & $\begin{array}{c}\text { Older } \\
\text { speakers }\end{array}$ & Coef & Mean \\
\hline ytm & -43.131 & 218 & osw & -51.208 & 255 \\
ytw & -34.933 & 245 & otw & -45.871 & 213 \\
yqm & 8.004 & 271 & osm & -39.743 & 234 \\
ysm & 19.976 & 290 & oqm & 27.763 & 309 \\
yqw & 31.536 & 305 & oqw & 42.235 & 309 \\
ysw & 85.552 & 377 & & & \\
Range & 128.683 & \multicolumn{5}{l}{ Range } & 93.443 & \\
\hline \multicolumn{7}{l}{ Range overall: 136.760} \\
\hline
\end{tabular}


of the younger speakers as evidence that DRESS is lowering, then among the older cohort, queer speakers are the in the lead. If we assume that the apparent reversal observed among younger speakers is a very recent innovation, then we could also argue that queer speakers are at the trailing edge of the previous DRESS-raising trend. Whatever the reason for this difference between straight and queer speakers, the result is that queer and straight women are maximally differentiated within their age group. Because the older trans women pattern with both straight men and women, it is not possible to infer the gendered indexicality of DRESS among older speakers, so we are also not able to determine if this observed difference between queer and straight women is socially-interpretable, either.

\subsection{KIT}

The KIT vowel has been analysed using Euclidean distances (EDs) from FLEECE. A larger ED measurement means the vowel for that speaker group is farther from FLEECE - lower and further back. A smaller ED measurement means that the vowel is higher and fronter. The plot for KIT is presented in Figure 6.15, and a summary of the social factors retained in the multivariate analysis is presented in Table 6.6.

The best model of variation for KIT retained an interaction of age and identity as the relevant social categories, although as the p-value and small overall coefficient range suggest, the effect is not particularly strong. Among younger speakers, straight and trans participants have a more lowered and retracted KIT, while queer speakers have the highest and frontest; among older speakers, the effect is reversed. Interestingly, the biggest difference between groups is between older and younger queer speakers, and the smallest difference is between older and younger straight speakers. The fact that younger trans speakers are best modelled together, despite the difference in their gendered identities, suggests that KIT is not socially-interpretable with respect to gender. It may, however, be indexical of participation in the queer community, or rather queer communities; the difference between older and younger queer participants may be reflective of social changes in New Zealand between the pre- and post-HLR periods (see $\S 2.2 .3)$.

KIT may be less indexical of gender in part because its available salience 'bandwidth' is dwarfed by the effect of its [+NZ] indexicality. It is interesting to note that younger queer speakers have a less iconically-New Zealand vowel than their older counterparts: this may suggest an affiliation with a global queer community for younger speakers, or at least a globally-Westernised one (e.g., Altman 1996; Oswin 2006), which may be pulling them away from such a strong [+NZ] vowel.

\subsection{Some observations on DRESS and KIT}

The patterns we observed with DRESS and KIT paint a complex picture of the high front corner of the vowel space. Younger women are leading in the lowering and retraction of DRESS, which brings it into close proximity with KIT, but we do not see a 


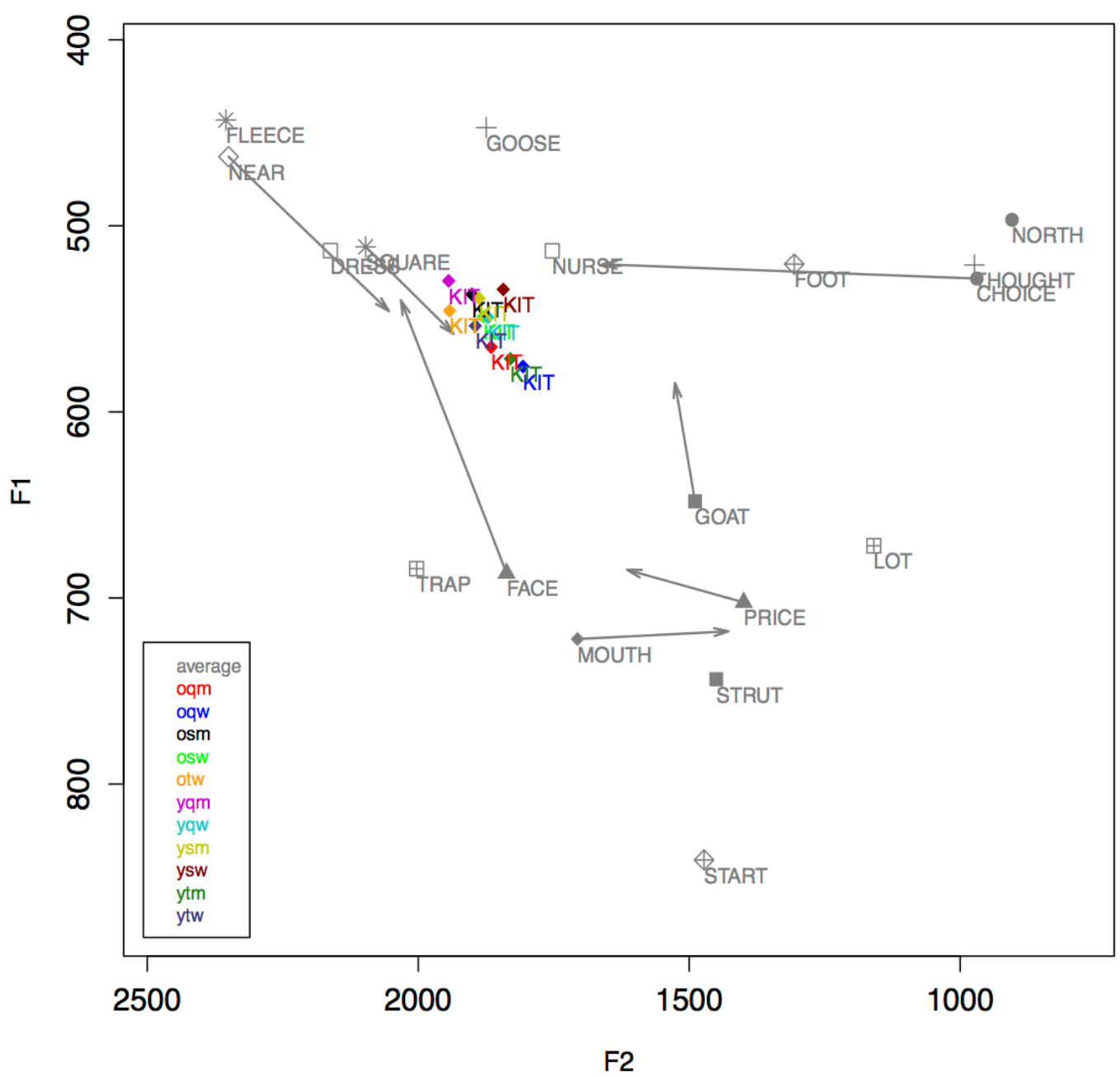

Figure 6.15: Vowel plot for KIT

Table 6.6: Multivariate analysis of KIT ED (social factors only)

\begin{tabular}{lccc}
\hline Age and identity & Coef & $\mathrm{N}$ & Mean \\
\hline younger queer & -37.658 & 383 & 464 \\
older trans & -25.676 & 107 & 450 \\
older straight & -11.982 & 333 & 497 \\
younger straight & 11.982 & 436 & 519 \\
younger trans & 25.676 & 268 & 533 \\
older queer & 37.658 & 311 & 553 \\
\multicolumn{1}{c}{ Range } & 75.316 & Sig. & $p=0.032$ \\
\hline Also retained as significant in model: \\
- Preceding segment $(p<0.001)$ \\
- Following segment $(p<0.001)$ \\
\hline
\end{tabular}


clear corresponding push-chain reaction with the distribution of KIT. This may mean that the lowering of DRESS is simply too recent to have had much of an effect on KIT, or it could be that because of its iconic New Zealand status, KIT is relatively fixed in the geometry of the vowel space.

The variation that we $d o$ see with KIT is potentially best explained through an orientation towards or away from New Zealand. Younger queer people are arguably more attuned to the international westernized 'global gay', having grown up in a time when western - or more precisely, American - queer identities have saturated the media landscape, and popular discourses on sexuality and identity are heavily biased towards US and UK experiences and narratives. In contrast, for the older queer participants, the development of their own identities may have been politically influenced by international affairs (e.g., legal challenges in the UK, the Stonewall Inn riots in the US) but was nevertheless a grass-roots, New Zealand exercise.

This perspective may also go some way to explaining the slightly weaker (and reversed) effect observed among trans participants with KIT. For the older trans women (recall that there are no older trans men in this corpus), social resistance to trans identities within New Zealand may have turned their gazes outward, to higher-profile people such as Christine Jorgensen (a famous American trans woman from the 1950s, who was explicitly mentioned by two of the three older trans women in this corpus), which may have created an affective distance from a strong [+NZ] identity. For the majority of the younger trans participants, however, there is a sense that New Zealand is a good place to be trans: it's not an idyllic paradise, but the (generally white and educated) participants that I have worked with feel relatively safe and supported by the wider community, so they may have a stronger affiliation with New Zealand.

\subsubsection{FOOT and NURSE}

The FOOT and NURSE vowels in New Zealand English are an interesting pair to consider together. Kennedy (2006) reports that for some speakers, the space of THOUGHT overlaps with that of FOOT such that the distinction between them is often one of duration, while Bauer \& Warren (2004a) and Warren (2004) observe that FOOT has been fronting for some time. As it fronts, it moves towards the space occupied by NURSE and GOOSE.

In this data, we see that, as FOOT stretches out along the F1 dimension, NURSE is extending down and back from GOOSE. This follows the general pattern of a pushchain, with NURSE retreating in the face of an encroaching FOOT.

\subsection{FOOT}

Although there are some height differences between groups of speakers, on the whole FOOT seems to show the greatest differences in frontness/backness (or potentially rounding, which we are not able to determine from F2 alone), so this is the dimension we will consider. Figure 6.16 shows the vowel plots for FOOT, and Table 6.7 provides a 


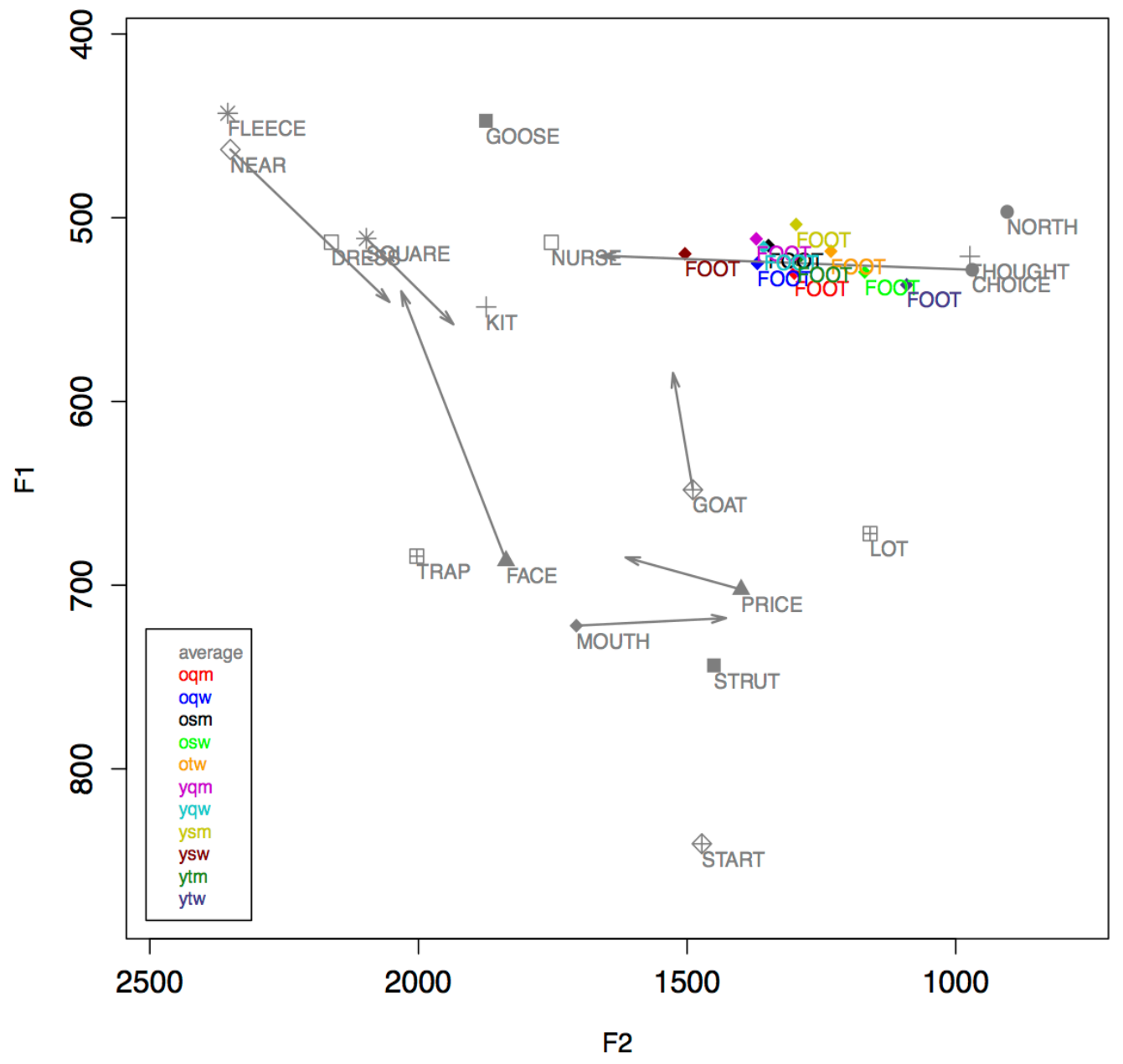

Figure 6.16: Vowel plot for FOOT

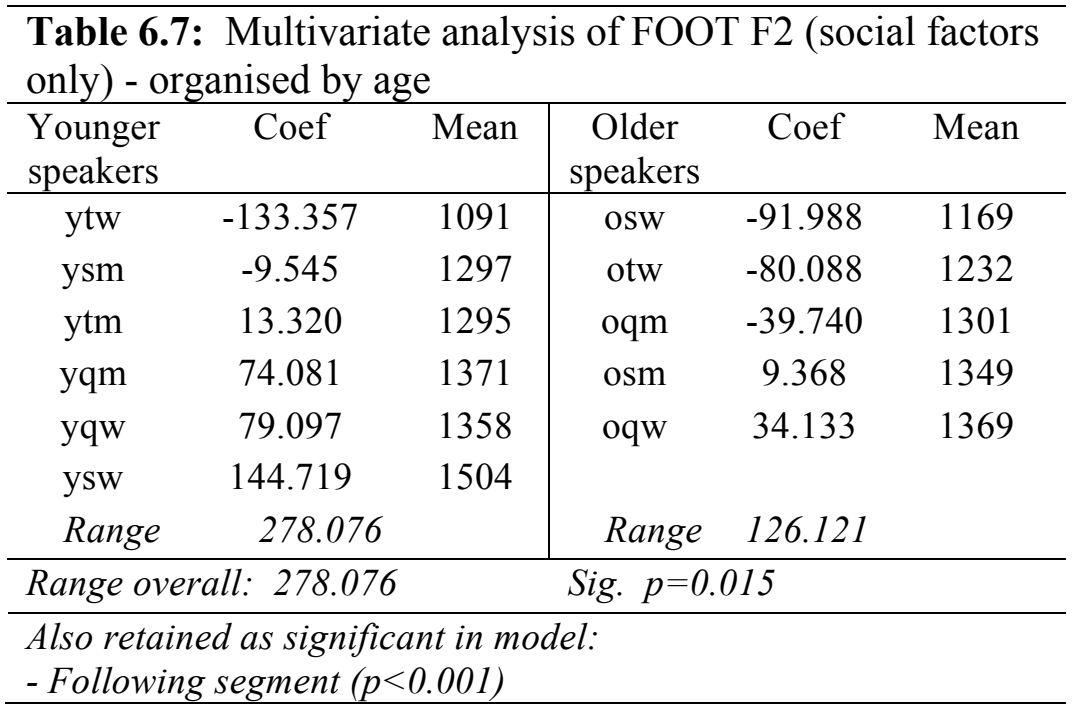


summary of the multivariate analysis. Because of the orientation of the F2 axis, a smaller F2 value indicates a backer variant (as does a negative coefficient in the multivariate analysis), and a larger F2 value (positive coefficient) indicates a more fronted one.

Generally, younger speakers tend to have more fronted realisations than older speakers do, although there is some overlap. The overall most fronted speakers by a considerable margin are younger straight women, followed by younger queer women and younger queer men, while the most retracted speakers are older trans women, older straight women, and younger trans women. As discussed elsewhere (see §2.3.2.3), ethnicity likely explains the extremely backed variant produced by younger trans women.

With younger women having more fronted realisations, this looks a fairly straightforward change in progress, continuing the fronting that Bauer \& Warren (2004a) and Warren (2004) noted previously. It is interesting that among older speakers, straight women are the most resistant to this change, as we might expect to find them leading in their age cohort. However, queer women are the most fronted in the older group, so it may be that resistance to FOOT fronting is a marker of heterosexual femininity in this age cohort.

Both younger trans men and older trans women - who are ethnically similar to their cissexual peers, unlike the younger trans women, and are therefore more suitable points for direct comparison - are patterning with their identified gender: the trans men are firmly in the masculine band for their age group, and the trans women parallel their straight cissexual counterparts. This suggests that FOOT has clear gendered salience in the speech community, which is further bolstered by the fact that it is also available for expressing sexuality among older women.

\subsection{NURSE}

The NURSE vowel is analysed using Euclidean Distances (EDs), with GOOSE as a stable reference vowel. A lower ED value (and a negative coefficient in the multivariate analysis) corresponds to a position closer to GOOSE and therefore articulated at a higher position, while a larger ED (and a positive coefficient) indicates a position farther from GOOSE, and a lower and more retracted articulation. Figure 6.17 shows the vowel plot for NURSE, and Table 6.8 gives the multivariate analysis of the ED.

Overall, younger speakers tend to show a lowering effect on their articulation (as seen through the positive coefficients), suggesting a change in progress. However, unlike what we would expect in a classical sound change-from-below, it is younger straight men who are leading. Younger trans women have the lowest overall production, but as discussed elsewhere (see $\$ 2.3 .2 .3$ ), this may be because they are orienting to a more Pasifika variety of Auckland English. Queer speakers have the most raised realisation among the younger cohort; it is possible then that NURSE lowering indexes a particular style of young masculinity that these queer speakers are distancing themselves from, but that trans men are at least somewhat invested in.

Among older speakers, we see that women have a more lowered articulation than 


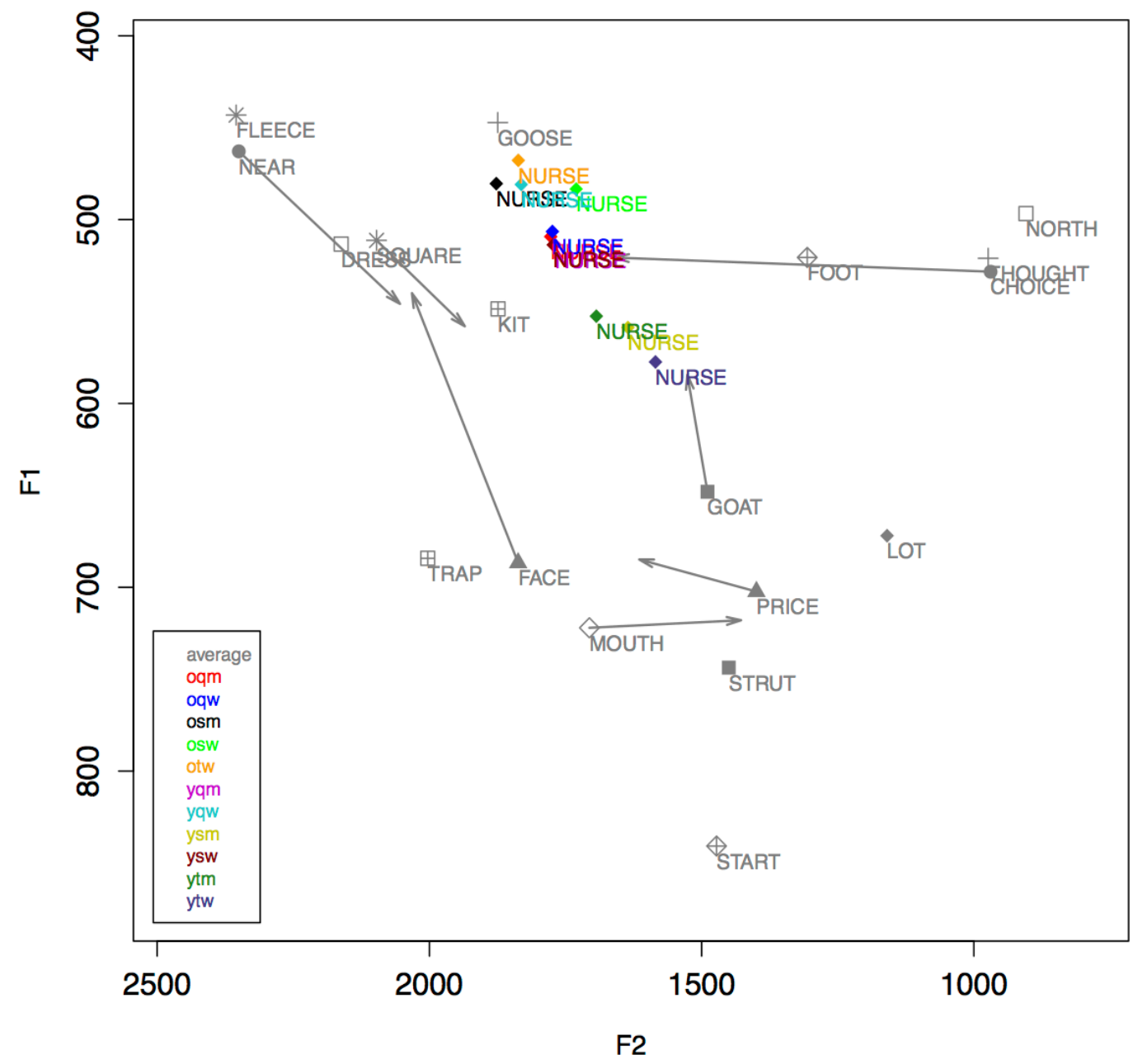

Figure 6.17: Vowel plot for NURSE

\begin{tabular}{|c|c|c|c|c|c|}
\hline $\begin{array}{l}\text { Table } 6 . \\
\text { factors o }\end{array}$ & $\begin{array}{l}\text { Multivari } \\
\text { ) - organi }\end{array}$ & $\begin{array}{l}e \text { analy } \\
\text { d by as }\end{array}$ & is of NUR & $\mathrm{E}$ ED (s & \\
\hline $\begin{array}{l}\text { Younger } \\
\text { speakers }\end{array}$ & Coef & Mean & $\begin{array}{c}\text { Older } \\
\text { speakers }\end{array}$ & Coef & Mean \\
\hline yqw & -47.417 & 131 & otw & -87.959 & 107 \\
\hline yqm & -41.540 & 176 & osm & -53.886 & 140 \\
\hline ysw & 4.607 & 207 & oqm & -25.226 & 193 \\
\hline $\mathrm{ytm}$ & 39.223 & 258 & oqw & 21.942 & 252 \\
\hline ysm & 70.329 & 306 & osw & 23.885 & 237 \\
\hline ytw & 96.041 & 331 & & & \\
\hline Range & 143.458 & & Range & 111.844 & \\
\hline Range ove & ll: 184.00 & & Sig. $p=0$ & & \\
\hline $\begin{array}{l}\text { Also retai } \\
\text { - Precedi } \\
\text { - Followi }\end{array}$ & $\begin{array}{l}\text { las signific } \\
\text { segment }(p \\
\text { segment ( }\end{array}$ & $\begin{array}{l}\text { nt in } m c \\
0.001) \\
0.001)\end{array}$ & & & \\
\hline
\end{tabular}


men, and that trans women are positioned at the masculine end of this continuum. This suggests that, at least for older speakers, NURSE is not a social marker of gender. The fact that it does seem to be one for younger speakers suggests that this is probably a relatively new indexicality, and may be tangled up with other social indexicalities.

Unfortunately, it is not possible to extract reliable F3 (third formant) information from this data, which makes it impossible to determine the extent to which these lowered productions of NURSE are also rounded (e.g., Espy-Wilson, Boyce, Jackson, Narayanan \& Alwan 2000; Johnson 2003; Harrington 2010), or indeed rhoticised. It may be that different variants are pulling NURSE in different directions for different groups of speakers, but we are not able to make these distinctions with this data.

\subsection{Some observations on FOOT and NURSE}

These two vowels are interesting to discuss together, because the fronting of FOOT should be pushing NURSE lower, but that appears not to be the case, given that it is different groups of speakers who are leading in the two changes. The movement of NURSE may be associated in some way with young masculinity, while FOOT seems (at least for younger speakers) to be a fairly straightforward sound change. For older speakers, the picture is somewhat complicated by the retracted realisation by straight women, potentially as a means of differentiating themselves from their queer contemporaries. This is an interesting pattern, as we have not encountered evidence of this kind of social distancing between these two groups of women very often in this data ${ }^{1}$. Other studies of vowel systems - particularly Labov, Ash \& Boberg's (2006) Atlas of North American English - have shown that isolated instances of differentiation in otherwise comparable vowel spaces can be exploited to encode social meaning. In the Atlas, differences between neighbouring dialects (regions separated by an isogloss boundary) can be salient to speakers, even if they are only defined by one or two key differences in vowel realisation. Given the social stigma of lesbian identities in New Zealand prior to the mid-1980s, it is not unreasonable to assume that there were at least some social signifiers of sexuality that women used to align or dis-align themselves with the lesbian community; it is also not unreasonable to think that at least some of these signifiers may have been linguistic. FOOT might be a good candidate for just this kind of socio-symbolic indexicality, because the relatively low phonemic density in that part of the vowel space means that there would be space for considerable articulatory variation without adversely affecting comprehensibility.

The values observed for trans speakers suggest that both vowels seem to index gendered identity to some degree, although for NURSE this seems restricted to the younger speakers. The $[+$ masculine $]$ associations of a lowered and retracted NURSE are

\footnotetext{
${ }^{1}$ DRESS showed a similar trend, but we were unable to make a strong liminality argument for the gendered indexicality of that DRESS, unlike the unambiguous pattern observed with FOOT.
} 


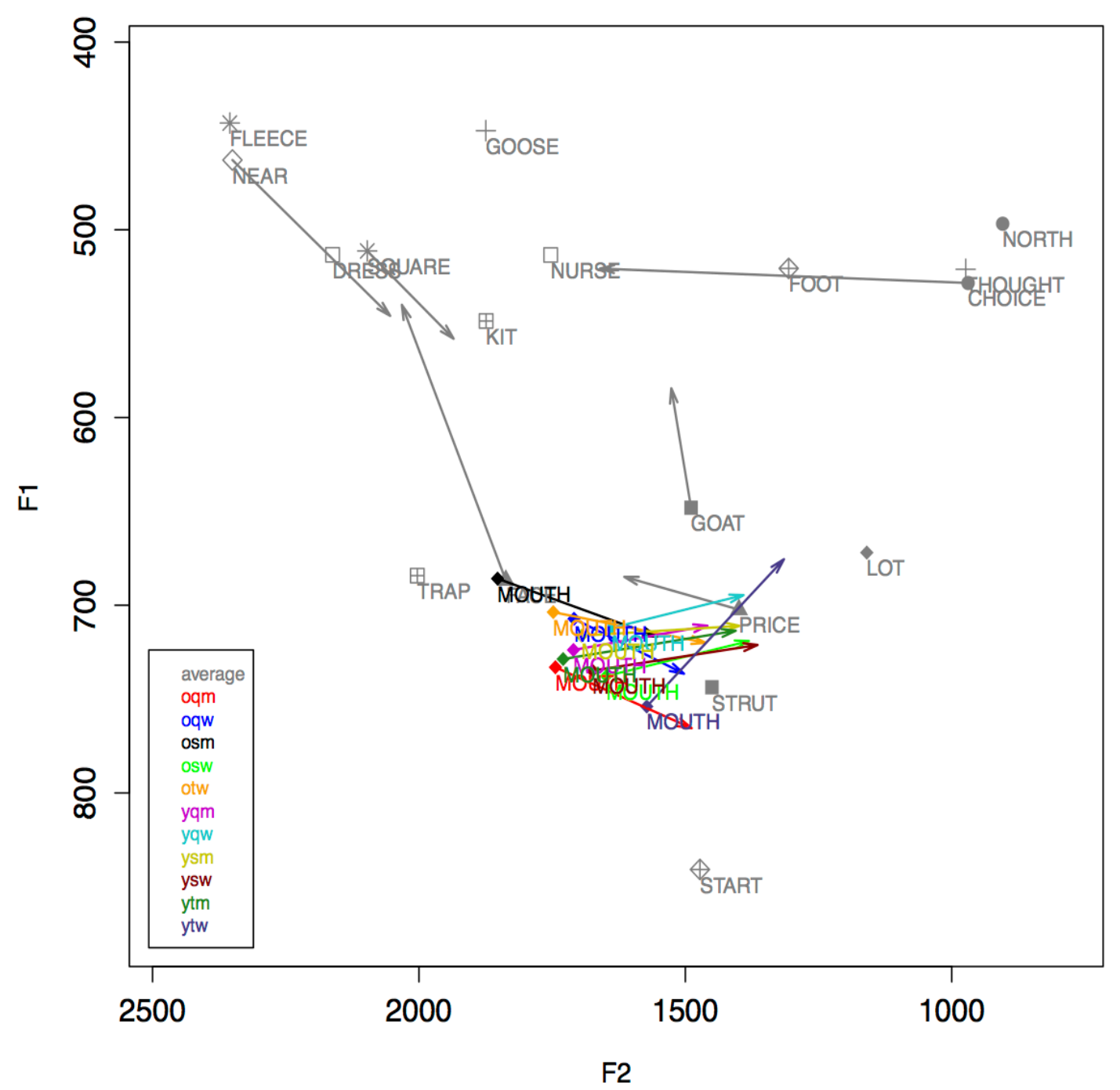

Figure 6.18: Vowel plot for MOUTH

Table 6.9: Multivariate analysis of MOUTH onset ED

\begin{tabular}{|c|c|c|c|}
\hline Gender & Coef & $\mathrm{N}$ & Mean \\
\hline women & -19.128 & 837 & 280 \\
\hline men & 19.128 & 870 & 334 \\
\hline Range & 38.256 & Sig. & $p=0.049$ \\
\hline \multicolumn{4}{|c|}{$\begin{array}{l}\text { Also retained as significant in model: } \\
\text { - Preceding segment }(p<0.001) \\
\text {-Following segment }(p<0.001)\end{array}$} \\
\hline
\end{tabular}


clearly a recent innovation, given that it is not evidenced in the patterns of the older speakers.

\subsubsection{MOUTH and PRICE}

MOUTH and PRICE are generally classed as closing diphthongs, although this classification is not particularly compelling given this data. Although some speakers do produce offglides that rise relative to their onsets, this is not universally true. The movement is perhaps more accurately described broadly front-and-back for both vowels, with some degree of height change for some speakers.

Both vowels will be discussed with respect to their onsets, as well as the general trajectories of their offglides.

\subsection{MOUTH onset}

The onset for the MOUTH diphthong is analysed using Euclidean Distance (ED), with START as a stable reference vowel. A smaller ED means the vowel is closer to START, or lower and more retracted. A larger ED means it is farther from START, and higher and fronter. Figure 6.18 shows the plot for MOUTH, and Table 6.9 gives the social component of the multivariate analysis of the onset.

The best model of the observed variation returned very little by way of social factors, meaning that the bulk of the observed variation is due to the linguistic constraints of the preceding and following segments. Gender alone was retained as significant, and neither the small range nor the $\mathrm{p}$-value close to 0.05 indicates a particularly strong effect overall. Generally, women have a slightly lower onset than men, but looking at the vowel plot in Figure 6.18, it is very possible that this is more an artefact of the behaviour of the older straight men than it is of anything more general. Older straight men have a MOUTH onset that is clearly separate from the main cluster, being higher and more fronted than anyone else. The group with the lowest and most retracted production is younger trans women, who also have the most sharply closing offglide.

\subsection{MOUTH offglide}

The offglide for MOUTH is discussed relative to its onset. A cursory examination of the plot (Figure 6.1.8) reveals that all speakers have an offglide that is back relative to its onset, but the height difference seems to depend on the starting point. Some speaker groups have an offglide that is generally closing relative to its onset (i.e., higher), and some generally opening (i.e., the offglide is lower than the onset). This distribution is summarised in Table 6.10.

Generally, the differences seem to be age-related, with younger speakers having a closing diphthong and the majority of older speakers having an opening one. Older straight women are the only exception to this, but if we consider the general Labovian principles of language change, we would expect younger speakers generally to be leading the change, and women to be at the forefront of change in their age groups. 


\begin{tabular}{cc}
\hline Table 6.10: & MOUTH offglides \\
\hline $\begin{array}{c}\text { generally } \\
\text { closing }\end{array}$ & $\begin{array}{c}\text { generally } \\
\text { opening }\end{array}$ \\
\hline yqw & osm \\
yqm & otw \\
ysm & oqw \\
osw & oqm \\
ysw & \\
ytw & \\
\hline
\end{tabular}

However, this is analysis is complicated by the fact that MOUTH is generally categorised as a closing diphthong, not an opening one. If this is an age-graded change, we would expect to find younger speakers producing the innovative variant, not the more conservative one. It may be that MOUTH is in the process of monophthongising, and we are seeing variation in the precise realisation of this process. A likely source for this confounding pattern is that most phonetic studies of New Zealand English have made use of very careful styles of speech: word lists, word pairs, and read or elicited speech. By contrast, this study is using free conversation, which is a much less guarded speech style. As a consequence, the patterns of these previous studies may not have been representative of more casual speech at the time, and so may not in fact be directly comparable to these findings.

\subsection{PRICE onset}

The onset for the PRICE diphthong is analysed along the F1 dimension, because although there is some front-back variation, the majority of the spread is in vowel height. A higher F1 value corresponds to a lower articulation, and a lower F1 value to a raised articulation. Figure 6.19 provides the vowel plot for PRICE, and Table 6.11 shows the social factors of the multivariate analysis of the onset.

The first thing we note with is that the bulk of the variation for the PRICE onset is restricted to younger speakers, who span a much larger range of coefficients than the older speakers do. Younger trans women have the lowest realisation of the onset, substantially lower than anyone else, but as previously noted, their ethnicity may be orienting them to a different norm. Setting these trans women aside, we find that the younger cis women are virtually identical in their production, but younger cis men are creating some distance between themselves.

Among older speakers, women have a generally higher articulation than men, but the difference in both means and coefficients is negligible, so it is unlikely that this is actually a meaningful difference. It seems that the onset for PRICE is relatively stable among older speakers, and that it is only recently developing into a more complex pattern. Younger trans men are patterning in line with younger queer men, suggesting that part of this complexity may be that PRICE is beginning to index gendered identity in the speech 


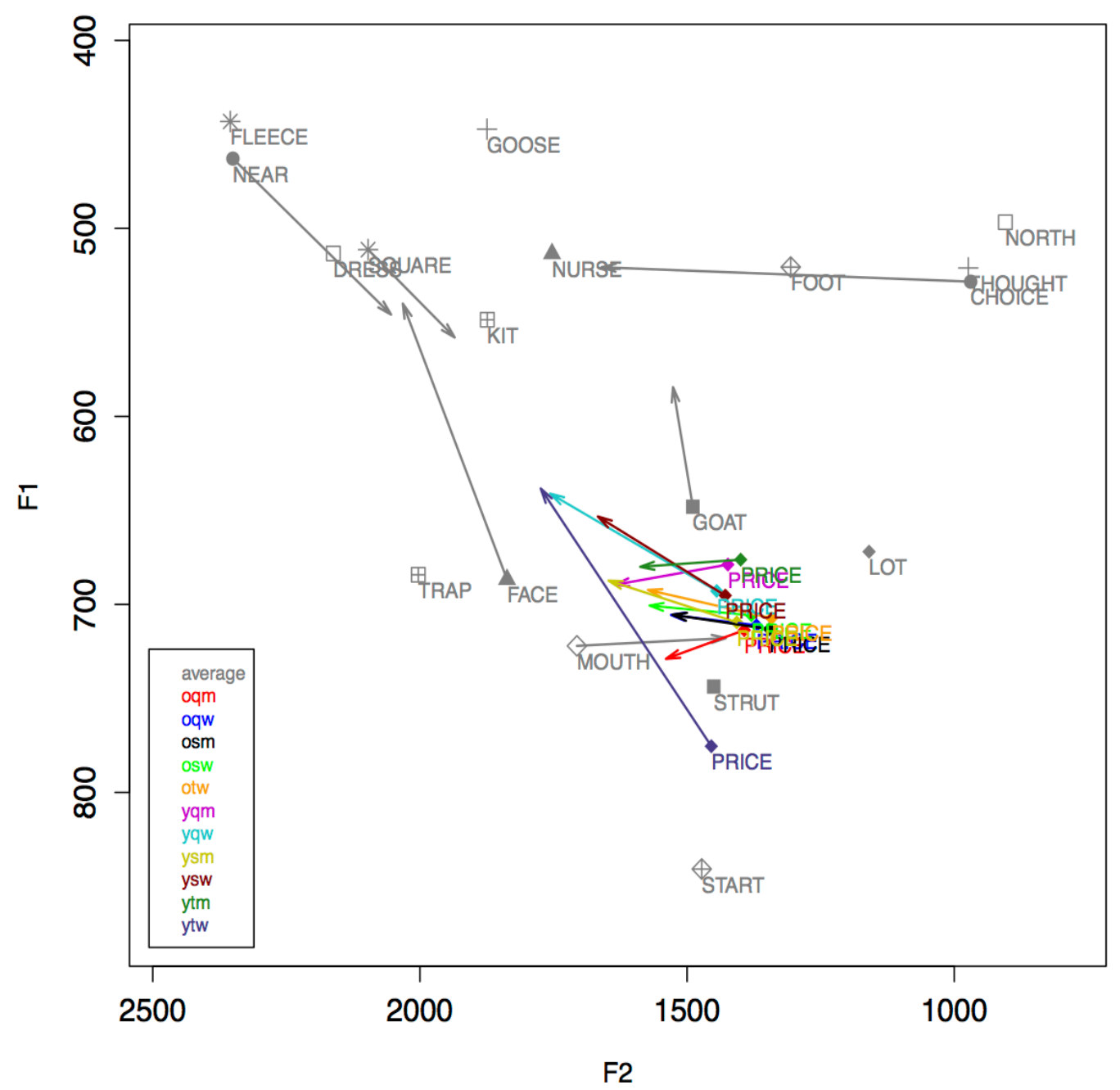

Figure 6.19: Vowel plot for PRICE

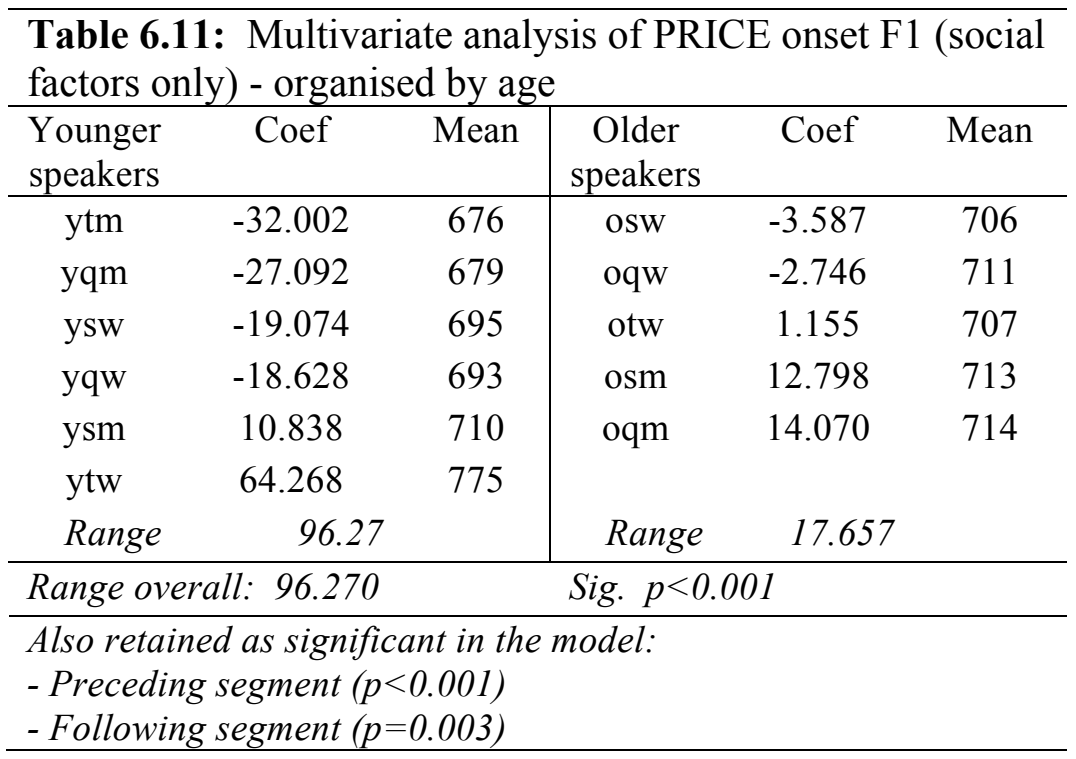




\begin{tabular}{|c|c|c|}
\hline \multicolumn{3}{|c|}{ Table 6.12: PRICE offglides } \\
\hline sharply & slightly & slightly \\
\hline closing & closing & opening \\
\hline ytw & otw & $y \mathrm{tm}$ \\
\hline ysw & osW & yqm \\
\hline yqw & ysm & oqm \\
\hline & osm & \\
\hline & oqw & \\
\hline
\end{tabular}

community, and that these trans men are making use of this new association to signal both their masculinity, and their queer identities.

\subsection{PRICE offglide}

The offglide for PRICE is discussed relative to its onset, rather than by some absolute measure, as this relative position is more representative of articulatory processes. The vowel plot shows some speaker groups for whom there is a sharp raise in the offglide, others for whom there is only a very slight raise, and others for whom there is a very slight lowering. These are summarised in Table 6.12.

We see that the speakers who have a sharply closing offglide (who show the most raising) are younger women, suggesting that the raising is an innovation - which contradicts previous observations of New Zealand English. It could be that here, as well, we see evidence of monophthongisation in progress, although it is difficult to see why young women should be the least monophthongised, if PRICE is undergoing change. However, as noted above, the bulk of previous studies of NZE has either drawn on read passages or word lists, or been conducted with speakers from other regions than Auckland. In that regard, this data may not be as directly comparable with these previous studies as we might expect. Those speakers whose offglides are slightly more open than their onsets (lowered offglides) are queer and trans men. Given that younger queer and trans men patterned similarly with respect to the onset, this similarity in treatment of the offglide is not particularly surprising. The offglide for PRICE, then, may be indexing young femininity as well as queer masculinity, by deviating from a relatively unmarked slightly-closing (or monophthongal) articulation.

\subsection{Some observations on MOUTH and PRICE}

For the onsets of both MOUTH and PRICE, we have seen that the systems are fairly stable, and the relative instability of PRICE is confined to younger speakers. The range of coefficients for both vowels is smaller than the ranges we have seen with several other vowels, so social factors do not seem to exert a particularly strong effect here. The offglides for both vowels seem in some ways to index social identity more productively, with MOUTH showing a fairly consistent effect of age, and PRICE indexing particular styles of femininity and masculinity. 


\subsubsection{STRUT and TRAP}

STRUT and TRAP are both mid-to-low monophthongs that show variation in height. In previous research (e.g., Warren 2006a), STRUT has been reported to differ from START principally in duration, but it is clear in this data that there is a qualitative distinction as well, with STRUT considerably higher than START.

In NZE, TRAP has been reported to be more raised in women (e.g., Maclagan, Gordon \& Lewis 1999; Gordon et al. 2004), but that does not seem to be markedly the case here.

\subsection{STRUT}

Although there are some differences in frontness (F2) with STRUT across the speaker groups, these differences are overshadowed by differences in height (F1), so F1 is the dimension along which this vowel is analysed. A lower F1 value represents a higher articulation than a lower one. Figure 6.20 shows the plot for STRUT, and the social constraints of the multivariate analysis re summarised in Table 6.13.

There is a general age split in the data, with younger speakers overall having a higher STRUT than older speakers, although there is some overlap towards the middle of the phonetic space. For younger speakers, there are two clusters of speakers: queer and trans men have a raised STRUT, while the coefficients for the other groups show very little effect of either raising or lowering the production. For younger speakers, then, it seems that a raised STRUT may be associated with queer masculinity, and trans men are attuned to this, even though the magnitude of the effect is not enormous.

For older speakers, there are likewise two groups: a lower STRUT being produced by older straight men and women, and a relatively neutral articulation by the other speakers groups. The trans women in this group are patterning more like the queer speakers, and closer to queer women than to queer men, suggesting that there is some degree of gendered identity salience for this cohort of speakers as well, or at least a way of indexing non-heteronormativity. This raises the possibility that some of the previous findings of a lower STRUT may be partly due to an under-sampling of queer participants.

\subsection{TRAP}

The variation that we observe in TRAP is in both frontness and height, and so is more meaningfully considered using Euclidean Distance (ED), with START as a reference vowel. A smaller ED means that the vowel is closer to START, or lower and more retracted. A larger ED therefore means that the vowel is higher and more fronted. Figure 6.21 shows the plot for TRAP, and the multivariate analysis is summarised in Table 6.14.

We can see that, generally speaking, older speakers have a higher TRAP than younger ones, with a nearly categorical distinction drawn between the two age groups, the two exceptions being older queer women and younger trans men. For younger speakers, there are arguably four groups: trans women, who have the lowest TRAP overall by a 


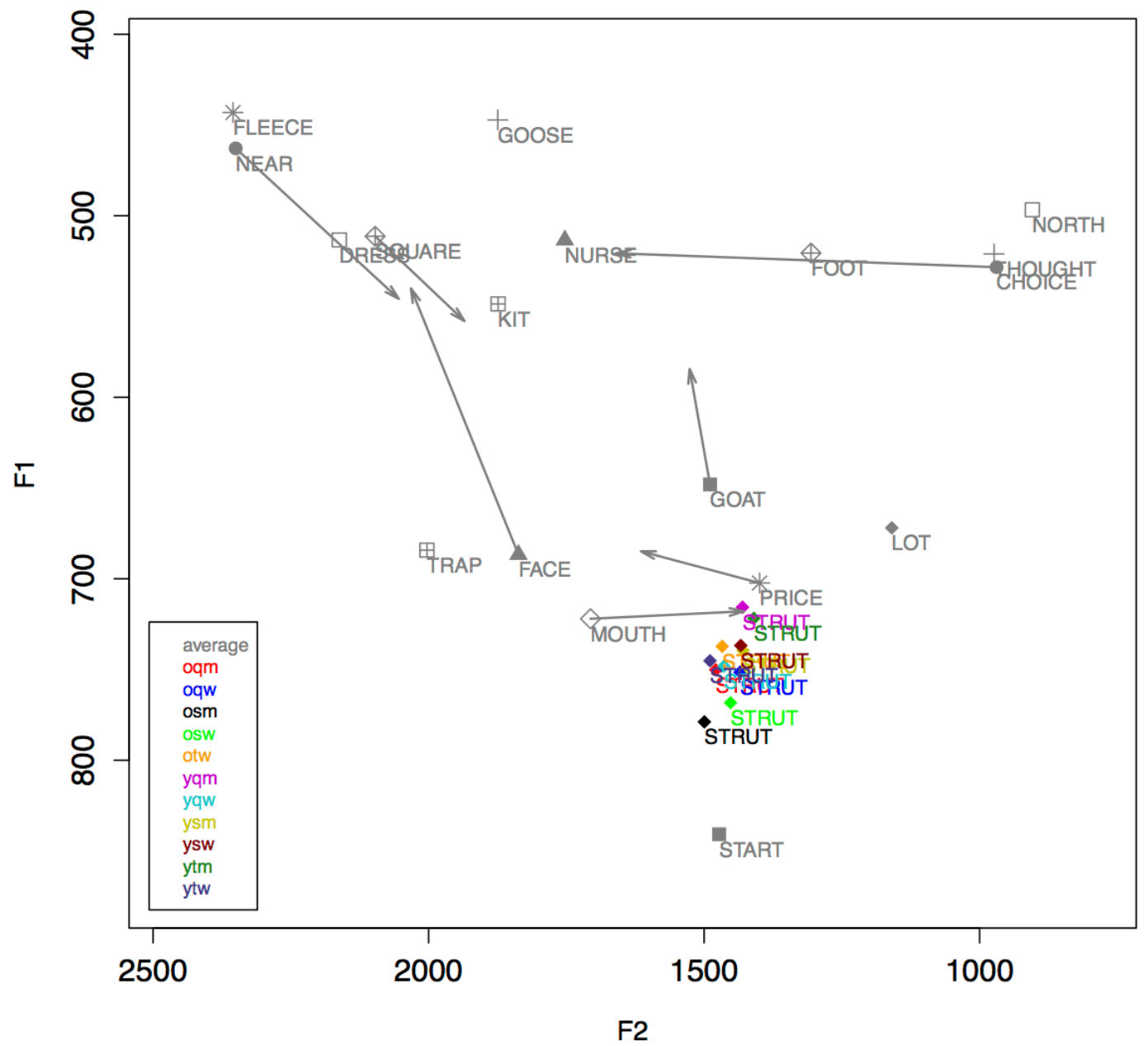

Figure 6.20: Vowel plot for STRUT

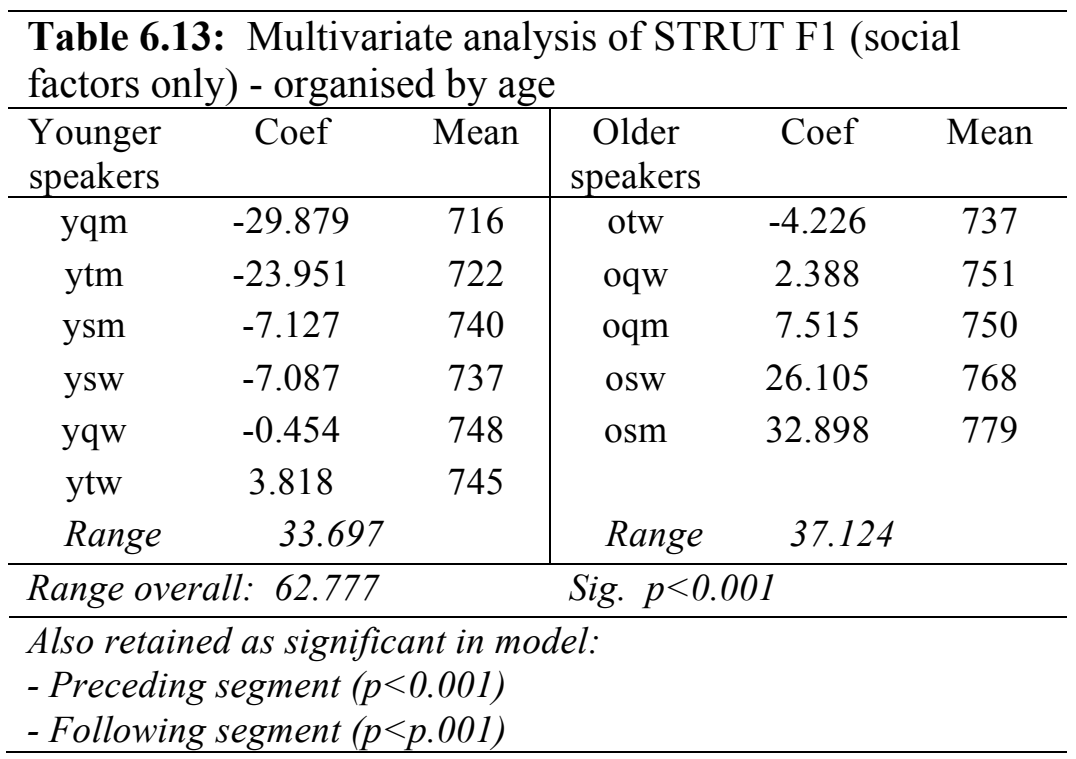




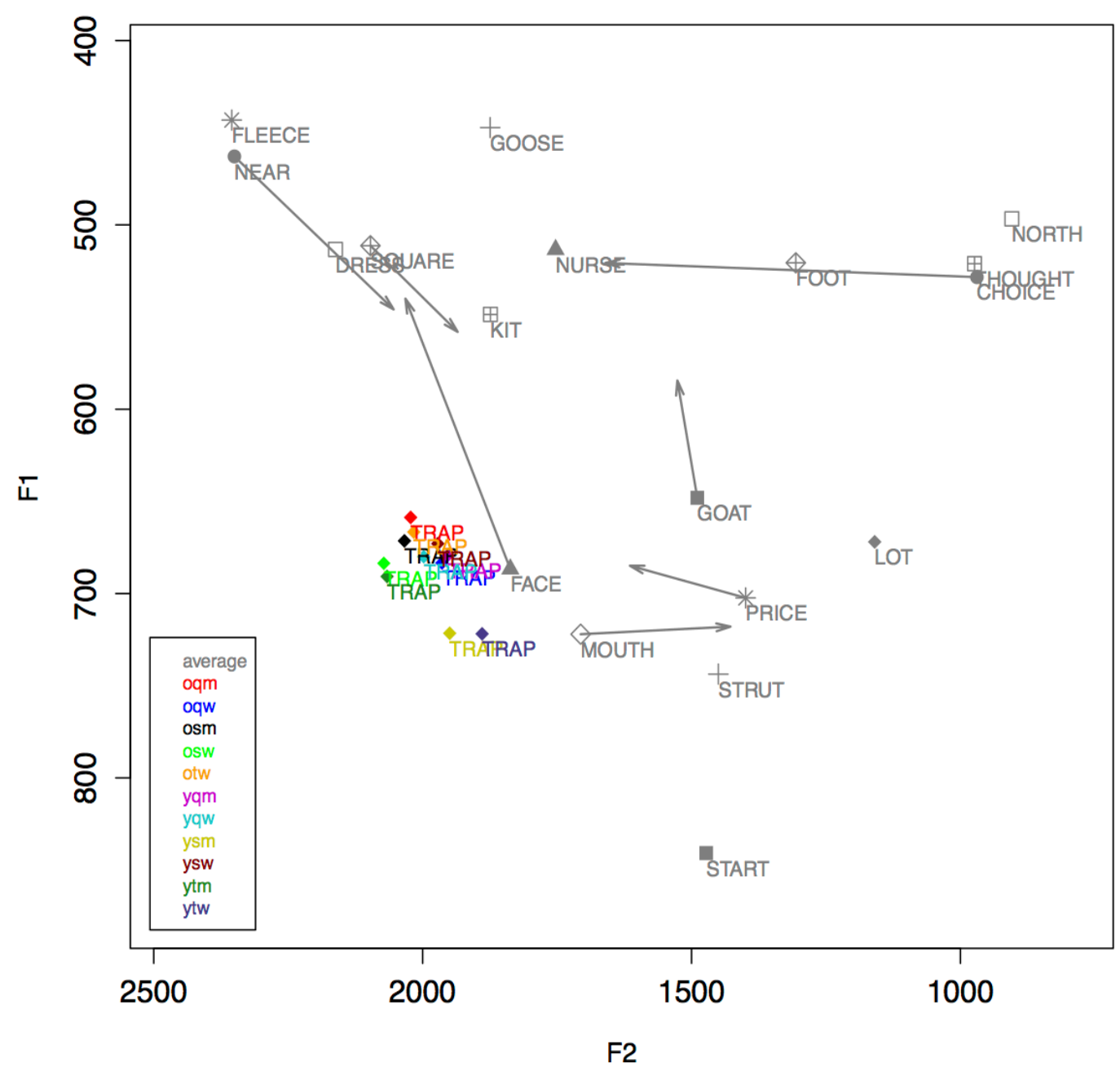

Figure 6.21: Vowel plot for TRAP

Table 6.14: Multivariate analysis of TRAP ED (social factors only) - organised by age

\begin{tabular}{lcc|ccc}
\hline $\begin{array}{l}\text { Younger } \\
\text { speakers }\end{array}$ & Coef & Mean & $\begin{array}{c}\text { Older } \\
\text { speakers }\end{array}$ & Coef & Mean \\
\hline ytm & 53.279 & 617 & osw & 73.971 & 627 \\
yqw & 2.175 & 558 & oqm & 47.819 & 586 \\
ysw & -8.369 & 536 & osm & 29.205 & 591 \\
yqm & -44.269 & 514 & otw & 17.472 & 577 \\
ysm & -50.864 & 499 & oqw & -25.559 & 530 \\
ytw & -94.861 & 441 & & & \\
Range & 148.14 & \multicolumn{5}{|c}{ Range } & 99.530 \\
\hline Range overall: 168.832 \\
\hline \multicolumn{5}{l}{ Also retained as significant in model: $p=0.015$} \\
- Preceding segment $(p<0.001)$ \\
- Following segment $(p<0.001)$ \\
\hline
\end{tabular}


considerable margin; next we find straight and queer men; then straight and queer women; and finally, trans men, with the highest. If we set aside the trans speakers for a moment, we have a fairly tidy split along gender lines, with men having a lower TRAP than women, irrespective of queer or straight identities. If we re-consider the productions of our trans participants, it looks as though TRAP does not signal gendered identity. As noted elsewhere (see $\$ 2.3 .2 .3$ ), we have reason to believe that the younger trans women may be orienting to the norms of a different variety of English, so their extremely lowered TRAP may not be directly compatible with those of the rest of the speakers.

However, there is the possibility that the variety of English that these young women are speaking - a Pasifika variety associated with South Auckland, which is locally seen as a tough neighbourhood - is indirectly influencing the productions of younger cis men; this is discussed in greater detail in section §7.4.4. The high ED for younger trans men is a bit more puzzling, in that it is not in line with either their gendered identity or with the gender they were socialised into as children. We might want to argue that they are producing a variant in line with older queer men, although there is no particularly compelling social reason to believe that older queer men would exert much influence on younger trans men. There may be other factors that were not taken into consideration in the model, which was kept linguistically simple in order to make the large number of gendered identity categories analysable within Rbrul.

For the older speakers, the distribution is more gradient, with queer women producing the lowest and most retracted variants and straight women producing the highest and most fronted. Queer men and straight men have relatively similar values, suggesting that TRAP is another locus for differentiating femininities rather than masculinities (see the discussion of FOOT in $§ 6.3 .4 .3$ for another such vowel). The trans women in this age group are patterning more closely with straight men than with queer women, which suggests that for older speakers, TRAP is not indexing gender. However, this runs counter to our argument that women are using it strategically; a different interpretation might be that, given the disparity between queer and straight women, trans women are aiming for a middle ground that doesn't particularly signal either a queer or a straight identity.

\subsection{Some observations on STRUT and TRAP}

Both STRUT and TRAP seem to index younger masculinity, albeit in different directions: with TRAP, younger (cis) men produce generally lower and more retracted variants, while with STRUT, younger (cis and trans) men have a higher articulation; in other words, younger men seem to be neutralising height differences between these two vowels. The speech norms of Pasifika Auckland English (or arguably South Auckland English more generally) may be affecting this height levelling - a possible indexical chain for this process is discussed in $§ 7.4 .4$.

This pattern is not the same one that we observed among older speakers, where the more relevant social dimension seems to be sexuality. This is especially true for 
women, who show a particularly marked queer-straight distinction with TRAP. Although speech differences between queer and straight speakers are not as stereotypically salient for women as they are for men, this does not mean that there are none, and this data suggests that with at least a small handful of vowels (which are often below the level of conscious awareness, and tend not be explicitly talked about), women are able to signal their identities in some way, however subtly and under-the-radar those signals may be.

\subsubsection{Mergers, near-mergers, and unmergers}

Vowel mergers are a fairly common process of language change, but is this process reversible? Intuitively, it seems unlikely - if a distinction between two sounds is truly lost, then so too must be the information necessary to separate the sounds again. And yet historical linguists can point to apparent unmergers over the course of a language's development, suggesting that the trajectory is not entirely unidirectional. Jespersen (1949) discusses the role that spelling may have had in re-establishing previously merged word classes in English, while Nunberg (1980) draws attention to the role of allophonic variation in the processes of merging and unmerging. Indeed, the phonetic environment is clearly implicated in a number of mergers (e.g., Hickey 2014), drawing into question whether some sound changes truly are mergers or not.

Labov (1994) argued against the idea of unmergers on principle, asserting that "once a merger, always a merger" (p.311). Rather, he claimed that when an apparent unmerger was taking place, the phonemes in question were a near-merger rather than a full merger. The critical difference between a merger and a near-merger is that in a nearmerger, not all members in a community participate equally in the merger (Labov, Yaeger $\&$ Steiner 1972), whereas with a full merger, the distinction is completely neutralised and, presumably, unrecoverable. With not all speakers merging the two sounds, and with the sound change phonologically patterned, it becomes much easier to see how two sounds popularly thought to be merged can be disentangled again.

New Zealand English has three vowel pairs that have been variably reported as merged, nearly merged, or in the process of merging: the diphthongs NEAR and SQUARE, the monophthongs START and STRUT, and the monophthongs THOUGHT and NORTH/FORCE. Each pair is discussed below.

\subsubsection{NEAR and SQUARE}

It is all but impossible to present any kind of discussion of vowels in New Zealand English without addressing the status of NEAR and SQUARE. Because NZE is a generally non-rhotic variety of English, NEAR and SQUARE are realized as diphthongs, and highly variable ones at that. Watson, Maclagan \& Harrington (2000) note that the onsets for the two diphthongs were distinct in early NZE, but have since converged in the last 60 or 70 years. Although there has been some debate about the direction of the merger (e.g., Holmes \& Bell 1992; Batterham 1995; Watson, Maclagan \& Harrington 


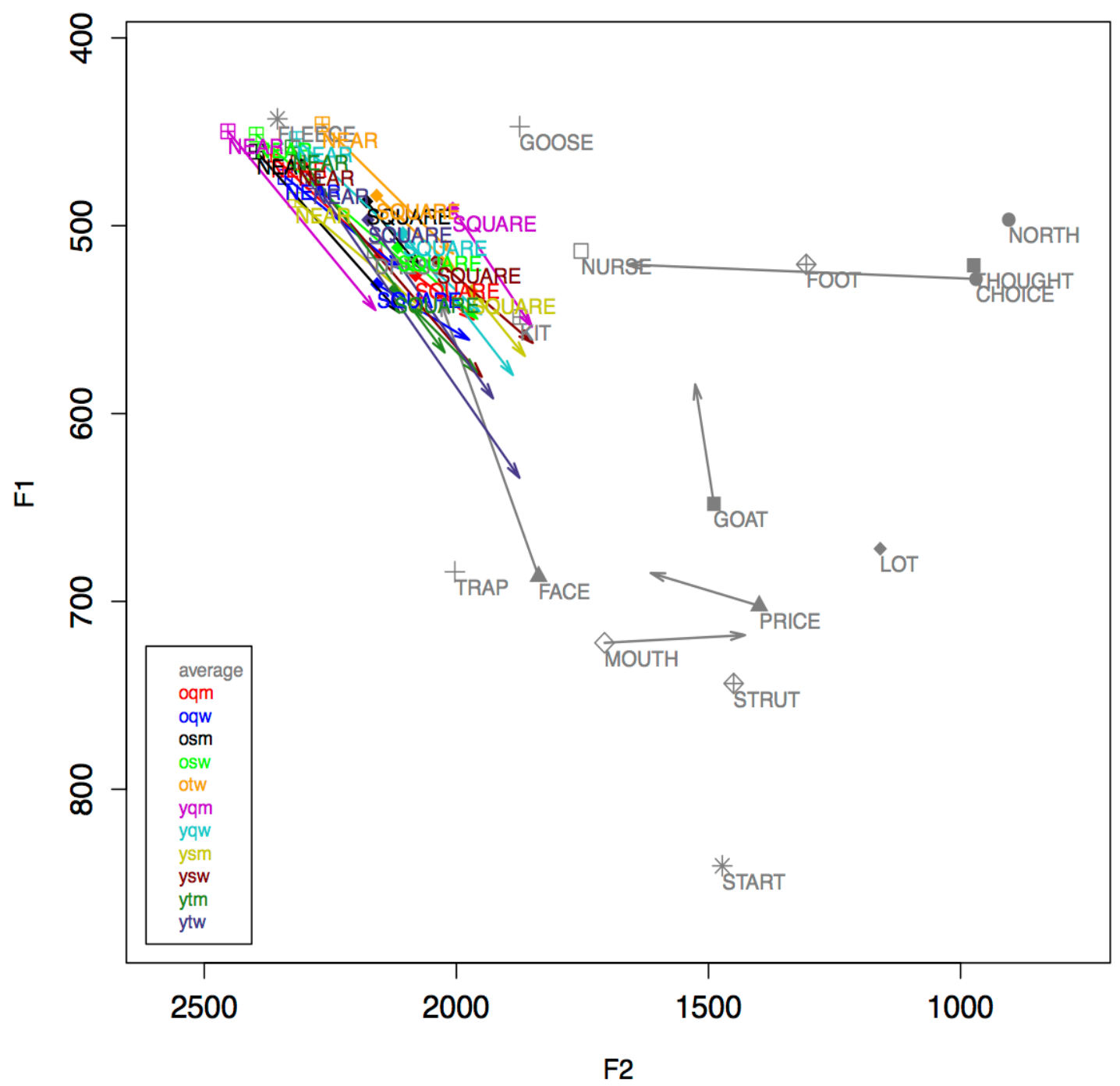

Figure 6.22: Vowel plot for NEAR and SQUARE

Table 6.15: (Un)merger of NEAR and SQUARE organised by age

\begin{tabular}{cccc}
\hline younger & $E D$ & older & $E D$ \\
\hline ytw & 111 & otw & 114 \\
ytm & 216 & oqw & 191 \\
yqw & 217 & osm & 220 \\
ysw & 280 & osw & 287 \\
ysm & 354 & oqm & 293 \\
yqm & 448 & & \\
\hline
\end{tabular}


2000), the majority opinion seems to be that it has moved in the direction of NEAR rather than SQUARE (Gordon \& Maclagan 2001; Warren, Rae \& Hay 2003), although it is not clear that this is universally true across the different regional dialects of NZE.

The merger has been shown to be driven by age and gender (Holmes \& Bell 1992; Maclagan \& Gordon 1996; Watson, Harrington \& Evans 1998; Gordon \& Maclagan 2001), with younger women leading the change. It has also been shown to be influenced by socioeconomic status (Bauer 1986; Batterham 1995; Maclagan \& Gordon 1996; Gordon \& Maclagan 2001), with speakers of lower socioeconomic background more merged than those with higher status. Perceptually, Warren, Rae \& Hay (2003) found that NEAR could be interpreted as either NEAR or SQUARE, but SQUARE was only ever interpreted as SQUARE, while Bauer \& Warren (2004b) noted that younger speakers often couldn't distinguish between NEAR and SQUARE, in either production or perception.

Warren (2004), somewhat unexpectedly, reported that women were beginning to have a less merged NEAR and SQUARE than men, suggesting that the 'merger' was possibly reversing. This was further supported by Hay, Drager \& Warren (2009), who found that the most merged word pairs were produced by people in their 30s, while both younger and older speakers produced less merged pairs. Warren (2005) also noted that the realization of SQUARE was being influenced by the tongue position of the previous segment. He reported higher SQUARE variants in the context of a preceding $/ \mathrm{J} /, \mathrm{t} \mathrm{f} /$, or $/ \mathrm{k} /$, all of which he classified as [+coronal]. In the case of NEAR and SQUARE in New Zealand English, then, it is clear that there never was a full merger, but rather a nearmerger (Labov 1994). There have always been social variables associated with the convergence, meaning that by definition not all speakers were participating equally; additionally, there are phonological factors influencing the gradient convergence of the two sounds. This means that the phonetic information needed to tell the two vowels apart was retained at the level of the community, even if some groups within the population had merged them, and therefore it is possible to re-split the near-merged pair into their original sets.

\subsection{NEAR and SQUARE in this data}

Given that NEAR and SQUARE have recently approached merger in NZE, and have even more recently started moving away from each other, we might expect to find both age and gender patterns in our data. Figure 6.22 provides a vowel plot for NEAR and SQUARE, showing both onsets and glides for all speakers.

The first thing we notice is that the onsets do not seem to be merged generally. NEAR starts in the vicinity of FLEECE, and SQUARE in the vicinity of DRESS. The two clusters of onsets do not overlap, although a close examination shows that some groups of speakers have onsets that are closer than others. We can also see that, for some groups, the larger separation is along the F1 dimension, while for others it is along the F2 dimension. 
Because these two vowels have been variable for about as long as they have been studied in NZE, the precise F1 and F2 values are perhaps less telling than the relative positions of each vowel for each group of speakers - that is to say, how closely merged or unmerged the onsets are. The easiest way to do this is to consider the Euclidean Distance (ED) between each pair for each group of speakers; Table 6.15 provides the calculated ED between NEAR and SQUARE across all speaker groups (the means across gender groups are provided in Appendix D). A smaller ED means the two onsets are closer together. Note that a more sophisticated analysis of this pair of vowels is complicated by unequal numbers, so we will restrict ourselves to a descriptive examination only.

For younger speakers, the least merged groups are the queer and straight men, and the most merged group is trans women. More generally, there seems to be a gender split among younger speakers, with trans men having a degree of merger that is more comparable to cis women than to cis men.

For older speakers, we see that straight women and queer men have about the same degree of onset merger, as do straight men and queer women. This pattern is suggestive of stereotypes of gay men speaking like women, and lesbians speaking like men. It is interesting that this pattern is not evident in the younger speakers, so it may be that the relevance of these stereotypes is decreasing as queer identities become more mainstreamed in Auckland. Trans women pattern most closely with queer women, at the merged end of the scale, but the difference between EDs are in fact greater between trans and queer women than between queer women and straight men, so it is not completely clear that queer femininity is either the source or the target for the trans women's production.

The onsets for NEAR and SQUARE suggest that younger men are leading the current trend towards an unmerger, but the behaviour of trans speakers makes it unclear the extent to which this is accessible as a marker of gendered identity. The offglides for both vowels are closing and generally parallel, and do not suggest a gendered pattern either.

\subsubsection{NORTH and THOUGHT}

In New Zealand English, the lexical sets of NORTH and THOUGHT are generally treated as the same set (e.g., Hay, Maclagan \& Gordon 2008) as the source dialects of English (those spoken by the early British settlers to New Zealand) had a merged NORTH/THOUGHT vowel (e.g., Gordon et al. 2004; Wells 1982). The decision, then, to investigate the two as potentially separate lexical sets was motivated by observations of inconsistencies in people's pronunciation. It wasn't clear what the differences were, only that the two seemed at times to have distinct vowels, and at others, they sounded clearly merged.

These observations, although anecdotal and limited, were enough to make it worthwhile to consider them as separate vowels for the purposes of this study, as the 


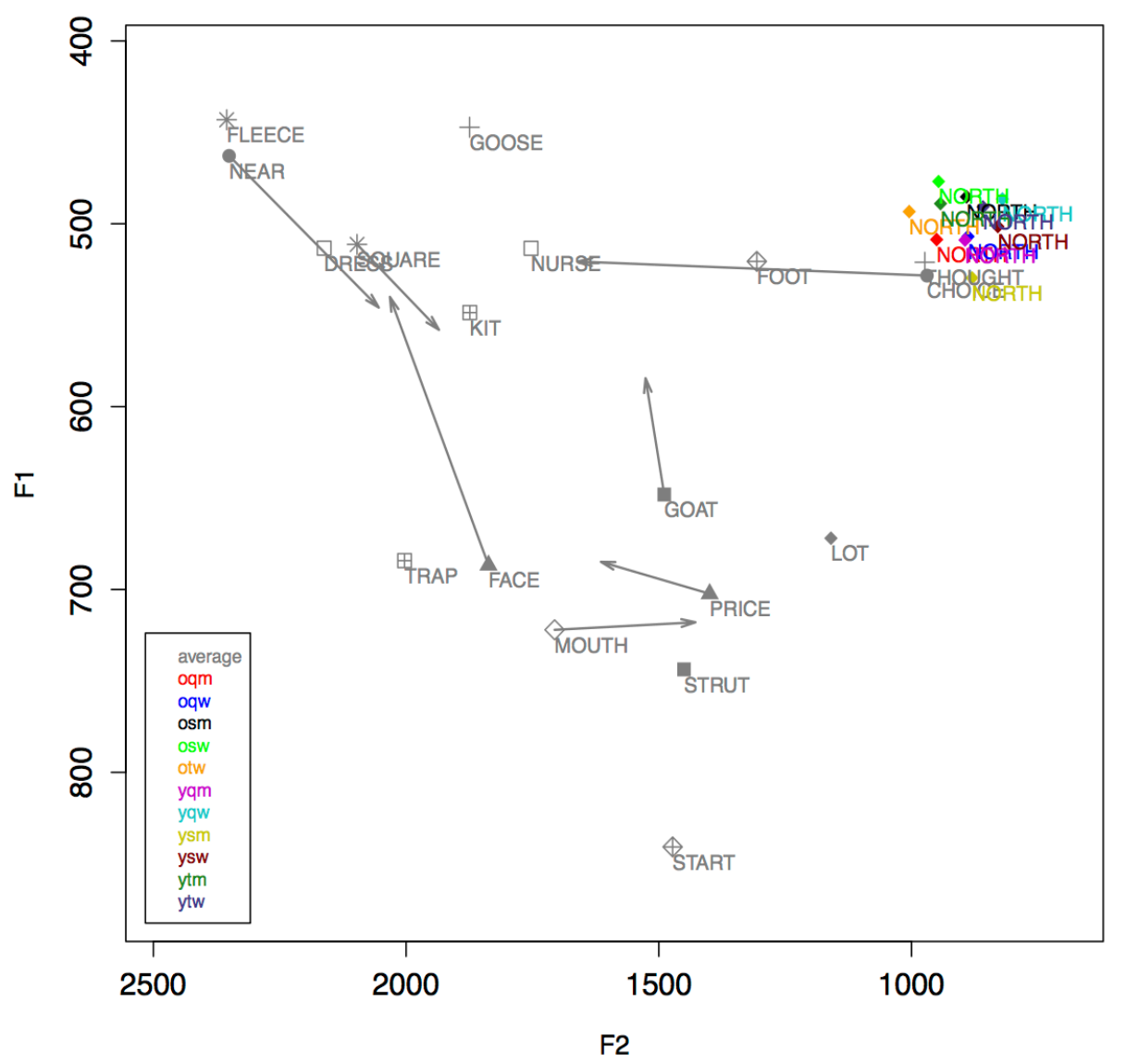

Figure 6.23: Vowel plot for NORTH

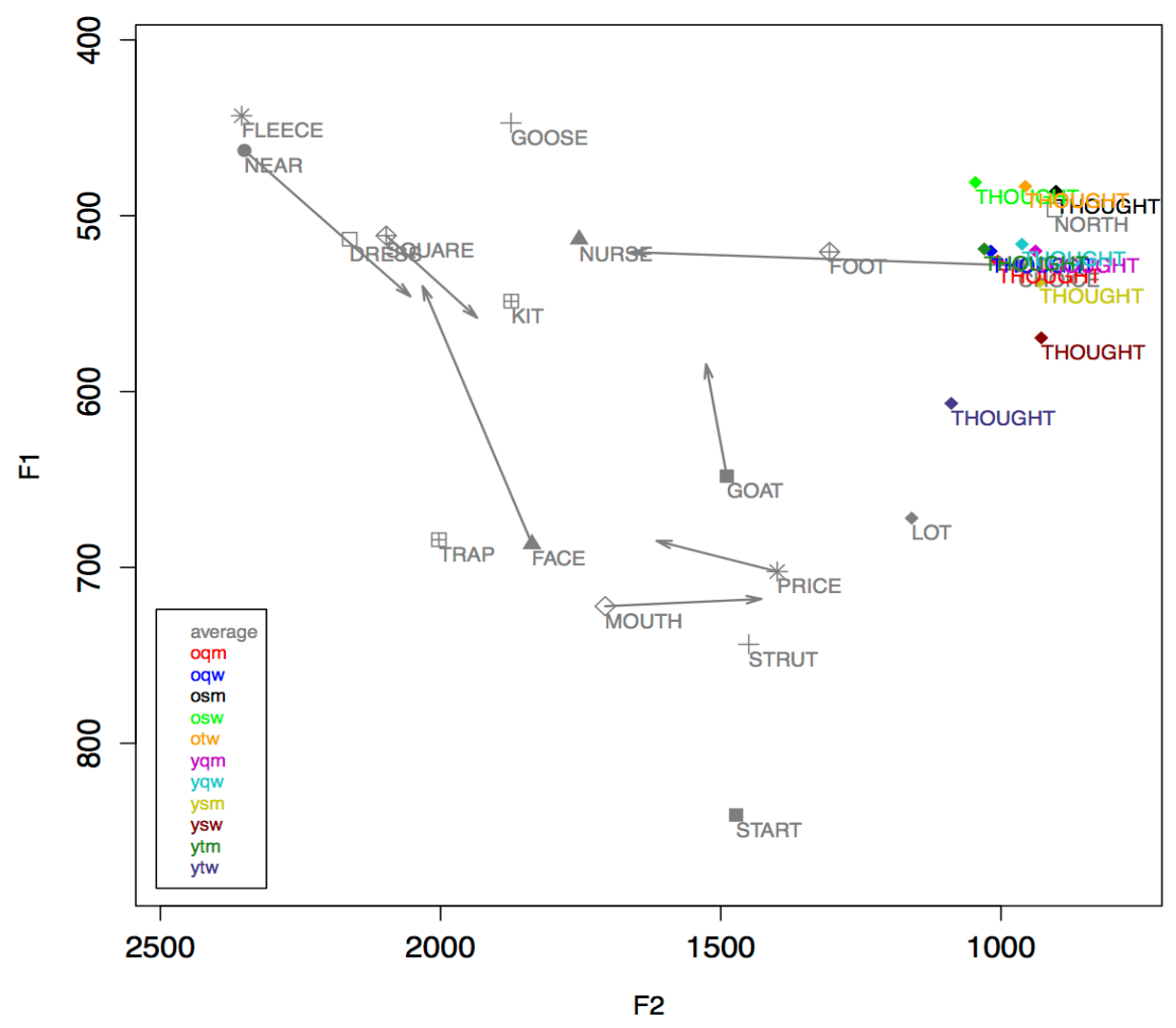

Figure 6.24: Vowel plot for THOUGHT 


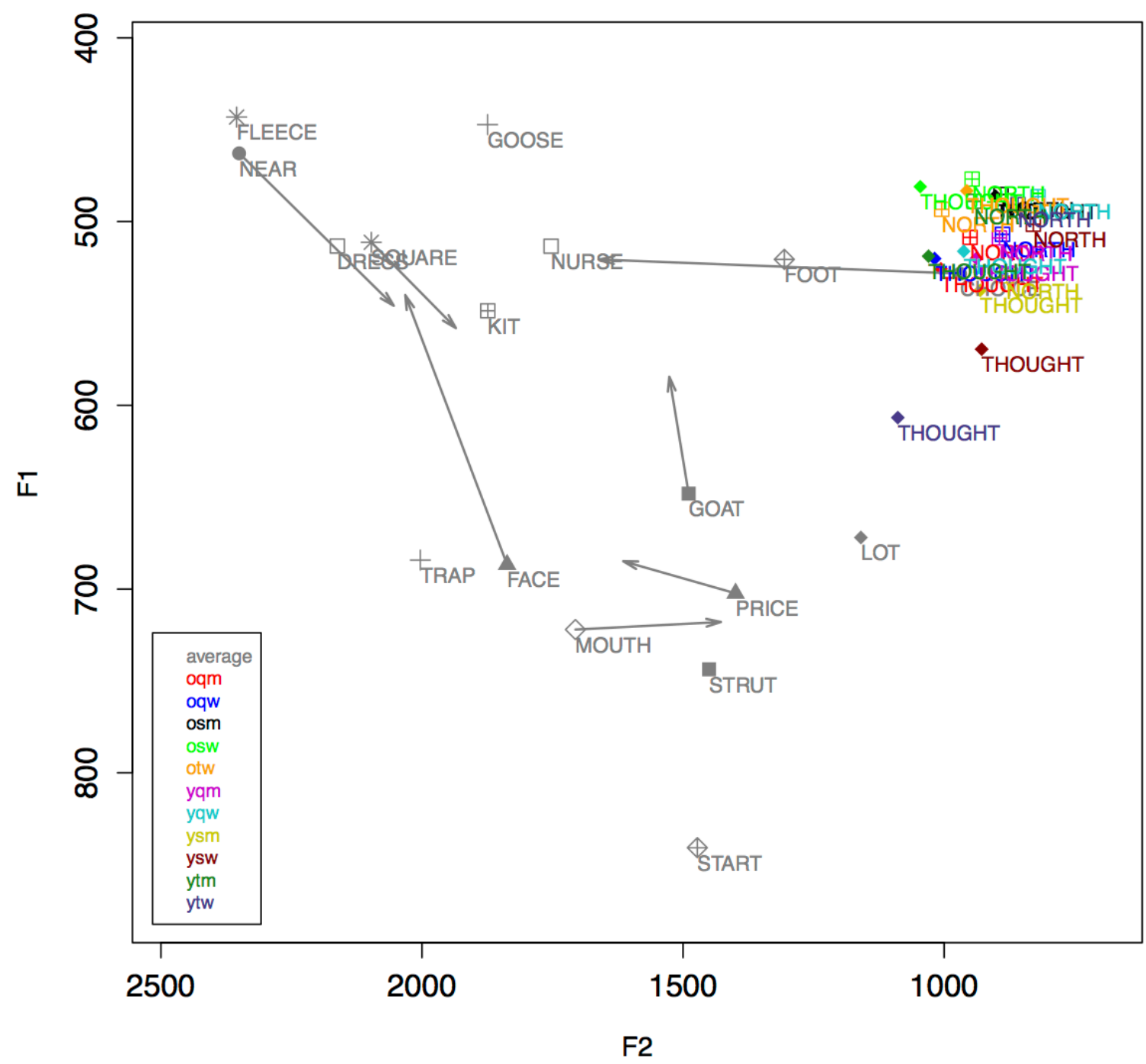

Figure 6.25: Vowel plot for NORTH and THOUGHT

Table 6.16: Separation of NORTH and THOUGHT (ED)

\begin{tabular}{clcl}
\hline older & $E D$ & younger & $E D$ \\
\hline osm & 11 & yqm & 46 \\
otw & 49 & ysm & 51 \\
oqm & 58 & ytm & 92 \\
osw & 99 & ysw & 120 \\
oqw & 131 & yqw & 145 \\
& & ytw & 258 \\
\hline
\end{tabular}


inconsistencies in these observations may have been driven by social differences that would be evident in the data.

\subsection{NORTH and THOUGHT in this data}

Figures 6.23 and 6.24 above provide the individual plots for NORTH and THOUGHT. These plots show that NORTH is fairly compactly clustered, while THOUGHT has several smaller groupings in F1. For NORTH, although there are some differences between speaker groups in this data, there is no clear trend in the data suggesting a change or dispersal along a particular track. The plot for THOUGHT shows some separation in F1. There is one cluster of older speakers (older straight women, older straight men, and older trans women) who have the highest mean F1, then another group (younger straight men, younger trans men, and all groups of queer speakers) who have a middling mean F1, and then younger trans women and younger straight women having the lowest $F 1$ values by a considerable margin.

When we consider the two vowels together (Figure 6.25), the picture changes somewhat: THOUGHT and NORTH seem largely merged for everyone except for younger trans men and straight women, whose mean F1 values for THOUGHT are considerably lower than the general cluster.

Because we are more interested in whether NORTH and THOUGHT are (un)merged than we are in the particular values of either, we will consider each group's vowel pair using a Euclidean Distance calculation to approximate the distance between the two target vowels. These values are provided in Table 6.16, separated by age group. Here, a smaller number indicates that the pair is more closely merged than those with a larger number. Because of uneven numbers of tokens with the two data sets, and the realities of accounting for two sets of phonetic environments, a more complex statistical analysis was not possible, so we will consider general distribution only.

There is a fairly consistent gender effect in this data, with women being generally less merged than men. This effect is more pronounced in the younger speakers than in the older ones, suggesting a general trend towards an unmerger, as we would expect younger speakers and women to be leading phonetic changes that are under the radar. That older trans women pattern more like men suggests that this is not a

particularly salient difference with regards to gendered identity, although the separation of THOUGHT and NORTH among younger trans men - being roughly midway between younger straight men and women - does not provide any evidence in either direction.

\subsubsection{START and STRUT}

The vowel pair of START and STRUT are said to be chiefly differentiated by length (e.g., Bauer \& Warren 2004a; Watson, Harrington \& Evans 1998; Maclagan 1982) rather than formant values. It is worth noting, however, that Watson, Maclagan \& Harrington (2000: 59) suggested that $F 2$ differences between the two vowels observed in speakers of an earlier dialect of NZE - where STRUT was considerably higher than START - were due 


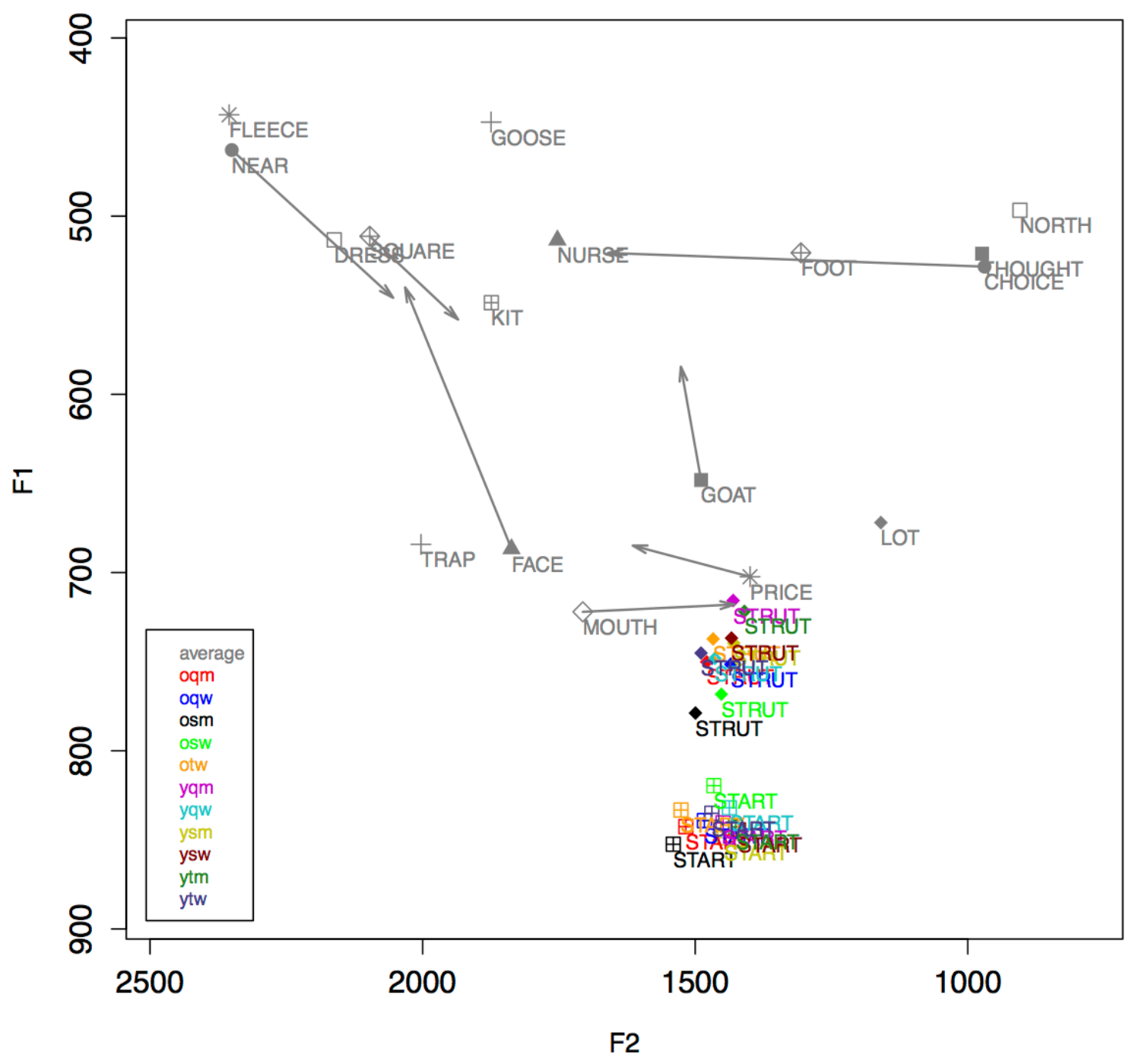

Figure 6.26: Vowel plot for START and STRUT

Table 6.17: Separation of START and STRUT (ED)

\begin{tabular}{clcl}
\hline younger & $E D$ & older & $E D$ \\
\hline yqw & 88 & osw & 53 \\
ytw & 92 & osm & 84 \\
ysw & 108 & oqm & 100 \\
ysm & 110 & oqw & 100 \\
ytm & 122 & otw & 113 \\
yqm & 126 & & \\
\hline
\end{tabular}


to the continuous speech of the recordings, as opposed to the citation and $/ \mathrm{hVd} /$ form used in later recordings. In other words, the previously observed height similarities may be more a product of careful speech style, and what New Zealanders think the vowel should sound like, than reflecting what these speakers actually do in their everyday lives. As this study is also using continuous speech, then, height differences are perhaps to be expected. Where this perceived norm is coming from is a different question, as it does not seem to align particularly with an RP production; it will make an interesting investigation for the future.

\subsection{START and STRUT in this data}

Figure 6.26 provides the vowel plots for START and STRUT on the same set of axes, as there is no overlap between the two spaces to make the plot difficult to read. We see that START is fairly tightly clustered, while STRUT shows some spread in F1. The highest mean F1 values for STRUT are produced by younger queer and younger trans men, while the lowest are those of the older straight men and women; STRUT on its own was discussed in greater detail in section $§ 6.3 .4 .5$. Because we are primarily interested in the degree to which these two vowels are merged or unmerged, we will consider each vowel pair as separated by a calculated Euclidean Distance. In Table 6.17, a lower ED value means the two vowels are closer together.

START and STRUT for younger queer men and younger trans men are the furthest from merged, and older straight women are the closest. Among younger speakers, women are less merged than men. Younger trans participants are patterning with their identified gender, suggesting that START-STRUT may signal gender interpretably for these speakers. Among older speakers, the difference seems primarily driven by sexuality: straight speakers are less merged than queer ones, and trans women are patterning more closely with their queer contemporaries.

However, it should be noted that range of EDs is generally not enormous, and at least from a production perspective, the two vowels are clearly unmerged - likely attributable to the spontaneous speech nature of the data under consideration here (cf. Watson, Maclagan \& Harrington 2000: 59). It is not clear that there is much by way of socially-meaningful difference attributable directly to the separation of START and STRUT; any differences are likely due to STRUT-raising, as discussed in $§ 6.3 .4 .5$ above.

\subsubsection{Comparing vowel spaces: the Index of Similarity}

So far, we have been looking at vowels in relative isolation: how does vowel $\mathrm{X}$ differ among our various social groups? We have seen that some vowels show quite a lot of difference, along the lines of gender or sexuality or age, or some combination of these factors. Some vowels show relatively little variability across groups of speakers.

But what about whole vowel spaces as a unit in and of themselves? If groups A and B show differences in three vowels, and groups B and C show differences in four 


\begin{tabular}{|c|c|c|c|c|c|c|c|c|c|c|}
\hline \multicolumn{11}{|c|}{ DRESS: ED } \\
\hline & oqm & oqw & osm & osw & otw & yqm & yqw & ysm & ysw & ytm \\
\hline ytw & 1 & 1 & 1 & 1 & 1 & 1 & 1 & 1 & 0 & 1 \\
\hline ytm & 0 & 0 & 1 & 1 & 1 & 1 & 0 & 0 & 0 & \\
\hline ysw & 0 & 0 & 0 & 0 & 0 & 0 & 0 & 0 & & \\
\hline ysm & 1 & 1 & 1 & 1 & 0 & 1 & 1 & & & \\
\hline yqw & 1 & 1 & 0 & 1 & 0 & 1 & & & & \\
\hline yqm & 1 & 1 & 1 & 1 & 1 & & & & & \\
\hline otw & 0 & 0 & 0 & 1 & & & & & & \\
\hline osw & 1 & 1 & 1 & & & & & & & \\
\hline osm & 0 & 0 & & & & & & & & \\
\hline oqw & 1 & & & & & & & & & \\
\hline
\end{tabular}

other vowels, can we talk about how similar the vowel spaces of groups $\mathrm{A}, \mathrm{B}$, and $\mathrm{C}$ are? To do so, we need some kind of generalisable metric to compare them: we will use an Index of Similarity (IoS), devised for this thesis as a means of comparing multiple groups ${ }^{2}$. The IoS approach is to use a "same/different" count across vowels, with pairwise comparisons between all of the groups. The following sections describe the procedure for calculating IoS, and what such an index tells us about the vowel spaces of our eleven gendered groups.

\subsubsection{Computing pairwise comparisons}

A same/different count requires some kind of means testing, and as discussed in $\$ 3.6 .1 .1$, a straightforward t-test is not appropriate for multiple comparisons. Instead, we will use ANOVA, and Tukey's HSD post-hoc tests to determine the same/difference score for each pairwise comparison. We will test each measurement that was statistically analysed in the sections $\S 6.3 .4 .1$ to $\S 6.3 .4 .5$ calculated for each token (either F1, F2, or a Euclidean Distance - group mean F1, F2 and ED values are provided in Appendix D). This approach yielded ten sets of pairwise comparisons which were tallied: pairs that were not significantly different were scored 1 (same), and those that were significantly different were scored 0 (different).

As an example, the counts for the Euclidean Distance (ED) measurement for DRESS are given in Table 6.18. The light grey row in this table shows that the DRESS ED for younger trans women was significantly different only from the younger straight women (score of 0 ); all others were scored a 1 , which indicates that there was no significant difference. In contrast, the darker grey shaded regions show that younger straight women are significantly different from all groups.

For the Euclidean Distance calculated for the onset of FACE, the counts are

${ }^{2}$ I am indebted to Dr Richard Arnold of the VUW School of Mathematics and Statistics for his guidance and suggestions on this approach. 


\begin{tabular}{lcccccccccc}
\hline \multicolumn{10}{l}{ Table 6.19: } & Sample Index of Similarity (Ios) counts (2) \\
\hline \multicolumn{10}{l}{ FACE onset: } & $E D$ \\
\hline oqm & oqw & osm & osw & otw & yqm & yqw & ysm & ysw & ytm \\
ytw & 0 & 0 & 0 & 0 & 0 & 0 & 0 & 0 & 0 & 0 \\
ytm & 0 & 0 & 0 & 0 & 0 & 1 & 1 & 1 & 1 & \\
ysw & 0 & 0 & 0 & 0 & 0 & 0 & 1 & 1 & & \\
ysm & 0 & 0 & 0 & 0 & 0 & 1 & 1 & & & \\
yqw & 0 & 0 & 0 & 0 & 0 & 1 & & & & \\
yqm & 0 & 0 & 0 & 0 & 0 & & & & & \\
otw & 1 & 1 & 0 & 1 & & & & & & \\
osw & 1 & 1 & 0 & & & & & & & \\
osm & 0 & 0 & & & & & & & & \\
oqw & 1 & & & & & & & & \\
\hline
\end{tabular}

presented in Table 6.19. This second table is read in exactly the same way as that given for DRESS.

We can then add the two counts tables together, to give us a cumulative same/different score for each pairwise comparison. The highlighted column in Table 6.20 shows the cumulative score for older queer men. For both DRESS and FACE, their vowels were the same as those of older straight women and older queer women (scores of 2 ); in contrast, their values were different on both counts from those of younger trans men, younger straight women, and older straight men (scores of 0 ). For the remaining groups, they were scored same on one of the vowels and different on another. What this table shows us is that, using only these two vowels as points of comparison, older queer men have a vowel space that is more similar to older straight women and older queer women than to the other groups.

At this point, of course, we are not actually interested in the particulars of DRESS or FACE: we have already explored these vowels in detail (§6.3.4.2.1 and

\begin{tabular}{|c|c|c|c|c|c|c|c|c|c|c|}
\hline \multicolumn{11}{|c|}{ DRESS ED and FACE onset ED } \\
\hline & oqm & oqw & osm & osw & otw & yqm & yqw & ysm & ysw & ytm \\
\hline ytw & 1 & 1 & 1 & 1 & 1 & 1 & 1 & 1 & 0 & 1 \\
\hline ytm & 0 & 0 & 1 & 1 & 1 & 2 & 1 & 1 & 1 & \\
\hline ysw & 0 & 0 & 0 & 0 & 0 & 0 & 1 & 1 & & \\
\hline ysm & 1 & 1 & 1 & 1 & 0 & 2 & 2 & & & \\
\hline yqw & 1 & 1 & 0 & 1 & 0 & 2 & & & & \\
\hline yqm & 1 & 1 & 1 & 1 & 1 & & & & & \\
\hline otw & 1 & 1 & 0 & 2 & & & & & & \\
\hline osw & 2 & 2 & 1 & & & & & & & \\
\hline osm & 0 & 0 & & & & & & & & \\
\hline oqw & 2 & & & & & & & & & \\
\hline
\end{tabular}




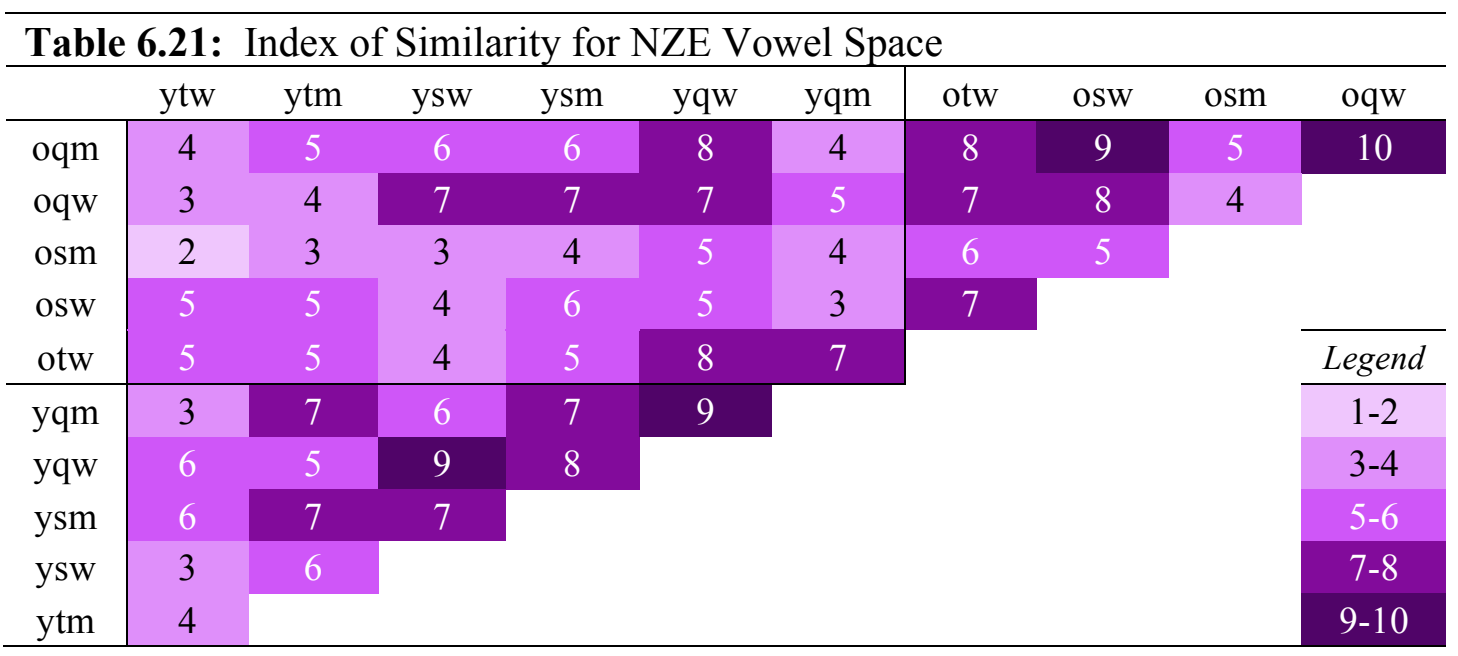

\$6.3.4.1.1, respectively). But we can go through this same/different tally process with the remaining 8 measurements, and arrive at a grand total for each pairwise comparison of gendered identities: the Index of Similarity.

\subsubsection{Results}

Table 6.21 below provides the summed totals for the Index of Similarity across all measurements of all vowels. The maximum total possible is 10, indicating a $100 \%$ overlap of the vowel space, at least with regards to the vowels analysed here.

Pairings with higher IoS scores show that those groups have an overall more similar vowel space than those with a lower value. Groups with higher scores are a darker shade, to provide a visual scale of similarity.

The first observation we can make is that there is a considerable amount of overlap between groups generally: the majority of pairings are rated as "same" more than $50 \%$ of the time. This is not particularly surprising, given that these participants are all native speakers of the same variety of English.

In general, we see higher rates of agreement between pairings within age categories than across: younger speakers are more like other younger speakers, and older speakers are more like other older speakers. This reinforces what we already know: that age is an important social factor with respect to vowels.

If we do not consider the column of younger trans women - who we suspect are orienting to the norms of a different speech variety (see \$2.3.2.3) - then the overall rates of sameness and difference are comparable within the two age categories, although they are clearly not identical. Among older speakers, straight men and queer women are the most different from each other, while queer women and queer men are the most alike. Among younger speakers, we also see a high rate of agreement between queer women and queer men, although straight men and queer women show greater overlap than their corresponding groups in the older category. Younger straight women and men are more similar than older straight women and men, suggesting that general gender differences are 
decreasing over time.

The cross-age comparison shows some interesting patterns. First, younger queer women have greater overlap with older queer speakers than younger queer men do; this may indicate that younger queer men are less invested in maintaining the linguistic markers of a queer identity than younger women are, and are converging on more mainstream masculinities; indeed, we see that younger queer and straight men have more in common than older queer and straight men. Second, we see a high rate of overlap between older queer women, and younger straight men and women; given how many of the vowels that we examined seem to show some kind of change over time, this pattern may suggest that among older speakers, queer women (and to a lesser extent, queer men) are early adopters of incoming forms.

Another point worth mentioning is the high overlap between older trans women and younger queer speakers, which does not seem intuitively logical. It may be that these older women - who have fairly high rates of agreement within their age cohort generally - are producing middle-of-the-pack variants for the most part, which would mean that they will overlap to a high degree with the majority of non-peripheral speaker groups. This may indicate an overall conservative vowel space for older trans women, generally steering away from extremes in favour of something fairly neutral. Recall that in some of the earlier individual vowel analyses, it was difficult to tell if these older women were producing something more masculine or feminine, according to their position relative to other groups. The IoS analysis suggests that they may in fact be producing vowels that are neither markedly masculine or feminine, or more importantly, that are neither nonmasculine or non-feminine.

The two speaker groups who have the overall lowest IoS scores are younger trans women, and older straight men. As we have seen, the younger trans women are very likely orienting to the linguistic norms of a different variety of Auckland English, so their low degrees of overlap with the other speaker groups is not unexpected.

The non-overlap of older straight men is more interesting, in that there is no particularly obvious explanation for it: they are demographically comparable to the other speakers in terms of ethnicity, education, and socioeconomic status, and the content of their interviews is likewise comparable to those of the other speakers, so it is unlikely to be an artefact of different speech styles or emotional engagement, or other pragmatic effect. It follows, then, that the differences we are seeing are a product of linguistic differentiation along social lines: older straight men may be distancing themselves from everyone else, everyone else may be distancing themselves from older straight men, or, more likely, it is some combination of the two, with different effects across different vowels. That is to say, some vowels likely signal a strong heteronormative male identity, which other groups of speakers will avoid using, while other vowels signal identities that older straight men are opting to avoid associations with (e.g., queer masculinity, or young urban femininity).

The social organisation of these avoidance strategies are discussed in greater 
Table 6.22: Ranking by similarity (shading indicative of IoS score from Table 6.21; darker indicates a higher degree of similarity)

\begin{tabular}{|c|c|c|c|c|c|c|c|c|c|c|}
\hline \multicolumn{4}{|c|}{ less similar } & & & & & & \multicolumn{2}{|c|}{ more similar } \\
\hline oqm & $\mathrm{ytw}$ & yqm & osm & $\mathrm{ytm}$ & ysw & ysm & yqw & otw & OSW & oqw \\
\hline oqw & ytw & ytm & osm & yqm & ysw & ysm & yqw & otw & OSW & oqm \\
\hline osm & ytw & ytm & ysw & ysm & yqw & oqw & yqw & osw & oqm & otw \\
\hline oSW & yqm & ysw & ytw & ytm & yqw & osm & ysm & otw & oqw & oqm \\
\hline otw & ysw & ytw & $\mathrm{ytm}$ & ysm & osm & yqm & osW & oqw & yqw & oqm \\
\hline yqm & osw & ytw & oqm & osm & oqw & ysw & otw & ytm & ysm & yqw \\
\hline yqw & osm & osw & $\mathrm{ytm}$ & $\mathrm{ytw}$ & oqw & oqm & otw & ysm & yqm & ysw \\
\hline ysm & osm & otw & oqm & osw & ytw & oqw & yqm & ytm & ysw & yqw \\
\hline ysw & ytw & osm & osw & otw & oqm & yqm & $\mathrm{ytm}$ & oqw & ysm & yqw \\
\hline ytm & osm & oqw & ytw & oqm & osw & otw & yqw & ysw & yqm & ysm \\
\hline ytw & osm & oqw & yqm & ysw & oqm & $\mathrm{ytm}$ & osw & otw & yqw & ysm \\
\hline
\end{tabular}

detail in §7.4; the IoS methodology does not offer insight into particulars, but rather shows general patterns. What is clear from the IoS table, however, is that traditional linguistic approaches of treating older (straight) men as representative of their language variety paints an inaccurate picture of the speech community as a whole.

\subsubsection{Ranking by IoS score}

While the IoS table in the previous section gives us a numerical sense of the degree of overlap, and allows a quick comparison of any two groups, it is less instinctively easy to get a sense of how any given gendered identity group compares to the rest of gendered space. A more accessible way to look at the Index of Similarity data is to rank the groups according to how similar or dissimilar they are. This is summarised in Table 6.22.

Here, it is visually easier to see that older straight men and younger trans women have the least overlap with other groups. What we also see from this presentation is that younger queer women have the highest degree of overall similarity with other groups, in that there is no group with whom they do not overlap at least $50 \%$ of the time. The groups that they have particularly high agreement with cross age, sexuality, and gender lines, although they do show similarity with other queer speakers more than $70 \%$ of the time. Their lack of strong disagreement with any group suggests that their vowel space is probably quite average, in the sense that it would be towards the middle of a normal distribution more often than not. This middle-of-the-road range of production values may contribute to some extent to the lack of a strong stereotype of how queer women sound, particularly younger queer women: they sound quite a lot like just about everyone.

One stereotype that seems at least partially supported by this data is that of queer men 'sounding like women': for older queer men at least, the four groups that they are most similar to are all women, and include all of the groups of women in their age cohort. Of course, there is more to 'sounding like women' than having a highly similar vowel 
space, and indeed the specifics of vowel production are generally not mentioned in stereotypical descriptions of 'how gay men sound', so this is clearly not a complete picture. Nevertheless, this high degree of overlap of vowel space between older queer men and older women points to the fact that there may be some linguistic basis to at least part of this pervasive stereotype. It is also interesting to note that this pattern is not evident for younger queer men: yes, they show a high similarity index with queer women, but they also show high agreement with younger straight and trans men. The productivity of vowels as a site for indexing queer masculinity seems to be decreasing over time in Auckland.

Overall, older speakers are more similar to other older speakers, and younger speakers to other younger speakers (the three right-most columns in Table 6.22 generally agree in age with the reference group, although there are exceptions), suggesting that age is an overriding factor in vowel variation.

\subsection{Discussion}

Any generalisations drawn from the Index of Similarity are necessarily broad, as this method of summarising the multiple comparisons has not been subjected to the kinds of statistical scrutiny that more entrenched approaches have. There may be differences selected as statistically significant in the analyses that fed into the IoS which are more a product of large sample sizes than a meaningful difference in the real world.

Nevertheless, the IoS is still a useful tool for providing an indicative picture of how similar or dissimilar the vowel spaces are overall across the multiple gender groupings in this project, and as it is a simple metric to use, it may prove useful in other studies of relatively large systems of variation

Across both the IoS and the individual analyses, we have seen that age is an important factor with respect to vowels in NZE. This may partly be due to different social factors operating in the two age groups, or it may be that phonetic changes exerting an effect on the younger speakers have not spread to the older ones. Given the analyses discussed in previous sections, it is likely that for some vowels it is the former, and for others it is the latter.

\subsubsection{General discussion of New Zealand English vowels}

As previous research has also shown, the vowel system of New Zealand English is clearly still undergoing changes. We have seen that some of these changes follow the pattern of sound change in general - that is, with women ahead of men generally, and younger people ahead of older people generally. We have also seen that, with some vowels, this relatively straightforward pattern is complicated by gender and sexuality effects, demonstrating that for these vowels, it is possible to communicate some aspect of 


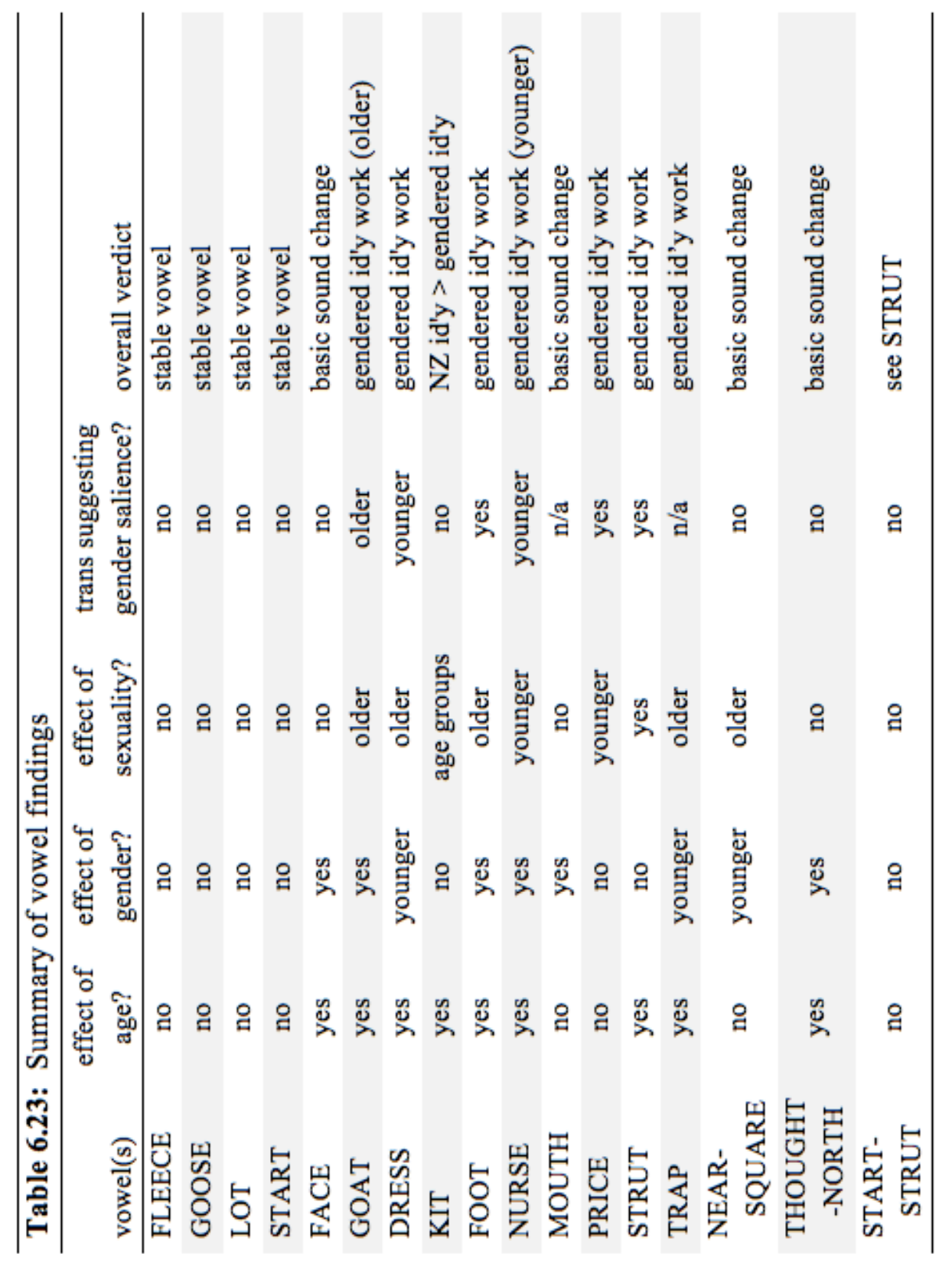


identity, whether it is an affiliation with another group, or a disaffiliation. The degree of gendered salience for any given vowel relied on an analysis of the linguistic behaviour of the transsexual participants, on the basis that trans people are very attuned to social signals of gendered identity, and that they will only make changes (whether conscious and deliberate or unconscious and unnoticed) to the vowels that are actually relevant to gender in the speech community.

A brief overview of the vowel analyses is provided in Table 6.23. For each vowel (or in some cases, pair of vowels) that has been discussed in this chapter, this table indicates whether there was an age effect apparent in the data, a gender effect, and a sexuality effect, as well as whether the behaviour of the trans speakers suggested that the vowel is a potential site for gendered identity work. Additionally, an overall 'verdict' on the vowel is also provided.

It stands to reason that not all vowels will be used for gendered identity work, since there are many other facets of identity that people may wish to convey through language choice (as noted, KIT is a highly salient marker of New Zealand identity), and there are a finite number of vowels available. Additionally, there are several nonlinguistic ways to encode gender (as there are for encoding other aspects of identity as well), so it would be inefficient to have a "one channel, one aspect of identity" approach. The Index of Similarity found overall high rates of agreement between the gender groups, which further supports the idea that not all vowels are doing gendered identity work.

In fact, we expect agreement between different avenues of presentation (e.g., dress, demeanour, and speech), and find it strange when they fail to converge. If we spread our identity performance across different channels, then it follows that we are able to communicate more than one identity per channel: my own style of speech likely indexes the fact that I am Canadian, that I identify as a man, that I am educated, and that I am a bit of a nerd. Under this interpretation, we can think of the available vowel space as one channel, with different bandwidths (i.e., different vowels) used to communicate different aspects of identity.

That gendered identity can be encoded through vowels has been shown in studies in other varieties of English (e.g., Podesva 2011; Rendall, Vasey \& McKenzie 2008; Munson \& Zimmerman 2006), so it should be unsurprising that it can also be seen in New Zealand English. However, it does not appear to be the same subset of vowels that are used for this purpose in New Zealand and North American varieties of English; articulatory and phonemic differences may make some vowels more available for gendered identity work in different varieties of English. Alternatively, it could be that gendered identity is subordinate generally to national identity, so the (metaphorical) firstpass across the vowel space initially tags some vowels as national (or regional) markers, and other identity fields are distributed across the remainder of the available vowel space.

The question, then, is how to pick out which vowels are likely sites for the identity work we are interested in - in this case, gendered identity. The answer may be as simple as examining the behaviour of those who are highly attuned to the identity we 
want to study. The inclusion of transsexual people in this study makes this simple enough, as trans people are generally finely tuned to the social norms of gender where they live. This is the result of years of both conscious and unconscious examination of what constitutes masculinity and femininity in their community, and an exploration of the ways that they can index their own gendered identities before they are able to access medical interventions to transition. Even those who identify as transgender rather than transsexual - who may opt not to medically transition because that is not a meaningful step in their own journey to full self-expression - are likely to be hyper-aware of gender norms and gender-indexing behaviours, often because, from a young age, they had an awareness of otherness in themselves, or they were overtly criticised for 'doing it wrong' at some point in their lives. For whatever particular reasons, people who do not have a tidy congruence between the sex assigned to them at birth and their own sense of gendered identity generally have a highly detailed, and generally accurate, impression of what is and is not salient to gender in their community.

To take advantage of this insider knowledge about whether a particular vowel is part of the gender 'toolkit' for Auckland, we can look at who the trans participants are most like. If they are producing vowels that are more closely aligned with those of their sex assigned at birth, then we can infer that this particular vowel does not carry much in the way of gendering significance - it has not crossed the perceptual threshold of indexing gender strongly enough to trigger a change, conscious or unconscious. On the other hand, when trans participants have adopted an articulation closer to their identified gender, then it is likely that the vowel in question is indexing gendered identity in some way.

As Table 6.23 above demonstrates, this approach seems valid, even with the restricted set of trans participants available in this study. With no older trans men, and with younger trans women who are possibly orienting to a different linguistic standard, it is hard to say definitively that the patterns we are seeing are robust and reliable.

Nevertheless, although further study with a wider representation of trans people is needed to confirm these observations, it seems likely that trans people are good indicators of the gender indexing properties of phonetic variables. This is discussed more generally in chapter seven.

In fact, the broad idea underlying this approach can, in principle, be extended to other facets of identity, as well. Trans people are particularly well-positioned with respect to gender, but presumably for other identities, similarly situated people can be found. As an example, children of immigrants may be good indicators of the sociolinguistic markers of their heritage identities, as they are likely to be engaged in a complex negotiation of identity as both citizens of their adopted country and of their cultural homeland. In these circumstances, they may be able to filter out the linguistic cues that are important in expressing cultural identity from those that simply express second language proficiency. 
The social conditions that might produce such index-indicating individuals would be:

(a) a strong social force driving them to be read as authentically $\mathrm{X}$

(b) an exposure to the norms of the $\mathrm{X}$ community that is long enough to ensure that their reading of those norms is accurate

(cf. Le Page \& Tabouret-Keller 1985)

In the case of transsexual persons, (a) is fulfilled by the desire to have their gender accurately identified, and (b) by the fact that they have been immersed in their culture and community for their entire lives. In the hypothetical case of the children of immigrants, (a) would be fulfilled by their desire to be able to fit into, and move freely between, the two cultures that dominate their lives, while (b) would be satisfied by growing up with influence from both their parents' culture and the culture of the country they live in.

\subsubsection{Some patterns}

There are a handful of patterns that show up more than once, and are worth making note of. Among younger queer speakers, for example, we see a trend towards neutralisation of gender differences, while for older queer speakers, we see traditional stereotypes (gay men sound like women, lesbians sound like men) reflected in the production data. Although there are exceptions to this trend, it nevertheless seems to be that the queer community of older participants is a more gendered space (at least linguistically) than that of younger participants. This linguistic change is likely due to general changes in the social climate of New Zealand, particularly with the social upheavals of the 1980s. This is discussed in greater detail in chapter two.

Another pattern to note is the occasional parallel between younger straight men and younger trans women. As discussed in depth in \$2.3.2.3 and mentioned throughout this chapter, the younger trans women in this study are speakers of Pasifika English, and come from South Auckland, a suburb that is strongly associated with both Maori and Pasifika communities and, in the popular imagination, with a tough gang culture (Duhamel \& Meyerhoff 2015). The occasional linguistic similarities between younger straight men and younger trans women in the Auckland Trans Corpus (e.g., with NURSE, TRAP, and STRUT) may be evidence that these men are affiliating with the toughness and masculinity of South Auckland through their linguistic practice. This is not to say that they are emulating trans women in particular, but rather that these trans women may be representative of the norms of South Auckland English, and South Auckland English is associated with qualities that appeal to the younger straight men (see §7.4.4). Unfortunately, without a corpus of Pasifika English in Auckland (or of South Auckland more generally), we cannot know how representative of this variety the speech of these young trans women is, so this possible indexical convergence is speculative at this point. 


\subsubsection{Sources of differences between this and previous studies}

In several instances, the data in this study is a marked departure from the previously reported literature on New Zealand English vowels. There are a number of possible reasons for these difference, possibly the most important being that this data was collected through spontaneous speech, as opposed to the more commonly used read speech or citation forms elicited in much of the previous work. Additionally, there are potential differences due to the time and place of the recordings: the data for the Auckland Trans Corpus was gathered in and around Auckland, between 2014 and 2016, while many of the previous studies used data from the Christchurch area, collected in the mid- to late-1990s. As the vowel system of New Zealand English is known to be undergoing changes, these differences in time depth and geography may be impacting the measured vowel system, in spite of the general sense of little regional variation in New Zealand English overall. Finally, it should also be noted that the interviewer for the entirety of the Auckland Trans Corpus was not a native speaker of New Zealand English, so there may have been some kind of accommodation going on the part of the participants, which may also account for some of the observed differences from previous studies. However, because the interviewer was the same person for all of the interviews, hopefully such effects are consistent enough across speakers that the data is at least (corpus-) internally comparable.

In the next chapter, we will take a step back from the individual variables that we have been examining, and consider some of the broader patterns that arise out of the study as a whole, as well as some of the methodological implications of adopting a liminality framework. 


\section{Chapter seven: Synthesis}

\subsection{Introduction}

This thesis has been ambitious in both its objectives and in its methodological approach. It examines variation within three different linguistic systems, with a view to investigating the social encoding of gendered identity. In the course of this, it has also introduced a new approach for assessing the interpretable social meaning of linguistic variation: liminality. This chapter will discuss some of the broad patterns that we observed across the three analysis chapters, and draw some generalisations about approaches to the study of linguistic variation and the ways that social changes are mirrored in linguistic practice. It will also consider the outcomes of this exploratory foray into liminality, and the implications that this approach may have for future variationist study.

\subsection{Variation within a system}

Rather than isolate one or two variables as potentially interesting with respect to gendered identity, this study is structured so that it examines variation in systems of variables. This has three main advantages. First, by casting the net widely, it increases the chances of identifying all, not just some, of the variables that we are interested in. Second, it allows us to contextualise the variation that we observe against the backdrop of the system as whole; and third, it arguably presents a more realistic perspective on how we process language - we do not atomistically decompose the input signal, but rather we process it as a unit that spans different levels simultaneously (e.g., lexical, segmental, syntactic, prosodic, etc.). As automatic and semi-automatic software for data extraction and coding become more sensitive and productive, this approach will likely become more common, although it will necessitate developing new analytical approaches designed to handle the large volumes of data that these linguistic tools can generate.

\subsubsection{Dimensionality and boundedness}

This project looked at three distinct linguistic systems - adjectival modification, sibilants, and vowels - each one operating within a different possible variation space. The adjectival modifiers (intensifiers and moderators) are a lexical system of variation, and are essentially unbounded: there is no particular limit on what could eventually become an adjectival modifier, given enough time and innovation on the part of speakers. The range of variation that we see in unbounded systems is functionally infinite: there may be practical limitations on what may become grammaticalised as an intensifier (a 
seventeen-syllable word, for example, seems unlikely), but there is enormous scope for innovation and creativity in the system itself. While an incoming form may displace a previous one as the preferred variant in the speech community, competing forms can coexist (e.g., Ito \& Tagliamonte 2003) without forcing a reanalysis or a restructuring of the system of modifiers. A new form is unlikely to change either the fundamental properties of existing forms (i.e., while very has undergone semantic bleaching as part of its grammaticalisation, we would not say that its meaning has changed because of any other intensifiers), or indeed the nature of adjectival modification as a syntactic unit. Thus stupendously good can be used alongside really good and unbelievably stupidly good, even by the same speaker. In this project, although there were majority variants, there was nevertheless a considerable amount of individual and idiosyncratic variation in the data, more so for the intensifiers than for the moderators.

In contrast, vowels represent a closed system, bounded by the articulatory requirements of producing them: there are physiological limits to the configurations of tongue, lips, and jaw. With vowels being characterised chiefly by their F1 (tongue height) and F2 (tongue frontness-backness), and with the vowel space of NZE being particularly densely populated, substantial changes in one vowel can trigger changes in others. That is to say, if one vowel fronts into the acoustic space occupied by another, that second vowel may be fronted even more to maintain an F2 distinction between the two phonemes, or particularly if the acoustic space that this second vowel would be fronting into is already populated - it may change height, so that there is still a distinction maintained between the phonemes, albeit along a different acoustic dimension. The structural constraints on a two-dimensionally-bounded system can result in chain-shifts such as the Great Vowel Shift affecting English in the $14^{\text {th }}$ through $17^{\text {th }}$ centuries, as well as the more recent Northern Cities Shift in the USA (e.g., Labov, Yaeger \& Steiner 1972; Eckert 1989), the Short Front Vowel Shift in NZE (e.g., Batterham 1995; Watson, Maclagan \& Harrington 2000), and the Canadian Vowel Shift (e.g., Clarke, Elms \& Youssef 1995). These cyclic shifts were initiated through small articulatory changes that triggered a series of further changes to the vowel system along both F1 and F2 dimensions, as well as to vowel duration and a few instances of monophthongisation and diphthongisation.

In the data presented in this project, we saw evidence in support of ongoing change to the vowel system of NZE, sometimes in line with observations and predictions from previous researchers (e.g., FOOT fronting, KIT retraction and lowering), but also sometimes in unexpected directions (e.g., the apparent lowering of DRESS among younger speakers, the unmerging of NEAR and SQUARE). These observations may point to one possible property of a bounded system: boundedness imposes limits on the range of short-term variation. For example, DRESS can only raise so far before it becomes phonetically indistinguishable from FLEECE, so either (1) it must stop raising, (2) it must merge with FLEECE, or (3) it must push FLEECE in a new direction. The second and the third of these options are longer-term projects that would likely be 
unobservable in a study with short time-depth. Given the ubiquity of variation in natural language data, the first option also seems unlikely, since it predicts that we would see less variation than we do in this case.

Instead, it may be that variables have a certain envelope of (in this case) phonetic space in which they can oscillate, without necessary structural consequences for the systems in which they operate. Thus DRESS, having hit the maximum height limit imposed by FLEECE, cannot raise any further, leaving speakers no option but to lower their realisation if they want to make indexical use of variation with this vowel. Over several generations, the centre of these oscillations may move along the more linear trajectory observed in systematic chain-shifts, but in the immediate short-term, we observe the variation that occurs on a small scale, and which may at any given moment seem counter-intuitive. Recall that we also noted TRAP extending unexpectedly downwards in this data, although it is different groups of speakers producing these lowered realisations than are observed lowering DRESS. Given that it is well-established in NZE that the short front vowels (TRAP, DRESS, KIT) move in inter-related ways, when we see these two vowels apparently breaking with well-documented trends, it is worth questioning why we see this happening. I am not suggesting that this pattern is evidence of an overall reversal of the raising of the short front vowels in NZE, but rather that this synchronic variation is part of a set of smaller-scale perturbations (perhaps unobserved in previous studies because of differences in participant demographics or in time scales) that is ever-present, and is independent of larger structural changes to the system. This idea of oscillation within a delimited space may also go some way to explaining the contradictory findings in previous literature with respect to NEAR and SQUARE in NZE (see §6.3.5.1). Regardless of the time-depth of previous studies (in terms of either chronological time, or the window of time spanned by the study), variation has always been reported with respect to these two vowels, although there has been disagreement about which direction they seem to be merging in: towards NEAR, or towards SQUARE. Rather than trying to reconcile these various findings, perhaps we should take these conflicting patterns as evidence of synchronic oscillation within the prescribed space occupied by NEAR and SQUARE.

Sibilants, as a subsystem of fricatives more generally, are also a bounded system, but they are much more constrained in the freedom of movement for any given segment. Since they are principally differentiated along only one dimension (place of articulation), the articulatory limits are even more pronounced. As the articulation of any given segment is either fronted or retracted, it too may begin to encroach on another segment; the difference between fricatives and vowels is that, while vowels can shift in two dimensions to maintain acoustic separation, fricatives run out of space: the lips are the absolute limit of how fronted a fricative can be (e.g., $[\phi, \beta])$, and maximally retracted fricatives are at the glottis (e.g., [h, h]). We do not see cyclic, chain-style shifts in fricatives because at some point, segments would be lost - either merged with an adjacent 
segment, or dropped altogether as they get pushed out the front or the back of the articulatory space: either would result in a reduction in the number of phonemes. Note that lateral fricatives in theory could represent a second dimension to fricative space, but they do not seem to operate that way, so it seems fair to characterise the fricatives as onedimensional with regards to articulation.

The one-dimensional space of the sibilants seems to be associated with generally more stable systems of variation. That is to say, we see differences in articulation - and in the social stereotyped associations with those places of articulation (e.g., the idea of gay men lisping, which when performed as a parody often involves fronting the $/ \mathrm{s} /$ to a dental articulation like $[\theta]$ ) - but these don't seem likely to produce systemic shifts, and indeed we see no evidence in this project of $/ \mathrm{s} /$-fronting being associated with a concomitant fronting of $/ \mathrm{J} /$, which we might predict if the fronting was associated with a system-wide shift. An examination of the sibilants within the larger fricative space would be needed to provide a stronger argument; however, as the next fricatives forward of /s/ involve the teeth, it is hard to see that there is much articulatory opportunity for incremental movement outside of the sibilants.

These different limits of dimensionality and boundedness will constrain the types of variation and change that we observe across these systems. While this project has only looked at three systems (each with a different set of structural constraints), developing a typology of variation in systems may prove to be a fruitful area for future research, with potentially far-reaching theoretical and methodological implications in how we understand linguistic variation.

\subsubsection{Contextualising variation}

Understanding the variation that we observe in any of these systems relies on understanding the system as a whole, and contextualising any given variable within that system. Within the vowel space, for example, we saw that some vowels are relatively stable across age and gender groups, while others show clear trends that point to a trajectory of change. FOOT is in the process of fronting, for example, and NURSE is involved in something complex, which may be incorporating movement, rhoticity, and rounding - although it is not precisely the same groups of speakers involved in these two processes, suggesting that it might not be a straightforward chain-shift, but rather an oscillation modulated by other (potentially social) factors. Alternatively, since this project uses a more detailed breakdown of gender than previous studies have, this kind of pattern may be typical of sound change: there may be fine-grained degrees of synchronic variation in the speech community undergoing change, where social factors intervene differently with different variables, and these factors may simply disappear under the aggregate nature of most analyses.

In a similar vein, the gendered indexicality of /s/ only becomes apparent because

of a corresponding absence of gendered effect in $/ \mathrm{J} /$ : it is not simply that younger women 
produce fronted fricatives relative to other groups of speakers, but that $/ \mathrm{s} /$ in particular does gendered work that $/ \delta /$ does not. This observation allowed us to propose that it is the secondary turbulence, created by proximity to the upper teeth, that creates the interpretable gendered cues. This was reinforced by the finding that although $/ \mathrm{z} / \mathrm{shows}$ a similar distribution across the age groups, its Centre of Gravity (CofG) measurements seem retracted for its putative place of articulation, which seems to correlate with the fact that /z/ does not carry the same degree of interpretable social meaning, as /s/ - that is to say, it does not seem to be part of the consciously accessible linguistic repertoire, but nevertheless seems to be available for gendered indexicality.

These more detailed discussions of the variation that we observe are only possible because we have a sense of the linguistic backdrop against which these particular variables operate. Although some studies (e.g., Schwartz 1968; Ingemann 1968) have noted that we can deduce certain speaker characteristics from extremely limited exposure to segmental stimuli, the reality is that we generally encounter more than isolated segments of speech. When we try to identify a speaker's accent, for example, we may be disproportionately weighting certain features of their speech in our assessment, but we are certainly hearing more than just those features. Outside of contrived laboratory experiments, it is extremely unlikely that we would only hear two vowels, for example, in natural speech: in deciding how (relatively) centralised or lowered KIT is, for example, we are situating it relative to the other vowels in a speaker's vowel space. We are making use of their entire system of vowels, even if we are only attuning to one in particular.

The analyses presented in this thesis has considered each variable system independently. Ideally, the next step would be to develop methods for studying covariation across disparate systems; and indeed, recent work has begun to move in this direction (e.g., the Hinskens \& Guy 2016 special issue of Lingua; Meyerhoff \& Klaere 2017). The problem of comparison across systems with very different structural properties is a challenging and interesting area for the variationist sociolinguistic enterprise, and it is the logical next development in the field. This thesis has provided the necessary descriptive groundwork for future developments in this direction, and proposed a broad typology for comparing systems.

\subsection{Three waves of variation revisited}

This study is positioned within what Eckert (2012) would class as second-wave variationist research: the focus is on the speech community, and the local constructions of identity within that community. It also takes the position that social category boundaries are reflected in linguistic practice, because the unit of analysis - groups of speakers who share certain characteristics - is too large to explore variation as a constitutive, stylistic enterprise undertaken by the individuals who comprise those groups. 


\subsubsection{Operationalising gendered identity in this project}

This approach necessitates that speakers be categorised, which introduces a set of assumptions about homogeneity within those categories that may or may not be warranted. In chapter one, I discussed the criteria used to categorise participants into groups according to age (older/younger), identity (straight/queer/trans), and gender (men/women), and I argued that because I was using engagement with community-level organisations to define queerness, this strategic essentialism was not unwarranted. People were categorised as queer if they were engaged in the social exercise of being queer: being involved in queer organisations and social networks, and counting their queerness as an important aspect of their identity. Participants were considered transsexual if they were engaged in a consistent, coherent project of bringing their identities into alignment with socially-reified gender categories that are different from those they were socialised into. These categories seem justified by the social fabric of the speech community, but the question was whether they actually analytically justified. That is, to what extent is there evidence that these categories have any linguistic basis?

In the multivariate analyses presented throughout the previous chapters, multiple breakdowns of gendered identity were tested. Statistical models were built using the composite (age $\mathrm{x}$ identity $\mathrm{x}$ gender) labels (e.g., younger straight women), as well as ones using each of these factors separately (age, identity, and gender). These models also tested two-way interactions (i.e., age $\mathrm{x}$ identity, age $\mathrm{x}$ gender, and identity $\mathrm{x}$ gender). In the overwhelming majority of the cases, the composite identity provided the best model of the observed data (compared via log-likelihood tests built into the statistical package, designed specifically to test models against each other). Speaker was also included as a random effect in each of these models, which meant that inter-speaker variation within each gendered identity category was accounted for in the model. The fact that the composite categorisation was nevertheless retained in the statistical models strongly suggests that there is some independent merit to the way that participants were grouped.

Of course, this does not mean that we as researchers can a priori catalogue any group of speakers according to analytically convenient categories. There were strong ethnographic and demographic reasons to expect that the speakers grouped together here would share certain characteristics, both social and linguistic. But this does show that social categories are relevant to community-level analysis, and that the assumptions that we make about homogeneity for the purposes of a second-wave analysis are not necessarily unfounded. This study has also shown that second-wave variationist study can continue to make meaningful contributions to the study of linguistic variation, particularly in the service of exploring new descriptive directions such as the framework of liminality that this thesis has proposed. 


\subsubsection{Second-wave platform for supporting third-wave studies}

As discussed in $\S 1.2 .3$, the three-waves typology of variationist sociolinguistic research makes a lot of sense if we take the three waves as a chronological succession of studies in the same speech community, or at least in speech communities that are closelyenough related that we can be confident in generalising between them. First-wave studies consider variation very generally: what is going on in the language system as a whole? Second wave studies dig a bit deeper: how does this variation relate to particular social groups in the speech community? And third wave studies zoom in to the level of the individual: how do speakers make use of that variation to construct and interpret identity? Without the second-wave stage of research, the third-wave questions become harder to answer. Unless we know that group $\mathrm{X}$ does linguistic thing $\mathrm{Y}$, and that this information is accessible (whether consciously or unconsciously) to the individual, then it is difficult to construct a coherent argument about the stylistic use of $\mathrm{Y}$ to do an X-like identity.

As a variety of English, New Zealand English (NZE) has been extensively studied, from a range of research perspectives. Historical changes have been a major focus of NZE studies (e.g., Gordon et al. 2004), particularly with respect to phonetics and phonology (e.g., Bauer \& Warren 2004a), as have the characteristics that make NZE a variety distinct from its neighbours. On the mid-range scale, ethnicity (e.g., Holmes \& Ainsworth 1996; Bell 2000) and region (e.g., Marsden 2013; Bartlett 2003) have featured prominently as social dimensions of analysis. And although gender has certainly been considered at this scale of analysis, it has largely focused on the role of gender in diachronic sound change, rather than on gender as a driver of synchronic variation. This narrower focus on gender has been more pronounced in smaller-scale interactional and discourse analysis studies (e.g., Holmes \& Marra 2004; Bayard \& Krishnayya 2001), but a systematic variationist approach has not really been applied to date.

This project, with its feet firmly planted in the second-wave tradition, can provide a resource to support future third-wave variationist work on gender in Auckland, and potentially in New Zealand more broadly. Given the community-level associations highlighted within these particular systems of variation, there is ample opportunity to focus on variation at the level of the individual - across different social contexts and speech styles - to construct a much more detailed account of the multiple indexicalities bound up these variables.

\subsection{Social realities reflected in linguistic practice}

One of the goals at the outset of this project was to investigate the ways that we draw on linguistic resources to present our gendered identity. The Auckland Trans Corpus (ATC) was designed with this particular research aim in mind, and includes a range of gendered identities with enough group-internal consistency that they can be meaningfully treated as 
coherent social identities, in spite of the differences that we will inevitably find between individuals. We did this by drawing on pre-existing social networks as much as possible, and allowing people's self-articulated identities - particularly for queer and trans participants - to play a major role in how they were classified.

Across the different systems of variation that we worked with, we observed a number of different patterns, as we would expect with such structurally different sets of variables. As we discussed in $\S 7.2 .1$ above, the types of variation that we are likely to observe seem to be at least partly constrained by the nature of the system itself, and there is no particular reason to think that systems with different restrictions will be entirely comparable. Nevertheless, there were some associations and patterns that recurred often enough to be worth commenting on here, points of linguistic divergences and convergences that may reflect more generalisable indexical relationships between social identities and linguistic practice.

\subsubsection{Divergence: older straight men and younger women}

Perhaps the most striking overall finding in this project is the linguistic distance between older straight men and the rest of the speech community, and with younger women in particular. This linguistic distance reflects an ideological distance, with older straight men positioned as the unmarked default in the heteronormative social world that they inhabit, and all other identities positioned as a departure from this norm (see §2.2.1 for a brief overview of masculinities in NZ). This observation is not new by any means. Androcentrism (Gilman 1911) has been noted across practically every sphere of human culture, often with serious consequences (see, e.g., Risberg, Johansson \& Hamberg 2009; Verdonk, Benschop, de Haes \& Largo-Janssen 2009, for gender bias in medical research). Even in sociolinguistics, one of the early gold standards was to collect data from nonmobile older rural males, because their linguistic practice was thought to be the more authentic. This may be an appropriate approach from the perspective of dialectology, if the focus of research is on the dialect (rather than on the people who speak the dialect), but also defaults to men as the unmarked, and women (and other categories of men, such as younger or mobile) as somehow producing a lesser (or at least less 'pure') version of the variety. Indeed, much of the feminist enterprise has been to destabilise this kind of androcentrism (e.g., Fraser 2009), so for the purposes of this discussion, we will treat this male-centric world-view as uncontroversial: western culture is by and large maledominated and patriarchal.

There is an interesting corollary to this perspective. If straight men are the unmarked default against which other identities are compared, then these differences must be defined relative to the behaviours and practices of straight men. This implicitly positions these men as the observers and evaluators of such differences: as we noted above, it is people within a category who are best able to spot deviations from that category. This positioning, coupled with the androcentric view that the male experience 
is the natural and obvious one, gives straight men license to impose their schemas onto others. What this means is that straight men have the (perceived) authority to dictate the 'right' way(s) to be men, and (paradoxically) also the right way to be women (this presumably extends to other dimensions of social identity, such as ethnicity - e.g., straight white men dictating the 'right' way to be black and political, which seemed to be a common theme in much of the commentary around the Black Lives Matter movement in the US in 2016).

Thus straight men are entitled to criticise women who do the 'wrong' kind of femininity, who fail to live up to the standards that they have imposed. Young women are particularly egregious offenders in this regard: while the older straight men in this project position women as gentler and more fragile than men, and more in need of protection, these young women don't restrict themselves to this limited range of possibilities. When they deviate from this plan, they become legitimate targets for criticism and correction.

There is, of course, a chicken-and-egg problem here: are young women criticised for pushing the envelope, or are they pushing the envelope in response to criticism? That is, are straight men picking on their linguistic practice as a safe target (where they can frame their corrective attempts as being in the young woman's best interest, e.g. no one will take you seriously if you do $X$ ), or are young women doubling down in the face of being told to stop doing $X$, and doing it more? We know sociolinguistically that young women tend to be linguistic innovators (e.g., Labov 1972), including with variables that are under the radar and therefore not accessible for overt criticism. It seems likely, then, that they are also leaders in more marked variation (e.g. Labov 2001a: 274), so at least some of this critical dynamic probably stems from older straight men picking up on new linguistic features and imbuing them with negative associations. However, we also know that teenagers in societies such as New Zealand are licensed to have a certain rebelliousness, and can (and do) push back against parental instruction; the reality is likely to be a combination of both factors. That is to say, older straight men criticise younger women for their linguistic innovations as a stand-in for more direct forms of criticism, and young women respond by exaggerating the very thing that triggered the commentary in the first place.

\subsubsection{Divergence: older straight and queer men}

The heteronormative androcentric world-view not only licenses older straight men to comment on and criticise the speech of young women, it also positions them as arbiters of masculinity, which in turn licenses them to also comment on the linguistic practices of 'deviant' men - men who do not live up to the expectations of their idealised masculinity. Although there is less meta-discourse about the linguistic inadequacy of queer men than there is regarding young women, language use is clearly implicated in the stereotypes of queer masculinity. From the ever-present assertion that gay men talk like women, to the exaggerated lisp of camp performances, there is a clear speech style that 
indexes queer masculinities. Before the development of a publicly visible gay male identity, linguistic cues may well have been used to signal in-group membership in a way that was opaque to the out-group; since then, it has crystalised into a recognisable register, where it continues to signal in-group membership, but in a more transparent way. For queer men who make use of this register, it is a way to distance themselves ideologically from the heteronormative framework that is characterised by a relatively monotonic expression of masculinity.

In fact, there is a second corollary to this androcentric world-view, which follows from the first discussed above: straight men, in order to hold onto their own moral authority to dictate gendered behaviour in others, must police themselves with regard to their own presentation of masculinity. That is to say, they cannot expect to be taken seriously as real men if they exhibit any of the behaviours that they criticise in queer men (e.g., Cameron 1997; Kiesling 2005). This insight can account not only for the linguistic divergences that we have observed, but also for the smaller range of inter-speaker variation that we observed among older straight men, compared to the other groups. As more and more people break from the ordered world-view that older straight men seek to impose on the world, they become more conservative in their own linguistic practices, and end up relatively isolated from the norms of the rest of the speech community.

\subsubsection{Convergence: queer identities in vowel space}

Recall in $\S 6.3 .6$ that we developed a metric for comparing overall likeness of the vowel spaces of different groups, the Index of Similarity (IoS). There were a handful of group dyads that had very high IoS scores, which means that their vowel spaces were, statistically, almost identical. The dyads with the highest overall scores all involved queer categories of identity:

(A) older queer men $\sim$ older straight women [9/10]

(B) older queer men $\sim$ older queer women $[10 / 10]$

(C) younger queer men $\sim$ younger queer women [9/10]

(D) younger queer women $\sim$ younger straight women [9/10]

It is interesting to note that, despite the temptation to commutatively equate older straight women and older queer women, this pairing had a lower IoS [8/10] score than either (A) or (B). Similarly, younger queer men and younger straight women had a lower IoS score [7/10] than either (C) or (D). We can collapse these four pairings into three patterns (pooling $\mathrm{B}$ and $\mathrm{C}$, as they describe similar overlapping identities across different generations), and look at each in turn.

\subsubsection{Queer men $\sim$ queer women}

One of the characteristics of queerness, in its 'queerest' form, is a rejection of the hegemonic strictures of heteronormativity. It is not a rejection of the feminine and masculine per se - lesbians are women, after all, and gay men are men - but it is a 
departure from the social expectations of what it means to be a woman (wife, mother, care-giver) or a man (husband, father, provider). Viewed from the outside, then, traditional evaluations of lesbians would position them as failed women with aspirations to masculinity, and of gay men as regressive and submissive effetes. Viewed from the inside, the idealised queer person is liberated from these externally-imposed, restrictive roles.

Linguistically, whether queer men are 'aiming' for a performance of femininity and queer women 'aiming' for one of masculinity, or whether both are 'aiming' for a relatively ungendered middle ground (i.e., whether the convergence is deliberate or accidental) is in a sense immaterial. The end result is the same: the gendered extremes of the linguistic realm are neutralised among people who reject the gendered extremes socially and culturally, as well; this can account for the much of the observed linguistic similarity between queer men and queer women, within their age cohorts.

\subsubsection{Older queer men $\sim$ older straight women}

There is a pervasive and persistent stereotype that queer men 'talk like women'. Several researchers have tackled this question (e.g., Gaudio 1994; Smyth \& Rogers 2008), with generally mixed results, but with overall vowel spaces, at least, there seems to be some merit in the link. This is not to say that these two groups sound $90 \%$ identical: the vowels analysed have been normalised to correct for biological differences, after all. Rather, this tells us that they are distributing their vowels similarly, with respect to every other vowel in their respective vowel spaces.

Stereotypes aside, why might we see this convergence? Consider the social positioning of both straight women and queer men, with respect to straight men. Both groups are under scrutiny and criticism from hegemonic masculinity: women for being generally inferior but sometimes needing to be kept down, and queer men for their failure to be real men, proper men. This kind of shared external pressure may have made them natural allies, with these overlaps in experiences and outlook driving them closer together. Straight women can construct queer men as non-sexual intimates, men who do not carry an implicit threat of violence, while queer men can construct straight women as a peer group who will not hold them to cultural expectations of masculinity. The vocalic convergence - almost certainly below the radar for both groups of speakers - can be construed as a manifestation of their unconscious alignment in the face of hegemonic male evaluation.

\subsubsection{Younger queer women $\sim$ younger straight women}

These two groups of young women not only have a high IoS score, they also converged to a large extent in our analysis of adjectival modification (chapter four) and sibilants (chapter five). These convergences, rather than demanding an explanation for why they are so like one another, actually need an account for why they haven't diverged more than 
they have. After all, we see less parallelism in linguistic forms between queer and straight men, in the younger and particularly in the older cohort: why have these women not followed suit?

We have seen that straight men and queer men are positioned and constructed in a sense in opposition to each other: straight men are the unmarked norm, the presumptive default from which other identities diverge. Queer men diverge from straight men, and so are marked; the spur for this markedness is their sexuality. Women are also generally diverged from straight men, but their markedness hinges on their gender, rather than their sexuality. The social drivers for divergence do not seem to be strong enough between groups of women to force another markedness split driven by sexuality - given that they are already marked with respect to the unmarked norm, they do not further subdivide into an oppositional dyad. The fact that this holds slightly more true for younger speakers than for older ones suggests that this convergence is becoming more pronounced over time. This is potentially happening as the social effects of divergent sexualities become less marked over time; we observe a similar trend among younger queer and straight men as compared to their older counterparts, but the degree of convergence is nowhere near as advanced.

\subsubsection{Convergence: South Auckland and young masculinity}

Although the convergence of younger straight men and younger trans women is not universally noted across all of the variables we have looked at - it is largely restricted to three of the ten vowels analysed (NURSE, TRAP and STRUT) and some of the patterns noted with adjectival modification - it is nevertheless worth examining more closely because even this small number of convergences is higher than we would expect from chance alone, given the large number of gendered identity categories operationalised in this project.

On the face of it, trans women and straight men is not an obvious dyad for linguistic convergence. Younger straight men are invested in the social project of doing masculinity, whether consciously or otherwise, while trans women are very consciously invested in femininity. However, as has been mentioned previously, both of the younger trans women in the ATC are Pasifika from South Auckland: so they are in fact engaged in the project of doing a particular kind of femininity, oriented to different cultural and linguistic norms than their non-Pasifika peers. The phonetics of Pasifika English in Auckland have not been particularly well documented to date, so we do not know where in relation to other speakers of this variety these young women are positioned. Lacking any evidence to the contrary, then, we can presume that they are not wildly atypical representatives of their speech community overall (although where they would fall in a gendered map of this speech community is a different question).

So how is South Auckland Pasifika English evaluated in Auckland more generally? What are the associations that people have with this variety? A multi-year 


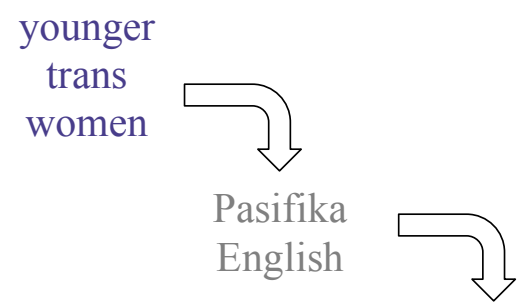

South Auckland
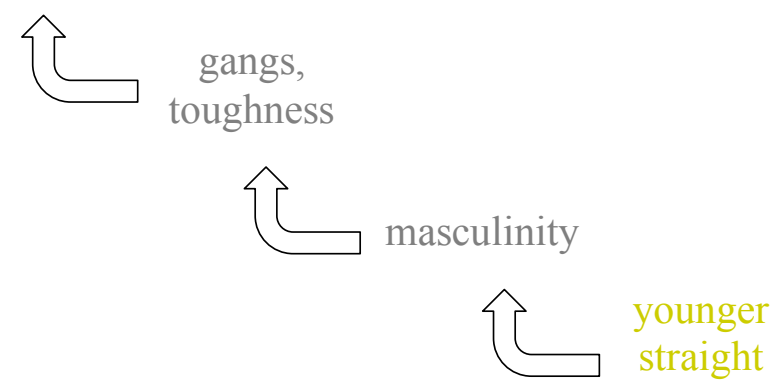

men

Figure 7.1: Chains of indexical association linking younger trans women and younger straight men

survey of language attitudes using dialect maps (Duhamel \& Meyerhoff 2015) found that the most common responses that South Auckland elicited were along ethnic lines (Pacific Islander, Maori), negative evaluations of the spoken variety (bad pronunciation, broken English, rough English, improper, slurred words), and an implicit linking with lower social class (p.243). Crucially, people also had an association with gangsters and toughness, which Eckert (2008: 465) argues (via Connell 1995; Irvine \& Gal 2000) is intrinsically and unavoidably linked with working class masculinity.

Taken together, these two chains of associations - rooted independently in masculinity and Pasifika identity, but linked through younger straight men and younger trans women in the ATC - converge on South Auckland English (Figure 7.1). We see that younger trans women are 'doing' Pasifika English, which is linked with South Auckland - this much of the chain is fairly self-evident. In the other direction, we have younger straight men 'doing' masculinity by drawing on ideologies of toughness, which are associated with South Auckland.

It is not the case that these young women are somehow 'doing' masculinity, or indeed toughness, themselves: both of them confidently, consistently, and coherently enact feminine identities. However, they are also 'doing' Pasifika identities, equally confidently and consistently. It is unfortunate that we do not have a baseline of South Auckland English to compare their speech with, to gauge the particular style of femininity that they are creating; this will be a project left for future research. In the interim, however, we will simply note this high degree of convergence between this unlikely pairing of gendered identities, and suggest that the indexical chain presented in Figure 7.1 is a strong explanatory candidate. 


\subsection{Multiple lenses of social perception}

Listeners adjust their perceptions - or their evaluation of those perceptions - based on contextual cues; these can include physical speaker characteristics such as height, weight, sex, age, and ethnicity, as well as more subtle primers, such as non-language items in the testing room (e.g., stuffed animals - see Hay \& Drager 2010). This tells us that listeners pass their perceptions through a filter of expectation before categorising them as appropriate or inappropriate. A simple non-linguistic analogy can be found in the world of fashion. Consider a woman wearing a short skirt over sheer-paneled leggings with a crop top and patterned All-Stars. The outfit itself is unexceptional - but it would seem markedly out of place if worn by a woman in her 70s, as opposed to one in her $20 \mathrm{~s}$. This is because fashion is sensitive to age: we have a cultural sense of the age-appropriateness of particular styles of clothing, and notice when someone is dressed contrary to these norms. That is to say, our assessment of how good outfit X looks is based at least in part on the characteristics of the person wearing it. A toddler in a three-piece-suit evokes a different response than a middle-aged man wearing a similar suit, because they index different things across different age groups. We can think of these sets of expectations as perceptual lenses, bringing some aspects into sharper focus than others.

In certain of the preceding variable analyses, we found it necessary to group the eleven gendered identity categories according to age or gender, before we were able to make sense of the patterns that we observed. This was independent of the analyses, which were conducted using all speakers simultaneously - but the picture was often much clearer, and it was considerably easier to understand the patterns in the data, once we had mechanically sorted the results according to the kinds of social lenses that we know we draw on as language users.

\subsubsection{Age as a perceptual lens}

The most common lens that we applied to the findings was age. With some of the vowels (e.g., for the onsets of FACE and GOAT, and to a large extent, STRUT), age emerged naturally from the data: the younger speakers had universally higher vowels (articulatorily) than the older ones, which was taken as clear evidence of a change in progress for both diphthongs. For the remaining vowels, the findings were sorted manually, and the patterns interpreted accordingly; we also considered age with respect to sibilants and adjectival modifiers, as well. This approach makes sense both in terms of the cognitive strategies for speech perception, but also from a sociolinguistic point of view, as we know that when a linguistic system is undergoing change, we typically find the innovative variants being used by younger participants more than older ones. At any given time there may be more than one system in the speech community operating semiindependently, and as language change permeates the community over time, the newer systems edge out the older ones. This approach of sorting by age is a relatively coarse 
way to tease apart these competing systems. With age operationalised in a more gradient fashion, a different approach would likely be needed, but with the binary categorisation adopted in this study, sifting the results into age groups proved a straightforward way to illuminate the data.

\subsubsection{Gender as a perceptual lens}

Another substantive perceptual lens that we apply in speech perception is gender, and the results of the analysis for $/ \mathrm{s} /$ in particular make a strong case for adopting an interpretive framework involving two separate indexical fields, one if the speaker is perceived as feminine and one if the speaker is perceived as masculine. If we assume the opposite - that there is only one set of indices that is applied regardless of the gender of the speaker - then we run into some difficulties in constructing a coherent story, as we found in our discussion of sibilants in chapter five.

At first glance, this was not obvious: we saw a clear preference for higher CofG/lower Skewness measurements among women, and a lower CofG/higher Skewness for men, which suggested that we should be able to adopt a simple linear continuum with [+feminine] at one end, and [+masculine] at the other. The problems began to arise when we considered the groups of speakers who fell towards the middle of this continuum, which in the case of $/ \mathrm{s} /$ were many of the queer identities. Younger queer men and older queer women, for example, were positioned quite close together when we considered CofG and Skewness simultaneously (e.g., Figure 5.8). Although their phonetic profiles are similar, we don't necessarily want to claim that they're indexing the same things, or indeed are oriented the same way to masculinity and femininity. The queer men unambiguously identify as men, and the queer women likewise identify as women, so it is disingenuous to try to argue that their genders can somehow be conflated. We tried tweaking the endpoints of the scale, then, so that it no longer spanned between [+masculine] and [+feminine], but rather between particular styles of masculinity and femininity: in chapter five we proposed simply A and B, but perhaps [+traditional heteronormative masculinity] and [+contemporary urban femininity] are workable labels at this point in the discussion.

It became clear that we were not looking at one continuous scale any more: the two extremes now differed on more than one dimension, which made it difficult to see

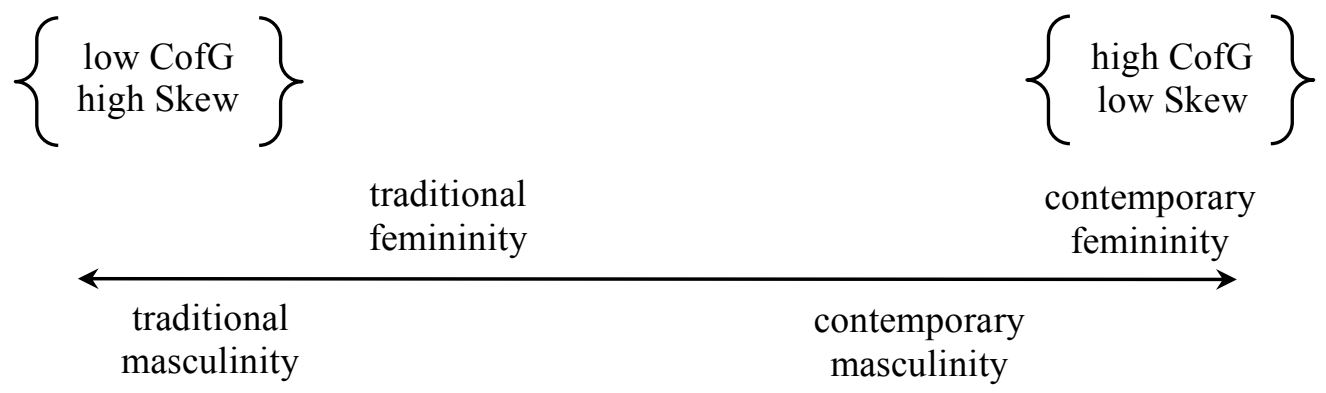

Figure 7.2: Revised schema of social interpretability for sibilants 


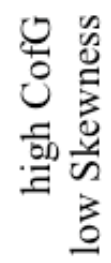

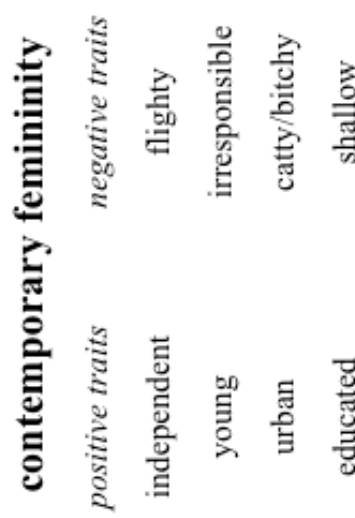

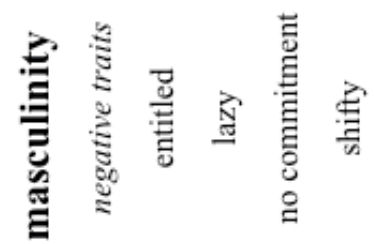

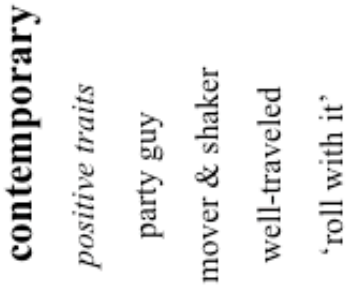

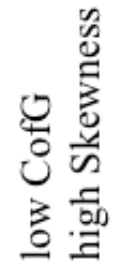

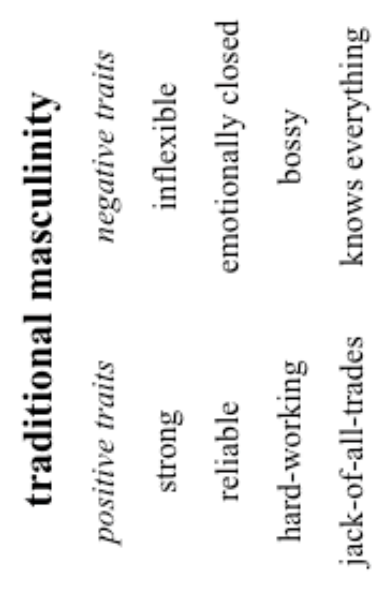

Figure 7.3: Indexical associations of gendered /s/ 
this scale as anything other than discontinuous in the middle. Instead, we conceptualised two separate scales, slightly offset with respect to each other to more closely reflect the phonetic observations. Figure 7.2 shows two opposing feminine identities, and two opposing masculine identities; separated in this way, it is much easier to see how the two extremes in each scale can be linked by an uninterrupted chain of incremental changes. Each of these scales has an accompanying indexical field, populated by the ideological baggage (positive and negative) that goes with each gendered style. A brief sketch of some of the general associations that people may have with these polar identities is presented in Figure 7.3. Note that these associations are illustrative only; we can expect considerable variation in the traits that people assign to these identities.

If someone is positively oriented to a particular identity, then they will associate with the positive traits in the indexical field; if they are negatively oriented to it, they will attune to the negative ones. Thus, following our discussion in $§ 7.4 .1$, young women will likely see themselves as [independent, urban, educated], but older straight men will more likely evaluate them as [flighty, irresponsible, shallow]. People who are neutral towards the identity will see both the positives and the negatives, and presumably those who fall somewhere between the two extremes on either scale are able to take advantage of the positive associations of both, and try to mitigate their negatives.

\subsection{Liminality and social drivers of change}

The variationist sociolinguistic enterprise has been very successful in developing theories and frameworks for studying structural language variation and change (see e.g., Labov, Ash \& Boberg 2006 for phonological change; Walker 2010 ch.6 for grammatical variation; Chambers \& Schilling 2013 for a number of studies examining variation across different systems), and the social correlates of these processes. What has received less focused attention is the question of social drivers of language change, rather than social effects of language change. Third-wave studies address the question of social meaning (see Zhang 2005; 2008 for an excellent discussion of the emergence of particular indexicalities in Beijing), but they tend to be based on variables where the social meaning of those variables is already known and is accessible to members of the speech community; these could often be classified as stereotypes in the Labovian sense. The more interesting question is: given distributional or statistical evidence of a social effect

for a given variable, how do we know whether that variable is doing social identity work, or is simply reflecting social identities as part of a trajectory of grammatical change? How can we distinguish between variation driven by social forces from that driven by other factors?

Because gender is implicated in trajectories of language change, we often find 
strongly gendered patterns with variables that are below the radar - below the level of awareness in the speech community. Of course, we find gendered patterns with variables above the level of awareness, too. So if we are interested in identifying variables where gendered effects are interpretable, and ones where they simply a byproduct of patterns of change - in other words, disentangling those doing sociolinguistic work in the speech community from those doing sociolinguistic work - our options are limited. For variables that have reached the point of stereotypes (i.e., linguistic forms that are so strongly salient for the community that they have become part of the metalinguistic and metapragmatic discourse, such as the KIT vowel in New Zealand English), this is straightforward. Are people talking about it? Then it must be doing something.

But this is quite a small inventory of variables, on balance; there is much more going on linguistically than we are consciously aware of, and some of this unconscious variation is giving us socially important information about our interlocutors. Just because we have no metalinguistic awareness of something does not mean that our brains are not processing it, and making meaning from it. How do we identify the socially meaningful non-stereotypes in a sea of complex variation?

\subsubsection{Going beyond perception studies}

As linguists, we expend a lot of energy in experimentally testing perceptions, carefully probing responses to controlled stimuli and inferring the processes underlying the observed reactions. But this is necessarily a time- and resource-heavy task, and there is a substantial gap between the stimuli used in laboratory research (designed to control for as many factors as possible) and what real people actually encounter in day-to-day interactions (noisy and chaotic systems that our brains nevertheless make sense of).

If we imagine a scenario where we have five variables that we think may be contributing to the construction of (say) queer masculinity, an experimental approach would necessitate designing and running five separate studies, each of which would require controlled target and filler stimuli, and recruiting enough participants to be confident in the interpretation of the findings. If we could find a way to get this same kind of information from production studies, such as variationist analyses of spontaneous speech, our lives would be much simpler in many ways. We could record and analyse interviews with queer men, and examine their use of our five candidate variables to see what they are doing. But here again, we hit that same wall we started with: how do we know which of these variables are interpretable as [+queer masculine] to our speakers, and which happen to pattern that way for structural or other (non)-linguistic reasons?

The question of language and gendered identity provides a good starting point for this kind of question, because of the high degree of social salience of gender in most cultures. Masculine and feminine are well-established categories, and they are popularly seen as fixed (i.e., they are an inherent and unchangeable facet of identity) and exhaustive (i.e., everybody can be unproblematically classed as either masculine or feminine). The 
fact that trans people exist - that there are people who have made a conscious decision to reinscribe their gendered identity in a way that they were not socialised to as children means that (a) the gendered system is not as straightforward as some people would like it to be, and (b) we have the opportunity to examine the linguistic practices of people who are crossing this clear category boundary. As I have proposed in this thesis, this means that we can systematically examine which variables they see as productive resources for presenting gendered identity, and which they don't.

\subsubsection{Transsexuality as a window into gendered indexicality}

Trans people, or at least the trans people included in this study ${ }^{1}$, were raised and socialised as boys or girls, and at some post-adolescent point, they transitioned to live as women and men, respectively. This is important for our purposes, because while they were children, they are likely to have acquired the locally-salient genderlect appropriate to the sex they were assigned at birth. Only after having acquired this genderlect would they have made the decision to transition, and to adopt the social roles and norms of their identified gender. This is not to imply that the decision to transition is a quick or easy one, or is conceived of one day and enacted the next; there is a generally long period of introspection and soul-searching (Brown \& Rounsley 1996; CTYS 2016) - and of careful study of the gendered norms in the community. By the time a trans person decides to transition, they have amassed and analysed a lifetime's worth observational data, and have a fairly deep understanding of the gendered differences in behaviour (including linguistic behaviour) around them. This means that they have an internal catalogue of sorts, largely unconscious (or at least hard to articulate), of the cues that the people in their speech community use to infer and evaluate the gendered identities of others.

Trans people who have been in transition for at least a year tend to have ironed out any mismatches between their observations and their ability to put those observations into practice, and have settled on a style of presentation that they are comfortable and happy with, and which aligns to a large extent with mainstream expectations of their gender. More importantly, they have triaged the linguistic variation around them, and figured out which variables they need to change as part of their transition, and which they can safely ignore. In other words, they have already done precisely what we as linguists struggle to do, either experimentally or analytically.

In this study, and particularly with respect to variation in the vowel space, we

\footnotetext{
${ }^{1}$ As trans identities become more and more mainstreamed, and people begin to transition much earlier in life, the role of early socialisation may change dramatically. In 2017, as I write this, there are parents who are supporting their pre-school-aged children in beginning to transition, which will impact on their acquisition of an appropriate genderlect. In fifteen years time, anyone wishing to reproduce this kind of study will have to specify whether their trans participants transitioned later enough in life that they will have had to change their gendered linguistic habits.
} 
saw different kinds of behaviour for trans speakers across different variables. For some vowels (e.g., FOOT, GOAT) we see evidence of trans speakers having adopted phonetic realisations in line with their identified gender, suggesting that these vowels carry interpretable social meaning: it is worth the effort to get these vowels right, because they are interpreted through a gendered lens. With other vowels (e.g., FACE, KIT) we see trans speakers retaining the phonetic realisation they would have acquired as children, suggesting that these vowels do not index gender in an interpretable way: it is not worth the effort of changing how these vowels are pronounced, because they are not interpreted as a marker of gender.

We saw a similar pattern with adjectival modifiers, particularly intensifiers. Younger trans men patterned with other young men in their use of really, but with women in their use of very, suggesting that really is the more gendered intensifier in that age group. Conversely, older trans women were feminine in their use of very and masculine in their use of really, which points to very as being the more highly gender-indexing variant among older speakers. With sibilants, we saw that younger trans participants had aligned their production with their identified gender, while older trans women were producing variants more in line with their masculine socialisation in childhood.

What is more interesting than the specific meanings of particular variables is the fact that we observe these differences at all. Our basic premise that trans people (as gender-liminal individuals) are particularly attuned to the gendered indexicality of linguistic variation, and are selectively adapting their linguistic practice to the extent that it socially worth the effort, seems to have some empirical support.

\subsubsection{Beyond transsexuality: liminal identities}

What makes transsexuals such a valuable resource in a variationist study of language and gender is the fact that they have crossed a stable and major category boundary, a property that I have called liminality, and which is closely related to Rampton's $(1995 ; 1999)$ idea of crossing. While gender and queer theorists problematise macroscopic social categories like gender, arguing that the male-female dichotomy is (a) damaging and (b) not actually representative, the reality is that for the majority of people in a culture such as New Zealand's, the categories of man and woman exhaustively divide the available gendered space. This thesis is not aspirational in the sense of trying to make the world a better or more just place: it is descriptive, interested in providing as accurate a snapshot of the real world as possible, given the analytical tools available.

In a sense, this project has been a proof-of-concept for the feasibility of using liminal identities to identify socially-indexical variables. I have focused on gender for several reasons: partly because the subject interests me; partly because the category boundaries of gender are clearly delimited in New Zealand; partly because I have been in a position to recruit transsexual participants; and partly because the liminality of my trans participants is unidirectional. That is to say, they crossed the boundary once and only 
once: this means that they are fully committed to a coherent gendered presentation, and their use of the variables studied is likely to be relatively consistent.

But gender is clearly not the only social category that one could explore with this approach. Potentially any social category boundary that it is possible to cross can also be studied, by identifying people whose identities are liminal with respect to that boundary. An obvious example is ethnicity in heritage communities. One can imagine a second- or third-generation migrant family in an immigrant country, whose children negotiate multiple ethno-cultural identities. In the home, with extended family, and in heritage community groups, such a child might be heavily invested in his maintaining his Greek cultural roots and identity; but at school, and on the rugby pitch, he might be equally invested in being a typical Kiwi boy. If we as social researchers are interested in which of the linguistic cues common to the Greek community are actually used to index a Greek identity, then our bicultural rugby player is an ideal liminal person to examine. What changes in his linguistic performance between his [+Greek] and his [+NZ] contexts? Which linguistic resources has he identified as being interpretable as [+Greek, $-\mathrm{NZ}$ ] with his peers and therefore changed to a more [+NZ, -Greek] realisation? Those variables that he has changed are the salient ones, and those that he has not adapted must not be ethnically interpretable in his speech communities.

Liminality as a framework for investigating social salience - and therefore also for identifying variables that are potentially social drivers of linguistic change - has the potential to be a productive line of research, and developing this methodological approach will be a major project for the future.

\subsubsection{Revisiting Labov's variable typology}

If the tentative conclusions of this project hold true across other dimensions of identity, and we can distinguish between two different types of social salience for linguistic variables based on the production data of liminal speakers, then we may be able to refine Labov's (1972) typology of sociolinguistic variables of indicators, markers, and stereotypes. Recall from $§ 1$ 2.2.1 that this typology is based on speaker awareness of a variable and on patterns of use: indicators are below the level of conscious awareness, and show no effect of style shifting; markers show an effect of style shifting and are therefore said to be socially interpretable (although potentially consciously inaccessible); and stereotypes are directly accessible to members of a speech community, and carry overt associations with particular groups of speakers. In this project, however, we did not consider the effect of different speaking styles, so our operationalisation of salience has been at best an approximation of Labov's typology.

This project looked at variation across different linguistic levels (lexical and phonetic, with different boundedness constraints on the possible variation space), and within these different systems, we saw evidence of different types of variables at play. This is particularly true with vowels, where the space was bounded by articulatory 
restrictions and we considered almost all of the vowels that occupied that space (the exception being CHOICE, which presented too few tokens to say anything meaningful about). That is to say, we were able to examine variation in this linguistic space more comprehensively than with either of the other variable systems, so in a sense our picture of the variation space of vowels is more developed. Vowels - with the possible exception of KIT, which acts as a shibboleth for New Zealand English - do not seem generally to reach the level of linguistic stereotype, possibly because they are so central to the communicative function of language that they are largely invisible to conscious introspection. For whatever reason, vowels seem generally positioned to operate more as indicators and markers than as stereotypes.

In this project, we noted that some vowels were relatively stable over apparent time within this study (e.g., FLEECE, LOT, START, GOOSE); we found that some vowels showed variation in the direction of change, but with no particular gendered effect (e.g., FACE); then there were variables that seemed to show a gendered effect within our liminality framework (e.g., GOAT, STRUT). If we add in the stereotype level that we have tentatively assigned to KIT, then we seem to have four distinct patterns of use. Because we have not examined any style shifting in the ATC data, we do not know to what extent the stable vowels would be counted as indicators or whether they actually are unchanging (i.e., whether or not they are technically variables in the sense of something that shows variation). For the purposes of the discussion, let us assume that they are relatively static, and therefore not accounted for in Labov's typology. The remaining three patterns map tidily onto indicators, markers, and stereotypes:

- indicators show straightforward patterns of below-the-radar change, but with no evidence of gendered indexicality

- markers show evidence of below-the-radar change and gendered indexicality

- stereotypes are overtly noticeable and accessible to the speech community In essence, then, we are potentially able to use the linguistic practice of our liminal participants to distinguish between indicators and markers. This needs to be tested against style-shifting data to see whether the different distinctions produce the same categorisation, but the principle - based on the idea of speaker awareness as a metric of variable classification - seems sound enough.

But what about the vowels that we have set aside because they seem to be stable? Recall that Labov's typology is an account of patterns of variation and change, but that here we are not looking at variables in isolation, but rather at systems of variation. If we are trying to exhaustively examine everything in that system, then we might expect to find some forms that do not seem to be varying in the moment, at least not along the dimensions that we are analysing. In Labovian terms, stable variables are ones that do not change over time, but that still show variation in the speech community (e.g., variation between velar and apical forms of -ing); that is not precisely what we mean here, so the term 'stable' is not a particularly helpful one for our purposes. It might be more fruitful 
to consider them non-variables, when we are considering the variable space as a dynamic system.

This generalisation about typology is not restricted to the vowels that we have looked at. Within the system of sibilants, we would classify /s/ as a stereotype (with, for example, the speech of queer men), /z/ as a marker (in that trans participants show preferential use of the gendered variant, but it does not seem to be consciously accessible), and $/ \mathrm{J} /$ as a non-variable. Applying this typological approach to adjectival modification is less straightforward, partly because it is not possible to account for every potential variable in an unbounded system in the same way that we can with both vowels and sibilants. As the project of liminality moves forward, and we have access to data on a greater range of variables, we will be better-positioned to refine this approach to variable classification. 


\section{Chapter eight: Reflections}

\subsection{Introduction}

This project had three main goals: (1) to investigate the performance of gendered identity through linguistic practice, (2) to explore the extent to which social changes are reflected in this linguistic practice, and (3) to introduce a new approach to assessing the accessible social meaning of variation through an examination of the linguistic practices of liminal people. This chapter will briefly revisit these aims with respect to the variables investigated, and look at some directions for future research that will follow on from the findings and methodological contributions of this project.

\subsection{Review of findings}

First and foremost, this project was conceived of as a medium for exploring gendered identity and language variation, and the degree to which the two are interconnected. It used a purpose-collected corpus of sociolinguistic interviews with straight, queer, and transsexual participants in Auckland. The participants fell into two age groups that straddled a period of substantial social upheaval in New Zealand, which provided an opportunity to investigate the effect of changing social and gender roles in linguistic practice. In chapters four, five, and six, we considered variation in three systems of variables: adjectival modification, sibilants, and the vowels of New Zealand English (NZE). The social patterns that we observed were examined under a multivariate lens, in order to account for the (structural) linguistic constraints so as to not overestimate the magnitude of the social effects that we were primarily interested in.

At the start of this project, we laid out our three main research aims as discrete points, but in fact they are fundamentally interconnected across the analyses: we drew on the liminal status (3, above) of trans speakers to highlight the gendered patterns in the data (1), which in turn showed an age effect that mirrored the kinds of social changes (2) that we predicted might have an impact on linguistic practice. In spite of this tangled relationship, it is worth revisiting our stated aims one by one, and commenting on how the patterns that we observed in the data contributed to our understanding of each of these aims.

\subsubsection{Research aim 1: Gendered identity and variation}

It is well-known in variationist sociolinguistics that social factors can exert a strong influence on the linguistic practice, both at the level of the community and at that 
of the individual. Gender is among those social factors known to play a role in language variation, and some recent work has also shown that sexuality can be encoded linguistically as well, particularly at the phonetic level. The composition of the Auckland Trans Corpus (ATC) was designed to explore the dimensions of gender (masculine/feminine), sexuality (queer/straight), and identity (cissexual/transsexual), which are grouped under the general heading of gendered identity. This corpus allows us to explore the fine-grained detail of gendered identity across three systems of variation.

In examining the system of adjectival modification, we found fewer and lessstrongly constrained gendered patterns in NZE than previous studies have found in other varieties, although this may be in due in part to a smaller data set and the effect of having so many gendered categories. We noted that there was a broad gendered reversal between the generations, particularly with the intensifier really and the moderator kind of/sort of: both are the preferred variants among older women and younger men. We also noted that the intensifier very seems to be increasingly associated with queer masculinity: it is the preferred variant by queer men in both age groups, and the factor weight gap between queer and straight men is considerably higher in the younger group than it is in the older group. So, which is a particularly strong marker of young femininity in the North American varieties of English that have been studied, does not show a comparable effect in this data.

The second system of variation that we considered was sibilants: /s/, /z/, and / $/$. We found gendered differences on $/ \mathrm{s} /$ and $/ \mathrm{z} / \mathrm{under}$ the multivariate lens, but not for $/ \mathrm{J} /$, although it also worth noting that $/ z /$ does not seem subject to the same degree of overtly accessible social meaning that /s/ does; we have tentatively posited that the social meaning is derived from the secondary source of turbulence (the front teeth), and with $/ \mathrm{z} /$ having a somewhat retracted articulation relative to $/ \mathrm{s} /$, the gendered variation may be less acoustically accessible for listeners. For both /s/ and /z/, the spectral continuum had older straight men positioned at one end, and younger women (straight, queer, and trans) at the other. These observations align with prevalent gendered stereotypes of /s/, and map onto the prevalent social tension between these two groups more generally: younger women are disproportionately subjected to criticism, particularly from older men.

Finally, we looked at vowels, where the number of analyses covered makes it difficult to summarise the general findings concisely. We noted several vowels that seemed to have a gendered effect (in particular: GOAT, DRESS, FOOT, NURSE, PRICE, STRUT and TRAP), while others were relatively stable, or could be argued to be undergoing sound change according to well-established principles and independent of gender. The Index of Similarity (IoS) metric allowed us to compare vowel spaces across different gendered groups, giving an estimate of general similarity. We found that similarity was higher within age groups than across it, and that older straight men and younger trans women (for very different reasons) had the lowest IoS scores overall; this mirrors the linguistic isolation of older straight men that we found in the study of 
sibilants. We also noted that social stereotypes about queer men 'sounding like women' were more evident among older speakers than younger ones, as were differences between men and women generally.

On the whole, this study found that quite subtle differences in linguistic practice can carry gendered information about speakers. Of course, any one of these variables in isolation would be unlikely to comprehensively signal gendered identity. However, since we don't encounter linguistic variables in isolation, but always in the context of other linguistic and social cues, each salient variable is likely to contribute to an overall impression. The perception of gender is cumulative, and more sophisticated statistical methods of modeling complex co-variation will hopefully shed light on the ways in which we signal our identity across multiple channels, and how we interpret the identity of those around us. The IoS metric is a first step towards this larger aim, but further work is still needed to bridge more typologically-distinct systems of variation.

\subsubsection{Research aim 2: Social change reflected in linguistic practice}

The late 1970s and early 1980s saw several social changes move through New Zealand society: economic reforms drove a shift towards urbanisation, which in turn had an effect on the gendered ideology. This renegotiation of masculinities and femininities was no doubt buoyed up in part by the arrival of second-wave feminism from Australia, and coincided with a series of campaigns to decriminalise homosexuality. The older participants in the ATC grew up under a more traditional gendered model, while the younger participants came of age in a markedly different environment, with increasingly mainstreamed queer identities and an ideological orientation to gender equality (although the extent to which this more egalitarian ethos has actually been implemented is a matter of some debate). Given the relationship between gender and linguistic practice, we might expect to find some of these social changes reflected in the variation that we observe across these two age groups.

The study of adjectival modification did not reveal any compelling evidence of an effect of social change. This is not to say that there were no differences in the data between the two age groups, but rather that the changes that we did see could not be clearly linked to the social changes. The reordering of the gendered patterns for really across the age groups - being preferred by older women and younger men - does not map onto any of the social changes likely to be at play between the two generations of participants in the ATC; furthermore, the usage patterns of our trans participants do not suggest a coherent set of gendered indexicalities within this system. The indexical narrowing of very with respect to queer masculinity may be a social marker of identity, but this accretion of meaning is likely largely independent of larger societal changes.

With the sibilants, we found that older straight men were acoustically removed from the other speakers, including other men. This is potentially related to a policing of masculinity in which older straight men are increasingly constrained by the loosening of 
restrictions on other expressions of masculinity: as different styles of 'doing being men' become more mainstreamed, the only means by which these hegemonic men can hold onto their moral authority is to retreat into linguistic conservatism. The second pattern that could be said to mirror social change is the difference between older straight men and both older and younger queer men. Debates in the 1980s around the decriminalisation focused largely on men, and the social (and criminal) sanctions against male homosexuality created the need for straight men to distance themselves maximally from gay men; among younger men, this distinction seems much important, with younger straight men finding humour (rather than insult or offense) in being misidentified as gay.

In our analysis of vowels, the clearest reflection of social changes was found in the IoS analysis, where there was an increased convergence generally between queer and straight participants in the younger age group, as compared with their older counterparts. This suggests that the social and political mainstreaming of queer identities in New Zealand has reduced the stigma of non-heteronormativity to the point that straight speakers are no longer making as productive use of phonetic resources to distance themselves from previously problematic identities, which further reinforces our impressions of the patterns that we observed with sibilants.

Although the social changes that we found mirrored in this data are primarily concerned with different masculinities, this is not in itself surprising. In a hegemonic structure, we would expect changes that affect those positioned closest to the centre of power (in this case, older straight men) to have broader ramifications in the system as a whole. Under the more traditional gender schema of the older participants, women were positioned as less powerful and less agentive than men generally; the further subordination of lesbians presented a smaller social difference than that between straight and gay men. The ATC is particularly well-positioned to explore the social consequences of the decriminalisation of homosexuality, and on the whole, we found that as queer identities have been mainstreamed in urban New Zealand, the reduction in social distance between queer and straight men seems to be associated with a parallel reduction in linguistic distance between these groups, as well.

\subsubsection{Research aim 3: Liminality as a new approach}

The question of assessing social meaning is a tricky one in the variationist enterprise, but it is a central one if we seriously want to engage with the question of socially-driven language variation and change. The variation that we observe more often than not correlates with at least some of the social factors relevant in the community (age, gender, class, ethnicity, etc.), even when the variation seems to be structurally (i.e., language-internally) motivated; one of the properties of language change propagating through a speech community is that some groups of speakers will tend to be innovators. This means that we cannot infer interpretable social meaning based on usage alone, 
because we cannot disambiguate between social drivers of change and social effects of structural change. The idea behind the liminality approach - of collecting data from people who have crossed a perceptually important social category boundary - is to identify the linguistic variables used as resources by liminal people to converge linguistically with their identified group(s). The variables that they manipulate are likely to be socially indexical of their identity and therefore salient in the sense that we mean it here, while those that they do not manipulate - where they are not converging as we might predict - are likely to be non-salient with respect to their liminal identity categories. In this project, we have been looking at the linguistic practices of transsexual participants, as people who have crossed the socially-reified category boundary between men and women and who are therefore finely tuned into the gendered salience of variation in their community.

In our study of adjectival modification, we found mixed evidence of trans speakers adopting the variants of their identified genders. Younger trans men were using really on par with their masculine peers, but older trans women had not adopted the preferred variant of other older women, although they were avoiding the [+queer masculine] very. Whether or not this is an avoidance strategy, on par with what Hazenberg (2012) noted in Ottawa, is difficult to tell, because their use of very is comparable to that of both straight women and straight men. It is more likely that any gendered indexicality of intensifiers in Auckland is a recent development.

The patterns that we observed in the study of sibilants were strongly suggestive of gendered indexicality for $/ \mathrm{s} /$ and $/ \mathrm{z} /$, at least among younger speakers. Younger trans men patterned closely with both queer and straight men in their age group, and younger trans women likewise patterned very closely with younger queer and straight women. The pattern that we found with older trans women was a bit harder to explain, in that their productions of $/ \mathrm{s} /$ and $/ \mathrm{z} /$ fell into a cluster with younger straight men and older queer men. It is likely that they are participating in a general distancing exercise with respect to older straight men, but they have not fully adopted a more feminine-indexing articulation. While this data clearly shows that gendered production differences for fricatives are not an innovation in New Zealand English, it does suggest that the perception of gendered meaning - its salience, in other words - may be more recent.

Our interpretation of gendered salience in the study of vowels was generally productive, although the ethnicity of the younger trans women complicated the approach somewhat, since they are almost certainly orienting to a different variety of English than the other participants (most likely either Pasifika or South Auckland). Nevertheless, on the whole, the liminality approach proved successful at identifying potentially gendersalient vowels, and also in pointing out cases where the gendered indexicality seems to be a recent innovation, or is potentially being lost.

As an analytical approach to assessing the salience of linguistic variables from production data, liminality looks promising. We have seen that trans speakers are 
selective about the variables that they use to converge with their peers along gendered lines, and which they seen unconcerned with adapting to reflect their identities. Of course, this approach does not guarantee that these variables are implicated in sociallymotivated language change, but it does flag them as potential targets for developing a model of the social drivers of language change.

\subsection{Where to next?}

While this project has found some interesting patterns with respect to gender and language, and provided a proof-of-concept for a liminality framework, it certainly does not mark the end of the research enterprise. It provides four distinct avenues to continue these investigations: triangulating these early findings; expanding the set of social dimensions of identity that can potentially benefit from a liminal approach; refining the analytical approaches we can take to consider variation in a system as opposed to in isolated variables; and continuing to investigate intersection of language and gender.

\subsubsection{Triangulation}

The findings of this project are certainly promising in terms of working towards a framework for investigating the interpretable social meaning - i.e., salience in the very broadest sense - of linguistic variables, but the fact is that we have only tackled the question from the production end, which limits the confidence with which we can generalise beyond this particular instance. So far, we have inferred the gendered meaning of particular variables based on how trans people produce them. We have reasoned that if a variable carries interpretable gender cues, then trans people will be more likely to adopt the variant that indexes their gender appropriately; if a given variable does not carry interpretable gender cues, then they will be less likely to change their production away from the variant they were socialised to use early in life. Certainly we have seen that the trans participants in the ATC do not consistently pattern (i.e., in respect of all the of the variables examined) with either their early socialised or their identified gender, but seem to have some kind of mixed system. Given that some of the observed convergences seem to line up with our expectations based on social stereotypes, it is not unreasonable to suppose that the same mechanism is also at play with less stereotyped or intuitively-obvious variables.

What is needed next is to develop perception studies that will investigate whether these variables are interpreted in speech community more broadly as indexing gender. It was not possible within the scope of this particular thesis to incorporate this kind of corroborating evidence, but this triangulation is an important component of this research programme. Ideally, the stimuli for these studies can be adapted from the production data in the ATC, so that we are testing the same speakers in the same contexts 
as we examined in the production studies. There are a number of methodological approaches available within the perceptual paradigm, but a good starting point would be some kind of indirect assessment of listener assumptions, through a study of their attitudes (e.g. Meyerhoff \& Duhamel's [2015] map task); future work in this area will benefit from collaboration with psycholinguists, and others who have a strong background in perception studies.

\subsubsection{Liminality}

Alongside the project of triangulation, we can also expand the range of social identities under examination, while also broadening the types of variables that we consider. This project looked at gendered identity in a fairly narrowly-defined social landscape: predominantly white (i.e., of European descent), well-educated urban New Zealanders in Auckland. It examined variation in two phonetic systems of variation and one lexical one, partly because high frequency of these variables makes them well-suited to quantitative analysis, but also because there was a high probability - given what has been found in studies of other varieties of English - of finding some kind of gendered pattern within these variable systems. If we widen the parameters of social identity that we take into consideration, we can also start to consider morphosyntactic and semantic fields of variation, depending on the linguistic attributes of the lects under study. The first step, then, is to identify axes of identity that are likely to produce the conditions of liminality: individual speakers who move confidently and consistently between different identities.

The first and probably most obvious place to look for such people is along lines of ethnicity, particularly in a society characterised by multi-generational immigration. Second- and third-generation children of migrant families are likely to balance competing identities: with their extended families and in traditional settings, they may be heavily invested in reproducing and maintain the social and linguistic norms of their heritage culture; when moving through the more dominant culture, they may be equally invested in cultivating a mainstream identity. For example, consider a teenager in Auckland who was born in New Zealand, but whose parents immigrated from India as children. If this teenager has been raised in a family that is culturally (and possibly linguistically) Indian, but educated in an English-language school, and socialised with their peers in a New Zealand milieu, then we can expect that they will have two distinct identities (i.e., [+Indian] and [+Kiwi]) that they can switch between at will. There may be expectations of being a 'good' Indian child in the home and in any social or cultural organisations they are part of; outside of these contexts, however, they may find it useful and easier to have a generic Kiwi identity, for example in school or at work, where the social capital of being the same as their peers could reasonably be expected to outweigh that of having a unique cultural identity.

This scenario, of course, is inherently different than the one we have been 
considering with the transsexuals in this project: the trans people in the ATC crossed the category boundary once, while our hypothetical multi-cultural teenager goes back and forth between different contexts. So what would data collection and analysis look like with our teenager, and how would their contribution to the project advance the question of social salience? Ideally, we would want recordings of this teenager's interactions across multiple social contexts: at home, with their peers, with their grandparents, at school, etc. This would give us access to multiple performances of their [+Indian] and their [+Kiwi] identities, and we could look for similarities and differences between them. We could then compare their production of [+Kiwi] with a sample of mainstream Auckland English, and look for further points of similarity and difference. Those variables where our teenager converges with Auckland English would represent the variables that are [+NZE] salient: those are the variables that our teenager has internalised as carrying ethnic/cultural information, and therefore makes changes to their own production. Those variables that do not converge on Auckland English, but that maintain their [+Indian] qualities (which will require a comparison with other speakers of Auckland Indian English), are those that do not carry meaningful, interpretable [+NZE] social cues. We can certainly expect a degree of idiosyncrasy on the part of our teenager, but if we can recruit several bi-cultural participants like our Indian-NZ teenager, we can generalise across individual speaker differences to deduce some of the [+NZE] variables that are salient at the community level. If we recruit several participants with the same bi-cultural background, we can also explore the linguistic salience of their shared heritage identity, and start to meaningfully ask, what are the minimally necessary components of doing a [+Indian] identity in New Zealand?

Another possibility for exploring liminality is around regional identity, although this is perhaps better exemplified looking at a variety of English that has more pronounced regional differences than New Zealand does. The United Kingdom would be a good place to explore this type of regional affiliation, although the normative pressure to converge on a particular variety may not be as strong as it is for migrants or ethnic minorities. Here, in fact, we might make productive use of skilled actors: which linguistic resources do they draw on to present themselves as native speakers of a different regional variety? Clearly, the use of actors would restrict the domain of our investigation to the phonetic - dialogues or monologues having been scripted by someone else entirely - but this does not limit us to segmental analysis, as suprasegmental variables (e.g., stress, rhythm, prosody, etc.) can also carry markers of regional varieties of English. In a sense, good actors are by definition liminal: they convince their audience of the authenticity of their performed identity.

The liminality approach is an extension and formalisation of an analysis of style shifting, but is applied in more categorical circumstances than style is generally construed as. Labov's (e.g., 1972) approach to style shifting was inherently gradient, while the idea of identity categories as implemented here is more discrete. For the liminality enterprise 
to work, the social categories under investigation ought to be as clearly delimited as possible; while some people argue that gender is not as categorical as others make it out to be, the social reality at the level of the speech community is that gender is treated as categorical. In the current political and social climate of increasing xenophobia, ethnicity and heritage culture are likely to become perceived as more and more categorical as well. Nevertheless, the common ground between studies of style shifting and boundarycrossing is doubtless characterised by substantial theoretical and methodological overlap, and the opportunity for cross-pollination between the two perspectives is a rich one.

The principle underlying this framework of liminality - examining the linguistic practices of people who move between socially relevant categories - is of course not restricted to varieties of English, and ultimately we would want to see that this approach holds true in other languages as well. The different cultures that are represented by different languages may also offer new types of category that would be appropriate for a liminal analysis: the limits of liminality are, in that sense, cultural rather than linguistic.

\subsubsection{Variation in systems}

The decision to examine variation in systems rather than to look at individual variables in isolation is certainly not a new one, and the approaches that researchers adopt to investigate these kinds of scaled-up variation spaces differ depending on the types of question that they are trying to answer. The Atlas of North American English (Labov, Ash \& Boberg 2006) has a phonetics-driven focus on dialectology, and considers variation in vowel spaces across the English-speaking parts of the continent. It uses multiple variables to construct isoglosses of the different regional varieties, and develops an analytical procedure for abstracting across multiple measures that "maximizes consistency or homogeneity" within each isogloss (p.42). Meyerhoff \& Klaere (2017) present an approach for clustering multiple variables to explore constructions of local (village) linguistic styles in Bequia in St Vincent and the Grenadines, arguing that the statistical models that most closely align with speaker intuitions are those that encompass multiple loci of variation simultaneously. A special issue of Lingua (Hinskens \& Guy 2016) offers a collection of analytical approaches posing similar questions: exploring variation across multiple systems, and "to what extent multiple co-existing linguistic variables have similar distributions, both internally and in the speech community at large" (Guy \& Hinskens 2016: 1). Each approach is well-suited to a particular combination of research question and data type: sociolinguistic data tends to be complex, and can lend itself to a number of different analytical approaches: principle components analysis (PCA), latent cluster analysis, and constrained correspondence analysis are some of the approaches that have been used, revealing interesting patterns in the data that would not be detected with a different type of analysis.

The most sophisticated approach to multi-variable co-variation adopted in this thesis has been the development of the Index of Similarity (§6.3.6) The relative 
simplicity of the this measure makes it appropriate to the kind of question that it is trying to answer: namely, how similar are the vowel spaces of any two gendered identity groups? In this project, we noted that there seemed to be more agreement within each age group than between them, that older straight men and younger trans women had generally lower similarity scores overall than the other groups of speakers (for different reasons), and that certain pairings had much higher similarity scores than others (which aligned with our expectations based on our understanding of the gendered social context).

The question of co-variation, and how best to model it statistically, is going to become increasingly central to the study of sociolinguistic variation, and I look forward to collaborating with statisticians in the future, outside the bounds of a doctoral thesis.

\subsubsection{Language and gender}

With two corpora of sociolinguistic interviews that include a range of gendered identities (the Auckland Trans Corpus collected for this project, and the earlier and smaller Ottawa Trans Corpus that provided the data for my MA research), I am in a good position to continue to explore the linguistic performance of gendered identity. As well as investigating a wider range of linguistic practices in both varieties of English (including, but not limited to, suprasegmental phonetic variables such as high rising terminals; interactional and pragmatic variables such as hedging strategies and discourse markers; and organisational approaches to narrative and storytelling), I would also like to examine similarities and differences in linguistic constructions of gender between them.

Canada and New Zealand are an interesting pair to consider in tandem: the settlement histories of the two countries are roughly parallel, and span more or less the same time period; and they were settled by people with comparable social and economic backgrounds, coming from similar regions of the United Kingdom. But there are nevertheless linguistic and social differences; the origin myths of New Zealand seem to codify egalitarianism as a cultural template in a way that those of Canada do not (e.g., the 'tall poppy syndrome' so prevalent in New Zealand culture is nowhere near as prevalent in Canada), and it will be interesting to see whether this has had any effect on the types of masculinities and femininities that are enacted and visible in these two cultures. There are also similar but temporally offset trajectories of queer activism in New Zealand and Canada, which may also result in differences in the linguistic encoding of queer identities. The fact that there are trans participants in both the Auckland and Ottawa corpora mean that a comparison of gendered salience is also possible: which variables index socially accessible gendered information will provide a valuable first point of contrast for evaluating the effectiveness of the liminality framework. 


\subsection{Final thoughts}

In some ways, this thesis asks as many questions as it answers, but these new questions are driven by the findings of this project. In drawing on the linguistic performance of trans people to shed light on the tricky question of gendered salience, we are also asking, What other types of salience could we investigate? In adopting a second-wave variationist approach that operationalises gender in a more nuanced and ethnographicallygrounded way than is traditional, the next question is, What other social categories might also benefit from this reframing? In looking at variation across systems rather than variables in isolation, we want to know, How else can we investigate complex phenomena in interaction?

As a launching-off point for these questions - and others not yet thought of this thesis has hopefully addressed some of the underlying questions on both the social side of sociolinguistics, as well as the linguistic one. 


\section{References}

Adank, Patti, Roel Smits \& Roeland van Hout (2004). A comparison of vowel normalization procedures for language variation research. Journal of the Acoustical Society of America, 116(5): 3099-3107.

Agha, Asif (2003). The social life of cultural value. Language \& Communication, 23: 231-273.

Agha, Asif (2005). Voice, footing, enregisterment. Journal of Linguistic Anthropology, 15: $38-59$.

Altman, Dennis (1996). On global queering. Australian Humanities Review, July issue. Last accessed 27 March 2017 via http://www.australianhumanitiesreview.org/archive/Issue-July-1996/altman.html

Avery, Jack D \& Julie M Liss (1996). Acoustic characteristics of less-masculinesounding male speech. Journal of the Acoustical Society of America, 99(6): 37383748 .

Bartlett, Christopher (2003). The Southland Variety of English: Postvocalic / $r /$ and the $B A T H$ vowel. Unpublished PhD thesis. University of Otago: Dunedin, New Zealand.

Bass, Michael (2009). Street or Shtreet? Investigating (str-) palatalisation in Colchester English. Estro: Essex Student Research Online, 1(1): 10-21.

Batterham, Margaret I (1995). "There Is Another Type Here": Some Front Vowel Variables in New Zealand English. Unpublished PhD thesis. La Trobe University: Bundoora, Australia.

Bauer, Laurie (1986). Notes on New Zealand English Phonology. English World Wide, 7: 225-258.

Bauer, Laurie \& Paul Warren (2004a). New Zealand English: phonology. In Kortmann, Schneider, Burridge, Mesthrie \& Upton (eds.), A Handbook of Varieties of

English: a Multimedia Reference Tool, volume 1, pp.580-602. Berlin: Mouton de Gruyter.

Bauer, Laurie \& Paul Warren (2004b). Curing the goat's mouth. Proceedings of the 10th Australian International Conference on Speech \& Science Technology, pp. 215220. Macquarie University: Sydney, Australia. December 8-10.

Bauer, Laurie \& Winnifred Bauer (2002). Adjective boosters in the English of young New Zealanders. Journal of English Linguistics, 30(3): 244-257.

Bauer, Laurie \& Winnifred Bauer (2002a). Playground Talk: Dialects and Change in New Zealand English. Wellington, NZ: School of Linguistics and Applied Language Studies, Victoria University of Wellington. 
Bauer, Laurie, Paul Warren, Dianne Bardsley, Marianna Kennedy \& George Major (2007). Illustrations of the IPA: New Zealand English. Journal of the International Phonetic Association, 37(1): 97-102.

Bayard, Donn \& Sateesh Krishnayya (2001). Gender, expletive use, and context: male and female expletive use in structured and unstructured conversation among New Zealand university students. Women and Language, 24(1): 1-15.

Beck, Janet Mackenzie (2010). Organic variation in the vocal apparatus. In Hardcastle, Laver \& Gibbon (eds.), The Handbook of Phonetic Sciences, $2^{\text {nd }}$ edition, pp.155201. Malden, MA \& Oxford, UK: Blackwell Publishing.

Bell, Allan (2000). Maori and Pakeha English: A case study. In Bell \& Kuiper (eds.), New Zealand English, pp.221-248. Wellington, NZ: Victoria University Press.

Besnier, Niko (2003). Crossing genders, mixing languages: The linguistic construction of transgenderism in Tonga. In Holmes \& Meyerhoff (eds.), The Handbook of Language and Gender. Malden, MA: Blackwell Publishing. 279-301.

Besnier, Niko (2004). Consumption and cosmopolitanism: Practising modernity at the second-hand marketplace in Nuku'alofa, Tonga. Anthropological Quarterly, 77(1): 7-45.

Biber, Douglas \& Susan Conrad (2001). Register variation: a corpus approach. In Schiffrin, Tannen \& Hamilton (eds.), The Handbook of Discourse Analysis (pp.175-196). Malden, MA: Blackwell.

Biber, Douglas, Stig Johansson, Geoff Leech, Susan Conrad \& Edward Finegan (1999). Longman Grammar of Spoken and Written English. Harlow: Longman.

Bird, Frances (2015). Singing out? An LGBTTQ choral community: identity, performance, and meaning. Paper presented at 2015 Sociological Association of Aotearoa (New Zealand) Annual Conference. University of Waikato, Hamilton, NZ: 10 December.

Boersma, Paul \& David Weenink (2015). Praat: doing phonetics by computer [Computer programme]. Version 5.4.17. Downloaded 21 August 2015.

Bolinger, Dwight (1972). Degree Words. The Hague: Mouton.

Bowen, Caroline (2011). Lisping - When /s/ and /z/ are hard to say. Last accessed 27 March 2017 via http://www.speech-languagetherapy.com/index.php?option=com_content\&view=article\&id=86:lisp\&catid=11 :admin\&Itemid $=120$

Brown, Keith (2006). Encyclopedia of language and linguistics, 2nd edition, volume 14. Oxford: Elsevier.

Brown, LeAnn (2009). "Not just super gay men”: Trans women, vlogs, stereotypes, \& the performance of gendered dialects. Unpublished MA memoir, University of Toronto.

Brown, Mildred \& Chloe Ann Rounsley (1996). True Selves: Understanding Transsexualism. San Francisco, CA: Jossey-Bass. 
Bucholtz, Mary (1999). “Why be normal?": Language and identity practices in a community of nerd girls. Language in Society, 28: 203-223.

Bulgin, James, Nicole Elford, Lindsay Harding, Bridget Henley, Suzanne Power \& Crystal Walters (2008). So very really variable: social patterning of intensifier use by Newfoundlanders online. Linguistica Atlantica, 29: 101-115.

Burr, Vivien (2015). Social Constructionism, 3rd ed. East Sussex and New York: Routledge.

Butler, Judith (1988). Performative acts and gender constitution: an essay in phenomenology and feminist theory. Theatre Journal, 40(4): 519-531.

Butler, Judith (1990). Gender Trouble: Feminism and the Subversion of Identity. New York: Routledge.

Cameron, Deborah (1997). Performing gender identity: Young men's talk and the construction of heterosexual masculinity. In Johnson \& Meinhof (eds.), Language and Masculinity, pp. 47-64. Oxford, UK: Oxford University Press.

Cameron, Deborah \& Don Kulick (2003). Language and Sexuality. Cambridge: Cambridge University Press.

Carlyon, Jenny \& Diana Morrow (2013). Changing Times: New Zealand Since 1945. Auckland, NZ: Auckland University Press.

Catford, JC (1968). The articulatory possibilities of man. In Malmberg (ed.), Manual of Phonetics, pp.309-333. Amsterdam: North-Holland Publishing Company.

Chambers, JK \& Natalie Schilling (eds.) (2013). The Handbook of Language Variation and Change, 2nd ed. Malden, MA \& Oxford, UK: John Wiley \& Sons.

Cinque, Guglielmo (2010). The syntax of adjectives: A comparative study. Cambridge, MA: The MIT Press.

Clark, Urszula (2004). The English West Midlands: Phonology. In Schneider, Burridge, Kortmann, Mesthrie \& Upton (eds.), A Handbook of Varieties of English (Vol. 1: Phonology), pp. 134-162. Berlin: Mouton de Gruyter.

Clarke, Sandra, Ford Elms \& Amani Youssef (1995). The third dialect of English: some Canadian evidence. Language Variation and Change, 7: 209-228.

Clifford, James (1997). Routes: Travels and Translation in the Late Twentieth Century. Cambridge MA: Harvard University Press.

Connell, Robert (1995). Masculinities. Berkely, CA: University of California Press.

Cramer, Duncan \& Dennis Howitt (2004). The SAGE Dictionary of Statistics: A Practical Resource for Students in the Social Sciences. London, UK \& Thousand Oaks, CA: SAGE Publications.

Crist, Sean (1997). Duration of onset consonants in gay male stereotyped speech. University of Pennsylvania Working Papers in Linguistics, 4(3): 53-70. 
CTYS (2016). Families in Transition: A Resource Guide for Families of Transgender Youth, $2^{\text {nd }}$ edition. Last accessed 27 March 2017 via http://www.ctys.org/wpcontent/uploads/2016/03/CTYS-FIT-Families-in-Transition-Guide-2ndedition.pdf

Daniloff, Raymond, Kim Wilcox \& M. Irene Stephens (1980). An acoustic-articulatory description of children's defective /s/ productions. Journal of Communication Disorders, 13(5): 347-363.

Disner, Sandra Ferrari (1980). Evaluation of vowel normalization procedures. Journal of the Acoustical Society of America, 67(1): 253-261.

Dixon, Robert MW (1982). Where Have All the Adjectives Gone? and other essays in Semantics and Syntax. Berlin, New York, Amsterdam: Mouton Publishers.

Duberman, Martin Bauml, Martha Vicinus \& George Chauncey, Jr (eds.) (1989). Hidden From History: Reclaiming the Gay and Lesbian Past. New York, NY: New American Library.

Dubois, Sylvie \& Barbara Horvath (1999). When the music changes, you change too: Gender and language change in Cajun English. Language Variation and Change, 11:287-313.

Duhamel, Marie-France \& Miriam Meyerhoff (2015). An end to egalitarianism? Social evaluations of language difference in New Zealand. Linguistics Vanguard, 1(1): 235-248.

Durian, David (2007). Getting [J]tronger every day? Urbanization and the sociogeographic diffusion of (str) in Columbus, $\mathrm{OH}$. University of Pennsylvania Working Papers in Linguistics, 13(2): 65-79.

Eckert, Penelope (1989). Jocks and Burnouts: Social categories and Identity in the High School. New York, NY: Teachers College Press.

Eckert, Penelope (2000). Linguistic Variation as Social Practice: The Linguistic Construction of Belten High. Malden, MA: Blackwell.

Eckert, Penelope (2008). Variation and the indexical field. Journal of Sociolinguistics, 12(4): 453-476.

Eckert, Penelope (2012). Three waves of variation study: The emergence of meaning in the study of sociolinguistic variation. Annual Review of Anthropology, 41: 87100 .

Eckert, Penelope \& Sally McConnell-Ginet (1992). Think practically and look locally: Language and gender as community-based practice. Annual Review of Anthropology, 21: 461-490.

Espy-Wilson, Carol Y, Suzanne E Boyce, Michel Jackson, Shrikanth Narayanan, Abeer Alwan (2000). Acoustic modeling of American English /r/. Journal of the Acoustical Society of America, 108(1): 343-356. 
Evanini, Keelan (2009). The permeability of dialect boundaries: A case study of the region surrounding Erie, Pennsylvania. Unpublished $\mathrm{PhD}$ thesis: University of Pennsylvania.

Evans, Linda, Alison Laurie \& Tighe Instone (2009). New Zealand homosexual law reform 1984-1986 selected chronology. In Laurie \& Evans (eds.), Twenty Years On: Histories of Homosexual Law Reform in New Zealand, pp.99-102. Wellington, NZ: LAGANZ.

Fairclough, Norman (2015). Language and Power, $3^{\text {rd }}$ ed. Abingdon, UK: Routledge. Fant, Gunnar (1960). The Acoustic Theory of Speech Production. The Hague: Mouton.

Fausto-Sterling, Anne (1993). The five sexes: Why male and female are not enough. The Sciences, 33(2): 20-25.

Fausto-Sterling, Anne (2000). Sexing the Body: Gender Politics and the Construction of Sexuality. New York: Basic Books.

Fischer, John L (1958). Social influences on the choice of a linguistic variant. Word, 14: 47-56.

Flipsen, Peter, Lawrence Shriberg, Gary Weismer, Heather Karlsson \& Jane McSweeny (1999). Acoustic characteristics of /s/ in adolescents. Journal of Speech, Language, and Hearing Research, 42: 663-677.

Forrest, Karen, Gary Weismer, Paul Milenkovic \& Ronald N. Dougall (1988). Statistical analysis of word-initial voiceless obstruents: preliminary data. Journal of the Acoustical Society of America, 84(1): 115-123).

Fought, Carmen (2003). Chicano English in Context. New York: Palgrave Macmillan.

Fox, Robert Allen \& Shawn L Nissen (2005). Sex-related acoustic changes in voiceless English fricatives. Journal of Speech, Language, and Hearing Research, 48: 753765.

Fraser, Nancy (2009). Feminism, capitalism and the cunning of history. New Left Review, 56: 97-117.

Fuchs, Susanne \& Martine Toda (2010). Do differences in male versus female /s/ reflect biological or sociophonetic factors? In Fuchs \& Toda (eds.), Interface explorations: Turbulent sounds: an interdisciplinary guide, pp.281-302. Berlin: Walter de Gruyter.

Gaudio, Rudolf P (1994). Pitch properties in the speech of gay and straight men. American Speech, 69(1): 30-57.

Geeraerts, Dirk (2008). Prototypes, stereotypes, and semantic norms. In Kristiansen \& Dirven (eds.), Cognitive Sociolinguistics: Language Variation, Cultural Models, Social Systems, pp.21-44. Berlin: Mouton de Gruyter.

Geertz, Clifford (1998). Deep hanging out. New York Review of Books, 45(16): 69. Last accessed 27 March 2017 via http://www.nybooks.com/articles/1998/10/22/deephanging-out/ 
Gelfer, Marylou Pausewang \& Quinn E Bennett (2013). Speaking fundamental frequency and vowel formant frequencies: Effects on perception of gender. Journal of Voice, 27(5): 556-566.

Gilman, Charlotte Perkins (1911). Our Androcentric Culture, or the Man Made World. Last accessed 27 March 2017 via www.gutenberg.org/cache/epub/3015/pg3015.txt

Glain, Olivier (2014). Introducing contemporary palatalisation. York Papers in LInguistics - PARLAY proceedings, Series 1: 16-29.

Gordon, Elizabeth \& Margaret Maclagan (2001). 'Capturing a sound change': a real time study over 15 years of the NEAR/SQUARE diphthong merger in New Zealand English. Australian Journal of Linguistics: 21(2): 215-238.

Gordon, Elizabeth, Lyle Campbell, Jennifer Hay, Margaret Maclagan, Andrea Sudbury \& Peter Trudgill (2004). New Zealand English: Its origins and evolution. Cambridge, UK: Cambridge University Press.

Gordon, Matthew, Paul Barthmaier \& Kathy Sands (2002). A cross-linguistic acoustic study of voiceless fricatives. Journal of the International Phonetic Association, 32(2): 141-174.

Green, David M (1976). An Introduction to Hearing. Hillsdale, NJ: Lawrence Erlbaum Associates.

Greenbaum, Sidney \& Randolph Quirk (1990). A student's grammar of the English language. Harlow UK: Longman Group.

Gumperz, John J (1962). Types of linguistic communities. Anthropological Linguistics, 4(1): 28-40.

Guy, Gregory \& Frans Hinskens (2016). Linguistic coherence: Systems, repertoires and speech communities. Lingua, 172-173: 1-9.

Gylfadottir, Duna (2015). Shtreets of Philadelphia: An acoustic study of /str/-retraction in a naturalistic speech corpus. University of Pennsylvania Working Papers in Linguistics, 21(2): 89-97.

Haley, Katrina L, Elizabeth Seelinger, Kerry Callahan Mandulak \& David J Zajac (2010). Evaluating the spectral distinction between sibilant fricatives through a speakercentered approach. Journal of Phonetics, 38: 548-554.

Harrington, Jonathan (2010). Acoustic phonetics. In Hardcastle, Laver \& Gibbon (eds.), The Handbook of Phonetic Science, 2nd ed., pp.81-129. Oxford, UK: Blackwell.

Hay, Jennifer \& Katie Drager (2010). Stuffed toys and speech perception. Linguistics, 48(4): 865-892.

Hay, Jennifer, Katie Drager \& Paul Warren (2009). Careful who you talk to: An effect of experimenter identity on the production of the NEAR/SQUARE merger in New Zealand English. Australian Journal of Linguistics, 29(2): 269-285.

Hay, Jennifer, Margaret Maclagan \& Elizabeth Gordon (2008). New Zealand English. Edinburgh: Edinburgh University Press. 
Hayward, Katrina (2000). Experimental Phonetics. Essex, UK: Pearson Education Limited.

Hazenberg, Evan (2012). Language and identity practice: A sociolinguistic study of gender in Ottawa, Ontario. Unpublished MA Thesis: Memorial University of Newfoundland.

Heffernan, Kevin (2004). Evidence from HNR that /s/ is a social marker of gender. Toronto Working Papers in Linguistics, 23(2): 71-84.

Hickey, Raymond (2014). Mergers, losses, and the spread of English. In Taavitsainen, Kytö, Claridge \& Smith (eds.), Developments in English: Expanding Electronic Evidence, pp. 237-250. Cambridge, UK: Cambridge University Press

Hillenbrand, James, Laura A Getty, Michael J Clark \& Kimberlee Wheeler (1995). Acoustic characteristicsof American English vowels. Journal of the Acoustical Society of America, 97: 3099-3111.

Hindle, Donald (1978). Approaches to vowel normalization in the study of natural speech. In Sankoff (ed.), Linguistic variation: Models and methods, pp.161-171. New York: Academic.

Hinskens, Frans \& Gregory Guy (eds.) (2016). Coherence, covariation, and bricolage: Various approaches to the systematicity of language variation. Special issue of Lingua, 172-173.

Holmes, Janet (1995). Women, Men and Politeness. London, UK \& New York: Longman.

Holmes, Janet (1996). Losing voice: is final /z/ devoicing a feature of Maori English? World Englishes, 15(2): 193-205.

Holmes, Janet \& Allan Bell (1992). On shear markets and sharing sheep: the merger of EAR and AIR diphthongs in New Zealand English. Language Variation and Change, 4: 251-273.

Holmes, Janet \& Helen Ainsworth (1996). Syllable-timing and Maori English. Te Reo, 39: $75-84$.

Holmes, Janet \& Maria Stubbe (2003). Power and Politeness in the Workplace. London, UK: Pearson Education.

Holmes, Janet \& Meredith Marra (2004). Relational practice in the workplace: Women's talk or gendered discourse? Language in Society, 33(3): 377-398.

Holmes, Janet, Meredith Marra \& Bernadette Vines (2012). Politeness and impoliteness in ethnic varieties of New Zealand English. Journal of Pragmatics, 44: 10631076.

hooks, bell (1981). ain't i a woman? black women and feminism. Boston: South End Press.

Houston, Ann Celeste (1985). Continuity and change in English Morphology: the variable (ing). Unpublished $\mathrm{PhD}$ dissertation: University of Pennsylvania. 
HRC (2008). To Be Who I Am: Report of the Inquiry into Discrimination Experienced by Transgender People. New Zealand: Human Rights Commission.

Hyman, Prue (2004). Low waged work and gender pay equity in New Zealand. Paper for the National Advisory Council on the Employment of Women Conference on Pay and Employment Equity for Women. Last accessed 27 March 2017 via http://women.govt.nz/sites/public_files/paper-low-waged-work.pdf

IBM Corp (2013). IBM and Statistics for Macintosh, Version 22.0. Armonk, NY: IBM Corp.

Ingemann, Frances (1968). Identification of the speaker's sex from voiceless fricatives. Journal of the Acoustical Society of America, 44(4): 1142-1144.

Irvine, Judith \& Susan Gal (2000). Language ideology and linguistic differentiation. In Kroskrity (ed.), Regimes of Language: Ideologies, Polities, and Identities, pp. 3583. Santa Fe, NM: School of American Research Press.

Ito, Rika \& Sali Tagliamonte (2003). Well weird, right dodgy, very strange, really cool: Layering and recycling in English intensifiers. Language in Society, 32(2): 257279.

James, Bev \& Kay Saville-Smith (1994). Gender, Culture, and Power: Challenging New Zealand's Gendered Culture, $2^{\text {nd }}$ edition. Auckland: Oxford University Press

Janda, Richard \& Brian Joseph (2003). Reconsidering the canons of sound change: Towards a "big bang" theory. In Blake \& Burridge (eds.), Historical Linguistics 2001. Selected Papers from the 15th International Conference on Historical Linguistics, Melbourne, 13-17 August 2001, pp. 205-219. Amsterdam: John Benjamins Publishing.

Jespersen, Otto (1949). A Modern English Grammar on Historical Principles. Part I: Sounds and Spellings. London, UK: George Allen \& Unwin.

Johnson, Daniel Ezra (2009). Getting off the Goldvarb standard: Introducing Rbrul for mixed-effects variable rule analysis. Language and Linguistics Compass, 3(1): 359-383.

Johnson, Keith (2003). Acoustic and Auditory Phonetics, $2^{\text {nd }}$ edition. Malden, MA: Blackwell Publishing.

Jongman, Allard, Ratree Wayland \& Serena Wong (2000). Acoustic characteristics of English fricatives. Journal of the Acoustical Society of America, 108(3): 12521263.

Jordan, Brigitte (2013). Pattern recognition in human evolution and why it matters for ethnography, anthropology, and society. In Jordan (ed.), Advancing Ethnography in Corporate Environments: Challenges and Emerging Opportunities, pp.193213. Walnut Creek, CA: Left Coast Press, Inc.

Kato, Kazuo (1986). Gradable gradability. English Studies, 67(2): 174-180.

Kedgley, Sue (1985). The Sexual Wilderness: Men and Women in New Zealand. Auckland: Reed Methuem Publishers Ltd. 
Kennedy, Christopher (1999). Projecting the Adjective: the Syntax and Semantics of Gradability and Comparison. New York, NY: Garland.

Kennedy, Marianna (2006). Variation in the Pronunciation of English by New Zealand School Children. Unpublished MA thesis. Victoria University of Wellington: Wellington, New Zealand.

Kerswill, Paul \& Ann Williams (2002). 'Salience' as an explanatory factor in language change: evidence from dialect levelling in urban England. In Jones \& Esch (eds.), Contact-induced language change: an examination of internal, external and nonlinguistic factors. Berlin: Mouton de Gruyter.

Kiesling, Scott Fabius (2005). Homosocial desire in men's talk: Balancing and recreating cultural discourses of masculinity. Language in Society, 34: 695-726.

King, Michael (2003). The Penguin History of New Zealand. Auckland, NZ: Penguin Books.

Labov, William (1963). The social motivation of a sound change. Word, 19: 273-309. ALSO chapter 1 of Labov (1972): Sociolinguistic Patterns. Philadelphia: University of Philadelphia Press.

Labov, William (1966). The Social Stratification of English in New York City. Washington, DC: Center for Applied Linguistics.

Labov, William (1972). Sociolinguistic patterns. Philadelphia: University of Pennsylvania Press.

Labov, William (1984). Field methods of the Project on Language Change and Variation. In Baugh \& Shezer (eds.), Language Use, pp.28-53. Englewood Cliffs, NJ: Prentice Hall.

Labov, William (1990). The intersection of sex and social class in the course of linguistic change. Language Variation and Change, 2(2): 205-254.

Labov, William (1994). Principles of Linguistic Change. Volume 1: Internal Factors. Oxford, UK: Blackwell.

Labov, William (2001). Principles of Linguistic Change, Volume 2: Social factors. Oxford, UK: Blackwell.

Labov, William, Malcah Yaeger \& Richard Steiner (1972). A Quantitative Study of Sound Change in Progress. Philadelphia, PA: US Regional Survey.

Labov, William, Sharon Ash \& Charles Boberg (2006). Atlas of North American English: Phonetics, Phonology and Sound Change. Berlin \& New York: Mouton de Gruyter.

Ladefoged, Peter \& Ian Maddieson (1996). The Sounds of the World's Languages. Oxford, UK \& Cambridge, MA: Blackwell Publishers.

Langstrof, Christian (2006). Vowel change in New Zealand English-patterns and implications. PhD Thesis: University of Canterbury.

Language Archive, The (2015). ELAN Programme Suite, version 4.9.2. Max Planck Institute for Psycholinguistics, Nijmegen: http://tla.mpi.nl/tools/tla-tools/elan/ 
Laurie, Alison J \& Linda Evans (2009). Legislating homosexuality. In Laurie \& Evans (eds.), Twenty Years On: Histories of Homosexual Law Reform in New Zealand, pp.95-97. Wellington, NZ: LAGANZ.

Laurie, Alison J \& Linda Evans (eds.) (2009a). Twenty Years On: Histories of Homosexual Law Reform in New Zealand. Wellington, NZ: LAGANZ.

Lave, Jean \& Etienne Wenger (1991). Situated Learning: Legitimate Peripheral Participation. Cambridge, UK: Cambridge University Press.

Lawrence, Wayne P (2000). /str/ $\longrightarrow$ / $\mathrm{ftr} /:$ Assimilation at a distance? American Speech, 75(1): 82-87.

Le Page, Robert Brock \& Andrée Tabouret-Keller (1985). Acts of Identity: CreoleBased Approaches to Language and Ethnicity. Cambridge, UK: Cambridge University Press.

Levon, Erez (2015). Integrating intersectionality in language, gender, and sexuality research. Language and Linguistics Compass, 9(7): 295-308.

Levon, Erez \& Sophie Holmes-Elliott (2013). East end boys and west end girls: /s/fronting in Southeast England. University of Pennsylvania Working Papers in Linguistics, 19(2): 111-120.

Linville, Sue Ellen (1998). Acoustic correlates of perceived versus actual sexual orientation in men's speech. Folia Phoniatrica et Logopaedica, 50: 35-48.

Llamas, Carmen (2012). Scottish, English, British? Coding for attitude in the UK. Paper presented at LSA 2012, Satellite workshop for Sociolinguistic Archival Preparation. Portland, OR.

Llamas, Carmen, Dominic Watt \& Andrew E MacFarlane (2016). Estimating the relative sociolinguistic salience of segmental variables in a dialect boundary zone. Frontiers in Psychology, 7: 1163. DOI: 10.3389/fpsyg.2016.01163

Lobanov, Boris M (1971). Classification of Russian vowels spoken by different listeners. Journal of the Acoustical Society of America, 49(2): 606-608.

Macdonald, Charlotte (1993). The Vote, The Pill, and the Demon Drink: A History of Feminist Writing in New Zealand 1869-1993. Wellington: Bridget Williams Books.

Maclagan, Margaret (1982). An acoustic study of New Zealand vowels. The New Zealand Speech Therapists' Journal, 37(1): 20-26.

Maclagan, Margaret \& Elizabeth Gordon (1996). Out of the AIR and into the EAR: another view of the New Zealand diphthong merger. Language Variation and Change, 8: 125-147.

Maclagan, Margaret \& Jen Hay (2004). The rise and rise of New Zealand English DRESS. Proceedings of the 10th Australian International Conference on Speech Science \& Technology, pp. 183-188. Macquarie University, Sydney: 8-10 December. 
Maclagan, Margaret \& Jennifer Hay (2007). Getting fed up with our feet: Contrast maintenance and the New Zealand English "short" front vowel shift. Language Variation and Change, 19: 1-25.

Maclagan, Margaret, Elizabeth Gordon \& Gillian Lewis (1999). Women and sound change: Conservative and innovative behavior by the same speakers. Language Variation and Change, 11: 19-41.

Mægaard, Marie \& Nicolai Pharao (2016). /s/ variation and perceptions of male sexuality in Denmark. In Levon and Mendes (eds.), Language, Sexuality, and Power, pp.33-104. New York: Oxford University Press.

Maniwa, Kazumi, Allard Jongman \& Travis Wade (2009). Acoustic characteristics of clearly spoken English fricatives. Journal of the Acoustical Society of America, 125(6): 3962-3973.

Marriage (Definition of Marriage) Amendment Act 2013. Last accessed 27 March 2017 via http://www.legislation.govt.nz/act/public/2013/0020/latest/DLM4505003.html

Marsden, Sharon (2013). Phonological variation and the construction of regional identities in New Zealand English. Unpublished PhD thesis, Victoria University of Wellington. Wellington, New Zealand.

Mattson, Mark P (2014). Superior pattern processing is the essence of the evolved human brain. Frontiers in Neuroscience, 8: 265. Last accessed 27 March 2017 via https://www.ncbi.nlm.nih.gov/pmc/articles/PMC4141622/

McKenzie, Jayne (2005). "But he's not supposed to see me in my weeding dress!" - The relationship between DRESS and FLEECE in modern New Zealand English. New Zealand English Journal, 19: 13-25.

Meyerhoff, Miriam (2001). Dynamics of differentiation: On social psychology and cases of language variation. In Coupland, Sarangi \& Candlin (eds.), Sociolinguistics and Social Theory, pp.61-97. Harlow, UK: Pearson Education.

Meyerhoff, Miriam (2015). Turning variation on its head: Analysing subject prefixes in Nkep (Vanuatu) for language documentation. Asia-Pacific Language Variation, 1(1): 78-108.

Meyerhoff, Miriam \& James N Stanford (2015). "Tings change, all tings change": The changing face of sociolinguistics with a global perspective. In Smakman \& Heinrich (eds.), Globalising Sociolinguistics, pp. 1-15. Abingdon, UK: Routledge.

Meyerhoff, Miriam \& Steffen Klaere (2017). A case for clustering speakers and linguistic variables: Big issues with smaller samples in language variation. In Buchstaller \& Siebenhaar (eds.), Language Variation - European Perspectives VI, pp. 23-46. Amsterda: John Benjamins.

Miller, James D (1989). Auditory-perceptual interpretation of the vowel. Journal of the Acoustical Society of America, 85(5): 2114-2134.

Milroy, James \& Lesley Milroy (1985). Linguistic change, social network and speaker innovation. Journal of Linguistics, 21: 339-384. 
Ministry for Women (2016). Gender pay gap. Last accessed 27 March 2017 via http://women.govt.nz/work-skills/income/gender-pay-gap

Munson, Benjamin \& Alexander Coyne (2010). The influence of apparent vocal-tract size, contrast type, and implied sources of variation on the perception of American English voiceless lingual fricatives. Journal of the Phonetic Society of Japan, 14(1): 35-47.

Munson, Benjamin \& Lindsey J. Zimmerman (2006). Perceptual bias and the myth of the gay lisp. Poster presentation at American Speech-Language Hearing Association conference: Miami, FL. Last accessed 27 March 2017 via http://www.asha.org/Events/convention/handouts/2006/1165_Munson_Benjamin/

Munson, Benjamin, Elizabeth C McDonald, Nancy L DeBoe \& Aubrey R White (2006). The acoustic and perceptual bases of judgments of women and men's sexual orientation from read speech. Journal of Phonetics, 34: 2002-240.

Munson, Benjamin, Sarah Jefferson \& Elizabeth McDonald (2006). The influence of perceived sexual orientation on fricative identification. Journal of the Acoustical Society of America, 119(4): 2427-2437.

Mustanoja, Tauno (1960). A Middle English Syntax. Helsinki: Société Néophilogique.

Nearey, Terrence M (1977). Phonetic feature systems for vowels. Unpublished PhD dissertation. The University of Alberta. Reprinted 1978 by the Indiana University Linguistics Club. Last accessed 27 March 2017 via http://www.ualberta.ca/ tnearey/Nearey1978_compressed.pdf

Newman, Rochelle S, Sheryl A Clouse \& Jessica L Burnham (2001). The perceptual consequences of within-talker variability in fricative production. Journal of the Acoustical Society of America, 109(3): 1181-1196.

Nittrouer, Susan (1995). Children learn separate aspects of speech production at different rates: evidence from spectral moments. Journal of the Acoustical Society of America, 97(1): 520-530.

Nolan, Melanie (2007). The reality and myth of New Zealand Egalitarianism: Explaining the pattern of a labour historiography at the edge of empires. Labour History Review, 72(2): 113-134.

Nunberg, Geoffrey (1980). A falsely reported merger in eighteenth century English: a study in diachronic variation. In Labov (ed.), Locating language in time and space, pp.221-50. New York: Academic Press.

O’Connor, Sean (1998). Straight Acting: Popular Gay Drama from Wilde to Rattigan. Washington DC: Cassell.

O’Donnell, Michael (2008a). The UAM CorpusTool: Software for corpus annotation and exploration. Proceedings of the XXVI Congreso de AELSA. Almeria, Spain.

O’Donnell, Michael (2008b). UAMCorpusTool 3.1.14. http://www.wagsoft.com/CorpusTool/ 
Ochs, Elinor (1990). Indexicality and socialization. In Stigler, Shweder \& Herdt (eds.), Cultural Psychology: Essays on Comparative Human Development, pp. 287-308. Cambridge, UK: Cambridge University Press.

Ochs, Elinor (1992). Indexing gender. In Duranti \& Goodwin (eds.), Rethinking Context: Language as an Interactive Phenomenon, pp.335-358. Cambridge: Cambridge University Press.

Oswin, Natalie (2006). Decentering queer globalization: diffusion and the 'global gay'. Environment and Planning D: Society and Space, 24: 777-790.

Paolillo, John C (n.d.). ALV R-Varb. Last accessed 27 March 2017 via http://paolillo.soic.indiana.edu/index.php/ALV/RVarb

Papp, Viktória (2011). The female-to-male transsexual voice: Physiology vs. performance in production. Unpublished $\mathrm{PhD}$ thesis, Rice University. Houston, Texas.

Parkinson, Phil (2009). Legitimation through disaster? AIDS and the homosexual law reform campaign, 1983-1993. In Laurie \& Evans (eds.), Twenty Years On:

Histories of Homosexual Law Reform in New Zealand, pp.39-48. Wellington, NZ: LAGANZ.

Partington, Alan (1993). Corpus evidence of language change: The case of intensifiers. In Baker et al (eds.), Text and Technology: In Honour of John Sinclair, pp.177192. Amsterdam \& Philadelphia: John Benjamins.

Peters, Hans (1994). Degree adverbs in early modern English. In Kastovksy (ed.), Studies in Early Modern English, pp. 269-288. Berlin \& New York: Walter de Gruyter.

Peterson, Gordon E \& Harold L Barney (1952). Control methods used in a study of the vowels. Journal of the Acoustical Society of America, 24(2): 175-184.

Petrucci, Peter R. \& Michael Head (2006). Sweet as is cool for New Zealanders. American Speech, 81(3): 331-336.

Pharao, Nicolai, Marie Maegaard, Janus Spindler Møller \& Tore Kristiansen (2014). Indexical meanings of $\left[\mathrm{s}^{+}\right]$among Copenhagen youth: Social perception of a phonetic variant in different prosodic contexts. Language in Society, 43: 1-31.

Phillips, Jock (1996). A Man's Country? The Image of the Pakeha Male: A History. Auckland: Penguin Books.

Plomp, Reinier (1976). Aspects of Tone Sensation: a Psychophysical Study. London: Academic Press.

Plomp, Reinier (2002). The Intelligent Ear: On the Nature of Sound Perception. Hillsdale, NJ: Lawrence Erlbaum Associates.

Podesva, Rob \& Janneke Van Hofwegen (2016). /s/exuality smalltown California. In Levon \& Mendes (eds.), Language, Sexuality, and Power, pp. 168-187. New York: Oxford University Press. 
Podesva, Robert J (2007). Phonation type as stylistic variable: The use of falsetto in constructing a persona. Journal of Sociolinguistics, 11(4): 478-504.

Podesva, Robert J (2008). Three sources of stylistic meaning. Texas Linguistic Forum, 51: 134-143.

Podesva, Robert J (2011). Salience and the social meaning of declarative contours: Three case studies of gay professionals. Journal of English Linguistics, 39(3): 233-264.

Press, William H, Brian P Flannery, Saul A Teukolsky \& William T Vetterling (1988). Numerical Recipes in C: The Art of Scientific Computing. Cambridge, UK: Cambridge University Press.

Pullum, Geoffrey K. \& Rodney Huddleston (2002). In Huddleston \& Pullum (eds.), The Cambridge Grammar of the English Language, pp. 525-596. Cambridge UK: Cambridge University Press.

Quirk, Randolph, Sidney Greenbaum, Geoffrey Leach \& Jan Svartvik (1973). A Grammar of Contemporary English, 2nd ed. London, UK: Longman.

Quirk, Randolph, Sidney Greenbaum, Geoffrey Leach \& Jan Svartvik (1985). A Comprehensive Grammar of the English Language. New York: Longman.

R Core Team (2013). R: A language and environment for statistical computing. $\mathrm{R}$ Foundation for Statistical Computing, Vienna, Austria. ISBN 3-900051-07-0. http://www.R-project.org/

Rampton, Ben (1995). Crossing: Language and Ethnicity Among Adolescents. Essex, UK: Longman.

Rampton, Ben (1999). Crossing. Journal of Linguistic Anthropology, 9(1-2): 54-56.

Rendall, Drew, Paul L Vasey \& Jared McKenzie (2008). The queen's English: an alternative, biosocial hypothesis for the distinctive features of "gay speech". Archives of Sexual Behavior, 37(1): 188-204.

Rickford, John R (1987). The haves and have-nots: Sociolinguistic surveys and the assessment of speaker competence. Language in Society, 16(2): 149-177.

Risberg, Gunilla, Eva E Johansson \& Katrina Hamberg (2009). A theoretical model for analysing gender bias in medicine. International Journal for Equity in Health, 8(28). DOI:10.1186/1475-9276-8-28

Robertson, Stuart (1954). The Development of Modern English. Rev. by Frederic G Cassidy. Englewood Cliffs, NJ: Prentice-Hall.

Rosch, Eleanor (1973). Natural categories. Cognitive Psychology, 4(3): 328-350.

Rosch, Eleanor (1978). Principles of categorization. In Rosch \& Lloyd (eds.), Cognition and Categorization, pp.27-48. Hillsdale, NJ: Lawrence Erlbaum Associates.

Rosenfelder, Ingrid, Joe Fruehwald, Keelan Evanini \& Jiahong Yuan (2011). FAVE (Forced Alignment and Vowel Extraction) Program Suite. http://fave.ling.upenn.edu 
Rosenfelder, Ingrid, Joe Fruehwald, Keelan Evanini \& Jiahong Yuan (2011a). FAVEextract-options. Last accessed 27 March 2017 via http://fave.ling.upenn.edu/usingEF.html

Rosenfelder, Ingrid, Joe Fruehwald, Keelan Evanini \& Jiahong Yuan (2011b). How to use FAVE-extract. Last accessed 27 March 2017 via http://fave.ling.upenn.edu/usingEF.html

Saarenpää, Tuomas (2016). A comparative corpus study on intensifiers in Canadian English and New Zealand English. Unpublished MA Thesis: University of Tampere, Finland. Last accessed 27 March 2017 via https://tampub.uta.fi/bitstream/handle/10024/98915/GRADU1462354862.pdf?sequence $=1$

Sankoff, David (1975). VARBRUL version 2. Unpublished program and documentation.

Sankoff, David (1988). Variable rules. In Ammon, Dittmar \& Mathheier (eds.), Berlin Sociolinguistics: An International Handbook of the Science of Language and Society, Vol. 2, pp. 984-997. Berlin: Walter de Gruyter.

Sankoff, David, Sali Tagliamonte \& Eric Smith (2005). Goldvarb X: A variable rule application for Macintosh and Windows. Department of Linguistics, University of Toronto. Last accessed 27 March 2017 via http://individual.utoronto.ca/tagliamonte/goldvarb.html

Scandrett, Gina (2012). Intensifiers in New Zealand English. New Zealand English Journal, 26: 33-42.

Schiel, Florian (1999). Automatic Phonetic Transcription of Non-Prompted Speech. Proceedings of the International Conference on Phonetic Sciences (ICPhS) August 1999: 607-610.

Schmid, Helmut (1994). Probabilistic Part-of-Speech tagging using decision trees. Proceedings of the International Conference on New Methods in Language Processing. Manchester, UK. Last accessed 27 March 2017 via http://www.cis.uni-muenchen.de/ schmid/tools/TreeTagger/data/tree-tagger1.pdf

Schwartz, Martin F. (1968). Identification of speaker sex from isolated, voiceless fricatives. Journal of the Acoustical Society of America, 43(5): 1178-1179.

Shadle, Christine Helen (1985). The Acoustics of Fricative Consonants. Cambridge, MA: MIT.

Shadle, Christine Helen (1991). The effect of geometry on source mechanisms of fricative consonants. Journal of Phonetics, 19: 409-424.

Shapiro, Michael (1995). A case of distant assimilation: /str/ -->/ftr/. American Speech, 70(1): 101-107.

Shermer, Michael (2008). Patternicity: Finding meaningful patterns in meaningless noise. Scientific American. Last accessed 27 March 2017 via https://www.scientificamerican.com/article/patternicity-finding-meaningfulpatterns/ 
Silverstein, Michael (1985). Language and the culture of gender: At the intersection of structure, usage and ideology. In Mertz \& Parmentier (eds.), Semiotic Mediation: Sociocultural and Psychological Perspectives, pp. 219-259. New York: Academic Press.

Silverstein, Michael (2003). Indexical order and the dialectics of sociolinguistic life. Language \& Communication, 23: 193-229.

Simpson, Tony (2016). More tolerant than you might think. Stout Research Centre Seminar. Victoria University of Wellington, NZ: 2 November.

Skilling, Peter (2013). Egalitarian myths in New Zealand: a review of public opinion data on inequality and redistribution. New Zealand Sociology, 28(2): 16-43.

SLALS (n.d.). The Wellington Corpus of Spoken English. Last accessed 27 March 2017 via http://www.victoria.ac.nz/lals/resources/corpora-default/corpora-wsc

Sloetjes, H \& P Wittenburg (2008). Annotation by category - ELAN and ISO DCR. Proceedings of the 6th International Conference on Language Resources and Evaluation (LREC 2008).

Smyth, Ron \& Henry Rogers (2008). Do gay-sounding men speak like women? Toronto Working Papers in Linguistics, 27: 129-144.

Sowa, Joseph (2009). 'Sweet as!': the intensifier as in New Zealand and Australian English. English Today, 25(2): 58-61.

Spivak, Gayatri Chakravorty (1984-85). Criticism, feminism and the institution. Thesis Eleven, 10-11: 175-187.

Spivak, Gayatri Chakravorty (1990). The Post-Colonial Critic: Interviews, Strategies, Dialogues. New York: Routledge.

Starks, Donna (2000). Distinct, but not too distinct: gender and ethnicity as determinants of (s) fronting in four Auckland communities. English World-Wide, 21(2): 291304.

Statistics New Zealand (2016). Infoshare. Last accessed 27 March 2017 via http://www.stats.govt.nz/infoshare/

Stevens, Kenneth (1989). On the quantal nature of speech. Journal of Phonetics, 17: 345.

Stevens, Kenneth (1998). Acoustic Phonetics. Cambridge: MIT Press.

Stevens, Stanley Smith, John Volkmann \& Edwin B. Newman (1937). A scale for the measurement of the psychological magnitude pitch. Journal of the Acoustical Society of America, 8(3): 189-190.

Stoffel, Cornelis (1901). Intensives and Down-Toners. Heidelberg: Carl Winter.

Strand, Elizabeth (1999). Uncovering the role of gender stereotypes in speech perception. Journal of Language and Social Psychology, 18: 86-99.

Stryker, Susan (2008). Transgender History. Berkeley, CA: Seal Press. 
Stuart-Smith, Jane (2007). Empirical evidence for gendered speech production: /s/ in Glaswegian. In Coates \& Hualde (eds.), Laboratory Phonology 9, pp.65-86. New York: Mouton de Gruyter.

Tabain, Marija (2001). Variability in fricative production and spectra: implications for the hyper- and hypo- and quantal theories of speech production. Language and Speech, 44(1): 57-94.

Tagliamonte, Sali (2006). Analysing Sociolinguistic Variation. New York, NY \& Cambridge, UK: Cambridge University Press.

Tagliamonte, Sali (2008). So different and pretty cool! Recycling intensifiers in Toronto, Canada. English Language and Linguistics, 12(2): 361-394.

Tagliamonte, Sali \& Chris Roberts (2005). So weird; so cool; so innovative: The use of intensifiers in the television series Friends. American Speech, 80(3): 280-300.

Tagliamonte, Sali \& Dylan Uscher (2009). Queer youth in the speech community: Enriching large scale studies of variation and change. Paper presented at NWAV 38. University of Ottawa: 22-25 October.

Taylor, Ben (1998). Exploring the "gay accent": Features of the speech of gay men in Wellington. Unpublished MA Thesis, Victoria University of Wellington. Wellington, New Zealand.

Thomas, Erik \& Tyler Kendall (2015a). About vowel normalization. Accessed 19 March 2016 via http://lingtools.uoregon.edu/norm/about_normalization1.php

Thomas, Erik \& Tyler Kendall (2015b). NORM's vowel normalization methods (v. 1.1). Last accessed 27 March 2017 via http://lingtools.uoregon.edu/norm/norm1_methods.php

Thomas, Erik R (2011). Sociophonetics: An Introduction. New York: Palgrave Macmillan.

Toutanova, Kristina, Dan Klein, Christopher Manning \& Yoram Singer (2003). Featurerich part-of-speech tagging with a cyclic dependency network. Proceedings of HLT-NAACL 2003, pp. 252-259. Last accessed 27 March 2017 via http://nlp.stanford.edu/ manning/papers/tagging.pdf

Trudgill, Peter (1974). The Social Differentiation of English in Norwich. Cambridge, UK: Cambridge University Press.

Trudgill, Peter (1986). Dialects in Contact. Oxford, UK: Blackwell.

van Bezooijen, Renée \& Vincent J. van Heuven (2010). Avant-garde Dutch: A perceptual, acoustic, and evaluational study. In Preston \& Niezdzielski (eds.), $A$ Reader in Sociophonetics, pp. 357-378. New York, NY: Walter de Gruyter.

Verdonk, Petra, Yvonne WM Benschop, Hanneke CJM de Haes, Toine LM LagroJanssen (2009). From gender bias to gender awareness in medical education. Advances in Health Science Education, 14: 135-152.

Visser, Irene (1996). The prototypicality of gender: Contemporary notions of masculine and feminine. Women's Studies International Forum, 19(6): 589-600. 
Visser, Irene (2002). Prototypes of gender: Conceptions of masculine and feminine. Women's Studies International Forum, 25(5): 529-539.

Walker, James A (2010). Variation in Linguistic Systems. New York, NY: Routledge.

Warren, Paul (2004). On the front foot: the quality and quantity of ANZE vowels. Paper presented at PW PANZE, Sydney: December 8.

Warren, Paul (2005). Word recognition and sound merger. In Luchjenbroers (ed.), Cognitive Linguistic Investigations, pp. 169-186. Amsterdam \& Philadelphia: John Benjamins.

Warren, Paul (2006a). Oops, I've done a futt: Quality and quantity in a New Zealand vowel contrast. Te Reo, 49: 125-143.

Warren, Paul, Megan Rae \& Jen Hay (2003). Word recognition and sound merger: the case of the front-centering diphthongs in NZ English. In Solé, Recasens \& Romero (eds.), Proceedings of the 15th International Congress of Phonetic Sciences, pp. 2989-2992. Rundle Mall: Causal Productions.

Watson, Catherine \& Jonathan Harrington (1999). Acoustic evidence for dynamic formant trajectories in Australian English vowels. Journal of the Acoustical Society of America, 106(1): 458-468.

Watson, Catherine, Jonathan Harrington \& Zoe Evans (1998). An acoustic comparison between New Zealand and Australian English vowels. Australian Journal of Linguistics, 18(2): 185-207.

Watson, Catherine, Margaret Maclagan \& Jonathan Harrington (2000). Acoustic evidence for vowel change in New Zealand English. Language Variation and Change, 12: 51-68.

Watson, Catherine, Margaret Maclagan, Jeanette King, Ray Harlow, \& Peter Keegan (2016). Sound change in Māori and the Influence of New Zealand English. Journal of the International Phonetics Association, 46(2): 185-218.

Weinreich, Uriel, William Labov \& Marvin Herzog (1968). Empirical foundations for a theory of language change. In Lehman \& Malkiel (eds.), Directions for Historical Linguistics, pp. 95-188. Austin, TX: University of Texas Press.

Wells, John Christopher (1982). Accents of English 3: Beyond the British Isles. Cambridge, UK: Cambridge University Press.

Wetherell, Margaret (1998). Positioning and interpretative repertoires: Conversation analysis and post-structuralism in dialogue. Discourse \& Society, 9(3): 387-412.

Whiteside, Sandra P (1996). Temporal-based acoustic-phonetic patterns in read speech: Some evidence for speaker sex differences. Journal of the International Phonetic Association, 26(1): 23-40.

Williams, Briony (2008). EMU Speech Database system [review]. Language Documentation \& Conservation, 2(1): 166-175.

Williamson, Maurice (2013). Speech to New Zealand Parliament. Last accessed 27 March 2017 via https://www.youtube.com/watch?v=VRQXQxadyps 
Winkelman, Raphael, Klaus Jaensh, Steve Cassidy \& Jonathan Harrington (2016). Main package of the EMU Speech Database Management System. https://github.com/IPS-LMU/emuR

Wolfram, Walter A (1969). A Sociolinguistic Description of Detroit Negro Speech. Washington, DC: Center for Applied Linguistics.

Woods, Howard B (1999). The Ottawa survey of Canadian English. Strathy Language Unit occasional papers, 4. Kingston, Ontario: Queen's University.

Wright, Matthew (2013). Illustrated History of New Zealand. Auckland, NZ: David Bateman.

Wright, Richard \& David Nichols (2014). Measuring vowel formants. Last accessed 27 March 2017 via https://depts.washington.edu/phonlab/resources/measuringformants.pdf

Zhang, Qing (2005). A Chinese yuppie in Beijing: Phonological variation and the construction of a new professional identity. Language in Society, 34(3): 431-466.

Zhang, Qing (2008). Rhotacization and the 'Beijing Smooth Operator': the social meaning of a linguistic variable. Journal of Sociolinguistics, 12(2): 201-222.

Zimman, Lal (2012). Voices in transition: testosterone, transmasculinity, and the gendered voice among female-to-male transgender people. Unpublished $\mathrm{PhD}$ dissertation. University of Colorado: Boulder, Colorado.

Zimman, Lal (2013). Hegemonic masculinities and the variability of gay-sounding speech. Journal of Language and Sexuality, 2(1): 1-39.

Zsiga, Elizabeth C (2013). The Sounds of Language: An Introduction to Phonetics and Phonology. Malden, MA: Wiley-Blackwell.

Zwicker, Eberhard (1961). Subdivision of the audible frequency range into critical bands. Journal of the Acoustical Society of America, 33(2): 248.

Zwicker, Eberhard and Hugo Fastl (1990). Psychoacoustics: Facts and Models. Berlin: Springer-Verlag.

Zwicky, Arnold M (1997). Two lavender issues for linguistics. In Livia \& Hall (eds.), Queerly Phrased: Language, Gender, and Sexuality, pp.21-34. Oxford: Oxford University Press. 


\section{Appendix A}

THE INTERNATIONAL PHONETIC ALPHABET (revised to 2005)

\begin{tabular}{|c|c|c|c|c|c|c|c|c|c|c|c|c|}
\hline \multicolumn{13}{|c|}{ CONSONANTS (PULMONIC) } \\
\hline & Bilabial & Labiodental & Dental & Alveolar & Postalveolar & Retroflex & Palatal & Vela & & Uvular & Pharyngeal & Glottal \\
\hline Plosive & $\mathrm{p} \quad \mathrm{b}$ & & & $\mathrm{t} d$ & & $\mathrm{~d} d$ & c $\mathrm{f}$ & & g & $q \mathrm{G}$ & & $?$ \\
\hline Nasal & $\mathrm{m}$ & $\mathrm{m}$ & & $\mathrm{n}$ & & $\eta$ & $\mathrm{j}$ & & $\eta$ & $\mathrm{N}$ & & \\
\hline Trill & B & & & $\mathrm{r}$ & & & & & & $\mathbf{R}$ & & \\
\hline Tap or Flap & & $r$ & & ؟ & & [ & & & & & & \\
\hline Fricative & $\phi \beta$ & $\mathrm{V}$ & $\theta \quad$ ठ & S Z & 3 & S $\quad \mathrm{Z}$ & Ç J & $\mathrm{X}$ & $\gamma$ & $\chi \quad$ в & $\hbar \mathrm{S}$ & h $\mathrm{h}$ \\
\hline $\begin{array}{l}\text { Lateral } \\
\text { fricative }\end{array}$ & & & & 13 & & & & & & & & \\
\hline Approximant & & $v$ & & I & & I & $\mathrm{j}$ & & Щ & & & \\
\hline $\begin{array}{l}\text { Lateral } \\
\text { approximant }\end{array}$ & & & & 1 & & l & $\Lambda$ & & L & & & \\
\hline
\end{tabular}

Where symbols appear in pairs, the one to the right represents a voiced consonant. Shaded areas denote articulations judged impossible.

CONSONANTS (NON-PULMONIC)

\begin{tabular}{|c|c|c|c|c|}
\hline \multicolumn{2}{|r|}{ Clicks } & Voiced implosives & \multicolumn{2}{|r|}{ Ejectives } \\
\hline$\odot$ & Bilabial & 6 Bilabial & & Examples: \\
\hline & Dental & d Dental/alveolar & $\mathrm{p}^{\prime}$ & Bilabial \\
\hline$!$ & (Post)alveolar & $f$ Palatal & $t^{\prime}$ & Dental/alveolar \\
\hline$\neq$ & Palatoalveolar & d velar & $\mathrm{k}^{\prime}$ & Velar \\
\hline$\|$ & Alveolar lateral & $G^{\prime}$ Uvular & $\mathrm{s}^{\prime}$ & Alveolar fricative \\
\hline
\end{tabular}

VOWELS

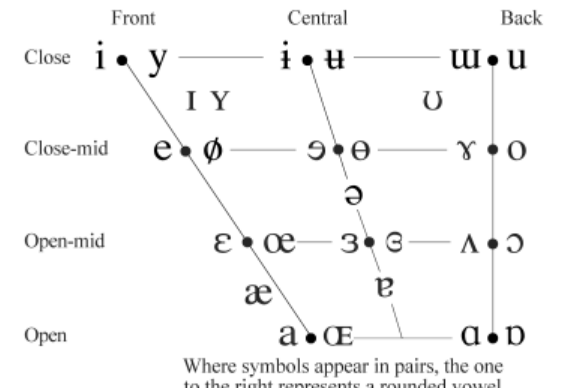

OTHER SYMBOLS

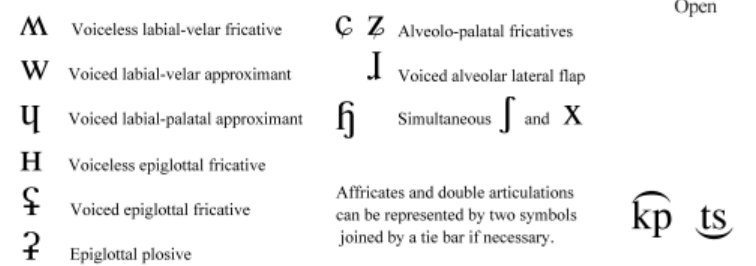

DIACRITICS Diacritics may be placed above a symbol with a descender, e.g. $\stackrel{\circ}{7}$

\begin{tabular}{|c|c|c|c|c|c|}
\hline & Voiceless & $n_{0} \mathrm{~d}_{0}$ & .. & Breathy voiced $\quad$ b & $\underset{n}{\mathrm{t}} \underset{n}{\mathrm{~d}}$ \\
\hline & Voiced & $S t$ & $\sim$ & Creaky voiced $\quad \underset{\sim}{\mathrm{a}} \underset{\sim}{\mathrm{a}}$ & $\mathrm{t} d$ \\
\hline $\mathrm{h}$ & Aspirated & $\mathrm{t}^{\mathrm{h}} \mathrm{d}^{\mathrm{h}}$ & - & Linguolabial $\quad \stackrel{\mathrm{t}}{\mathrm{d}}$ & $\mathrm{t} d$ \\
\hline , & More rounded & วิ & w & $\mathrm{t}^{\mathrm{W}} \mathrm{d}^{\mathrm{w}}$ & Nasalized \\
\hline$c$ & Less rounded & $\rho_{c}$ & $\mathrm{j}$ & Palatalized $\quad \mathrm{t}^{\mathrm{j}} \quad \mathrm{d}^{\mathrm{j}}$ & Nasal release \\
\hline+ & Advanced & $\mathbf{u}_{+}$ & $\gamma$ & Velarized $\quad t^{Y} d^{X}$ & 1 Lateral release \\
\hline _ & Retracted & $\underline{\mathrm{e}}$ & S & Pharyngealized $\mathrm{t}^{\mathrm{S}} \mathrm{d}^{\mathrm{S}}$ & \urcorner$\left._{\text {No audible release }} \quad \mathrm{d}\right\urcorner$ \\
\hline .. & Centralized & $\ddot{\mathrm{e}}$ & $\sim$ & velarized or pharyngealized $\mathbf{f}$ & \\
\hline$x$ & Mid-centralized & $\stackrel{x}{e}$ & $\perp$ & Raised & $=$ voiced alveolar fricative) \\
\hline 1 & Syllabic & $n$ & $T$ & Lowered & $=$ voiced bilabial approximant $)$ \\
\hline n & Non-syllabic & en & t & Advanced Tongue Root & \\
\hline 2 & Rhoticity & $\partial^{2} \mathrm{a}^{2}$ & t & Retracted Tongue Root & \\
\hline
\end{tabular}

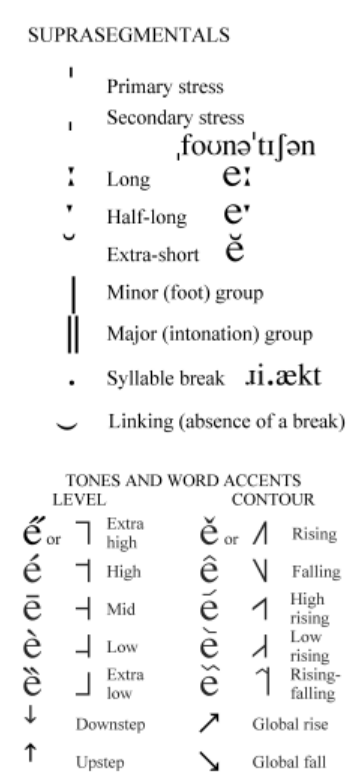

IPA Chart, http://www.internationalphoneticassociation.org/content/ipa-chart, available under a Creative Commons Attribution-Sharealike 3.0 Unported License. Copyright (C) 2015 International Phonetic Association. 


\section{Appendix B}

\section{Praat script for sibilant identification and measurement}

(particular thanks to Dr Sasha Calhoun for help with this)

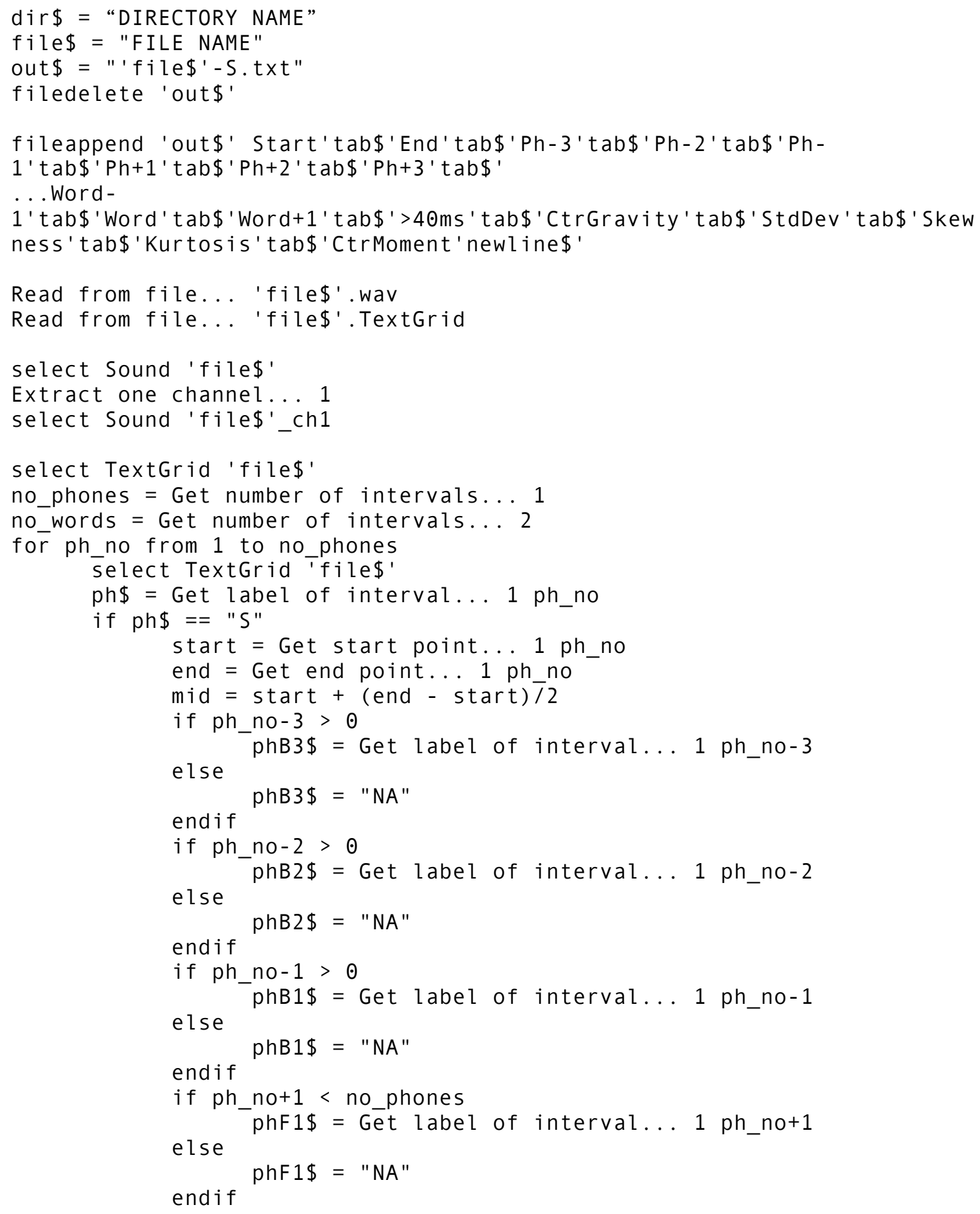




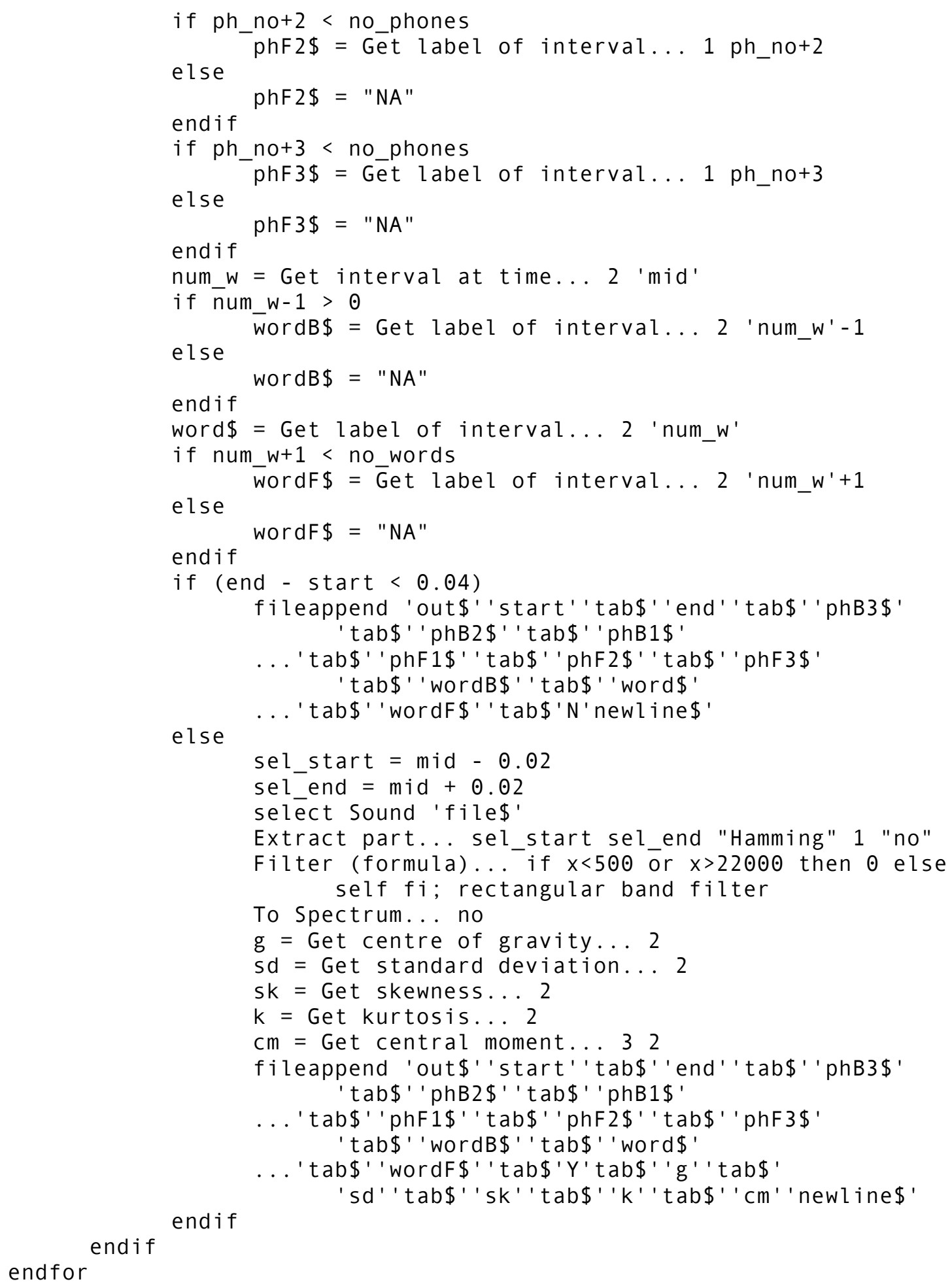




\section{Appendix C}

\section{URLs for blogs discussing (str) retraction, i.e. /st../ $\rightarrow$ [ft..]}

Note: all URLs current as of March 27, 2017

Antimoon (original post 2008). http://www.antimoon.com/forum/t10923.htm

English Forums (original post 2012).

https://www.englishforums.com/English/SoundBecomingFrequent/bbvwmh/post. htm

Grammar Girl (original post 2016).

http://www.quickanddirtytips.com/education/grammar/how-s-backing-causespeople-to-pronounce-street-as-schtreet

Literal-Minded (original post 2011).

https:/literalminded.wordpress.com/2011/09/06/shtraight-talk/

Locally Grown (original post 2011). locallygrownnorthfield.org/post/24221

Mr Verb (orignial post 2008). http://mr-verb.blogspot.co.nz/2008/08/michelle-obamasstr-tr.html

Pain in the English (original post 2014). http://painintheenglish.com/case/5231

The Chicago Tribune (original story 6 October 2011).

http://blogs.chicagotribune.com/news_columnists_ezorn/2011/10/shtay-shtrongshtraight-talkers-youre-not-so-shtrange.html

The Free Dictionary (original post 2010).

http://forum.thefreedictionary.com/postst9655_The-Phantom--H-.aspx

The Straight Dope (original post 2010).

http://boards.straightdope.com/sdmb/showthread.php?t=548320 


\section{Appendix D}

\section{Means for vowel measurements}

For each vowel measured, the following tables provide the number of tokens and the means for each gendered identity group:

- F1 and F2 for monophthongs

- F1 and F2 of onsets and offglides for diphthongs

- Euclidean Distance (ED) as relevant (calculated as discussed in §6.2.6.1)

The tables are ordered alphabetically by lexical set.

\begin{tabular}{|c|c|c|c|c|c|}
\hline \multicolumn{6}{|c|}{ CHOICE } \\
\hline Group & $\mathbf{N}$ & $\begin{array}{c}\text { F1 onset } \\
(\mathrm{Hz})\end{array}$ & $\begin{array}{c}\text { F2 onset } \\
(\mathrm{Hz})\end{array}$ & $\begin{array}{c}\text { F1 offglide } \\
\text { (Hz) }\end{array}$ & $\begin{array}{c}\text { F2 offglide } \\
(\mathrm{Hz})\end{array}$ \\
\hline oqm & 9 & 559 & 1028 & 548 & 1594 \\
\hline oqw & 16 & 553 & 993 & 570 & 1556 \\
\hline osm & 18 & 558 & 964 & 538 & 1633 \\
\hline osw & 10 & 477 & 923 & 464 & 1725 \\
\hline otw & 20 & 523 & 977 & 515 & 1770 \\
\hline yqm & 24 & 509 & 1032 & 510 & 1626 \\
\hline yqw & 9 & 505 & 952 & 515 & 1772 \\
\hline ysm & 11 & 542 & 1033 & 549 & 1701 \\
\hline ysw & 18 & 519 & 960 & 482 & 1670 \\
\hline ytm & 19 & 564 & 1077 & 541 & 1438 \\
\hline ytw & 16 & 493 & 693 & 498 & 1866 \\
\hline
\end{tabular}

\begin{tabular}{lcccc}
\hline DRESS & \multicolumn{4}{c}{ (ED wrt FLEECE) } \\
\hline Group & N & F1 (Hz) & F2 (Hz) & ED \\
\hline oqm & 241 & 511 & 2154 & 308 \\
oqw & 204 & 522 & 2113 & 308 \\
osm & 219 & 496 & 2182 & 233 \\
osw & 240 & 507 & 2183 & 255 \\
otw & 204 & 489 & 2232 & 212 \\
yqm & 227 & 492 & 2214 & 270 \\
yqw & 186 & 512 & 2158 & 304 \\
ysm & 184 & 523 & 2128 & 289 \\
ysw & 371 & 559 & 2065 & 377 \\
ytm & 293 & 500 & 2214 & 217 \\
ytw & 121 & 509 & 2200 & 244 \\
\hline
\end{tabular}




\begin{tabular}{lcccccc}
\hline FACE & \multicolumn{7}{c}{ (ED wrt FLEECE) } & & & & \\
\hline Group & $\mathbf{N}$ & $\begin{array}{c}\text { F1 onset } \\
(\mathbf{H z})\end{array}$ & $\begin{array}{c}\text { F2 onset } \\
\mathbf{( H z )}\end{array}$ & $\begin{array}{c}\text { F1 offglide } \\
\mathbf{( H z )}\end{array}$ & $\begin{array}{c}\text { F2 offglide } \\
(\mathbf{H z})\end{array}$ & $\begin{array}{c}\text { ED } \\
\text { (onset) }\end{array}$ \\
\hline oqm & 285 & 696 & 1764 & 568 & 1962 & 578 \\
oqw & 232 & 698 & 1809 & 563 & 1979 & 516 \\
osm & 427 & 746 & 1723 & 561 & 1990 & 586 \\
osw & 350 & 711 & 1791 & 540 & 2037 & 619 \\
otw & 263 & 710 & 1760 & 567 & 1939 & 571 \\
yqm & 359 & 664 & 1857 & 548 & 1980 & 507 \\
yqw & 239 & 686 & 1894 & 509 & 2096 & 549 \\
ysm & 342 & 669 & 1875 & 527 & 2088 & 492 \\
ysw & 463 & 647 & 1903 & 515 & 2087 & 527 \\
ytm & 357 & 682 & 1913 & 534 & 2094 & 612 \\
ytw & 178 & 628 & 1954 & 501 & 2087 & 433 \\
\hline
\end{tabular}

\begin{tabular}{|c|c|c|c|}
\hline \multicolumn{4}{|c|}{ FLEECE } \\
\hline Group & $\mathbf{N}$ & F1 (Hz) & F2 (Hz) \\
\hline oqm & 367 & 459 & 2363 \\
\hline oqw & 359 & 463 & 2305 \\
\hline osm & 431 & 429 & 2374 \\
\hline osw & 451 & 446 & 2342 \\
\hline otw & 281 & 456 & 2364 \\
\hline yqm & 422 & 442 & 2368 \\
\hline yqw & 340 & 445 & 2320 \\
\hline ysm & 414 & 428 & 2373 \\
\hline ysw & 507 & 447 & 2363 \\
\hline ytm & 473 & 435 & 2348 \\
\hline ytw & 228 & 428 & 2393 \\
\hline
\end{tabular}

\begin{tabular}{lccc}
\hline FOOT & & & \\
\hline Group & N & F1 (Hz) & F2 (Hz) \\
\hline oqm & 30 & 530 & 1301 \\
oqw & 29 & 525 & 1369 \\
osm & 58 & 515 & 1349 \\
osw & 63 & 530 & 1169 \\
otw & 35 & 518 & 1232 \\
yqm & 41 & 511 & 1371 \\
yqw & 42 & 516 & 1358 \\
ysm & 28 & 504 & 1297 \\
ysw & 42 & 520 & 1504 \\
ytm & 43 & 523 & 1295 \\
ytw & 25 & 537 & 1091 \\
\hline
\end{tabular}




\section{GOAT}

\begin{tabular}{lccccc}
\hline Group & N & $\begin{array}{c}\text { F1 onset } \\
(\mathbf{H z})\end{array}$ & $\begin{array}{c}\text { F2 onset } \\
\mathbf{( H z )}\end{array}$ & $\begin{array}{c}\text { F1 offglide } \\
(\mathbf{H z})\end{array}$ & $\begin{array}{c}\text { F2 offglide } \\
\mathbf{( H z )}\end{array}$ \\
\hline oqm & 194 & 662 & 1511 & 595 & 1599 \\
oqw & 171 & 682 & 1536 & 606 & 1564 \\
osm & 285 & 697 & 1538 & 618 & 1599 \\
osw & 244 & 668 & 1467 & 590 & 1540 \\
otw & 152 & 662 & 1527 & 587 & 1564 \\
yqm & 279 & 625 & 1491 & 571 & 1521 \\
yqw & 190 & 626 & 1428 & 578 & 1443 \\
ysm & 228 & 631 & 1499 & 569 & 1560 \\
ysw & 301 & 617 & 1438 & 573 & 1416 \\
ytm & 308 & 636 & 1463 & 569 & 1477 \\
ytw & 132 & 636 & 1531 & 579 & 1584 \\
\hline
\end{tabular}

\section{GOOSE}

\begin{tabular}{lccc}
\hline Group & N & F1 (Hz) & F2 (Hz) \\
\hline oqm & 229 & 461 & 1886 \\
oqw & 167 & 469 & 1866 \\
osm & 268 & 459 & 1887 \\
osw & 285 & 449 & 1908 \\
otw & 181 & 454 & 1842 \\
yqm & 229 & 439 & 1857 \\
yqw & 170 & 443 & 1817 \\
ysm & 205 & 424 & 1860 \\
ysw & 253 & 445 & 1930 \\
ytm & 291 & 435 & 1833 \\
ytw & 112 & 444 & 1933 \\
\hline
\end{tabular}

\begin{tabular}{lcccc}
\hline KIT & \multicolumn{6}{c}{ (ED wrt FLEECE) } & & \\
\hline Group & N & F1 (Hz) & F2 (Hz) & ED \\
\hline oqm & 142 & 565 & 1865 & 527 \\
oqw & 169 & 576 & 1807 & 571 \\
osm & 121 & 537 & 1901 & 468 \\
osw & 212 & 549 & 1880 & 513 \\
otw & 107 & 546 & 1942 & 446 \\
yqm & 234 & 530 & 1944 & 436 \\
yqw & 149 & 549 & 1871 & 504 \\
ysm & 121 & 539 & 1888 & 487 \\
ysw & 315 & 534 & 1843 & 527 \\
ytm & 195 & 571 & 1830 & 552 \\
ytw & 73 & 554 & 1895 & 483 \\
\hline
\end{tabular}




\begin{tabular}{lccc}
\hline LOT & & & \\
\hline Group & N & F1 (Hz) & F2 (Hz) \\
\hline oqm & 233 & 661 & 1181 \\
oqw & 244 & 665 & 1139 \\
osm & 324 & 699 & 1196 \\
osw & 309 & 661 & 1155 \\
otw & 210 & 667 & 1134 \\
yqm & 340 & 652 & 1158 \\
yqw & 245 & 668 & 1146 \\
ysm & 255 & 683 & 1123 \\
ysw & 361 & 686 & 1144 \\
ytm & 319 & 663 & 1184 \\
ytw & 136 & 691 & 1204 \\
\hline
\end{tabular}

\begin{tabular}{lcccccc}
\hline MOUTH & \multicolumn{7}{c}{ (ED wrt START) } \\
\hline Group & $\mathbf{N}$ & $\begin{array}{c}\text { F1 onset } \\
(\mathbf{H z})\end{array}$ & $\begin{array}{c}\text { F2 onset } \\
\mathbf{( H z )}\end{array}$ & $\begin{array}{c}\text { F1 offglide } \\
\mathbf{( H z )}\end{array}$ & $\begin{array}{c}\text { F2 offglide } \\
\mathbf{( H z )}\end{array}$ & $\begin{array}{c}\text { ED } \\
\text { (onset) }\end{array}$ \\
\hline oqm & 154 & 733 & 1744 & 766 & 1488 & 315 \\
oqw & 109 & 707 & 1709 & 736 & 1503 & 302 \\
osm & 161 & 686 & 1852 & 717 & 1553 & 418 \\
osw & 173 & 738 & 1648 & 719 & 1381 & 257 \\
otw & 124 & 704 & 1748 & 720 & 1462 & 329 \\
yqm & 168 & 724 & 1710 & 711 & 1458 & 299 \\
yqw & 117 & 712 & 1639 & 695 & 1391 & 268 \\
ysm & 186 & 716 & 1695 & 711 & 1398 & 305 \\
ysw & 214 & 735 & 1674 & 721 & 1364 & 290 \\
ytm & 201 & 729 & 1729 & 714 & 1405 & 335 \\
ytw & 100 & 754 & 1572 & 675 & 1315 & 229 \\
\hline
\end{tabular}

\begin{tabular}{lccccc}
\hline NEAR & \multicolumn{5}{c}{} \\
\hline Group & N & $\begin{array}{c}\text { F1 onset } \\
(\mathbf{H z})\end{array}$ & $\begin{array}{c}\text { F2 onset } \\
\mathbf{( H z )}\end{array}$ & $\begin{array}{c}\text { F1 offglide } \\
\mathbf{( H z )}\end{array}$ & $\begin{array}{c}\text { F2 offglide } \\
\text { (Hz) }\end{array}$ \\
\hline oqm & 24 & 462 & 2367 & 519 & 2136 \\
oqw & 35 & 474 & 2340 & 523 & 2113 \\
osm & 27 & 461 & 2397 & 546 & 2113 \\
osw & 46 & 452 & 2397 & 524 & 2085 \\
otw & 23 & 446 & 2266 & 515 & 2006 \\
yqm & 42 & 450 & 2453 & 545 & 2161 \\
yqw & 29 & 454 & 2317 & 547 & 1951 \\
ysm & 54 & 486 & 2320 & 546 & 2062 \\
ysw & 56 & 467 & 2313 & 580 & 1950 \\
ytm & 32 & 458 & 2325 & 567 & 2023 \\
ytw & 6 & 476 & 2284 & 634 & 1875 \\
\hline
\end{tabular}




\begin{tabular}{lccc}
\hline NORTH & & & \\
\hline Group & N & F1 (Hz) & F2 (Hz) \\
\hline oqm & 101 & 509 & 950 \\
oqw & 58 & 507 & 888 \\
osm & 123 & 485 & 891 \\
osw & 104 & 477 & 947 \\
otw & 73 & 493 & 1005 \\
yqm & 88 & 509 & 894 \\
yqw & 57 & 486 & 820 \\
ysm & 67 & 530 & 880 \\
ysw & 113 & 502 & 829 \\
ytm & 124 & 489 & 943 \\
ytw & 60 & 491 & 859 \\
\hline
\end{tabular}

\begin{tabular}{llccc}
\hline NURSE & \multicolumn{4}{c}{ (ED wrt GOOSE) } \\
\hline Group & N & F1 (Hz) & F2 (Hz) & ED \\
\hline oqm & 75 & 509 & 1777 & 193 \\
oqw & 56 & 506 & 1774 & 252 \\
osm & 88 & 480 & 1877 & 140 \\
osw & 79 & 483 & 1730 & 237 \\
otw & 65 & 468 & 1837 & 106 \\
yqm & 50 & 514 & 1769 & 176 \\
yqw & 62 & 481 & 1831 & 131 \\
ysm & 61 & 559 & 1635 & 305 \\
ysw & 58 & 514 & 1773 & 207 \\
ytm & 94 & 553 & 1693 & 258 \\
ytw & 70 & 577 & 1585 & 331 \\
\hline
\end{tabular}

\section{PRICE}

\begin{tabular}{lccccc}
\hline Group & N & $\begin{array}{c}\text { F1 onset } \\
(\mathbf{H z})\end{array}$ & $\begin{array}{c}\text { F2 onset } \\
(\mathbf{H z})\end{array}$ & $\begin{array}{c}\text { F1 offglide } \\
(\mathbf{H z})\end{array}$ & $\begin{array}{c}\text { F2 offglide } \\
(\mathbf{H z})\end{array}$ \\
\hline oqm & 327 & 714 & 1393 & 729 & 1540 \\
oqw & 291 & 711 & 1370 & 706 & 1530 \\
osm & 346 & 713 & 1346 & 705 & 1527 \\
osw & 420 & 706 & 1380 & 701 & 1570 \\
otw & 255 & 707 & 1342 & 692 & 1573 \\
yqm & 457 & 679 & 1424 & 690 & 1635 \\
yqw & 321 & 693 & 1445 & 641 & 1756 \\
ysm & 392 & 710 & 1407 & 687 & 1647 \\
ysw & 462 & 695 & 1428 & 653 & 1667 \\
ytm & 443 & 676 & 1400 & 680 & 1588 \\
ytw & 179 & 775 & 1455 & 638 & 1774 \\
\hline
\end{tabular}




\begin{tabular}{lccccc}
\hline SQUARE & \multicolumn{5}{c}{} \\
\hline Group & N & $\begin{array}{c}\text { F1 onset } \\
(\mathbf{H z})\end{array}$ & $\begin{array}{c}\text { F2 onset } \\
\mathbf{( H z )}\end{array}$ & $\begin{array}{c}\text { F1 offglide } \\
\mathbf{( H z )}\end{array}$ & $\begin{array}{c}\text { F2 offglide } \\
\mathbf{( H z )}\end{array}$ \\
\hline oqm & 47 & 526 & 2081 & 550 & 1966 \\
oqw & 59 & 531 & 2158 & 561 & 1975 \\
osm & 70 & 487 & 2178 & 523 & 2071 \\
osw & 64 & 512 & 2116 & 550 & 1958 \\
otw & 67 & 484 & 2158 & 523 & 2005 \\
yqm & 82 & 491 & 2007 & 554 & 1850 \\
yqw & 64 & 504 & 2106 & 580 & 1888 \\
ysm & 63 & 535 & 1969 & 569 & 1864 \\
ysw & 84 & 518 & 2039 & 562 & 1849 \\
ytm & 103 & 534 & 2123 & 578 & 1960 \\
ytw & 42 & 497 & 2175 & 592 & 1927 \\
\hline
\end{tabular}

\begin{tabular}{lccc}
\hline START & & & \\
\hline Group & N & F1 (Hz) & F2 (Hz) \\
\hline oqm & 124 & 843 & 1517 \\
oqw & 60 & 839 & 1483 \\
osm & 150 & 852 & 1540 \\
osw & 100 & 820 & 1466 \\
otw & 51 & 833 & 1526 \\
yqm & 98 & 840 & 1449 \\
yqw & 74 & 832 & 1437 \\
ysm & 123 & 849 & 1446 \\
ysw & 111 & 844 & 1421 \\
ytm & 90 & 843 & 1426 \\
ytw & 50 & 835 & 1470 \\
\hline
\end{tabular}

\begin{tabular}{lccc}
\hline STRUT & & & \\
\hline Group & N & F1 (Hz) & F2 (Hz) \\
\hline oqm & 336 & 750 & 1479 \\
oqw & 270 & 751 & 1435 \\
osm & 335 & 779 & 1500 \\
osw & 342 & 768 & 1452 \\
otw & 244 & 737 & 1467 \\
yqm & 391 & 716 & 1430 \\
yqw & 293 & 748 & 1464 \\
ysm & 314 & 740 & 1429 \\
ysw & 404 & 737 & 1433 \\
ytm & 432 & 722 & 1410 \\
ytw & 191 & 745 & 1489 \\
\hline
\end{tabular}




\begin{tabular}{cccc}
\hline \multicolumn{2}{l}{ THOUGHT } & & \\
\hline Group & N & F1 (Hz) & F2 (Hz) \\
\hline oqm & 44 & 526 & 1006 \\
oqw & 40 & 520 & 1018 \\
osm & 55 & 486 & 902 \\
osw & 62 & 481 & 1046 \\
otw & 35 & 483 & 957 \\
yqm & 43 & 520 & 938 \\
yqw & 40 & 516 & 963 \\
ysm & 41 & 537 & 931 \\
ysw & 91 & 569 & 928 \\
ytm & 65 & 519 & 1030 \\
ytw & 10 & 607 & 1089 \\
\hline
\end{tabular}

\begin{tabular}{lcccc}
\hline TRAP & \multicolumn{4}{c}{ (ED wrt START) } \\
\hline Group & N & F1 (Hz) & F2 (Hz) & ED \\
\hline oqm & 157 & 659 & 2022 & 578 \\
oqw & 127 & 683 & 1964 & 516 \\
osm & 200 & 671 & 2034 & 586 \\
osw & 251 & 684 & 2072 & 619 \\
otw & 159 & 667 & 2017 & 571 \\
yqm & 245 & 679 & 1953 & 507 \\
yqw & 153 & 680 & 1998 & 549 \\
ysm & 234 & 722 & 1950 & 492 \\
ysw & 245 & 673 & 1972 & 527 \\
ytm & 318 & 691 & 2066 & 612 \\
ytw & 93 & 722 & 1889 & 433 \\
\hline
\end{tabular}




\section{Appendix E}

Wellington 6012

New Zealand

\section{PARTICIPANT INFORMATION SHEET}

Project title: The practice of identity: gender and performativity in New Zealand and Canadian English Researcher: Evan Hazenberg

\section{Introduction}

My name is Evan Hazenberg, and I am a PhD student in the School of Linguistics and Applied Language Studies (LALS) at the Victoria University of Wellington. I'm studying the role that gender plays in the linguistic choices we make in everyday speech, both here in New Zealand and in Canada. I already have a collection of interviews from Canada, and I'm looking to create a similar one here in New Zealand. If you are a native speaker of New Zealand English, I would like to invite you to participate in my research.

\section{Procedures}

Participation is strictly voluntary. If you agree to participate, you will be committing to one digitallyrecorded (audio-only) conversation lasting between one and two hours. We can do the recording wherever is most convenient for you - your home, my home, somewhere on campus, somewhere else - so long as it's fairly quiet. The conversation will be in two parts: the first will be a casual chat about whatever you feel like talking about; the second (and generally shorter) part will be more like an interview, where l ask you a few questions about your impressions of gender and language. There are no right or wrong answers - I want to know what you think is going on.

Your confidentiality is important to me, so your recording will be assigned a number and a pseudonym, and these are the only identifiers that will be used in referring to the recording. I will be transcribing the interviews, and then anonymizing both the recording and the transcript - that is, I'll take out anything that might identify you as the speaker. I'll keep one unaltered copy of the recording as a backup, but I will be the only person with access to it, and the research itself will be conducted using the anonymized versions.

If you agree to participate and then later change your mind, that's okay - you're free to withdraw from the project at any time up to one year from the date of recording. After that point, it may be difficult to remove your data from any analyses that have already been carried out, but you will always be welcome to indicate that you do you not want your data used in future projects. Similarly, you're also free to stipulate that certain portions of the recording be either deleted, or not used in subsequent analysis.

\section{Use of the data}

Your recording and transcript will be used in both qualitative and quantitative research around gender and language use. It will primarily be used for my doctoral thesis, and any excerpts that find their way into either the written thesis or any associated presentations will be anonymized, and attributed only to your pseudonym. I am the primary analyst for this project, but my supervisor and co-supervisor will also have access to the recordings and the transcripts.

Because I'm building a collection of sociolinguistic data, your recording may be used in more than one project. This means that your recording may be shared with co-investigators in future research projects; if this is the case, these researchers will only have access to the anonymized versions of the recording and transcript, and they will only know your pseudonym and some fairly basic demographic information about you (such as your age, your gender, and where you grew up). If you prefer, you also have the option of stipulating that I am the only researcher who can access your recording or 
transcript at all. What this means is, unless you explicitly state otherwise, your recording and transcript will be kept indefinitely as part of a sociolinguistic data set, with all confidentiality precautions maintained for the lifetime of the archive. It will be stored as digital files on an external hard drive, which will be stored securely in a locked cabinet at all times. Note that, as new storage media are developed, the files may be changed to a new format, but will still be kept secure.

If you would like to have a copy of your recording or your transcript, I am happy to provide them to you. Likewise, if you would like to be kept up to date with either my doctoral thesis or any other publications that arise from use of your data, I am happy to share these with you. Any address or contact information that you provide me will be used for the sole purpose of transmitting information to you, and will not be shared with anyone else.

\section{Contact details}

If you have any questions, comments, concerns or thoughts about the project as a whole, please don't hesitate to get in touch with me:

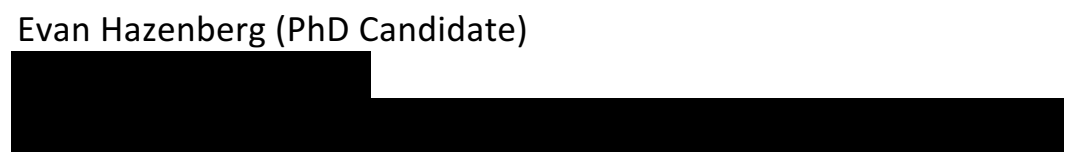

If you have any questions or concerns relating specifically to the collection or use of data as described in this document, you are invited to direct your inquiries to any of the following:
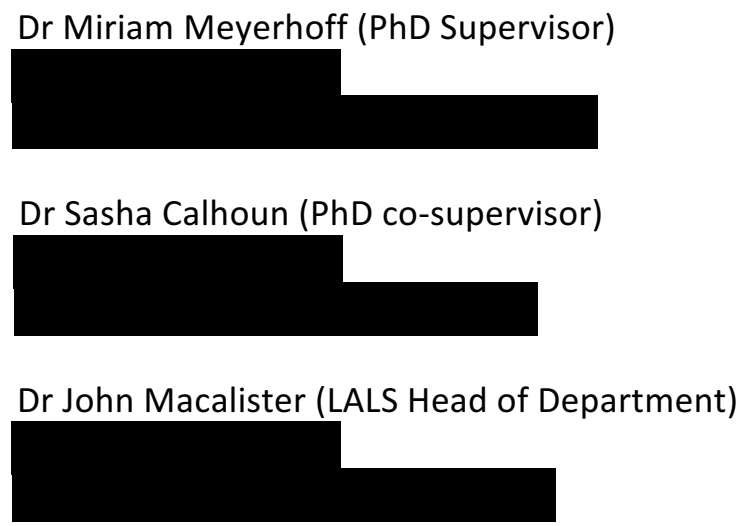

For queries regarding ethical concerns, you may contact:

Dr Allison Kirkman (Convener, VUW Human Ethics Committee)

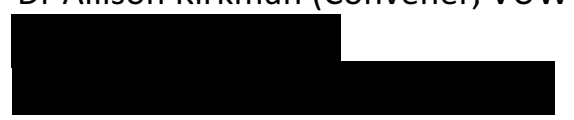




\section{CONSENT FORM}

THIS FORM WILL BE HELD FOR A PERIOD OF 6 YEARS

Project title: The practice of identity: gender and performativity in New Zealand and Canadian English Researcher: Evan Hazenberg

I have read the Participant Information sheet and I understand the intent and nature of the research project. I understand that my participation is strictly voluntary. I have had the opportunity to ask and receive satisfactory questions about the research. I understand that I may choose to withdraw from the study at any time within one year of this date, and to change the conditions under which my recording and transcript are used for future projects at any time; otherwise, I understand that the data will be stored in a secure location indefinitely.

Please read the following, and check the boxes that you agree to. You may choose to check only some of the boxes and not others, or you may check all of them. Your check mark(s) plus your signature at the end of the form indicate that you consent to that component of the study.

$\square$ I agree to participate in this interview, either individually or as part of a small group (please cross out whichever does not apply).

$\square \quad$ I am aware that our conversation is being digitally recorded, and that I have the right to request erasure of any portion of the recording that I am uncomfortable with, or to withdraw from the study at any time.

$\square \quad$ I grant you permission to use the interview material for any academic purposes relating to your doctoral research, such as discussions, presentations, teaching and any published or unpublished works. I understand that my confidentiality will be maintained at all times.

$\square \quad$ I understand that I have one (1) year from this date to withdraw my participation from the doctoral research project.

$\square \quad$ I grant you permission to keep and use my recording and transcript as part of a larger collection of sociolinguistic data, with the understanding that my confidentiality will be maintained at all times.

$\square \quad$ I understand that I may, at any time, withdraw my participation from any future project not already underway.

$\square \quad$ I grant you permission to share anonymized versions of my recording and transcript with other co-investigators in future collaborative linguistic studies. 


\section{REQUEST FOR FURTHER CONTACT}

I would like to have the following information or documents sent to me:

$\square$ a digital copy of my recording

$\square \quad$ a transcript of my recording

$\square$ summaries of any research findings that stem from this project

$\square$ electronic copies of any journal papers and/or presentations that stem from this project

I would like the above to be sent to me at the following address(es):

name (for use in

correspondence)

email address

postal address 